\title{
Degenerations of Kählerian K3 surfaces with finite symplectic automorphism groups
}

\author{
Viacheslav V. Nikulin
}

\begin{abstract}
Using results of our preprint "Kählerian K3 surfaces and Niemeier lattices" arXiv:1109.2879 (and the corresponding papers), we classify degenerations of Kählerian K3 surfaces with finite symplectic automorphism groups.

Dedicated to the memory of Andrey Todorov
\end{abstract}

\section{Introduction}

Using results of our preprint "Kählerian K3 surfaces and Niemeier lattices" arXiv:1109.2879 [17] (and the corresponding papers), we classify degenerations of Kählerian K3 surfaces with finite symplectic automorphism groups.

Here we mainly consider degenerations of codimension 1. We describe possible Dynkin diagrams and discriminant groups of the degenerations. See Section 6. In Section 5, one can find detailed description of possible degenerations using markings by Niemeier lattices; it uses classification of so called KahK3 conjugacy classes of automorphism groups of Niemeier lattices obtained in [17], [18] and Section 4 here. In Section 7 we formulate an important Conjecture.

In Section 3, we consider an example of Kummer surfaces which can be considered as a degeneration of codimension 1 of Kählerian K3 surfaces with symplectic automorphism group $\left(C_{2}\right)^{4}$, and the general theory.

In Section 2, we remind to a reader results of [16] about existence of a primitive embedding of a lattice (i.e., an integral symmetric bilinear form) into one of even unimodular lattices of the fixed signature. See Theorem 1. It is crucial for our classification since by the epimorphicity of Torelli map for Kählerian K3 surfaces proved by A. Todorov [21] and Y. Siu [20], it permits to describe possible Picard lattices of K3 surfaces. All the time we use Programs 5 and 6 from Section 8 , Appendix based on Theorem 1 . 
We hope to give more exact results (e.g. see Conjecture 4) and consider other cases (for example, degenerations of other codimensions) in further publications.

\section{Existence of a primitive embedding of an even lattice into even unimodular lattices, according to [16]}

In this paper, we use notations, definitions and results of [16] about lattices (that is nondegenerate integral (over $\mathbb{Z}$ ) symmetric bilinear forms). In particular, $\oplus$ denotes the orthogonal sum of lattices, quadratic forms. For a prime $p$, we denote by $\mathbb{Z}_{p}$ the ring of $p$-adic integers, and by $\mathbb{Z}_{p}^{*}$ its group of invertible elements.

Let $S$ be a lattice. Let $A_{S}=S^{*} / S$ be its discriminant group, and $q_{S}$ its discriminant quadratic form on $A_{S}$ where we assume that the lattice $S$ is even: that is $x^{2}$ is even for any $x \in S$. We denote by $l\left(A_{S}\right)$ the minimal number of generators of the finite Abelian group $A_{S}$, and by $\left|A_{S}\right|$ its order. For a prime $p$, we denote by $q_{S p}=q_{S \otimes \mathbb{Z}_{p}}$ the $p$-component of $q_{S}$ (equivalently, the discriminant quadratic form of the $p$-adic lattice $S \otimes \mathbb{Z}_{p}$ ). A quadratic form on a group of order 2 is denoted by $q_{\theta}^{(2)}(2)$. A $p$-adic lattice $K\left(q_{S_{p}}\right)$ or the rank $l\left(A_{S_{p}}\right)$ with the discriminant quadratic form $q_{S_{p}}$ is denoted by $K\left(q_{S_{p}}\right)$. It is unique, up to isomorphisms, for $p \neq 2$, and for $p=2$, if $q_{S_{2}} \neq q_{\theta}^{(2)}(2) \oplus q^{\prime}$. We have the following result where an embedding $S \subset L$ of lattices is called primitive if $L / S$ has no torsion.

Theorem 1. (Theorem 1.12.2 in [16]).

Let $S$ be an even lattice of the signature $\left(t_{(+)}, t_{(-)}\right)$, and $l_{(+)}, l_{(-)}$are integers.

Then, there exists a primitive embedding of $S$ into one of even unimodular lattices of the signature $\left(l_{(+)}, l_{(-)}\right)$if and only if the following conditions satisfy:

(1) $l_{(+)}-l_{(-)} \equiv 0 \bmod 8$;

(2) $l_{(+)}-t_{(+)} \geq 0, l_{(-)}-t_{(-)} \geq 0, l_{(+)}+l_{(-)}-t_{(+)}-t_{(-)} \geq l\left(A_{S}\right)$;

(3) $(-1)^{l_{(+)}-t_{(+)}}\left|A_{S}\right| \equiv \operatorname{det} K\left(q_{S_{p}}\right) \bmod \left(\mathbb{Z}_{p}{ }^{*}\right)^{2}$ for each odd prime $p$ such that $l_{(+)}+l_{(-)}-$ $t_{(+)}-t_{(-)}=l\left(A_{S_{p}}\right)$;

(4) $\left|A_{S}\right| \equiv \pm \operatorname{det} K\left(q_{S_{2}}\right) \bmod \left(\mathbb{Z}_{2}{ }^{*}\right)^{2}$, if $l_{(+)}+l_{(-)}-t_{(+)}-t_{(-)}=l\left(A_{S_{2}}\right)$ and $q_{S_{2}} \neq$ $q_{\theta}^{(2)}(2) \oplus q^{\prime}$

Remark that if the last inequality in (2) is strict then one does not need the conditions (3) and (4). If $q_{S_{2}} \cong q_{\theta}^{(2)}(2) \oplus q^{\prime}$, then one does not need the condition (4). 


\section{An example related to Kummer surfaces, and the general theory.}

Let us consider Kählerian K3 surfaces which are Kummer sufaces. They are minimal desingularizations of the quotients of 2-dimensional complex tori by the involution of the inversion. A general Kummer surface $X$ has the Picard number 16 and has 16 non-singular rational curves which define the Dynkin diagram $16 \mathbb{A}_{1}$. Moreover, $X$ has the symplectic automorphism group $\left(C_{2}\right)^{4}$ defined by translations of order two elements of the tori. General Kählerian K3 surfaces with symplectic automorphism group $\left(C_{2}\right)^{4}$ have Picard number 15 . See [13], [15]. Thus Kummer surfaces can be considered as the degeneration of codimension 1 of Kählerian K3 surfaces with symplectic automorphism group $\left(C_{2}\right)^{4}$.

This classical example raises the following interesting questions.

Do Kählerian K3 surfaces with finite symplectic automorphism group $\left(C_{2}\right)^{4}$ have other degenerations of codimension 1 which are different from Kummer?

What are degenerations of codimension 1 (and other codimensions) of K3 surfaces with other finite symplectic automorphism groups?

Using results of our preprint [17], we can answer this questions.

For example, we show that Kählerian K3 surfaces with finite symplectic automorphism group $\left(C_{2}\right)^{4}$ have another degeneration of codimension 1 which has the type $4 \mathbb{A}_{1}$. It follows from Theorem 3 below. Thus, the Picard lattice of the K3 surface has the rank 16, the K3 surface has exactly 4 non-singular rational curves which have the Dynkin diagram $4 \mathbb{A}_{1}$, and it has the automorphism group $\left(C_{2}\right)^{4}$. By [3], [7] and [12], it can be considered as the minimal resolution of singularities of the complex surface with the corresponding four Du Val singularities of the type $\mathbb{A}_{1}$ and the automorphism group $\left(C_{2}\right)^{4}$.

Using markings of Kählerian K3 surfaces by Niemeier lattices which was developed in [17], this is reduced to the following question. We use notations and results from [17].

Let $N_{j}$ be one of 23 Niemeier lattices which have non-trivial root systems. We fix the bases $P\left(N_{j}\right)$ of the root system and consider the automorphism group $A\left(N_{j}\right)$ of $N_{j}$ which preserves the bases $P\left(N_{j}\right)$. Let $H \subset A\left(N_{j}\right)$ be a subgroup of $A\left(N_{j}\right)$. We denote by $\left(N_{j}\right)_{H}=$ $\left(N_{j}^{H}\right)_{N_{j}}^{\perp} \subset N_{j}$ the coinvariant sublattice of $H$. We say that $H$ is maximal if $H=C l o s(H)$ is the maximal subgroup of $A\left(N_{j}\right)$ with the same coinvariant sublattice $N_{H}$. We can restrict considering the maximal case only. A subgroup $H$ is Kählerian K3 automorphism group if $N_{H}$ has a primitive embedding into the lattice $L_{K 3}$ which is an even unimodular lattice of the signature $(3,19)$. It is isomorphic to the cohomology lattice $H^{2}(X, \mathbb{Z})$ of Kählerian $\mathrm{K} 3$ surfaces. Equivalently, $N_{H}$ satisfies conditions of Theorem 1 for $l_{(+)}=3, l_{(-)}=19$.

Let $\alpha \in P\left(N_{j}\right)$ and $H(\alpha)$ is the orbit of $H$ for $\alpha \in P\left(N_{j}\right)$. The primitive sublattice $S=\left[N_{H}, \alpha\right]_{p r} \subset N_{j}$ contains $H(\alpha)$ and $H(\alpha)$ gives the basis of the system of roots with 
square $(-2)$ in $S$. If $S$ has a primitive embedding into $L_{K 3}$ (equivalently, if $S$ satisfies Theorem 1 for $\left.l_{(+)}=3, l_{(-)}=19\right)$, then $S$ can be considered as Picard lattice of general Kählerian K3 surfaces with symplectic automorphism group $H$ and classes of non-singular rational curves $H(\alpha)$. Here we use epimorphicity of the period map for Kählerian K3 surfaces, see A. Todorov [21] and Y. Siu [20] and global Torelli Theorem for K3 surfaces [4] (see also [10] and [19]). They permit to describe possible Picard lattices of Since rk $S=\operatorname{rk} N_{H}+1$, these K3 surfaces can be considered as degeneration of codimension 1 of Kählerian K3 surfaces with the automorphism group $H$. The primitive embedding $S \subset N_{j}$ can be considered as marking of the Kählerian K3 surfaces by the Niemeier lattice $N_{j}$. See [17] for details.

In [17] we described Kählerian K3 conjugacy classes for Niemeier lattices $N_{j}, j=$ $1, \ldots, 23$. Thus, using these classification and Theorem 1, we can describe degenerations of codimension 1 of Kählerian K3 surfaces with finite symplectic automorphism groups. By considering several orbits of $H$, we can describe all degenerations.

For example, the group $\left(C_{2}\right)^{4}$ corresponds to the following Kählerian K3 conjugacy classes for $N_{23}=N\left(24 A_{1}\right)$. This case can be marked by $N_{23}$ only. See [17]. We use the basis $\alpha_{1}, \ldots, \alpha_{24}$ with the Dynkin diagram $24 \mathbb{A}_{1}$ for the roots system $24 A_{1}$ of $N_{23}$.

$\mathbf{n}=\mathbf{2 1}, H \cong C_{2}^{4}(|H|=16, i=14): \operatorname{rk} N_{H}=15,\left(N_{H}\right)^{*} / N_{H} \cong \mathbb{Z} / 8 \mathbb{Z} \times(\mathbb{Z} / 2 \mathbb{Z})^{6}$.

$$
\begin{gathered}
H_{21,1}=\left[\left(\alpha_{2} \alpha_{20}\right)\left(\alpha_{3} \alpha_{10}\right)\left(\alpha_{5} \alpha_{6}\right)\left(\alpha_{8} \alpha_{11}\right)\left(\alpha_{9} \alpha_{21}\right)\left(\alpha_{12} \alpha_{22}\right)\left(\alpha_{17} \alpha_{23}\right)\left(\alpha_{19} \alpha_{24}\right),\right. \\
\left(\alpha_{2} \alpha_{19}\right)\left(\alpha_{3} \alpha_{5}\right)\left(\alpha_{6} \alpha_{10}\right)\left(\alpha_{8} \alpha_{9}\right)\left(\alpha_{11} \alpha_{21}\right)\left(\alpha_{12} \alpha_{23}\right)\left(\alpha_{17} \alpha_{22}\right)\left(\alpha_{20} \alpha_{24}\right), \\
\left(\alpha_{1} \alpha_{16}\right)\left(\alpha_{2} \alpha_{20}\right)\left(\alpha_{3} \alpha_{6}\right)\left(\alpha_{5} \alpha_{10}\right)\left(\alpha_{12} \alpha_{23}\right)\left(\alpha_{14} \alpha_{18}\right)\left(\alpha_{17} \alpha_{22}\right)\left(\alpha_{19} \alpha_{24}\right), \\
\left.\left(\alpha_{1} \alpha_{14}\right)\left(\alpha_{2} \alpha_{24}\right)\left(\alpha_{3} \alpha_{5}\right)\left(\alpha_{6} \alpha_{10}\right)\left(\alpha_{12} \alpha_{22}\right)\left(\alpha_{16} \alpha_{18}\right)\left(\alpha_{17} \alpha_{23}\right)\left(\alpha_{19} \alpha_{20}\right)\right]
\end{gathered}
$$

with orbits $\left\{\alpha_{1}, \alpha_{16}, \alpha_{14}, \alpha_{18}\right\},\left\{\alpha_{2}, \alpha_{20}, \alpha_{19}, \alpha_{24}\right\},\left\{\alpha_{3}, \alpha_{10}, \alpha_{5}, \alpha_{6}\right\},\left\{\alpha_{8}, \alpha_{11}, \alpha_{9}, \alpha_{21}\right\}$, $\left\{\alpha_{12}, \alpha_{22}, \alpha_{23}, \alpha_{17}\right\}$

$$
\begin{gathered}
H_{21,2}=\left[\left(\alpha_{1} \alpha_{3}\right)\left(\alpha_{2} \alpha_{23}\right)\left(\alpha_{5} \alpha_{14}\right)\left(\alpha_{6} \alpha_{16}\right)\left(\alpha_{10} \alpha_{18}\right)\left(\alpha_{12} \alpha_{20}\right)\left(\alpha_{17} \alpha_{24}\right)\left(\alpha_{19} \alpha_{22}\right),\right. \\
\left(\alpha_{1} \alpha_{2}\right)\left(\alpha_{3} \alpha_{23}\right)\left(\alpha_{5} \alpha_{17}\right)\left(\alpha_{6} \alpha_{12}\right)\left(\alpha_{10} \alpha_{22}\right)\left(\alpha_{14} \alpha_{24}\right)\left(\alpha_{16} \alpha_{20}\right)\left(\alpha_{18} \alpha_{19}\right), \\
\left(\alpha_{1} \alpha_{16}\right)\left(\alpha_{2} \alpha_{20}\right)\left(\alpha_{3} \alpha_{6}\right)\left(\alpha_{5} \alpha_{10}\right)\left(\alpha_{12} \alpha_{23}\right)\left(\alpha_{14} \alpha_{18}\right)\left(\alpha_{17} \alpha_{22}\right)\left(\alpha_{19} \alpha_{24}\right), \\
\left.\left(\alpha_{1} \alpha_{14}\right)\left(\alpha_{2} \alpha_{24}\right)\left(\alpha_{3} \alpha_{5}\right)\left(\alpha_{6} \alpha_{10}\right)\left(\alpha_{12} \alpha_{22}\right)\left(\alpha_{16} \alpha_{18}\right)\left(\alpha_{17} \alpha_{23}\right)\left(\alpha_{19} \alpha_{20}\right)\right]
\end{gathered}
$$

with orbits $\left\{\alpha_{1}, \alpha_{3}, \alpha_{2}, \alpha_{16}, \alpha_{14}, \alpha_{23}, \alpha_{6}, \alpha_{5}, \alpha_{20}, \alpha_{24}, \alpha_{18}, \alpha_{12}, \alpha_{17}, \alpha_{10}, \alpha_{19}, \alpha_{22}\right\}$ (we give only orbits with more than one element).

The group $H_{21,1}$ and its orbits with 4 elements (there are also four orbits with 1 element) give degenerations of the type $4 \mathbb{A}_{1}$ (by Theorem 11, only for these orbits the lattice $S$ has a 


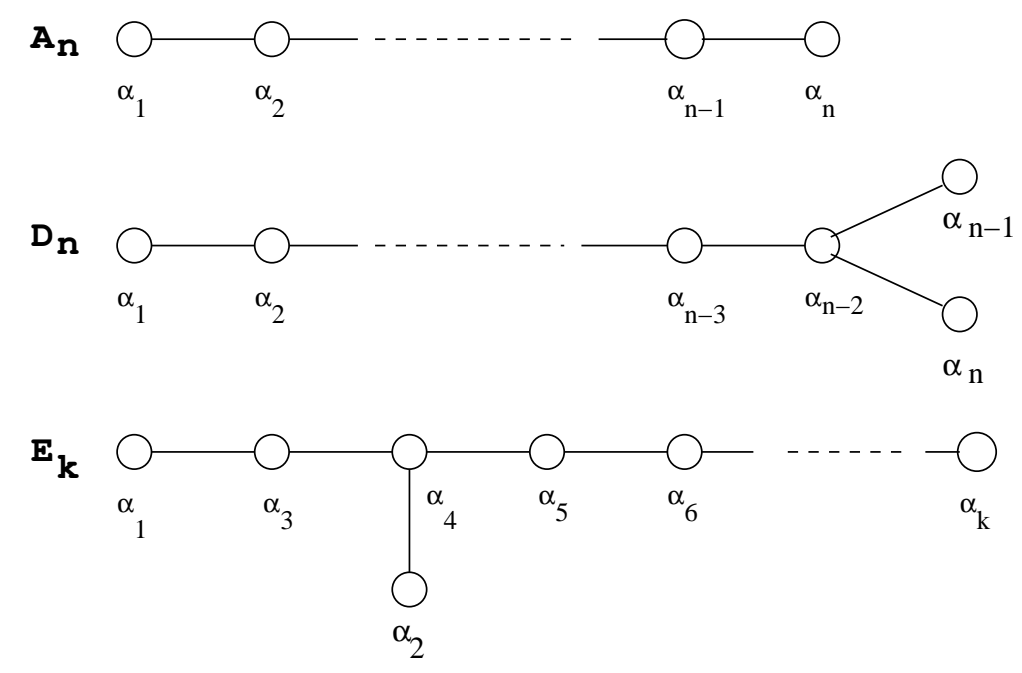

Figure 1: Bases of Dynkin diagrams $\mathbb{A}_{n}, \mathbb{D}_{n}, \mathbb{E}_{k}$.

primitive embedding into $L_{K 3}$ ). The group $H_{21,2}$ and its orbit with 16 elements gives the degeneration of the type $16 \mathbb{A}_{1}$ which gives the case of Kummer surfaces (by Theorem 1 , only for this orbit the lattice $S$ has a primitive embedding into $L_{K 3}$ ). Thus Kählerian K3 surfaces with symplectic automorphism group $H \cong\left(C_{2}\right)^{4}$ have codimension 1 degenerations only of the types $4 \mathbb{A}_{1}$ and $16 \mathbb{A}_{1}$. We also calculate the discriminant group $A_{S}=S^{*} / S$ of $S$ for both cases. See the case $n=21$ of Theorem 3 in Section 6 .

Similarly below we describe degenerations of codimension 1 which are marked by all Niemeier lattices. As a result, we obtain classification of types of degenerations of codimension 1 of Kählerian K3 surfaces. We hope to consider more exact classification and degenerations of arbitrary codimension in further variants of this paper and further publications.

\section{Classification of KahK3 conjugacy classes for $N_{j}, j=1, \ldots, 21$.}

We use the same notations as in [17] and [18].

Below, we use the basis of a root lattice $A_{n}, D_{n}$ or $E_{k}, k=6,7,8$, which is shown on Figure 1.

For $A_{n}, n \geq 1$, we denote $\varepsilon_{1}=\left(\alpha_{1}+2 \alpha_{2}+\cdots+n \alpha_{n}\right) /(n+1)$. It gives the generator of the discriminant group $A_{n}^{*} / A_{n} \cong \mathbb{Z} /(n+1) \mathbb{Z}$. 
For $D_{n}, n \geq 4$ and $n \equiv 0 \bmod 2$, we denote $\varepsilon_{1}=\left(\alpha_{1}+\alpha_{3}+\cdots+\alpha_{n-3}+\alpha_{n-1}\right) / 2$, $\varepsilon_{2}=\left(\alpha_{n-1}+\alpha_{n}\right) / 2, \varepsilon_{3}=\left(\alpha_{1}+\alpha_{3}+\cdots+\alpha_{n-3}+\alpha_{n}\right) / 2$. They give all non-zero elements of the discriminant group $D_{n}^{*} / D_{n} \cong(\mathbb{Z} / 2 \mathbb{Z})^{2}$.

For $D_{n}, n \geq 4$ and $n \equiv 1 \bmod 2$, we denote $\varepsilon_{1}=\left(\alpha_{1}+\alpha_{3}+\cdots+\alpha_{n-2}\right) / 2+\alpha_{n-1} / 4-\alpha_{n} / 4$, $\varepsilon_{2}=\left(\alpha_{n-1}+\alpha_{n}\right) / 2, \varepsilon_{3}=\left(\alpha_{1}+\alpha_{3}+\cdots+\alpha_{n-2}\right) / 2-\alpha_{n-1} / 4+\alpha_{n} / 4$. They give all non-zero elements of $D_{n}^{*} / D_{n} \cong \mathbb{Z} / 4 \mathbb{Z}$.

For $E_{6}$, we denote $\varepsilon_{1}=\left(\alpha_{1}-\alpha_{3}+\alpha_{5}-\alpha_{6}\right) / 3, \varepsilon_{2}=\left(-\alpha_{1}+\alpha_{3}-\alpha_{5}+\alpha_{6}\right) / 3$. They give all non-zero elements of $E_{6}^{*} / E_{6} \cong \mathbb{Z} / 3 \mathbb{Z}$.

For $E_{7}$, we denote $\varepsilon_{1}=\left(\alpha_{2}+\alpha_{5}+\alpha_{7}\right) / 2$. It gives the non-zero element of $E_{7}^{*} / E_{7} \cong \mathbb{Z} / 2 \mathbb{Z}$.

If the Dynkin diagram of a root lattice has several connected components, the second index of a basis numerates the corresponding connected component.

Case 21. For the Niemeier lattice

$$
\begin{gathered}
N=N_{21}=N\left(8 A_{3}\right)=\left[8 A_{3},[3(2001011)]\right]= \\
{\left[8 A_{3},-\varepsilon_{1,1}+2 \varepsilon_{1,2}+\varepsilon_{1,5}+\varepsilon_{1,7}+\varepsilon_{1,8},-\varepsilon_{1,1}+\varepsilon_{1,2}+2 \varepsilon_{1,3}+\varepsilon_{1,6}+\varepsilon_{1,8}\right.} \\
-\varepsilon_{1,1}+\varepsilon_{1,2}+\varepsilon_{1,3}+2 \varepsilon_{1,4}+\varepsilon_{1,7},-\varepsilon_{1,1}+\varepsilon_{1,3}+\varepsilon_{1,4}+2 \varepsilon_{1,5}+\varepsilon_{1,8} \\
-\varepsilon_{1,1}+\varepsilon_{1,2}+\varepsilon_{1,4}+\varepsilon_{1,5}+2 \varepsilon_{1,6},-\varepsilon_{1,1}+\varepsilon_{1,3}+\varepsilon_{1,5}+\varepsilon_{1,6}+2 \varepsilon_{1,7}, \\
\left.-\varepsilon_{1,1}+\varepsilon_{1,4}+\varepsilon_{1,6}+\varepsilon_{1,7}+2 \varepsilon_{1,8}\right]
\end{gathered}
$$

the group $A\left(N_{21}\right)$ has the order 2688 , and it is generated by

$$
\begin{gathered}
\widetilde{F 1}_{7}=\left(\alpha_{1,2} \alpha_{1,3} \alpha_{1,4} \alpha_{1,5} \alpha_{1,6} \alpha_{1,7} \alpha_{1,8}\right)\left(\alpha_{2,2} \alpha_{2,3} \alpha_{2,4} \alpha_{2,5} \alpha_{2,6} \alpha_{2,7} \alpha_{2,8}\right) \\
\left(\alpha_{3,2} \alpha_{3,3} \alpha_{3,4} \alpha_{3,5} \alpha_{3,6} \alpha_{3,7} \alpha_{3,8}\right), \\
\widetilde{F 1_{4}}=\left(\alpha_{1,2} \alpha_{3,2}\right)\left(\alpha_{1,3} \alpha_{1,4} \alpha_{3,7} \alpha_{1,5}\right)\left(\alpha_{2,3} \alpha_{2,4} \alpha_{2,7} \alpha_{2,5}\right)\left(\alpha_{3,3} \alpha_{3,4} \alpha_{1,7} \alpha_{3,5}\right) \\
\left(\alpha_{1,6} \alpha_{1,8} \alpha_{3,6} \alpha_{3,8}\right)\left(\alpha_{2,6} \alpha_{2,8}\right), \\
\widetilde{T}_{12}=\left(\alpha_{1,1} \alpha_{1,2}\right)\left(\alpha_{3,1} \alpha_{3,2}\right)\left(\alpha_{1,3} \alpha_{1,7}\right)\left(\alpha_{3,3} \alpha_{3,7}\right)\left(\alpha_{1,4} \alpha_{1,5}\right)\left(\alpha_{3,4} \alpha_{3,5}\right) \\
\left(\alpha_{1,6} \alpha_{1,8}\right)\left(\alpha_{3,6} \alpha_{3,8}\right)
\end{gathered}
$$

See [5, Ch. 16] and [17], [18].

Using classification by Mukai [11] and its refinement by Xiao [22] of abstract finite symplectic automorphism groups of K3, and GAP Program [6] (similarly to Hashimoto in [8]), like for $N_{23}$ and $N_{22}$ in [17] and [18], we obtain the following classification. All the time we use Theorem 1 (we use Program 5 in Section 8 based on this Theorem). 
The same we do for all other cases below. This style of classification will be more convenient for studying of degenerations of K3 than our classification in [17] and [18].

\section{Classification of KahK3 conjugacy classes for $A\left(N_{21}\right)$ :}

$\mathbf{n}=\mathbf{7 4}, H \cong L_{2}(7)(|H|=168, i=42): \operatorname{rk} N_{H}=19$ and $\left(N_{H}\right)^{*} / N_{H} \cong \mathbb{Z} / 28 \mathbb{Z} \times \mathbb{Z} / 7 \mathbb{Z}$.

$$
H_{74,1}=\left[\left(\alpha_{1,2} \alpha_{1,3} \alpha_{1,5}\right)\left(\alpha_{2,2} \alpha_{2,3} \alpha_{2,5}\right)\left(\alpha_{3,2} \alpha_{3,3} \alpha_{3,5}\right)\left(\alpha_{1,4} \alpha_{1,7} \alpha_{1,6}\right)\left(\alpha_{2,4} \alpha_{2,7} \alpha_{2,6}\right)\left(\alpha_{3,4} \alpha_{3,7} \alpha_{3,6}\right)\right. \text {, }
$$$$
\left.\left(\alpha_{1,2} \alpha_{3,2}\right)\left(\alpha_{1,3} \alpha_{3,6}\right)\left(\alpha_{2,3} \alpha_{2,6}\right)\left(\alpha_{3,3} \alpha_{1,6}\right)\left(\alpha_{1,4} \alpha_{3,4}\right)\left(\alpha_{1,7} \alpha_{1,8}\right)\left(\alpha_{2,7} \alpha_{2,8}\right)\left(\alpha_{3,7} \alpha_{3,8}\right)\right]
$$

with orbits $\quad\left\{\alpha_{1,2}, \alpha_{1,3}, \alpha_{3,2}, \alpha_{1,5}, \alpha_{3,6}, \alpha_{3,3}, \alpha_{3,4}, \alpha_{3,5}, \alpha_{1,6}, \alpha_{3,7}, \alpha_{1,4}, \alpha_{3,8}, \alpha_{1,7}, \alpha_{1,8}\right\}$, $\left\{\alpha_{2,2}, \alpha_{2,3}, \alpha_{2,5}, \alpha_{2,6}, \alpha_{2,4}, \alpha_{2,7}, \alpha_{2,8}\right\}$.

$\mathbf{n}=\mathbf{5 1}, H \cong C_{2} \times \mathfrak{S}_{4}(|H|=48, i=48): \operatorname{rk} N_{H}=18,\left(N_{H}\right)^{*} / N_{H} \cong(\mathbb{Z} / 12 \mathbb{Z})^{2} \times(\mathbb{Z} / 2 \mathbb{Z})^{2}$.

$$
H_{51,1}=\left[\left(\alpha_{1,2} \alpha_{3,2}\right)\left(\alpha_{1,3} \alpha_{3,4}\right)\left(\alpha_{2,3} \alpha_{2,4}\right)\left(\alpha_{3,3} \alpha_{1,4}\right)\left(\alpha_{1,5} \alpha_{1,7}\right)\left(\alpha_{2,5} \alpha_{2,7}\right)\left(\alpha_{3,5} \alpha_{3,7}\right)\left(\alpha_{1,6} \alpha_{3,6}\right)\right. \text {, }
$$

$\left.\left(\alpha_{1,1} \alpha_{3,1}\right)\left(\alpha_{1,3} \alpha_{3,7} \alpha_{3,3} \alpha_{1,7}\right)\left(\alpha_{2,3} \alpha_{2,7}\right)\left(\alpha_{1,4} \alpha_{3,6} \alpha_{3,5} \alpha_{3,8}\right)\left(\alpha_{2,4} \alpha_{2,6} \alpha_{2,5} \alpha_{2,8}\right)\left(\alpha_{3,4} \alpha_{1,6} \alpha_{1,5} \alpha_{1,8}\right)\right]$ with orbits $\left\{\alpha_{1,1}, \alpha_{3,1}\right\}, \quad\left\{\alpha_{1,2}, \alpha_{3,2}\right\}, \quad\left\{\alpha_{1,3}, \alpha_{3,4}, \alpha_{3,7}, \alpha_{1,6}, \alpha_{3,5}, \alpha_{3,3}, \alpha_{3,6}, \alpha_{1,5}, \alpha_{3,8}, \alpha_{1,4}\right.$, $\left.\alpha_{1,7}, \alpha_{1,8}\right\},\left\{\alpha_{2,3}, \alpha_{2,4}, \alpha_{2,7}, \alpha_{2,6}, \alpha_{2,5}, \alpha_{2,8}\right\}$;

$$
H_{51,2}=\left[\left(\alpha_{1,2} \alpha_{1,3}\right)\left(\alpha_{2,2} \alpha_{2,3}\right)\left(\alpha_{3,2} \alpha_{3,3}\right)\left(\alpha_{1,4} \alpha_{3,4}\right)\left(\alpha_{1,5} \alpha_{3,8}\right)\left(\alpha_{2,5} \alpha_{2,8}\right)\left(\alpha_{3,5} \alpha_{1,8}\right)\left(\alpha_{1,6} \alpha_{3,6}\right),\right.
$$$$
\left.\left(\alpha_{1,1} \alpha_{3,1}\right)\left(\alpha_{1,3} \alpha_{3,7} \alpha_{3,3} \alpha_{1,7}\right)\left(\alpha_{2,3} \alpha_{2,7}\right)\left(\alpha_{1,4} \alpha_{3,6} \alpha_{3,5} \alpha_{3,8}\right)\left(\alpha_{2,4} \alpha_{2,6} \alpha_{2,5} \alpha_{2,8}\right)\left(\alpha_{3,4} \alpha_{1,6} \alpha_{1,5} \alpha_{1,8}\right)\right]
$$

with orbits $\quad\left\{\alpha_{1,1}, \alpha_{3,1}\right\}, \quad\left\{\alpha_{1,2}, \alpha_{1,3}, \alpha_{3,7}, \alpha_{3,3}, \alpha_{3,2}, \alpha_{1,7}\right\}, \quad\left\{\alpha_{2,2}, \alpha_{2,3}, \alpha_{2,7}\right\}$, $\left\{\alpha_{1,4}, \alpha_{3,4}, \alpha_{3,6}, \alpha_{1,6}, \alpha_{3,5}, \alpha_{1,5}, \alpha_{1,8}, \alpha_{3,8}\right\},\left\{\alpha_{2,4}, \alpha_{2,6}, \alpha_{2,5}, \alpha_{2,8}\right\}$.

$\mathbf{n}=\mathbf{3 5}, H \cong C_{2} \times \mathfrak{A}_{4},(|H|=24, i=13):$

$$
\begin{gathered}
H_{35,1}=\left[\left(\alpha_{1,1} \alpha_{3,1}\right)\left(\alpha_{1,2} \alpha_{3,2}\right)\left(\alpha_{1,3} \alpha_{3,4} \alpha_{1,6} \alpha_{3,3} \alpha_{1,4} \alpha_{3,6}\right)\left(\alpha_{2,3} \alpha_{2,4} \alpha_{2,6}\right)\left(\alpha_{1,5} \alpha_{3,8} \alpha_{1,7} \alpha_{3,5} \alpha_{1,8} \alpha_{3,7}\right)\right. \\
\left(\alpha_{2,5} \alpha_{2,8} \alpha_{2,7}\right), \\
\left(\alpha_{1,1} \alpha_{3,1}\right)\left(\alpha_{1,2} \alpha_{3,2}\right)\left(\alpha_{1,3} \alpha_{1,4} \alpha_{1,8} \alpha_{3,3} \alpha_{3,4} \alpha_{3,8}\right)\left(\alpha_{2,3} \alpha_{2,4} \alpha_{2,8}\right)\left(\alpha_{1,5} \alpha_{1,6} \alpha_{3,7} \alpha_{3,5} \alpha_{3,6} \alpha_{1,7}\right) \\
\left.\left(\alpha_{2,5} \alpha_{2,6} \alpha_{2,7}\right)\right]
\end{gathered}
$$

with $\operatorname{Clos}\left(H_{35,1}\right)=H_{51,1}$ above;

$$
H_{35,2}=\left[\left(\alpha_{1,1} \alpha_{3,1}\right)\left(\alpha_{1,2} \alpha_{1,3} \alpha_{3,7} \alpha_{3,2} \alpha_{3,3} \alpha_{1,7}\right)\left(\alpha_{2,2} \alpha_{2,3} \alpha_{2,7}\right)\left(\alpha_{1,4} \alpha_{1,6} \alpha_{3,8} \alpha_{3,4} \alpha_{3,6} \alpha_{1,8}\right)\right.
$$




$$
\begin{gathered}
\left(\alpha_{2,4} \alpha_{2,6} \alpha_{2,8}\right)\left(\alpha_{1,5} \alpha_{3,5}\right) \\
\left(\alpha_{1,1} \alpha_{3,1}\right)\left(\alpha_{1,2} \alpha_{1,3} \alpha_{1,7} \alpha_{3,2} \alpha_{3,3} \alpha_{3,7}\right)\left(\alpha_{2,2} \alpha_{2,3} \alpha_{2,7}\right)\left(\alpha_{1,4} \alpha_{1,5} \alpha_{3,6} \alpha_{3,4} \alpha_{3,5} \alpha_{1,6}\right) \\
\left.\left(\alpha_{2,4} \alpha_{2,5} \alpha_{2,6}\right)\left(\alpha_{1,8} \alpha_{3,8}\right)\right]
\end{gathered}
$$

with $\operatorname{Clos}\left(H_{35,2}\right)=H_{51,2}$ above.

$\mathbf{n}=34, H \cong \mathfrak{S}_{4}(|H|=24, i=12): \operatorname{rk} N_{H}=17$ and $\left(N_{H}\right)^{*} / N_{H} \cong(\mathbb{Z} / 12 \mathbb{Z})^{2} \times \mathbb{Z} / 4 \mathbb{Z}$.

$$
\begin{gathered}
H_{34,1}=\left[\left(\alpha_{1,3} \alpha_{3,4} \alpha_{1,8}\right)\left(\alpha_{2,3} \alpha_{2,4} \alpha_{2,8}\right)\left(\alpha_{3,3} \alpha_{1,4} \alpha_{3,8}\right)\left(\alpha_{1,5} \alpha_{3,6} \alpha_{3,7}\right)\right. \\
\left(\alpha_{2,5} \alpha_{2,6} \alpha_{2,7}\right)\left(\alpha_{3,5} \alpha_{1,6} \alpha_{1,7}\right) \\
\left(\alpha_{1,1} \alpha_{3,1}\right)\left(\alpha_{1,3} \alpha_{3,7} \alpha_{3,3} \alpha_{1,7}\right)\left(\alpha_{2,3} \alpha_{2,7}\right)\left(\alpha_{1,4} \alpha_{3,6} \alpha_{3,5} \alpha_{3,8}\right) \\
\left.\left(\alpha_{2,4} \alpha_{2,6} \alpha_{2,5} \alpha_{2,8}\right)\left(\alpha_{3,4} \alpha_{1,6} \alpha_{1,5} \alpha_{1,8}\right)\right]
\end{gathered}
$$

with orbits $\quad\left\{\alpha_{1,1}, \alpha_{3,1}\right\}, \quad\left\{\alpha_{1,3}, \alpha_{3,4}, \alpha_{3,7}, \alpha_{1,8}, \alpha_{1,6}, \alpha_{1,5}, \alpha_{3,3}, \alpha_{1,7}, \alpha_{3,6}, \alpha_{1,4}, \alpha_{3,5}, \alpha_{3,8}\right\}$, $\left\{\alpha_{2,3}, \alpha_{2,4}, \alpha_{2,7}, \alpha_{2,8}, \alpha_{2,6}, \alpha_{2,5}\right\}$;

$$
\begin{gathered}
H_{34,2}=\left[\left(\alpha_{1,2} \alpha_{1,3} \alpha_{3,7}\right)\left(\alpha_{2,2} \alpha_{2,3} \alpha_{2,7}\right)\left(\alpha_{3,2} \alpha_{3,3} \alpha_{1,7}\right)\left(\alpha_{1,4} \alpha_{3,8} \alpha_{3,5}\right)\right. \\
\left(\alpha_{2,4} \alpha_{2,8} \alpha_{2,5}\right)\left(\alpha_{3,4} \alpha_{1,8} \alpha_{1,5}\right) \\
\left(\alpha_{1,1} \alpha_{3,1}\right)\left(\alpha_{1,3} \alpha_{3,7} \alpha_{3,3} \alpha_{1,7}\right)\left(\alpha_{2,3} \alpha_{2,7}\right)\left(\alpha_{1,4} \alpha_{3,6} \alpha_{3,5} \alpha_{3,8}\right) \\
\left.\left(\alpha_{2,4} \alpha_{2,6} \alpha_{2,5} \alpha_{2,8}\right)\left(\alpha_{3,4} \alpha_{1,6} \alpha_{1,5} \alpha_{1,8}\right)\right]
\end{gathered}
$$

with orbits $\left\{\alpha_{1,1}, \alpha_{3,1}\right\}, \quad\left\{\alpha_{1,2}, \alpha_{1,3}, \alpha_{3,7}, \alpha_{3,3}, \alpha_{1,7}, \alpha_{3,2}\right\}, \quad\left\{\alpha_{2,2}, \alpha_{2,3}, \alpha_{2,7}\right\}, \quad\left\{\alpha_{1,4}, \alpha_{3,8}, \alpha_{3,6}\right.$, $\left.\alpha_{3,5}\right\},\left\{\alpha_{2,4}, \alpha_{2,8}, \alpha_{2,6}, \alpha_{2,5}\right\},\left\{\alpha_{3,4}, \alpha_{1,8}, \alpha_{1,6}, \alpha_{1,5}\right\}$;

$$
\begin{gathered}
H_{34,3}=\left[\left(\alpha_{1,1} \alpha_{1,3} \alpha_{1,7}\right)\left(\alpha_{2,1} \alpha_{2,3} \alpha_{2,7}\right)\left(\alpha_{3,1} \alpha_{3,3} \alpha_{3,7}\right)\left(\alpha_{1,4} \alpha_{1,8} \alpha_{1,6}\right)\right. \\
\left(\alpha_{2,4} \alpha_{2,8} \alpha_{2,6}\right)\left(\alpha_{3,4} \alpha_{3,8} \alpha_{3,6}\right), \\
\left(\alpha_{1,1} \alpha_{3,1}\right)\left(\alpha_{1,3} \alpha_{3,7} \alpha_{3,3} \alpha_{1,7}\right)\left(\alpha_{2,3} \alpha_{2,7}\right)\left(\alpha_{1,4} \alpha_{3,6} \alpha_{3,5} \alpha_{3,8}\right) \\
\left.\left(\alpha_{2,4} \alpha_{2,6} \alpha_{2,5} \alpha_{2,8}\right)\left(\alpha_{3,4} \alpha_{1,6} \alpha_{1,5} \alpha_{1,8}\right)\right]
\end{gathered}
$$

with orbits $\left\{\alpha_{1,1}, \alpha_{1,3}, \alpha_{3,1}, \alpha_{1,7}, \alpha_{3,7}, \alpha_{3,3}\right\}, \quad\left\{\alpha_{2,1}, \alpha_{2,3}, \alpha_{2,7}\right\}, \quad\left\{\alpha_{1,4}, \alpha_{1,8}, \alpha_{3,6}, \alpha_{1,6}, \alpha_{3,4}, \alpha_{3,5}\right.$, $\left.\alpha_{1,5}, \alpha_{3,8}\right\},\left\{\alpha_{2,4}, \alpha_{2,8}, \alpha_{2,6}, \alpha_{2,5}\right\}$.

$\mathbf{n}=33, H \cong C_{7} \rtimes C_{3}(|H|=21, i=1): \operatorname{rk} N_{H}=18$ and $\left(N_{H}\right)^{*} / N_{H} \cong(\mathbb{Z} / 7 \mathbb{Z})^{3}$.

$$
H_{33,1}=\left[\left(\alpha_{1,3} \alpha_{1,5} \alpha_{3,8}\right)\left(\alpha_{2,3} \alpha_{2,5} \alpha_{2,8}\right)\left(\alpha_{3,3} \alpha_{3,5} \alpha_{1,8}\right)\left(\alpha_{1,4} \alpha_{3,6} \alpha_{1,7}\right)\right.
$$




$$
\begin{gathered}
\left(\alpha_{2,4} \alpha_{2,6} \alpha_{2,7}\right)\left(\alpha_{3,4} \alpha_{1,6} \alpha_{3,7}\right) \\
\left(\alpha_{1,2} \alpha_{3,4} \alpha_{1,6} \alpha_{3,5} \alpha_{3,7} \alpha_{3,3} \alpha_{1,8}\right)\left(\alpha_{2,2} \alpha_{2,4} \alpha_{2,6} \alpha_{2,5} \alpha_{2,7} \alpha_{2,3} \alpha_{2,8}\right) \\
\left.\left(\alpha_{3,2} \alpha_{1,4} \alpha_{3,6} \alpha_{1,5} \alpha_{1,7} \alpha_{1,3} \alpha_{3,8}\right)\right]
\end{gathered}
$$

with orbits $\quad\left\{\alpha_{1,2}, \alpha_{3,4}, \alpha_{1,6}, \alpha_{3,7}, \alpha_{3,5}, \alpha_{3,3}, \alpha_{1,8}\right\}, \quad\left\{\alpha_{2,2}, \alpha_{2,4}, \alpha_{2,6}, \alpha_{2,7}, \alpha_{2,5}, \alpha_{2,3}, \alpha_{2,8}\right\}$, $\left\{\alpha_{3,2}, \alpha_{1,4}, \alpha_{3,6}, \alpha_{1,7}, \alpha_{1,5}, \alpha_{1,3}, \alpha_{3,8}\right\}$.

$\mathbf{n}=\mathbf{2 2}, H \cong C_{2} \times D_{8}(|H|=16, i=11): \operatorname{rk} N_{H}=16,\left(N_{H}\right)^{*} / N_{H} \cong(\mathbb{Z} / 4 \mathbb{Z})^{4} \times(\mathbb{Z} / 2 \mathbb{Z})^{2}$.

$$
\begin{gathered}
H_{22,1}=\left[\left(\alpha_{1,3} \alpha_{3,3}\right)\left(\alpha_{1,4} \alpha_{3,5}\right)\left(\alpha_{2,4} \alpha_{2,5}\right)\left(\alpha_{3,4} \alpha_{1,5}\right)\left(\alpha_{1,6} \alpha_{1,8}\right)\right. \\
\left(\alpha_{2,6} \alpha_{2,8}\right)\left(\alpha_{3,6} \alpha_{3,8}\right)\left(\alpha_{1,7} \alpha_{3,7}\right) \\
\left(\alpha_{1,2} \alpha_{3,2}\right)\left(\alpha_{1,3} \alpha_{3,6}\right)\left(\alpha_{2,3} \alpha_{2,6}\right)\left(\alpha_{3,3} \alpha_{1,6}\right)\left(\alpha_{1,4} \alpha_{3,4}\right)\left(\alpha_{1,7} \alpha_{1,8}\right) \\
\left(\alpha_{2,7} \alpha_{2,8}\right)\left(\alpha_{3,7} \alpha_{3,8}\right) \\
\left(\alpha_{1,1} \alpha_{3,1}\right)\left(\alpha_{1,2} \alpha_{3,2}\right)\left(\alpha_{1,3} \alpha_{3,3}\right)\left(\alpha_{1,4} \alpha_{3,4}\right)\left(\alpha_{1,5} \alpha_{3,5}\right)\left(\alpha_{1,6} \alpha_{3,6}\right) \\
\left.\left(\alpha_{1,7} \alpha_{3,7}\right)\left(\alpha_{1,8} \alpha_{3,8}\right)\right]
\end{gathered}
$$

with orbits $\quad\left\{\alpha_{1,1}, \alpha_{3,1}\right\}, \quad\left\{\alpha_{1,2}, \alpha_{3,2}\right\}, \quad\left\{\alpha_{1,3}, \alpha_{3,3}, \alpha_{3,6}, \alpha_{1,6}, \alpha_{3,8}, \alpha_{1,8}, \alpha_{3,7}, \alpha_{1,7}\right\}$, $\left\{\alpha_{2,3}, \alpha_{2,6}, \alpha_{2,8}, \alpha_{2,7}\right\},\left\{\alpha_{1,4}, \alpha_{3,5}, \alpha_{3,4}, \alpha_{1,5}\right\},\left\{\alpha_{2,4}, \alpha_{2,5}\right\}$.

$\mathbf{n}=\mathbf{1 8}, H \cong D_{12}(|H|=12, i=4): \operatorname{rk} N_{H}=16$ and $\left(N_{H}\right)^{*} / N_{H} \cong(\mathbb{Z} / 6 \mathbb{Z})^{4}$.

$$
\begin{gathered}
H_{18,1}=\left[\left(\alpha_{1,2} \alpha_{3,2}\right)\left(\alpha_{1,3} \alpha_{3,3}\right)\left(\alpha_{1,4} \alpha_{3,6}\right)\left(\alpha_{2,4} \alpha_{2,6}\right)\left(\alpha_{3,4} \alpha_{1,6}\right)\left(\alpha_{1,5} \alpha_{1,8}\right)\right. \\
\left(\alpha_{2,5} \alpha_{2,8}\right)\left(\alpha_{3,5} \alpha_{3,8}\right), \\
\left(\alpha_{1,1} \alpha_{3,1}\right)\left(\alpha_{1,2} \alpha_{3,2}\right)\left(\alpha_{1,3} \alpha_{3,4} \alpha_{1,6} \alpha_{3,3} \alpha_{1,4} \alpha_{3,6}\right)\left(\alpha_{2,3} \alpha_{2,4} \alpha_{2,6}\right) \\
\left.\left(\alpha_{1,5} \alpha_{3,8} \alpha_{1,7} \alpha_{3,5} \alpha_{1,8} \alpha_{3,7}\right)\left(\alpha_{2,5} \alpha_{2,8} \alpha_{2,7}\right)\right]
\end{gathered}
$$

with orbits $\left\{\alpha_{1,1}, \alpha_{3,1}\right\}, \quad\left\{\alpha_{1,2}, \alpha_{3,2}\right\}, \quad\left\{\alpha_{1,3}, \alpha_{3,3}, \alpha_{3,4}, \alpha_{1,4}, \alpha_{1,6}, \alpha_{3,6}\right\}, \quad\left\{\alpha_{2,3}, \alpha_{2,4}, \alpha_{2,6}\right\}$, $\left\{\alpha_{1,5}, \alpha_{1,8}, \alpha_{3,8}, \alpha_{3,7}, \alpha_{3,5}, \alpha_{1,7}\right\},\left\{\alpha_{2,5}, \alpha_{2,8}, \alpha_{2,7}\right\}$.

$\mathbf{n}=\mathbf{1 7}, H \cong \mathfrak{A}_{4}(|H|=12, i=3): \operatorname{rk} N_{H}=16$ and $\left(N_{H}\right)^{*} / N_{H} \cong(\mathbb{Z} / 12 \mathbb{Z})^{2} \times(\mathbb{Z} / 2 \mathbb{Z})^{2}$.

$$
\begin{gathered}
H_{17,1}=\left[\left(\alpha_{1,2} \alpha_{1,3} \alpha_{3,8}\right)\left(\alpha_{2,2} \alpha_{2,3} \alpha_{2,8}\right)\left(\alpha_{3,2} \alpha_{3,3} \alpha_{1,8}\right)\left(\alpha_{1,4} \alpha_{1,6} \alpha_{3,7}\right)\right. \\
\left(\alpha_{2,4} \alpha_{2,6} \alpha_{2,7}\right)\left(\alpha_{3,4} \alpha_{3,6} \alpha_{1,7}\right), \\
\left(\alpha_{1,2} \alpha_{3,2}\right)\left(\alpha_{1,3} \alpha_{3,6}\right)\left(\alpha_{2,3} \alpha_{2,6}\right)\left(\alpha_{3,3} \alpha_{1,6}\right)\left(\alpha_{1,4} \alpha_{3,4}\right)\left(\alpha_{1,7} \alpha_{1,8}\right) \\
\left.\left(\alpha_{2,7} \alpha_{2,8}\right)\left(\alpha_{3,7} \alpha_{3,8}\right)\right]
\end{gathered}
$$


with $\quad$ orbits $\quad\left\{\alpha_{1,2}, \alpha_{1,3}, \alpha_{3,2}, \alpha_{3,8}, \alpha_{3,6}, \alpha_{3,3}, \alpha_{3,7}, \alpha_{1,7}, \alpha_{1,8}, \alpha_{1,6}, \alpha_{1,4}, \alpha_{3,4}\right\}, \quad\left\{\alpha_{2,2}, \alpha_{2,3}, \alpha_{2,8}\right.$, $\left.\alpha_{2,6}, \alpha_{2,7}, \alpha_{2,4}\right\}$;

$$
\begin{gathered}
H_{17,2}=\left[\left(\alpha_{1,2} \alpha_{1,4} \alpha_{3,5}\right)\left(\alpha_{2,2} \alpha_{2,4} \alpha_{2,5}\right)\left(\alpha_{3,2} \alpha_{3,4} \alpha_{1,5}\right)\left(\alpha_{1,6} \alpha_{3,7} \alpha_{3,8}\right)\right. \\
\left(\alpha_{2,6} \alpha_{2,7} \alpha_{2,8}\right)\left(\alpha_{3,6} \alpha_{1,7} \alpha_{1,8}\right), \\
\left(\alpha_{1,2} \alpha_{3,2}\right)\left(\alpha_{1,3} \alpha_{3,6}\right)\left(\alpha_{2,3} \alpha_{2,6}\right)\left(\alpha_{3,3} \alpha_{1,6}\right)\left(\alpha_{1,4} \alpha_{3,4}\right)\left(\alpha_{1,7} \alpha_{1,8}\right) \\
\left.\left(\alpha_{2,7} \alpha_{2,8}\right)\left(\alpha_{3,7} \alpha_{3,8}\right)\right]
\end{gathered}
$$

with orbits $\left\{\alpha_{1,2}, \alpha_{1,4}, \alpha_{3,2}, \alpha_{3,5}, \alpha_{3,4}, \alpha_{1,5}\right\},\left\{\alpha_{2,2}, \alpha_{2,4}, \alpha_{2,5}\right\},\left\{\alpha_{1,3}, \alpha_{3,6}, \alpha_{1,7}, \alpha_{1,8}\right\},\left\{\alpha_{2,3}, \alpha_{2,6}\right.$, $\left.\alpha_{2,7}, \alpha_{2,8}\right\},\left\{\alpha_{3,3}, \alpha_{1,6}, \alpha_{3,7}, \alpha_{3,8}\right\}$.

$\mathbf{n}=\mathbf{1 1}, H \cong C_{2} \times C_{4}(|H|=8, i=2):$

$$
\begin{gathered}
H_{11,1}=\left[\left(\alpha_{1,1} \alpha_{3,1}\right)\left(\alpha_{1,2} \alpha_{3,2}\right)\left(\alpha_{1,3} \alpha_{3,3}\right)\left(\alpha_{1,4} \alpha_{3,4}\right)\left(\alpha_{1,5} \alpha_{3,5}\right)\left(\alpha_{1,6} \alpha_{3,6}\right)\right. \\
\left(\alpha_{1,7} \alpha_{3,7}\right)\left(\alpha_{1,8} \alpha_{3,8}\right), \\
\left(\alpha_{1,2} \alpha_{3,2}\right)\left(\alpha_{1,3} \alpha_{1,6} \alpha_{1,7} \alpha_{3,8}\right)\left(\alpha_{2,3} \alpha_{2,6} \alpha_{2,7} \alpha_{2,8}\right)\left(\alpha_{3,3} \alpha_{3,6} \alpha_{3,7} \alpha_{1,8}\right) \\
\left.\left(\alpha_{1,4} \alpha_{3,5} \alpha_{3,4} \alpha_{1,5}\right)\left(\alpha_{2,4} \alpha_{2,5}\right)\right]
\end{gathered}
$$

with $\operatorname{Clos}\left(H_{11,1}\right)=H_{22,1}$ above.

$\mathbf{n}=\mathbf{1 0}, H \cong D_{8}(|H|=8, i=3): \operatorname{rk} N_{H}=15$ and $\left(N_{H}\right)^{*} / N_{H} \cong(\mathbb{Z} / 4 \mathbb{Z})^{5}$.

$$
\begin{gathered}
H_{10,1}=\left[\left(\alpha_{1,3} \alpha_{3,3}\right)\left(\alpha_{1,4} \alpha_{3,5}\right)\left(\alpha_{2,4} \alpha_{2,5}\right)\left(\alpha_{3,4} \alpha_{1,5}\right)\left(\alpha_{1,6} \alpha_{1,8}\right)\right. \\
\left(\alpha_{2,6} \alpha_{2,8}\right)\left(\alpha_{3,6} \alpha_{3,8}\right)\left(\alpha_{1,7} \alpha_{3,7}\right), \\
\left(\alpha_{1,2} \alpha_{3,2}\right)\left(\alpha_{1,3} \alpha_{3,6}\right)\left(\alpha_{2,3} \alpha_{2,6}\right)\left(\alpha_{3,3} \alpha_{1,6}\right)\left(\alpha_{1,4} \alpha_{3,4}\right)\left(\alpha_{1,7} \alpha_{1,8}\right) \\
\left.\left(\alpha_{2,7} \alpha_{2,8}\right)\left(\alpha_{3,7} \alpha_{3,8}\right)\right]
\end{gathered}
$$

with $\quad$ orbits $\quad\left\{\alpha_{1,2}, \alpha_{3,2}\right\}, \quad\left\{\alpha_{1,3}, \alpha_{3,3}, \alpha_{3,6}, \alpha_{1,6}, \alpha_{3,8}, \alpha_{1,8}, \alpha_{3,7}, \alpha_{1,7}\right\}, \quad\left\{\alpha_{2,3}, \alpha_{2,6}, \alpha_{2,8}, \alpha_{2,7}\right\}$, $\left\{\alpha_{1,4}, \alpha_{3,5}, \alpha_{3,4}, \alpha_{1,5}\right\},\left\{\alpha_{2,4}, \alpha_{2,5}\right\}$

$$
\begin{gathered}
H_{10,2}=\left[\left(\alpha_{1,1} \alpha_{3,1}\right)\left(\alpha_{1,2} \alpha_{3,2}\right)\left(\alpha_{1,4} \alpha_{1,5}\right)\left(\alpha_{2,4} \alpha_{2,5}\right)\left(\alpha_{3,4} \alpha_{3,5}\right)\left(\alpha_{1,6} \alpha_{3,8}\right)\right. \\
\left(\alpha_{2,6} \alpha_{2,8}\right)\left(\alpha_{3,6} \alpha_{1,8}\right), \\
\left(\alpha_{1,2} \alpha_{3,2}\right)\left(\alpha_{1,3} \alpha_{3,6}\right)\left(\alpha_{2,3} \alpha_{2,6}\right)\left(\alpha_{3,3} \alpha_{1,6}\right)\left(\alpha_{1,4} \alpha_{3,4}\right)\left(\alpha_{1,7} \alpha_{1,8}\right) \\
\left.\left(\alpha_{2,7} \alpha_{2,8}\right)\left(\alpha_{3,7} \alpha_{3,8}\right)\right]
\end{gathered}
$$


with orbits $\quad\left\{\alpha_{1,1}, \alpha_{3,1}\right\}, \quad\left\{\alpha_{1,2}, \alpha_{3,2}\right\}, \quad\left\{\alpha_{1,3}, \alpha_{3,6}, \alpha_{1,8}, \alpha_{1,7}\right\}, \quad\left\{\alpha_{2,3}, \alpha_{2,6}, \alpha_{2,8}, \alpha_{2,7}\right\}$, $\left\{\alpha_{3,3}, \alpha_{1,6}, \alpha_{3,8}, \alpha_{3,7}\right\},\left\{\alpha_{1,4}, \alpha_{1,5}, \alpha_{3,4}, \alpha_{3,5}\right\},\left\{\alpha_{2,4}, \alpha_{2,5}\right\}$.

$\mathbf{n}=\mathbf{9}, H \cong C_{2}^{3}(|H|=8, i=5): \operatorname{rk} N_{H}=14,\left(N_{H}\right)^{*} / N_{H} \cong(\mathbb{Z} / 4 \mathbb{Z})^{2} \times(\mathbb{Z} / 2 \mathbb{Z})^{6}$.

$$
\begin{gathered}
H_{9,1}=\left[\left(\alpha_{1,1} \alpha_{3,1}\right)\left(\alpha_{1,2} \alpha_{3,2}\right)\left(\alpha_{1,3} \alpha_{3,3}\right)\left(\alpha_{1,4} \alpha_{3,4}\right)\left(\alpha_{1,5} \alpha_{3,5}\right)\right. \\
\left(\alpha_{1,6} \alpha_{3,6}\right)\left(\alpha_{1,7} \alpha_{3,7}\right)\left(\alpha_{1,8} \alpha_{3,8}\right), \\
\left(\alpha_{1,3} \alpha_{1,7}\right)\left(\alpha_{2,3} \alpha_{2,7}\right)\left(\alpha_{3,3} \alpha_{3,7}\right)\left(\alpha_{1,4} \alpha_{3,4}\right)\left(\alpha_{1,5} \alpha_{3,5}\right) \\
\left(\alpha_{1,6} \alpha_{3,8}\right)\left(\alpha_{2,6} \alpha_{2,8}\right)\left(\alpha_{3,6} \alpha_{1,8}\right), \\
\left(\alpha_{41,2} \alpha_{63,2}\right)\left(\alpha_{71,3} \alpha_{183,6}\right)\left(\alpha_{2,3} \alpha_{2,6}\right)\left(\alpha_{3,3} \alpha_{1,6}\right)\left(\alpha_{1,4} \alpha_{3,4}\right) \\
\left.\left(\alpha_{1,7} \alpha_{1,8}\right)\left(\alpha_{2,7} \alpha_{2,8}\right)\left(\alpha_{3,7} \alpha_{3,8}\right)\right]
\end{gathered}
$$

with orbits $\quad\left\{\alpha_{1,1}, \alpha_{3,1}\right\}, \quad\left\{\alpha_{1,2}, \alpha_{3,2}\right\}, \quad\left\{\alpha_{1,3}, \alpha_{3,3}, \alpha_{1,7}, \alpha_{3,6}, \alpha_{3,7}, \alpha_{1,6}, \alpha_{1,8}, \alpha_{3,8}\right\}$, $\left\{\alpha_{2,3}, \alpha_{2,7}, \alpha_{2,6}, \alpha_{2,8}\right\},\left\{\alpha_{1,4}, \alpha_{3,4}\right\},\left\{\alpha_{1,5}, \alpha_{3,5}\right\}$;

$$
\begin{gathered}
H_{9,2}=\left[\left(\alpha_{1,1} \alpha_{3,1}\right)\left(\alpha_{1,2} \alpha_{3,2}\right)\left(\alpha_{1,3} \alpha_{3,3}\right)\left(\alpha_{1,4} \alpha_{3,4}\right)\left(\alpha_{1,5} \alpha_{3,5}\right)\right. \\
\left(\alpha_{1,6} \alpha_{3,6}\right)\left(\alpha_{1,7} \alpha_{3,7}\right)\left(\alpha_{1,8} \alpha_{3,8}\right), \\
\left(\alpha_{1,2} \alpha_{1,4}\right)\left(\alpha_{2,2} \alpha_{2,4}\right)\left(\alpha_{3,2} \alpha_{3,4}\right)\left(\alpha_{1,3} \alpha_{3,3}\right)\left(\alpha_{1,6} \alpha_{3,6}\right) \\
\left(\alpha_{1,7} \alpha_{3,8}\right)\left(\alpha_{2,7} \alpha_{2,8}\right)\left(\alpha_{3,7} \alpha_{1,8}\right), \\
\left(\alpha_{1,2} \alpha_{3,2}\right)\left(\alpha_{1,3} \alpha_{3,6}\right)\left(\alpha_{2,3} \alpha_{2,6}\right)\left(\alpha_{3,3} \alpha_{1,6}\right)\left(\alpha_{1,4} \alpha_{3,4}\right) \\
\left.\left(\alpha_{1,7} \alpha_{1,8}\right)\left(\alpha_{2,7} \alpha_{2,8}\right)\left(\alpha_{3,7} \alpha_{3,8}\right)\right]
\end{gathered}
$$

with orbits $\quad\left\{\alpha_{1,1}, \alpha_{3,1}\right\}, \quad\left\{\alpha_{1,2}, \alpha_{3,2}, \alpha_{1,4}, \alpha_{3,4}\right\}, \quad\left\{\alpha_{2,2}, \alpha_{2,4}\right\}, \quad\left\{\alpha_{1,3}, \alpha_{3,3}, \alpha_{3,6}, \alpha_{1,6}\right\}$, $\left\{\alpha_{2,3}, \alpha_{2,6}\right\},\left\{\alpha_{1,5}, \alpha_{3,5}\right\},\left\{\alpha_{1,7}, \alpha_{3,7}, \alpha_{3,8}, \alpha_{1,8}\right\},\left\{\alpha_{2,7}, \alpha_{2,8}\right\}$.

$\mathbf{n}=8, H \cong C_{7}(|H|=7, i=1)$ :

$$
\begin{gathered}
H_{8,1}=\left[\left(\alpha_{1,2} \alpha_{3,4} \alpha_{1,6} \alpha_{3,5} \alpha_{3,7} \alpha_{3,3} \alpha_{1,8}\right)\left(\alpha_{2,2} \alpha_{2,4} \alpha_{2,6} \alpha_{2,5} \alpha_{2,7} \alpha_{2,3} \alpha_{2,8}\right)\right. \\
\left.\left(\alpha_{3,2} \alpha_{1,4} \alpha_{3,6} \alpha_{1,5} \alpha_{1,7} \alpha_{1,3} \alpha_{3,8}\right)\right]
\end{gathered}
$$

with $\operatorname{Clos}\left(H_{8,1}\right)=H_{33,1}$ above.

$$
\begin{gathered}
\mathbf{n}=\mathbf{7}, H \cong C_{6}(|H|=6, i=2): \\
H_{7,1}=\left[\left(\alpha_{1,1} \alpha_{3,1}\right)\left(\alpha_{1,2} \alpha_{3,2}\right)\left(\alpha_{1,3} \alpha_{3,4} \alpha_{1,6} \alpha_{3,3} \alpha_{1,4} \alpha_{3,6}\right)\left(\alpha_{2,3} \alpha_{2,4} \alpha_{2,6}\right)\right. \\
\left.\left(\alpha_{1,5} \alpha_{3,8} \alpha_{1,7} \alpha_{3,5} \alpha_{1,8} \alpha_{3,7}\right)\left(\alpha_{2,5} \alpha_{2,8} \alpha_{2,7}\right)\right]
\end{gathered}
$$


with $\operatorname{Clos}\left(H_{7,1}\right)=H_{18,1}$ above.

$\mathbf{n}=\mathbf{6}, H \cong D_{6}(|H|=6, i=1): \operatorname{rk} N_{H}=14$ and $\left(N_{H}\right)^{*} / N_{H} \cong(\mathbb{Z} / 6 \mathbb{Z})^{2} \times(\mathbb{Z} / 3 \mathbb{Z})^{3}$.

$$
\begin{gathered}
H_{6,1}=\left[\left(\alpha_{1,2} \alpha_{3,2}\right)\left(\alpha_{1,3} \alpha_{3,3}\right)\left(\alpha_{1,4} \alpha_{3,6}\right)\left(\alpha_{2,4} \alpha_{2,6}\right)\left(\alpha_{3,4} \alpha_{1,6}\right)\right. \\
\left(\alpha_{1,5} \alpha_{1,8}\right)\left(\alpha_{2,5} \alpha_{2,8}\right)\left(\alpha_{3,5} \alpha_{3,8}\right), \\
\left(\alpha_{1,3} \alpha_{1,4} \alpha_{1,6}\right)\left(\alpha_{2,3} \alpha_{2,4} \alpha_{2,6}\right)\left(\alpha_{3,3} \alpha_{3,4} \alpha_{3,6}\right)\left(\alpha_{1,5} \alpha_{1,8} \alpha_{1,7}\right) \\
\left.\left(\alpha_{2,5} \alpha_{2,8} \alpha_{2,7}\right)\left(\alpha_{3,5} \alpha_{3,8} \alpha_{3,7}\right)\right]
\end{gathered}
$$

with orbits $\left\{\alpha_{1,2}, \alpha_{3,2}\right\},\left\{\alpha_{1,3}, \alpha_{3,3}, \alpha_{1,4}, \alpha_{3,4}, \alpha_{3,6}, \alpha_{1,6}\right\},\left\{\alpha_{2,3}, \alpha_{2,4}, \alpha_{2,6}\right\},\left\{\alpha_{1,5}, \alpha_{1,8}, \alpha_{1,7}\right\}$, $\left\{\alpha_{2,5}, \alpha_{2,8}, \alpha_{2,7}\right\},\left\{\alpha_{3,5}, \alpha_{3,8}, \alpha_{3,7}\right\}$.

$\mathbf{n}=4, H \cong C_{4}(|H|=4, i=1): \operatorname{rk} N_{H}=14$ and $\left(N_{H}\right)^{*} / N_{H} \cong(\mathbb{Z} / 4 \mathbb{Z})^{4} \times(\mathbb{Z} / 2 \mathbb{Z})^{2}$.

$$
\begin{gathered}
H_{4,1}=\left[\left(\alpha_{1,1} \alpha_{3,1}\right)\left(\alpha_{1,3} \alpha_{3,7}, \alpha_{3,3} \alpha_{1,7}\right)\left(\alpha_{2,3} \alpha_{2,7}\right)\left(\alpha_{1,4} \alpha_{3,6} \alpha_{3,5} \alpha_{3,8}\right)\right. \\
\left.\left(\alpha_{2,4} \alpha_{2,6} \alpha_{2,5} \alpha_{2,8}\right)\left(\alpha_{3,4} \alpha_{1,6} \alpha_{1,5} \alpha_{1,8}\right)\right]
\end{gathered}
$$

with orbits $\quad\left\{\alpha_{1,1}, \alpha_{3,1}\right\}, \quad\left\{\alpha_{1,3}, \alpha_{3,7}, \alpha_{3,3}, \alpha_{1,7}\right\}, \quad\left\{\alpha_{2,3}, \alpha_{2,7}\right\}, \quad\left\{\alpha_{1,4}, \alpha_{3,6}, \alpha_{3,5}, \alpha_{3,8}\right\}$, $\left\{\alpha_{2,4}, \alpha_{2,6}, \alpha_{2,5}, \alpha_{2,8}\right\},\left\{\alpha_{3,4}, \alpha_{1,6}, \alpha_{1,5}, \alpha_{1,8}\right\}$.

$\mathbf{n}=\mathbf{3}, H \cong C_{2}^{2}(|H|=4, i=2): \operatorname{rk} N_{H}=12$ and $\left(N_{H}\right)^{*} / N_{H} \cong(\mathbb{Z} / 4 \mathbb{Z})^{2} \times(\mathbb{Z} / 2 \mathbb{Z})^{6}$.

$$
\begin{gathered}
H_{3,1}=\left[\left(\alpha_{1,1} \alpha_{3,1}\right)\left(\alpha_{1,2} \alpha_{3,2}\right)\left(\alpha_{1,3} \alpha_{3,3}\right)\left(\alpha_{1,4} \alpha_{3,4}\right)\left(\alpha_{1,5} \alpha_{3,5}\right)\left(\alpha_{1,6} \alpha_{3,6}\right)\right. \\
\left(\alpha_{1,7} \alpha_{3,7}\right)\left(\alpha_{1,8} \alpha_{3,8}\right), \\
\left(\alpha_{1,2} \alpha_{3,2}\right)\left(\alpha_{1,3} \alpha_{3,6}\right)\left(\alpha_{2,3} \alpha_{2,6}\right)\left(\alpha_{3,3} \alpha_{1,6}\right)\left(\alpha_{1,4} \alpha_{3,4}\right)\left(\alpha_{1,7} \alpha_{1,8}\right) \\
\left.\left(\alpha_{2,7} \alpha_{2,8}\right)\left(\alpha_{3,7} \alpha_{3,8}\right)\right]
\end{gathered}
$$

with orbits $\left\{\alpha_{1,1}, \alpha_{3,1}\right\}, \quad\left\{\alpha_{1,2}, \alpha_{3,2}\right\}, \quad\left\{\alpha_{1,3}, \alpha_{3,3}, \alpha_{3,6}, \alpha_{1,6}\right\}, \quad\left\{\alpha_{2,3}, \alpha_{2,6}\right\}, \quad\left\{\alpha_{1,4}, \alpha_{3,4}\right\}$, $\left\{\alpha_{1,5}, \alpha_{3,5}\right\},\left\{\alpha_{1,7}, \alpha_{3,7}, \alpha_{1,8}, \alpha_{3,8}\right\},\left\{\alpha_{2,7}, \alpha_{2,8}\right\}$;

$$
\begin{gathered}
H_{3,2}=\left[\left(\alpha_{1,3} \alpha_{1,7}\right)\left(\alpha_{2,3} \alpha_{2,7}\right)\left(\alpha_{3,3} \alpha_{3,7}\right)\left(\alpha_{1,4} \alpha_{3,4}\right)\left(\alpha_{1,5} \alpha_{3,5}\right)\left(\alpha_{1,6} \alpha_{3,8}\right)\right. \\
\left(\alpha_{2,6} \alpha_{2,8}\right)\left(\alpha_{3,6} \alpha_{1,8}\right), \\
\left(\alpha_{1,2} \alpha_{3,2}\right)\left(\alpha_{1,3} \alpha_{3,6}\right)\left(\alpha_{2,3} \alpha_{2,6}\right)\left(\alpha_{3,3} \alpha_{1,6}\right)\left(\alpha_{1,4} \alpha_{3,4}\right)\left(\alpha_{1,7} \alpha_{1,8}\right) \\
\left.\left(\alpha_{2,7} \alpha_{2,8}\right)\left(\alpha_{3,7} \alpha_{3,8}\right)\right]
\end{gathered}
$$

with orbits $\left\{\alpha_{1,2}, \alpha_{3,2}\right\},\left\{\alpha_{1,3}, \alpha_{1,7}, \alpha_{3,6}, \alpha_{1,8}\right\},\left\{\alpha_{2,3}, \alpha_{2,7}, \alpha_{2,6}, \alpha_{2,8}\right\}, \quad\left\{\alpha_{3,3}, \alpha_{3,7}, \alpha_{1,6}, \alpha_{3,8}\right\}$, $\left\{\alpha_{1,4}, \alpha_{3,4}\right\},\left\{\alpha_{1,5}, \alpha_{3,5}\right\}$;

$$
H_{3,3}=\left[\left(\alpha_{1,2} \alpha_{1,4}\right)\left(\alpha_{2,2} \alpha_{2,4}\right)\left(\alpha_{3,2} \alpha_{3,4}\right)\left(\alpha_{1,3} \alpha_{3,3}\right)\left(\alpha_{1,6} \alpha_{3,6}\right)\left(\alpha_{1,7} \alpha_{3,8}\right)\right.
$$




$$
\begin{gathered}
\left(\alpha_{2,7} \alpha_{2,8}\right)\left(\alpha_{3,7} \alpha_{1,8}\right) \\
\left(\alpha_{1,2} \alpha_{3,2}\right)\left(\alpha_{1,3} \alpha_{3,6}\right)\left(\alpha_{2,3} \alpha_{2,6}\right)\left(\alpha_{3,3} \alpha_{1,6}\right)\left(\alpha_{1,4} \alpha_{3,4}\right)\left(\alpha_{1,7} \alpha_{1,8}\right) \\
\left.\left(\alpha_{2,7} \alpha_{2,8}\right)\left(\alpha_{3,7} \alpha_{3,8}\right)\right]
\end{gathered}
$$

with orbits $\left\{\alpha_{1,2}, \alpha_{1,4}, \alpha_{3,2}, \alpha_{3,4}\right\}, \quad\left\{\alpha_{2,2}, \alpha_{2,4}\right\}, \quad\left\{\alpha_{1,3}, \alpha_{3,3}, \alpha_{3,6}, \alpha_{1,6}\right\}, \quad\left\{\alpha_{2,3}, \alpha_{2,6}\right\}$, $\left\{\alpha_{1,7}, \alpha_{3,8}, \alpha_{1,8}, \alpha_{3,7}\right\},\left\{\alpha_{2,7}, \alpha_{2,8}\right\}$;

$$
\begin{gathered}
H_{3,4}=\left[\left(\alpha_{1,1} \alpha_{3,1}\right)\left(\alpha_{1,2} \alpha_{1,4}\right)\left(\alpha_{2,2} \alpha_{2,4}\right)\left(\alpha_{3,2} \alpha_{3,4}\right)\left(\alpha_{1,3} \alpha_{3,6}\right)\left(\alpha_{2,3} \alpha_{2,6}\right)\right. \\
\left(\alpha_{3,3} \alpha_{1,6}\right)\left(\alpha_{1,5} \alpha_{3,5}\right) \\
\left(\alpha_{1,2} \alpha_{3,2}\right)\left(\alpha_{1,3} \alpha_{3,6}\right)\left(\alpha_{2,3} \alpha_{2,6}\right)\left(\alpha_{3,3} \alpha_{1,6}\right)\left(\alpha_{1,4} \alpha_{3,4}\right)\left(\alpha_{1,7} \alpha_{1,8}\right) \\
\left.\left(\alpha_{2,7} \alpha_{2,8}\right)\left(\alpha_{3,7} \alpha_{3,8}\right)\right]
\end{gathered}
$$

with orbits $\left\{\alpha_{1,1}, \alpha_{3,1}\right\}, \quad\left\{\alpha_{1,2}, \alpha_{1,4}, \alpha_{3,2}, \alpha_{3,4}\right\}, \quad\left\{\alpha_{2,2}, \alpha_{2,4}\right\}, \quad\left\{\alpha_{1,3}, \alpha_{3,6}\right\}, \quad\left\{\alpha_{2,3}, \alpha_{2,6}\right\}$, $\left\{\alpha_{3,3}, \alpha_{1,6}\right\},\left\{\alpha_{1,5}, \alpha_{3,5}\right\},\left\{\alpha_{1,7}, \alpha_{1,8}\right\},\left\{\alpha_{2,7}, \alpha_{2,8}\right\},\left\{\alpha_{3,7}, \alpha_{3,8}\right\}$.

$\mathbf{n}=\mathbf{2}, H \cong C_{3}(|H|=3, i=1): \operatorname{rk} N_{H}=12$ and $\left(N_{H}\right)^{*} / N_{H} \cong(\mathbb{Z} / 3 \mathbb{Z})^{6}$.

$$
\begin{gathered}
H_{2,1}=\left[\left(\alpha_{1,3} \alpha_{1,4} \alpha_{1,6}\right)\left(\alpha_{2,3} \alpha_{2,4} \alpha_{2,6}\right)\left(\alpha_{3,3} \alpha_{3,4} \alpha_{3,6}\right)\left(\alpha_{1,5} \alpha_{1,8} \alpha_{1,7}\right)\right. \\
\left.\left(\alpha_{2,5} \alpha_{2,8} \alpha_{2,7}\right)\left(\alpha_{3,5} \alpha_{3,8} \alpha_{3,7}\right)\right]
\end{gathered}
$$

with orbits $\left\{\alpha_{1,3}, \alpha_{1,4}, \alpha_{1,6}\right\}, \quad\left\{\alpha_{2,3}, \alpha_{2,4}, \alpha_{2,6}\right\}, \quad\left\{\alpha_{3,3}, \alpha_{3,4}, \alpha_{3,6}\right\}, \quad\left\{\alpha_{1,5}, \alpha_{1,8}, \alpha_{1,7}\right\}$, $\left\{\alpha_{2,5}, \alpha_{2,8}, \alpha_{2,7}\right\},\left\{\alpha_{3,5}, \alpha_{3,8}, \alpha_{3,7}\right\}$.

$\mathbf{n}=\mathbf{1}, H \cong C_{2}(|H|=2, i=1): \operatorname{rk} N_{H}=8$ and $\left(N_{H}\right)^{*} / N_{H} \cong(\mathbb{Z} / 2 \mathbb{Z})^{8}$.

$$
\begin{gathered}
H_{1,1}=\left[\left(\alpha_{1,1} \alpha_{3,1}\right)\left(\alpha_{1,2} \alpha_{3,2}\right)\left(\alpha_{1,3} \alpha_{3,3}\right)\left(\alpha_{1,4} \alpha_{3,4}\right)\left(\alpha_{1,5} \alpha_{3,5}\right)\left(\alpha_{1,6} \alpha_{3,6}\right)\right. \\
\left.\left(\alpha_{1,7} \alpha_{3,7}\right)\left(\alpha_{1,8} \alpha_{3,8}\right)\right]
\end{gathered}
$$

with orbits $\left\{\alpha_{1,1}, \alpha_{3,1}\right\}, \quad\left\{\alpha_{1,2}, \alpha_{3,2}\right\}, \quad\left\{\alpha_{1,3}, \alpha_{3,3}\right\}, \quad\left\{\alpha_{1,4}, \alpha_{3,4}\right\}, \quad\left\{\alpha_{1,5}, \alpha_{3,5}\right\}, \quad\left\{\alpha_{1,6}, \alpha_{3,6}\right\}$, $\left\{\alpha_{1,7}, \alpha_{3,7}\right\},\left\{\alpha_{1,8}, \alpha_{3,8}\right\}$;

$$
\begin{gathered}
H_{1,2}=\left[\left(\alpha_{1,2} \alpha_{3,2}\right)\left(\alpha_{1,3} \alpha_{3,6}\right)\left(\alpha_{2,3} \alpha_{2,6}\right)\left(\alpha_{3,3} \alpha_{1,6}\right)\left(\alpha_{1,4} \alpha_{3,4}\right)\left(\alpha_{1,7} \alpha_{1,8}\right)\right. \\
\left.\left(\alpha_{2,7} \alpha_{2,8}\right)\left(\alpha_{3,7} \alpha_{3,8}\right)\right]
\end{gathered}
$$

with orbits $\left\{\alpha_{1,2}, \alpha_{3,2}\right\}, \quad\left\{\alpha_{1,3}, \alpha_{3,6}\right\}, \quad\left\{\alpha_{2,3}, \alpha_{2,6}\right\}, \quad\left\{\alpha_{3,3}, \alpha_{1,6}\right\}, \quad\left\{\alpha_{1,4}, \alpha_{3,4}\right\}, \quad\left\{\alpha_{1,7}, \alpha_{1,8}\right\}$, $\left\{\alpha_{2,7}, \alpha_{2,8}\right\},\left\{\alpha_{3,7}, \alpha_{3,8}\right\}$. 
In [17] and [18] these conjugacy classes were described by the direct considerations. Here is the correspondence between these two descriptions: $H_{1,1} \cong(\mathrm{I} .10) ; H_{1,2} \cong$ (II.11) and (II, $\left.11^{\prime}\right) ; H_{2,1} \cong\left(\right.$ II.6); $H_{3,1} \cong$ (I.9); $H_{3,2} \cong$ (II.8), (II.8') and (II.8'); $H_{3,3} \cong$ (II.9); $H_{3,4} \cong\left(\mathrm{II} .9^{\prime}\right) ; H_{4,1} \cong(\mathrm{II} .10)$ and $\left(\mathrm{II} .10^{\prime}\right) ; H_{6,1} \cong(\mathrm{II} .5)$ and $\left(\mathrm{II} .5^{\prime}\right) ; H_{7,1} \cong$ (I.4); $H_{8,1} \cong$ (II.2a); $H_{9,1} \cong$ (I.6); $H_{9,2} \cong\left(\right.$ I.7); $H_{10,1} \cong$ (II.7) and (II.7'"'); $H_{10,2} \cong$ (II.7') and (II.7'); $H_{11,1} \cong(\mathrm{I} .8) ; H_{17,1} \cong(\mathrm{II} .4) ; H_{17,2} \cong(\mathrm{IV} .2)$ and $\left(\mathrm{IV} .2^{\prime}\right) ; H_{18,1} \cong(\mathrm{I} .3) ; H_{22,1} \cong$ (I.5); $H_{33,1} \cong(\mathrm{II} .2) ; H_{34,1} \cong(\mathrm{II} .3)$ and (II..'); $H_{34,2} \cong\left(\mathrm{IV} .1^{\prime}\right) ; H_{34,3} \cong(\mathrm{IV} .1) ; H_{35,1} \cong$ (I.2); $H_{35,2} \cong(\mathrm{III} .2) ; H_{51,1} \cong(\mathrm{I} .1) ; H_{51,2} \cong(\mathrm{III} .1) ; H_{74,1} \cong(\mathrm{II} .1)$.

Case 20. For the Niemeier lattice

$$
\begin{gathered}
N=N_{20}=N\left(6 A_{4}\right)=\left[6 A_{4},[1(01441)]\right]= \\
{\left[6 A_{4}, \varepsilon_{1,1}+\varepsilon_{1,3}-\varepsilon_{1,4}-\varepsilon_{1,5}+\varepsilon_{1,6}, \varepsilon_{1,1}+\varepsilon_{1,2}+\varepsilon_{1,4}-\varepsilon_{1,5}-\varepsilon_{1,6}, \varepsilon_{1,1}-\varepsilon_{1,2}+\varepsilon_{1,3}+\varepsilon_{1,5}-\varepsilon_{1,6},\right.} \\
\left.\varepsilon_{1,1}-\varepsilon_{1,2}-\varepsilon_{1,3}+\varepsilon_{1,4}+\varepsilon_{1,6}, \varepsilon_{1,1}+\varepsilon_{1,2}-\varepsilon_{1,3}-\varepsilon_{1,4}+\varepsilon_{1,5}\right]
\end{gathered}
$$

the group $A=A\left(N_{20}\right)$ has the order 240 , and it is generated by

$$
\begin{gathered}
\widetilde{(12)}=\left(\alpha_{1,1} \alpha_{1,5} \alpha_{4,1} \alpha_{4,5}\right)\left(\alpha_{2,1} \alpha_{2,5} \alpha_{3,1} \alpha_{3,5}\right) \\
\left(\alpha_{1,2} \alpha_{4,6} \alpha_{4,2} \alpha_{1,6}\right)\left(\alpha_{2,2} \alpha_{3,6} \alpha_{3,2} \alpha_{2,6}\right)\left(\alpha_{1,3} \alpha_{4,4} \alpha_{4,3} \alpha_{1,4}\right)\left(\alpha_{2,3} \alpha_{3,4} \alpha_{3,3} \alpha_{2,4}\right), \\
\widetilde{(23)}=\left(\alpha_{1,1} \alpha_{1,6} \alpha_{4,1} \alpha_{4,6}\right)\left(\alpha_{2,1} \alpha_{2,6} \alpha_{3,1} \alpha_{3,6}\right) \\
\left(\alpha_{1,2} \alpha_{1,3} \alpha_{4,2} \alpha_{4,3}\right)\left(\alpha_{2,2} \alpha_{2,3} \alpha_{3,2} \alpha_{3,3}\right)\left(\alpha_{1,4} \alpha_{4,5} \alpha_{4,4} \alpha_{1,5}\right)\left(\alpha_{2,4} \alpha_{3,5} \alpha_{3,4} \alpha_{2,5}\right), \\
\widetilde{(34)}=\left(\alpha_{1,1} \alpha_{1,2} \alpha_{4,1} \alpha_{4,2}\right)\left(\alpha_{2,1} \alpha_{2,2} \alpha_{3,1} \alpha_{3,2}\right) \\
\left(\alpha_{1,3} \alpha_{1,4} \alpha_{4,3} \alpha_{4,4}\right)\left(\alpha_{2,3} \alpha_{2,4} \alpha_{3,3} \alpha_{3,4}\right)\left(\alpha_{1,5} \alpha_{4,6} \alpha_{4,5} \alpha_{1,6}\right)\left(\alpha_{2,5} \alpha_{3,6} \alpha_{3,5} \alpha_{2,6}\right), \\
\widetilde{(45)}=\left(\alpha_{1,1} \alpha_{1,3} \alpha_{4,1} \alpha_{4,3}\right)\left(\alpha_{2,1} \alpha_{2,3} \alpha_{3,1} \alpha_{3,3}\right) \\
\left(\alpha_{1,2} \alpha_{1,6} \alpha_{4,2} \alpha_{4,6}\right)\left(\alpha_{2,2} \alpha_{2,6} \alpha_{3,2} \alpha_{3,6}\right)\left(\alpha_{1,4} \alpha_{1,5} \alpha_{4,4} \alpha_{4,5}\right)\left(\alpha_{2,4} \alpha_{2,5} \alpha_{3,4} \alpha_{3,5}\right) .
\end{gathered}
$$

(see [5, Chs. 16, 18] and [17], [18]).

\section{Classification of KahK3 conjugacy classes for $A\left(N_{20}\right)$ :}

$$
\begin{gathered}
\mathbf{n}=\mathbf{3 2}, H \cong \operatorname{Hol}\left(C_{5}\right)(|H|=20, i=3): \operatorname{rk} N_{H}=18 \text { and }\left(N_{H}\right)^{*} / N_{H} \cong(\mathbb{Z} / 10 \mathbb{Z})^{2} \times \mathbb{Z} / 5 \mathbb{Z} . \\
H_{32,1}=\left[\left(\alpha_{1,2} \alpha_{4,2}\right)\left(\alpha_{2,2} \alpha_{3,2}\right)\left(\alpha_{1,3} \alpha_{4,4} \alpha_{1,6} \alpha_{4,5}\right)\left(\alpha_{2,3} \alpha_{3,4} \alpha_{2,6} \alpha_{3,5}\right)\right.
\end{gathered}
$$




$$
\begin{gathered}
\left(\alpha_{3,3} \alpha_{2,4} \alpha_{3,6} \alpha_{2,5}\right)\left(\alpha_{4,3} \alpha_{1,4} \alpha_{4,6} \alpha_{1,5}\right) \\
\left(\alpha_{1,2} \alpha_{1,3} \alpha_{1,4} \alpha_{1,5} \alpha_{1,6}\right)\left(\alpha_{2,2} \alpha_{2,3} \alpha_{2,4} \alpha_{2,5} \alpha_{2,6}\right)\left(\alpha_{3,2} \alpha_{3,3} \alpha_{3,4} \alpha_{3,5} \alpha_{3,6}\right) \\
\left.\left(\alpha_{4,2} \alpha_{4,3} \alpha_{4,4} \alpha_{4,5} \alpha_{4,6}\right)\right]
\end{gathered}
$$

with orbits $\quad\left\{\alpha_{1,2}, \alpha_{4,2}, \alpha_{1,3}, \alpha_{4,3}, \alpha_{4,4}, \alpha_{1,4}, \alpha_{1,6}, \alpha_{4,5}, \alpha_{4,6}, \alpha_{1,5}\right\}, \quad\left\{\alpha_{2,2}, \alpha_{3,2}, \alpha_{2,3}, \alpha_{3,3}\right.$, $\left.\alpha_{3,4}, \alpha_{2,4}, \alpha_{2,6}, \alpha_{3,5}, \alpha_{3,6}, \alpha_{2,5}\right\}$;

$$
\begin{gathered}
H_{32,2}=\left[\left(\alpha_{1,1} \alpha_{4,1}\right)\left(\alpha_{2,1} \alpha_{3,1}\right)\left(\alpha_{1,3} \alpha_{1,4} \alpha_{1,6} \alpha_{1,5}\right)\left(\alpha_{2,3} \alpha_{2,4} \alpha_{2,6} \alpha_{2,5}\right)\right. \\
\left(\alpha_{3,3} \alpha_{3,4} \alpha_{3,6} \alpha_{3,5}\right)\left(\alpha_{4,3} \alpha_{4,4} \alpha_{4,6} \alpha_{4,5}\right), \\
\left(\alpha_{1,2} \alpha_{1,3} \alpha_{1,4} \alpha_{1,5} \alpha_{1,6}\right)\left(\alpha_{2,2} \alpha_{2,3} \alpha_{2,4} \alpha_{2,5} \alpha_{2,6}\right)\left(\alpha_{3,2} \alpha_{3,3} \alpha_{3,4} \alpha_{3,5} \alpha_{3,6}\right) \\
\left.\left(\alpha_{4,2} \alpha_{4,3} \alpha_{4,4} \alpha_{4,5} \alpha_{4,6}\right)\right]
\end{gathered}
$$

with orbits $\left\{\alpha_{1,1}, \alpha_{4,1}\right\},\left\{\alpha_{2,1}, \alpha_{3,1}\right\}, \quad\left\{\alpha_{1,2}, \alpha_{1,3}, \alpha_{1,4}, \alpha_{1,6}, \alpha_{1,5}\right\}, \quad\left\{\alpha_{2,2}, \alpha_{2,3}, \alpha_{2,4}, \alpha_{2,6}, \alpha_{2,5}\right\}$, $\left\{\alpha_{3,2}, \alpha_{3,3}, \alpha_{3,4}, \alpha_{3,6}, \alpha_{3,5}\right\},\left\{\alpha_{4,2}, \alpha_{4,3}, \alpha_{4,4}, \alpha_{4,6}, \alpha_{4,5}\right\}$.

$\mathbf{n}=\mathbf{1 6}, H \cong D_{10}(|H|=10, i=1): \operatorname{rk} N_{H}=16$ and $\left(N_{H}\right)^{*} / N_{H} \cong(\mathbb{Z} / 5 \mathbb{Z})^{4}$.

$$
\begin{gathered}
H_{16,1}=\left[\left(\alpha_{1,3} \alpha_{1,6}\right)\left(\alpha_{2,3} \alpha_{2,6}\right)\left(\alpha_{3,3} \alpha_{3,6}\right)\left(\alpha_{4,3} \alpha_{4,6}\right)\left(\alpha_{1,4} \alpha_{1,5}\right)\left(\alpha_{2,4} \alpha_{2,5}\right)\right. \\
\left(\alpha_{3,4} \alpha_{3,5}\right)\left(\alpha_{4,4} \alpha_{4,5}\right), \\
\left(\alpha_{1,2} \alpha_{1,3} \alpha_{1,4} \alpha_{1,5} \alpha_{1,6}\right)\left(\alpha_{2,2} \alpha_{2,3} \alpha_{2,4} \alpha_{2,5} \alpha_{2,6}\right)\left(\alpha_{3,2} \alpha_{3,3} \alpha_{3,4} \alpha_{3,5} \alpha_{3,6}\right) \\
\left.\left(\alpha_{4,2} \alpha_{4,3} \alpha_{4,4} \alpha_{4,5} \alpha_{4,6}\right)\right]
\end{gathered}
$$

with orbits $\left\{\alpha_{1,2}, \alpha_{1,3}, \alpha_{1,6}, \alpha_{1,4}, \alpha_{1,5}\right\},\left\{\alpha_{2,2}, \alpha_{2,3}, \alpha_{2,6}, \alpha_{2,4}, \alpha_{2,5}\right\},\left\{\alpha_{3,2}, \alpha_{3,3}, \alpha_{3,6}, \alpha_{3,4}, \alpha_{3,5}\right\}$, $\left\{\alpha_{4,2}, \alpha_{4,3}, \alpha_{4,6}, \alpha_{4,4}, \alpha_{4,5}\right\}$.

$$
\begin{gathered}
\mathbf{n}=\mathbf{5}, H \cong C_{5}(|H|=5, i=1): \\
H_{5,1}=\left[\left(\alpha_{1,2} \alpha_{1,3} \alpha_{1,4} \alpha_{1,5} \alpha_{1,6}\right)\left(\alpha_{2,2} \alpha_{2,3} \alpha_{2,4} \alpha_{2,5} \alpha_{2,6}\right)\left(\alpha_{3,2} \alpha_{3,3} \alpha_{3,4} \alpha_{3,5} \alpha_{3,6}\right)\right. \\
\left.\left(\alpha_{4,2} \alpha_{4,3} \alpha_{4,4} \alpha_{4,5} \alpha_{4,6}\right)\right]
\end{gathered}
$$

with $C l o s\left(H_{5,1}\right)=H_{16,1}$ above.

$$
\begin{aligned}
& \mathbf{n}=4, H \cong C_{4}(|H|=4, i=1): \operatorname{rk} N_{H}=14 \text { and }\left(N_{H}\right)^{*} / N_{H} \cong(\mathbb{Z} / 4 \mathbb{Z})^{4} \times(\mathbb{Z} / 2 \mathbb{Z})^{2} \text {. } \\
& H_{4,1}=\left[\left(\alpha_{1,2} \alpha_{4,2}\right)\left(\alpha_{2,2} \alpha_{3,2}\right)\left(\alpha_{1,3} \alpha_{4,4} \alpha_{1,6} \alpha_{4,5}\right)\left(\alpha_{2,3} \alpha_{3,4} \alpha_{2,6} \alpha_{3,5}\right)\right. \\
& \left.\left(\alpha_{3,3} \alpha_{2,4} \alpha_{3,6} \alpha_{2,5}\right)\left(\alpha_{4,3} \alpha_{1,4} \alpha_{4,6} \alpha_{1,5}\right)\right]
\end{aligned}
$$


with orbits $\quad\left\{\alpha_{1,2}, \alpha_{4,2}\right\}, \quad\left\{\alpha_{2,2}, \alpha_{3,2}\right\}, \quad\left\{\alpha_{1,3}, \alpha_{4,4}, \alpha_{1,6}, \alpha_{4,5}\right\}, \quad\left\{\alpha_{2,3}, \alpha_{3,4}, \alpha_{2,6}, \alpha_{3,5}\right\}$, $\left\{\alpha_{3,3}, \alpha_{2,4}, \alpha_{3,6}, \alpha_{2,5}\right\},\left\{\alpha_{4,3}, \alpha_{1,4}, \alpha_{4,6}, \alpha_{1,5}\right\}$.

$\mathbf{n}=\mathbf{1}, H \cong C_{2}(|H|=2, i=1): \operatorname{rk} N_{H}=8$ and $\left(N_{H}\right)^{*} / N_{H} \cong(\mathbb{Z} / 2 \mathbb{Z})^{8}$.

$$
\begin{gathered}
H_{1,1}=\left[\left(\alpha_{1,3} \alpha_{1,6}\right)\left(\alpha_{2,3} \alpha_{2,6}\right)\left(\alpha_{3,3} \alpha_{3,6}\right)\left(\alpha_{4,3} \alpha_{4,6}\right)\left(\alpha_{1,4} \alpha_{1,5}\right)\left(\alpha_{2,4} \alpha_{2,5}\right)\right. \\
\left.\left(\alpha_{3,4} \alpha_{3,5}\right)\left(\alpha_{4,4} \alpha_{4,5}\right)\right]
\end{gathered}
$$

with orbits $\left\{\alpha_{1,3}, \alpha_{1,6}\right\}, \quad\left\{\alpha_{2,3}, \alpha_{2,6}\right\}, \quad\left\{\alpha_{3,3}, \alpha_{3,6}\right\}, \quad\left\{\alpha_{4,3}, \alpha_{4,6}\right\}, \quad\left\{\alpha_{1,4}, \alpha_{1,5}\right\}, \quad\left\{\alpha_{2,4}, \alpha_{2,5}\right\}$, $\left\{\alpha_{3,4}, \alpha_{3,5}\right\},\left\{\alpha_{4,4}, \alpha_{4,5}\right\}$.

In [17] and [18], these conjugacy classes were described by the direct considerations.

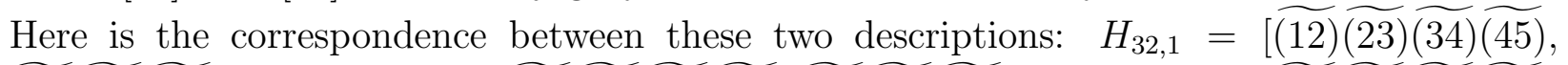
$\widetilde{(45)} \widetilde{(23)} \widetilde{(34)} \varphi]$ in 1$) ; H_{32,2}=[\widetilde{(12)} \widetilde{(23)} \widetilde{(34)} \widetilde{(45)}, \widetilde{(45)(23)(34)}]$ in 1$) ; H_{16,1}=[\widetilde{(12)(23)(34)(45)}$,

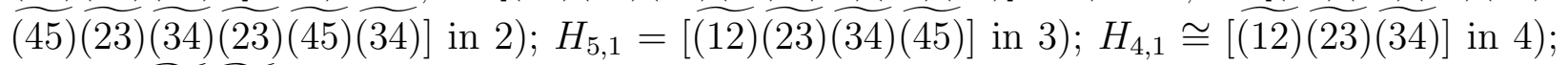
$H_{1,1} \cong[\overline{(12)} \widetilde{(34)}]$ in 5$)$.

Case 19. For the Niemeier lattice

$$
\begin{gathered}
N=N_{19}=N\left(6 D_{4}\right)= \\
{\left[6 D_{4}, \varepsilon_{1,3}+\varepsilon_{1,4}+\varepsilon_{1,5}+\varepsilon_{1,6}, \varepsilon_{1,2}+\varepsilon_{1,4}+\varepsilon_{2,5}+\varepsilon_{3,6}, \varepsilon_{1,1}+\varepsilon_{1,4}+\varepsilon_{3,5}+\varepsilon_{2,6},\right.} \\
\left.\varepsilon_{2,3}+\varepsilon_{2,4}+\varepsilon_{2,5}+\varepsilon_{2,6}, \varepsilon_{2,2}+\varepsilon_{2,4}+\varepsilon_{3,5}+\varepsilon_{1,6}, \varepsilon_{2,1}+\varepsilon_{2,4}+\varepsilon_{1,5}+\varepsilon_{3,6}\right]
\end{gathered}
$$

the group $A=A(N)$ has order 2160 and it is generated by

$$
\begin{gathered}
\varphi=\left(\alpha_{1,1} \alpha_{3,1} \alpha_{4,1}\right)\left(\alpha_{1,2} \alpha_{3,2} \alpha_{4,2}\right)\left(\alpha_{1,3} \alpha_{3,3} \alpha_{4,3}\right)\left(\alpha_{1,4} \alpha_{3,4} \alpha_{4,4}\right) \\
\left(\alpha_{1,5} \alpha_{3,5} \alpha_{4,5}\right)\left(\alpha_{1,6} \alpha_{3,6} \alpha_{4,6}\right), \\
\widetilde{(12)}=\left(\alpha_{4,1} \alpha_{4,2}\right)\left(\alpha_{1,1} \alpha_{3,2}\right)\left(\alpha_{3,1} \alpha_{1,2}\right)\left(\alpha_{1,3} \alpha_{3,3}\right)\left(\alpha_{1,4} \alpha_{3,4}\right)\left(\alpha_{1,5} \alpha_{3,5}\right) \\
\left(\alpha_{1,6} \alpha_{3,6}\right)\left(\alpha_{2,1} \alpha_{2,2}\right), \\
\widetilde{(23)}=\left(\alpha_{1,1} \alpha_{3,1}\right)\left(\alpha_{1,2} \alpha_{3,3}\right)\left(\alpha_{3,2} \alpha_{1,3}\right)\left(\alpha_{4,2} \alpha_{4,3}\right)\left(\alpha_{1,4} \alpha_{3,4}\right)\left(\alpha_{1,5} \alpha_{4,5}\right) \\
\left(\alpha_{3,6} \alpha_{4,6}\right)\left(\alpha_{2,2} \alpha_{2,3}\right), \\
\widetilde{(34)}=\left(\alpha_{1,1} \alpha_{3,1}\right)\left(\alpha_{1,2} \alpha_{3,2}\right)\left(\alpha_{4,3} \alpha_{4,4}\right)\left(\alpha_{1,3} \alpha_{3,4}\right)\left(\alpha_{3,3} \alpha_{1,4}\right)\left(\alpha_{1,5} \alpha_{3,5}\right) \\
\left(\alpha_{1,6} \alpha_{3,6}\right)\left(\alpha_{2,3} \alpha_{2,4}\right),
\end{gathered}
$$




$$
\begin{gathered}
\left(\alpha_{1,6} \alpha_{3,6}\right)\left(\alpha_{2,4} \alpha_{2,5}\right), \\
\widetilde{(56)}=\left(\alpha_{1,1} \alpha_{3,1}\right)\left(\alpha_{1,2} \alpha_{3,2}\right)\left(\alpha_{1,3} \alpha_{3,3}\right)\left(\alpha_{1,4} \alpha_{3,4}\right)\left(\alpha_{1,5} \alpha_{3,6}\right)\left(\alpha_{3,5} \alpha_{1,6}\right) \\
\left(\alpha_{4,5} \alpha_{4,6}\right)\left(\alpha_{2,5} \alpha_{2,6}\right)
\end{gathered}
$$

(see [5, Chs. 16, 18]) and [17], [18]).

\section{Classification of KahK3 conjugacy classes for $A\left(N_{19}\right)$ :}

$\mathbf{n}=\mathbf{7 0}, H \cong \mathfrak{S}_{5}(|H|=120, i=34): \operatorname{rk} N_{H}=19$ and $\left(N_{H}\right)^{*} / N_{H} \cong \mathbb{Z} / 60 \mathbb{Z} \times \mathbb{Z} / 5 \mathbb{Z}$.

$$
\begin{gathered}
H_{70,1}=\left[\left(\alpha_{1,1} \alpha_{1,2} \alpha_{3,3} \alpha_{4,5} \alpha_{3,4}\right)\left(\alpha_{2,1} \alpha_{2,2} \alpha_{2,3} \alpha_{2,5} \alpha_{2,4}\right)\right. \\
\left(\alpha_{3,1} \alpha_{3,2} \alpha_{4,3} \alpha_{1,5} \alpha_{4,4}\right)\left(\alpha_{4,1} \alpha_{4,2} \alpha_{1,3} \alpha_{3,5} \alpha_{1,4}\right) \\
\left(\alpha_{1,1} \alpha_{4,2}\right)\left(\alpha_{2,1} \alpha_{2,2}\right)\left(\alpha_{3,1} \alpha_{3,2}\right)\left(\alpha_{4,1} \alpha_{1,2}\right)\left(\alpha_{1,3} \alpha_{4,3}\right)\left(\alpha_{1,4} \alpha_{4,4}\right) \\
\left.\left(\alpha_{1,5} \alpha_{4,5}\right)\left(\alpha_{1,6} \alpha_{4,6}\right)\right]
\end{gathered}
$$

with $\quad$ orbits $\left\{\alpha_{1,1}, \alpha_{1,2}, \alpha_{4,2}, \alpha_{3,3}, \alpha_{4,1}, \alpha_{1,3}, \alpha_{4,5}, \alpha_{3,5}, \alpha_{4,3}, \alpha_{3,4}, \alpha_{1,5}, \alpha_{1,4}, \alpha_{4,4}, \alpha_{3,1}, \alpha_{3,2}\right\}$, $\left\{\alpha_{2,1}, \alpha_{2,2}, \alpha_{2,3}, \alpha_{2,5}, \alpha_{2,4}\right\},\left\{\alpha_{1,6}, \alpha_{4,6}\right\}$.

$\mathbf{n}=\mathbf{6 1}, H \cong \mathfrak{A}_{4,3}(|H|=72, i=43): \operatorname{rk} N_{H}=18$ and $\left(N_{H}\right)^{*} / N_{H} \cong(\mathbb{Z} / 12 \mathbb{Z})^{2} \times \mathbb{Z} / 3 \mathbb{Z}$.

$$
\begin{gathered}
H_{61,1}=\left[\left(\alpha_{1,1} \alpha_{1,3}\right)\left(\alpha_{2,1} \alpha_{2,3}\right)\left(\alpha_{3,1} \alpha_{4,3}\right)\left(\alpha_{4,1} \alpha_{3,3}\right)\left(\alpha_{3,2} \alpha_{4,2}\right)\left(\alpha_{3,4} \alpha_{4,4}\right)\right. \\
\left(\alpha_{1,5} \alpha_{4,5}\right)\left(\alpha_{1,6} \alpha_{3,6}\right), \\
\left(\alpha_{1,2} \alpha_{1,3} \alpha_{1,4}\right)\left(\alpha_{2,2} \alpha_{2,3} \alpha_{2,4}\right)\left(\alpha_{3,2} \alpha_{3,3} \alpha_{3,4}\right)\left(\alpha_{4,2} \alpha_{4,3} \alpha_{4,4}\right) \\
\left(\alpha_{1,5} \alpha_{3,5} \alpha_{4,5}\right)\left(\alpha_{1,6} \alpha_{4,6} \alpha_{3,6}\right), \\
\left(\alpha_{1,1} \alpha_{4,2}\right)\left(\alpha_{2,1} \alpha_{2,2}\right)\left(\alpha_{3,1} \alpha_{3,2}\right)\left(\alpha_{4,1} \alpha_{1,2}\right)\left(\alpha_{1,3} \alpha_{4,3}\right)\left(\alpha_{1,4} \alpha_{4,4}\right) \\
\left.\left(\alpha_{1,5} \alpha_{4,5}\right)\left(\alpha_{1,6} \alpha_{4,6}\right)\right]
\end{gathered}
$$

with orbits $\left\{\alpha_{1,1}, \alpha_{1,3}, \alpha_{4,2}, \alpha_{1,4}, \alpha_{4,3}, \alpha_{3,2}, \alpha_{1,2}, \alpha_{4,4}, \alpha_{3,1}, \alpha_{3,3}, \alpha_{4,1}, \alpha_{3,4}\right\},\left\{\alpha_{2,1}, \alpha_{2,3}, \alpha_{2,2}, \alpha_{2,4}\right\}$, $\left\{\alpha_{1,5}, \alpha_{4,5}, \alpha_{3,5}\right\},\left\{\alpha_{1,6}, \alpha_{3,6}, \alpha_{4,6}\right\}$.

$\mathbf{n}=\mathbf{5 5}, H \cong \mathfrak{A}_{5}(|H|=60, i=5): \operatorname{rk} N_{H}=18$ and $\left(N_{H}\right)^{*} / N_{H} \cong \mathbb{Z} / 30 \mathbb{Z} \times \mathbb{Z} / 10 \mathbb{Z}$.

$$
\begin{gathered}
H_{55,1}=\left[\left(\alpha_{1,1} \alpha_{1,3} \alpha_{1,6} \alpha_{4,2} \alpha_{4,5}\right)\left(\alpha_{2,1} \alpha_{2,3} \alpha_{2,6} \alpha_{2,2} \alpha_{2,5}\right)\right. \\
\left(\alpha_{3,1} \alpha_{3,3} \alpha_{3,6} \alpha_{1,2} \alpha_{1,5}\right)\left(\alpha_{4,1} \alpha_{4,3} \alpha_{4,6} \alpha_{3,2} \alpha_{3,5}\right) \\
\left(\alpha_{1,1} \alpha_{1,2}\right)\left(\alpha_{2,1} \alpha_{2,2}\right)\left(\alpha_{3,1} \alpha_{3,2}\right)\left(\alpha_{4,1} \alpha_{4,2}\right)\left(\alpha_{1,5} \alpha_{1,6}\right)\left(\alpha_{2,5} \alpha_{2,6}\right) \\
\left.\left(\alpha_{3,5} \alpha_{3,6}\right)\left(\alpha_{4,5} \alpha_{4,6}\right)\right]
\end{gathered}
$$


with $\quad$ orbits $\quad\left\{\alpha_{1,1}, \alpha_{1,3}, \alpha_{1,2}, \alpha_{1,6}, \alpha_{1,5}, \alpha_{4,2}, \alpha_{3,1}, \alpha_{4,5}, \alpha_{4,1}, \alpha_{3,3}, \alpha_{3,2}, \alpha_{4,6}, \alpha_{4,3}, \alpha_{3,6}, \alpha_{3,5}\right\}$, $\left\{\alpha_{2,1}, \alpha_{2,3}, \alpha_{2,2}, \alpha_{2,6}, \alpha_{2,5}\right\}$.

$\mathbf{n}=\mathbf{4 8}, H \cong \mathfrak{S}_{3,3}(|H|=36, i=10): \operatorname{rk} N_{H}=18,\left(N_{H}\right)^{*} / N_{H} \cong \mathbb{Z} / 18 \mathbb{Z} \times \mathbb{Z} / 6 \mathbb{Z} \times(\mathbb{Z} / 3 \mathbb{Z})^{2}$.

$$
\begin{gathered}
H_{48,1}=\left[\left(\alpha_{3,1} \alpha_{4,1}\right)\left(\alpha_{1,2} \alpha_{3,2}\right)\left(\alpha_{1,3} \alpha_{4,3}\right)\left(\alpha_{1,4} \alpha_{4,5}\right)\left(\alpha_{2,4} \alpha_{2,5}\right)\left(\alpha_{3,4} \alpha_{3,5}\right)\right. \\
\left(\alpha_{4,4} \alpha_{1,5}\right)\left(\alpha_{1,6} \alpha_{4,6}\right), \\
\left(\alpha_{1,1} \alpha_{4,2} \alpha_{3,1} \alpha_{1,2} \alpha_{4,1} \alpha_{3,2}\right)\left(\alpha_{2,1} \alpha_{2,2}\right)\left(\alpha_{1,3} \alpha_{4,3} \alpha_{3,3}\right)\left(\alpha_{1,4} \alpha_{4,4} \alpha_{3,4}\right) \\
\left.\left(\alpha_{1,5} \alpha_{4,6} \alpha_{3,5} \alpha_{1,6} \alpha_{4,5} \alpha_{3,6}\right)\left(\alpha_{2,5} \alpha_{2,6}\right)\right]
\end{gathered}
$$

with orbits $\quad\left\{\alpha_{1,1}, \alpha_{4,2}, \alpha_{3,1}, \alpha_{4,1}, \alpha_{1,2}, \alpha_{3,2}\right\}, \quad\left\{\alpha_{2,1}, \alpha_{2,2}\right\}, \quad\left\{\alpha_{1,3}, \alpha_{4,3}, \alpha_{3,3}\right\}$, $\left\{\alpha_{1,4}, \alpha_{4,5}, \alpha_{4,4}, \alpha_{3,6}, \alpha_{1,5}, \alpha_{3,4}, \alpha_{4,6}, \alpha_{3,5}, \alpha_{1,6}\right\},\left\{\alpha_{2,4}, \alpha_{2,5}, \alpha_{2,6}\right\}$.

$\mathbf{n}=\mathbf{4 7}, H \cong C_{3} \times \mathfrak{A}_{4}(|H|=36, i=11)$ :

$$
\begin{gathered}
H_{47,1}=\left[\left(\alpha_{1,2} \alpha_{1,3} \alpha_{1,4}\right)\left(\alpha_{2,2} \alpha_{2,3} \alpha_{2,4}\right)\left(\alpha_{3,2} \alpha_{3,3} \alpha_{3,4}\right)\left(\alpha_{4,2} \alpha_{4,3} \alpha_{4,4}\right)\right. \\
\left(\alpha_{1,5} \alpha_{3,5} \alpha_{4,5}\right)\left(\alpha_{1,6} \alpha_{4,6} \alpha_{3,6}\right), \\
\left(\alpha_{1,1} \alpha_{3,2} \alpha_{4,1} \alpha_{1,2} \alpha_{3,1} \alpha_{4,2}\right)\left(\alpha_{2,1} \alpha_{2,2}\right)\left(\alpha_{1,3} \alpha_{3,4} \alpha_{4,3} \alpha_{1,4} \alpha_{3,3} \alpha_{4,4}\right)\left(\alpha_{2,3} \alpha_{2,4}\right) \\
\left.\left(\alpha_{1,5} \alpha_{3,5} \alpha_{4,5}\right)\left(\alpha_{1,6} \alpha_{3,6} \alpha_{4,6}\right)\right]
\end{gathered}
$$

with $\operatorname{Clos}\left(H_{47,1}\right)=H_{61,1}$ above.

$\mathbf{n}=\mathbf{3 6}, H \cong C_{3} \rtimes D_{8}(|H|=24, i=8):$

$$
\begin{gathered}
H_{36,1}=\left[\left(\alpha_{3,1} \alpha_{4,1}\right)\left(\alpha_{1,2} \alpha_{1,3}\right)\left(\alpha_{2,2} \alpha_{2,3}\right)\left(\alpha_{3,2} \alpha_{4,3}\right)\left(\alpha_{4,2} \alpha_{3,3}\right)\left(\alpha_{3,4} \alpha_{4,4}\right)\right. \\
\left(\alpha_{1,5} \alpha_{3,5}\right)\left(\alpha_{1,6} \alpha_{4,6}\right), \\
\left(\alpha_{1,1} \alpha_{3,2} \alpha_{4,1} \alpha_{1,2} \alpha_{3,1} \alpha_{4,2}\right)\left(\alpha_{2,1} \alpha_{2,2}\right)\left(\alpha_{1,3} \alpha_{3,4} \alpha_{4,3} \alpha_{1,4} \alpha_{3,3} \alpha_{4,4}\right)\left(\alpha_{2,3} \alpha_{2,4}\right) \\
\left.\left(\alpha_{1,5} \alpha_{3,5} \alpha_{4,5}\right)\left(\alpha_{1,6} \alpha_{3,6} \alpha_{4,6}\right)\right]
\end{gathered}
$$

with $\operatorname{Clos}\left(H_{36,1}\right)=H_{61,1}$ above.

$\mathbf{n}=\mathbf{3 4}, H \cong \mathfrak{S}_{4}(|H|=24, i=12): \operatorname{rk} N_{H}=17$ and $\left(N_{H}\right)^{*} / N_{H} \cong(\mathbb{Z} / 12 \mathbb{Z})^{2} \times \mathbb{Z} / 4 \mathbb{Z}$.

$$
\begin{gathered}
H_{34,1}=\left[\left(\alpha_{1,2} \alpha_{1,5} \alpha_{1,6}\right)\left(\alpha_{2,2} \alpha_{2,5} \alpha_{2,6}\right)\left(\alpha_{3,2} \alpha_{3,5} \alpha_{3,6}\right)\left(\alpha_{4,2} \alpha_{4,5} \alpha_{4,6}\right)\right. \\
\left(\alpha_{1,3} \alpha_{3,3} \alpha_{4,3}\right)\left(\alpha_{1,4} \alpha_{4,4} \alpha_{3,4}\right) \\
\left(\alpha_{1,1} \alpha_{3,2} \alpha_{1,6} \alpha_{3,5}\right)\left(\alpha_{2,1} \alpha_{2,2} \alpha_{2,6} \alpha_{2,5}\right)\left(\alpha_{3,1} \alpha_{1,2} \alpha_{3,6} \alpha_{1,5}\right)
\end{gathered}
$$




$$
\left.\left(\alpha_{4,1} \alpha_{4,2} \alpha_{4,6} \alpha_{4,5}\right)\left(\alpha_{1,3} \alpha_{4,3}\right)\left(\alpha_{3,4} \alpha_{4,4}\right)\right]
$$

with orbits $\left\{\alpha_{1,1}, \alpha_{3,2}, \alpha_{3,5}, \alpha_{1,6}, \alpha_{3,6}, \alpha_{1,2}, \alpha_{1,5}, \alpha_{3,1}\right\}, \quad\left\{\alpha_{2,1}, \alpha_{2,2}, \alpha_{2,5}, \alpha_{2,6}\right\}, \quad\left\{\alpha_{4,1}, \alpha_{4,2}\right.$, $\left.\alpha_{4,5}, \alpha_{4,6}\right\},\left\{\alpha_{1,3}, \alpha_{3,3}, \alpha_{4,3}\right\},\left\{\alpha_{1,4}, \alpha_{4,4}, \alpha_{3,4}\right\}$;

$$
\begin{gathered}
H_{34,2}=\left[\left(\alpha_{1,1} \alpha_{3,1} \alpha_{4,1}\right)\left(\alpha_{1,2} \alpha_{3,5} \alpha_{4,6}\right)\left(\alpha_{2,2} \alpha_{2,5} \alpha_{2,6}\right)\left(\alpha_{3,2} \alpha_{4,5} \alpha_{1,6}\right)\right. \\
\left(\alpha_{4,2} \alpha_{1,5} \alpha_{3,6}\right)\left(\alpha_{1,3} \alpha_{4,3} \alpha_{3,3}\right), \\
\left(\alpha_{1,1} \alpha_{3,2} \alpha_{1,6} \alpha_{3,5}\right)\left(\alpha_{2,1} \alpha_{2,2} \alpha_{2,6} \alpha_{2,5}\right)\left(\alpha_{3,1} \alpha_{1,2} \alpha_{3,6} \alpha_{1,5}\right) \\
\left.\left(\alpha_{4,1} \alpha_{4,2} \alpha_{4,6} \alpha_{4,5}\right)\left(\alpha_{1,3} \alpha_{4,3}\right)\left(\alpha_{3,4} \alpha_{4,4}\right)\right]
\end{gathered}
$$

with orbits $\left\{\alpha_{1,1}, \alpha_{3,1}, \alpha_{3,2}, \alpha_{4,1}, \alpha_{1,2}, \alpha_{4,5}, \alpha_{1,6}, \alpha_{4,2}, \alpha_{3,5}, \alpha_{3,6}, \alpha_{1,5}, \alpha_{4,6}\right\},\left\{\alpha_{2,1}, \alpha_{2,2}, \alpha_{2,5}, \alpha_{2,6}\right\}$, $\left\{\alpha_{1,3}, \alpha_{4,3}, \alpha_{3,3}\right\},\left\{\alpha_{3,4}, \alpha_{4,4}\right\}$.

$\mathbf{n}=32, H \cong H o l\left(C_{5}\right) \quad(|H|=20, \quad i=3): \operatorname{rk} N_{H}=18$ and $\left(N_{H}\right)^{*} / N_{H}$ $\cong(\mathbb{Z} / 10 \mathbb{Z})^{2} \times \mathbb{Z} / 5 \mathbb{Z}$

$$
\begin{gathered}
H_{32,1}=\left[\left(\alpha_{3,1} \alpha_{4,1}\right)\left(\alpha_{1,2} \alpha_{4,6} \alpha_{1,4} \alpha_{3,5}\right)\left(\alpha_{2,2} \alpha_{2,6} \alpha_{2,4} \alpha_{2,5}\right)\left(\alpha_{3,2} \alpha_{3,6} \alpha_{3,4} \alpha_{1,5}\right)\right. \\
\left(\alpha_{4,2} \alpha_{1,6} \alpha_{4,4} \alpha_{4,5}\right)\left(\alpha_{3,3} \alpha_{4,3}\right) \\
\left(\alpha_{1,1} \alpha_{3,2} \alpha_{3,6} \alpha_{1,5} \alpha_{3,4}\right)\left(\alpha_{2,1} \alpha_{2,2} \alpha_{2,6} \alpha_{2,5} \alpha_{2,4}\right)\left(\alpha_{3,1} \alpha_{4,2} \alpha_{4,6} \alpha_{3,5} \alpha_{4,4}\right) \\
\left.\left(\alpha_{4,1} \alpha_{1,2} \alpha_{1,6} \alpha_{4,5} \alpha_{1,4}\right)\right]
\end{gathered}
$$

with orbits $\left\{\alpha_{1,1}, \alpha_{3,2}, \alpha_{3,6}, \alpha_{3,4}, \alpha_{1,5}\right\}, \quad\left\{\alpha_{2,1}, \alpha_{2,2}, \alpha_{2,6}, \alpha_{2,4}, \alpha_{2,5}\right\}, \quad\left\{\alpha_{3,1}, \alpha_{4,1}, \alpha_{4,2}, \alpha_{1,2}\right.$, $\left.\alpha_{1,6}, \alpha_{4,6}, \alpha_{4,4}, \alpha_{4,5}, \alpha_{1,4}, \alpha_{3,5}\right\},\left\{\alpha_{3,3}, \alpha_{4,3}\right\}$.

$\mathbf{n}=\mathbf{3 1}, H \cong C_{3} \times D_{6}(|H|=18, i=3):$

$$
\begin{gathered}
H_{31,1}=\left[\left(\alpha_{1,1} \alpha_{3,1} \alpha_{4,1}\right)\left(\alpha_{1,2} \alpha_{3,2} \alpha_{4,2}\right)\left(\alpha_{1,3} \alpha_{3,3} \alpha_{4,3}\right)\left(\alpha_{1,4} \alpha_{3,4} \alpha_{4,4}\right)\right. \\
\left(\alpha_{1,5} \alpha_{3,5} \alpha_{4,5}\right)\left(\alpha_{1,6} \alpha_{3,6} \alpha_{4,6}\right) \\
\left(\alpha_{1,1} \alpha_{1,2} \alpha_{3,1} \alpha_{4,2} \alpha_{4,1} \alpha_{3,2}\right)\left(\alpha_{2,1} \alpha_{2,2}\right)\left(\alpha_{1,3} \alpha_{4,3}\right)\left(\alpha_{1,4} \alpha_{4,6} \alpha_{1,5} \alpha_{4,4} \alpha_{1,6} \alpha_{4,5}\right) \\
\left.\left(\alpha_{2,4} \alpha_{2,6} \alpha_{2,5}\right)\left(\alpha_{3,4} \alpha_{3,6} \alpha_{3,5}\right)\right]
\end{gathered}
$$

with $C l o s\left(H_{31,1}\right)=H_{48,1}$ above;

$$
\begin{gathered}
H_{31,2}=\left[\left(\alpha_{1,1} \alpha_{3,1} \alpha_{4,1}\right)\left(\alpha_{1,2} \alpha_{4,2} \alpha_{3,2}\right)\left(\alpha_{1,4} \alpha_{1,5} \alpha_{1,6}\right)\left(\alpha_{2,4} \alpha_{2,5} \alpha_{2,6}\right)\right. \\
\left(\alpha_{3,4} \alpha_{3,5} \alpha_{3,6}\right)\left(\alpha_{4,4} \alpha_{4,5} \alpha_{4,6}\right) \\
\left(\alpha_{1,1} \alpha_{1,2} \alpha_{3,1} \alpha_{3,2} \alpha_{4,1} \alpha_{4,2}\right)\left(\alpha_{2,1} \alpha_{2,2}\right)\left(\alpha_{1,3} \alpha_{4,3} \alpha_{3,3}\right)\left(\alpha_{1,4} \alpha_{4,6} \alpha_{3,4} \alpha_{1,6} \alpha_{4,4} \alpha_{3,6}\right)
\end{gathered}
$$




$$
\left.\left(\alpha_{2,4} \alpha_{2,6}\right)\left(\alpha_{1,5} \alpha_{4,5} \alpha_{3,5}\right)\right]
$$

with $C l o s\left(H_{31,2}\right)=H_{48,1}$ above.

$\mathbf{n}=\mathbf{3 0}, H \cong \mathfrak{A}_{3,3}(|H|=18, i=4): \operatorname{rk} N_{H}=16$ and $\left(N_{H}\right)^{*} / N_{H} \cong \mathbb{Z} / 9 \mathbb{Z} \times(\mathbb{Z} / 3 \mathbb{Z})^{4}$.

$$
\begin{gathered}
H_{30,1}=\left[\left(\alpha_{3,1} \alpha_{4,1}\right)\left(\alpha_{1,2} \alpha_{1,3}\right)\left(\alpha_{2,2} \alpha_{2,3}\right)\left(\alpha_{3,2} \alpha_{4,3}\right)\left(\alpha_{4,2} \alpha_{3,3}\right)\left(\alpha_{3,4} \alpha_{4,4}\right)\right. \\
\left(\alpha_{1,5} \alpha_{3,5}\right)\left(\alpha_{1,6} \alpha_{4,6}\right), \\
\left(\alpha_{1,1} \alpha_{3,1} \alpha_{4,1}\right)\left(\alpha_{1,2} \alpha_{3,2} \alpha_{4,2}\right)\left(\alpha_{1,3} \alpha_{3,3} \alpha_{4,3}\right)\left(\alpha_{1,4} \alpha_{3,4} \alpha_{4,4}\right) \\
\left(\alpha_{1,5} \alpha_{3,5} \alpha_{4,5}\right)\left(\alpha_{1,6} \alpha_{3,6} \alpha_{4,6}\right), \\
\left(\alpha_{1,1} \alpha_{3,2}\right)\left(\alpha_{2,1} \alpha_{2,2}\right)\left(\alpha_{3,1} \alpha_{1,2}\right)\left(\alpha_{4,1} \alpha_{4,2}\right)\left(\alpha_{1,3} \alpha_{3,3}\right)\left(\alpha_{1,4} \alpha_{3,4}\right) \\
\left.\left(\alpha_{1,5} \alpha_{3,5}\right)\left(\alpha_{1,6} \alpha_{3,6}\right)\right]
\end{gathered}
$$

with orbits $\quad\left\{\alpha_{1,1}, \alpha_{3,1}, \alpha_{3,2}, \alpha_{4,1}, \alpha_{1,2}, \alpha_{4,3}, \alpha_{4,2}, \alpha_{1,3}, \alpha_{3,3}\right\}, \quad\left\{\alpha_{2,1}, \alpha_{2,2}, \alpha_{2,3}\right\}$, $\left\{\alpha_{1,4}, \alpha_{3,4}, \alpha_{4,4}\right\},\left\{\alpha_{1,5}, \alpha_{3,5}, \alpha_{4,5}\right\},\left\{\alpha_{1,6}, \alpha_{4,6}, \alpha_{3,6}\right\}$.

$\mathbf{n}=\mathbf{2 0}, H \cong Q_{12}(|H|=12, i=1)$ :

$$
\begin{gathered}
H_{20,1}=\left[\left(\alpha_{1,1} \alpha_{1,3} \alpha_{1,2} \alpha_{1,4}\right)\left(\alpha_{2,1} \alpha_{2,3} \alpha_{2,2} \alpha_{2,4}\right)\left(\alpha_{3,1} \alpha_{4,3} \alpha_{3,2} \alpha_{4,4}\right)\right. \\
\left(\alpha_{4,1} \alpha_{3,3} \alpha_{4,2} \alpha_{3,4}\right)\left(\alpha_{3,5} \alpha_{4,5}\right)\left(\alpha_{3,6} \alpha_{4,6}\right) \\
\left(\alpha_{1,1} \alpha_{3,2} \alpha_{4,1} \alpha_{1,2} \alpha_{3,1} \alpha_{4,2}\right)\left(\alpha_{2,1} \alpha_{2,2}\right)\left(\alpha_{1,3} \alpha_{3,4} \alpha_{4,3} \alpha_{1,4} \alpha_{3,3} \alpha_{4,4}\right)\left(\alpha_{2,3} \alpha_{2,4}\right) \\
\left.\left(\alpha_{1,5} \alpha_{3,5} \alpha_{4,5}\right)\left(\alpha_{1,6} \alpha_{3,6} \alpha_{4,6}\right)\right]
\end{gathered}
$$

with $C l o s\left(H_{20,1}\right)=H_{61,1}$ above.

$\mathbf{n}=\mathbf{1 9}, H \cong C_{2} \times C_{6}(|H|=12, i=5):$

$$
\begin{gathered}
H_{19,1}=\left[\left(\alpha_{1,1} \alpha_{1,3}\right)\left(\alpha_{2,1} \alpha_{2,3}\right)\left(\alpha_{3,1} \alpha_{3,3}\right)\left(\alpha_{4,1} \alpha_{4,3}\right)\left(\alpha_{1,2} \alpha_{1,4}\right)\left(\alpha_{2,2} \alpha_{2,4}\right)\right. \\
\left(\alpha_{3,2} \alpha_{3,4}\right)\left(\alpha_{4,2} \alpha_{4,4}\right) \\
\left(\alpha_{1,1} \alpha_{3,2} \alpha_{4,1} \alpha_{1,2} \alpha_{3,1} \alpha_{4,2}\right)\left(\alpha_{2,1} \alpha_{2,2}\right)\left(\alpha_{1,3} \alpha_{3,4} \alpha_{4,3} \alpha_{1,4} \alpha_{3,3} \alpha_{4,4}\right)\left(\alpha_{2,3} \alpha_{2,4}\right) \\
\left.\left(\alpha_{1,5} \alpha_{3,5} \alpha_{4,5}\right)\left(\alpha_{1,6} \alpha_{3,6} \alpha_{4,6}\right)\right]
\end{gathered}
$$

with $\operatorname{Clos}\left(H_{19,1}\right)=H_{61,1}$ above.

$\mathbf{n}=\mathbf{1 8}, H \cong D_{12}(|H|=12, i=4): \operatorname{rk} N_{H}=16$ and $\left(N_{H}\right)^{*} / N_{H} \cong(\mathbb{Z} / 6 \mathbb{Z})^{4}$.

$$
H_{18,1}=\left[\left(\alpha_{3,1} \alpha_{4,1}\right)\left(\alpha_{3,2} \alpha_{4,2}\right)\left(\alpha_{3,3} \alpha_{4,3}\right)\left(\alpha_{3,4} \alpha_{4,4}\right)\left(\alpha_{1,5} \alpha_{1,6}\right)\left(\alpha_{2,5} \alpha_{2,6}\right)\right.
$$




$$
\begin{gathered}
\left(\alpha_{3,5} \alpha_{4,6}\right)\left(\alpha_{4,5} \alpha_{3,6}\right) \\
\left(\alpha_{1,1} \alpha_{3,2} \alpha_{4,1} \alpha_{1,2} \alpha_{3,1} \alpha_{4,2}\right)\left(\alpha_{2,1} \alpha_{2,2}\right)\left(\alpha_{1,3} \alpha_{3,3} \alpha_{4,3}\right)\left(\alpha_{1,4} \alpha_{3,4} \alpha_{4,4}\right) \\
\left.\left(\alpha_{1,5} \alpha_{3,6} \alpha_{4,5} \alpha_{1,6} \alpha_{3,5} \alpha_{4,6}\right)\left(\alpha_{2,5} \alpha_{2,6}\right)\right]
\end{gathered}
$$

with orbits $\quad\left\{\alpha_{1,1}, \alpha_{3,2}, \alpha_{4,2}, \alpha_{4,1}, \alpha_{3,1}, \alpha_{1,2}\right\}, \quad\left\{\alpha_{2,1}, \alpha_{2,2}\right\}, \quad\left\{\alpha_{1,3}, \alpha_{3,3}, \alpha_{4,3}\right\}$, $\left\{\alpha_{1,4}, \alpha_{3,4}, \alpha_{4,4}\right\},\left\{\alpha_{1,5}, \alpha_{1,6}, \alpha_{3,6}, \alpha_{3,5}, \alpha_{4,5}, \alpha_{4,6}\right\},\left\{\alpha_{2,5}, \alpha_{2,6}\right\}$

$$
\begin{gathered}
H_{18,2}=\left[\left(\alpha_{1,1} \alpha_{4,1}\right)\left(\alpha_{1,2} \alpha_{4,6}\right)\left(\alpha_{2,2} \alpha_{2,6}\right)\left(\alpha_{3,2} \alpha_{3,6}\right)\left(\alpha_{4,2} \alpha_{1,6}\right)\left(\alpha_{1,3} \alpha_{3,3}\right)\right. \\
\left(\alpha_{3,4} \alpha_{4,4}\right)\left(\alpha_{1,5} \alpha_{4,5}\right), \\
\left(\alpha_{1,1} \alpha_{3,2} \alpha_{3,6} \alpha_{4,1} \alpha_{4,2} \alpha_{1,6}\right)\left(\alpha_{2,1} \alpha_{2,2} \alpha_{2,6}\right)\left(\alpha_{3,1} \alpha_{1,2} \alpha_{4,6}\right)\left(\alpha_{1,3} \alpha_{3,3}\right) \\
\left.\left(\alpha_{1,4} \alpha_{1,5} \alpha_{4,4} \alpha_{3,5} \alpha_{3,4} \alpha_{4,5}\right)\left(\alpha_{2,4} \alpha_{2,5}\right)\right]
\end{gathered}
$$

with orbits $\quad\left\{\alpha_{1,1}, \alpha_{4,1}, \alpha_{3,2}, \alpha_{4,2}, \alpha_{3,6}, \alpha_{1,6}\right\}, \quad\left\{\alpha_{2,1}, \alpha_{2,2}, \alpha_{2,6}\right\}, \quad\left\{\alpha_{3,1}, \alpha_{1,2}, \alpha_{4,6}\right\}$, $\left\{\alpha_{1,3}, \alpha_{3,3}\right\},\left\{\alpha_{1,4}, \alpha_{1,5}, \alpha_{4,5}, \alpha_{4,4}, \alpha_{3,4}, \alpha_{3,5}\right\},\left\{\alpha_{2,4}, \alpha_{2,5}\right\}$.

$\mathbf{n}=\mathbf{1 7}, H \cong \mathfrak{A}_{4}(|H|=12, i=3): \operatorname{rk} N_{H}=16$ and $\left(N_{H}\right)^{*} / N_{H} \cong(\mathbb{Z} / 12 \mathbb{Z})^{2} \times(\mathbb{Z} / 2 \mathbb{Z})^{2}$.

$$
\begin{gathered}
H_{17,1}=\left[\left(\alpha_{1,2} \alpha_{1,5} \alpha_{1,6}\right)\left(\alpha_{2,2} \alpha_{2,5} \alpha_{2,6}\right)\left(\alpha_{3,2} \alpha_{3,5} \alpha_{3,6}\right)\left(\alpha_{4,2} \alpha_{4,5} \alpha_{4,6}\right)\right. \\
\left(\alpha_{9} \alpha_{11} \alpha_{12}\right)\left(\alpha_{13} \alpha_{16} \alpha_{15}\right) \\
\left(\alpha_{1,1} \alpha_{1,2}\right)\left(\alpha_{2,1} \alpha_{2,2}\right)\left(\alpha_{3,1} \alpha_{3,2}\right)\left(\alpha_{4,1} \alpha_{4,2}\right)\left(\alpha_{1,5} \alpha_{1,6}\right)\left(\alpha_{2,5} \alpha_{2,6}\right) \\
\left.\left(\alpha_{3,5} \alpha_{3,6}\right)\left(\alpha_{4,5} \alpha_{4,6}\right)\right]
\end{gathered}
$$

with orbits $\quad\left\{\alpha_{1,1}, \alpha_{1,2}, \alpha_{1,5}, \alpha_{1,6}\right\}, \quad\left\{\alpha_{2,1}, \alpha_{2,2}, \alpha_{2,5}, \alpha_{2,6}\right\}, \quad\left\{\alpha_{3,1}, \alpha_{3,2}, \alpha_{3,5}, \alpha_{3,6}\right\}$, $\left\{\alpha_{4,1}, \alpha_{4,2}, \alpha_{4,5}, \alpha_{4,6}\right\},\left\{\alpha_{1,3}, \alpha_{3,3}, \alpha_{4,3}\right\},\left\{\alpha_{1,4}, \alpha_{4,4}, \alpha_{3,4}\right\}$;

$$
\begin{gathered}
H_{17,2}=\left[\left(\alpha_{1,1} \alpha_{3,1} \alpha_{4,1}\right)\left(\alpha_{1,2} \alpha_{3,5} \alpha_{4,6}\right)\left(\alpha_{2,2} \alpha_{2,5} \alpha_{2,6}\right)\left(\alpha_{3,2} \alpha_{4,5} \alpha_{1,6}\right)\right. \\
\left(\alpha_{4,2} \alpha_{1,5} \alpha_{3,6}\right)\left(\alpha_{1,3} \alpha_{4,3} \alpha_{3,3}\right) \\
\left(\alpha_{1,1} \alpha_{1,2}\right)\left(\alpha_{2,1} \alpha_{2,2}\right)\left(\alpha_{3,1} \alpha_{3,2}\right)\left(\alpha_{4,1} \alpha_{4,2}\right)\left(\alpha_{1,5} \alpha_{1,6}\right)\left(\alpha_{2,5} \alpha_{2,6}\right) \\
\left.\left(\alpha_{3,5} \alpha_{3,6}\right)\left(\alpha_{4,5} \alpha_{4,6}\right)\right]
\end{gathered}
$$

with $\quad$ orbits $\left\{\alpha_{1,1}, \alpha_{3,1}, \alpha_{1,2}, \alpha_{4,1}, \alpha_{3,2}, \alpha_{3,5}, \alpha_{4,2}, \alpha_{4,5}, \alpha_{4,6}, \alpha_{3,6}, \alpha_{1,5}, \alpha_{1,6}\right\}$, $\left\{\alpha_{2,1}, \alpha_{2,2}, \alpha_{2,5}, \alpha_{2,6}\right\},\left\{\alpha_{1,3}, \alpha_{4,3}, \alpha_{3,3}\right\}$.

$\mathbf{n}=\mathbf{1 6}, H \cong D_{10}(|H|=10, i=1): \operatorname{rk} N_{H}=16$ and $\left(N_{H}\right)^{*} / N_{H} \cong(\mathbb{Z} / 5 \mathbb{Z})^{4}$.

$$
H_{16,1}=\left[\left(\alpha_{1,2} \alpha_{1,4}\right)\left(\alpha_{2,2} \alpha_{2,4}\right)\left(\alpha_{3,2} \alpha_{3,4}\right)\left(\alpha_{4,2} \alpha_{4,4}\right)\left(\alpha_{1,5} \alpha_{3,6}\right)\left(\alpha_{2,5} \alpha_{2,6}\right)\right.
$$




$$
\begin{gathered}
\left(\alpha_{3,5} \alpha_{4,6}\right)\left(\alpha_{4,5} \alpha_{1,6}\right), \\
\left(\alpha_{1,1} \alpha_{3,2} \alpha_{3,6} \alpha_{1,5} \alpha_{3,4}\right)\left(\alpha_{2,1} \alpha_{2,2} \alpha_{2,6} \alpha_{2,5} \alpha_{2,4}\right)\left(\alpha_{3,1} \alpha_{4,2} \alpha_{4,6} \alpha_{3,5} \alpha_{4,4}\right) \\
\left.\left(\alpha_{4,1} \alpha_{1,2} \alpha_{1,6} \alpha_{4,5} \alpha_{1,4}\right)\right]
\end{gathered}
$$

with orbits $\left\{\alpha_{1,1}, \alpha_{3,2}, \alpha_{3,4}, \alpha_{3,6}, \alpha_{1,5}\right\},\left\{\alpha_{2,1}, \alpha_{2,2}, \alpha_{2,4}, \alpha_{2,6}, \alpha_{2,5}\right\},\left\{\alpha_{3,1}, \alpha_{4,2}, \alpha_{4,4}, \alpha_{4,6}, \alpha_{3,5}\right\}$, $\left\{\alpha_{4,1}, \alpha_{1,2}, \alpha_{1,4}, \alpha_{1,6}, \alpha_{4,5}\right\}$.

$$
\begin{gathered}
\mathbf{n = 1 5}, H \cong C_{3}^{2}(|H|=9, i=2): \\
H_{15,1}=\left[\left(\alpha_{1,1} \alpha_{3,1} \alpha_{4,1}\right)\left(\alpha_{1,2} \alpha_{3,2} \alpha_{4,2}\right)\left(\alpha_{1,3} \alpha_{3,3} \alpha_{4,3}\right)\left(\alpha_{1,4} \alpha_{3,4} \alpha_{4,4}\right)\right. \\
\left(\alpha_{1,5} \alpha_{3,5} \alpha_{4,5}\right)\left(\alpha_{1,6} \alpha_{3,6} \alpha_{4,6}\right), \\
\left(\alpha_{1,1} \alpha_{1,2} \alpha_{1,3}\right)\left(\alpha_{2,1} \alpha_{2,2} \alpha_{2,3}\right)\left(\alpha_{3,1} \alpha_{3,2} \alpha_{3,3}\right)\left(\alpha_{4,1} \alpha_{4,2} \alpha_{4,3}\right) \\
\left.\left(\alpha_{1,5} \alpha_{4,5} \alpha_{3,5}\right)\left(\alpha_{1,6} \alpha_{3,6} \alpha_{4,6}\right)\right]
\end{gathered}
$$

with $\operatorname{Clos}\left(H_{15,1}\right)=H_{30,1}$ above.

$\mathbf{n}=\mathbf{1 0}, H \cong D_{8}(|H|=8, i=3): \operatorname{rk} N_{H}=15$ and $\left(N_{H}\right)^{*} / N_{H} \cong(\mathbb{Z} / 4 \mathbb{Z})^{5}$.

$$
\begin{gathered}
H_{10,1}=\left[\left(\alpha_{1,1} \alpha_{3,5}\right)\left(\alpha_{2,1} \alpha_{2,5}\right)\left(\alpha_{3,1} \alpha_{1,5}\right)\left(\alpha_{4,1} \alpha_{4,5}\right)\left(\alpha_{1,2} \alpha_{3,2}\right)\left(\alpha_{3,3} \alpha_{4,3}\right)\right. \\
\left(\alpha_{1,4} \alpha_{4,4}\right)\left(\alpha_{1,6} \alpha_{3,6}\right), \\
\left(\alpha_{1,1} \alpha_{1,2}\right)\left(\alpha_{2,1} \alpha_{2,2}\right)\left(\alpha_{3,1} \alpha_{3,2}\right)\left(\alpha_{4,1} \alpha_{4,2}\right)\left(\alpha_{1,5} \alpha_{1,6}\right)\left(\alpha_{2,5} \alpha_{2,6}\right) \\
\left.\left(\alpha_{3,5} \alpha_{3,6}\right)\left(\alpha_{4,5} \alpha_{4,6}\right)\right]
\end{gathered}
$$

with orbits $\left\{\alpha_{1,1}, \alpha_{3,5}, \alpha_{1,2}, \alpha_{3,6}, \alpha_{3,2}, \alpha_{1,6}, \alpha_{3,1}, \alpha_{1,5}\right\}, \quad\left\{\alpha_{2,1}, \alpha_{2,5}, \alpha_{2,2}, \alpha_{2,6}\right\}, \quad\left\{\alpha_{4,1}, \alpha_{4,5}\right.$, $\left.\alpha_{4,2}, \alpha_{4,6}\right\},\left\{\alpha_{3,3}, \alpha_{4,3}\right\},\left\{\alpha_{1,4}, \alpha_{4,4}\right\}$.

$\mathbf{n}=7, H \cong C_{6}(|H|=6, i=2):$

$$
\begin{gathered}
H_{7,1}=\left[\left(\alpha_{1,1} \alpha_{3,2} \alpha_{4,1} \alpha_{1,2} \alpha_{3,1} \alpha_{4,2}\right)\left(\alpha_{2,1} \alpha_{2,2}\right)\left(\alpha_{1,3} \alpha_{3,3} \alpha_{4,3}\right)\left(\alpha_{1,4} \alpha_{3,4} \alpha_{4,4}\right)\right. \\
\left.\left(\alpha_{1,5} \alpha_{3,6} \alpha_{4,5} \alpha_{1,6} \alpha_{3,5} \alpha_{4,6}\right)\left(\alpha_{2,5} \alpha_{2,6}\right)\right]
\end{gathered}
$$

with $C l o s\left(H_{7,1}\right)=H_{18,1}$ above;

$$
\begin{gathered}
H_{7,2}=\left[\left(\alpha_{1,1} \alpha_{3,2} \alpha_{3,6} \alpha_{4,1} \alpha_{4,2} \alpha_{1,6}\right)\left(\alpha_{2,1} \alpha_{2,2} \alpha_{2,6}\right)\left(\alpha_{3,1} \alpha_{1,2} \alpha_{4,6}\right)\left(\alpha_{1,3} \alpha_{3,3}\right)\right. \\
\left.\left(\alpha_{1,4} \alpha_{1,5} \alpha_{4,4} \alpha_{3,5} \alpha_{3,4} \alpha_{4,5}\right)\left(\alpha_{2,4} \alpha_{2,5}\right)\right]
\end{gathered}
$$

with $C l o s\left(H_{7,2}\right)=H_{18,2}$ above. 
$\mathbf{n}=6, H \cong D_{6}(|H|=6, i=1): \operatorname{rk} N_{H}=14$ and $\left(N_{H}\right)^{*} / N_{H} \cong(\mathbb{Z} / 6 \mathbb{Z})^{2} \times(\mathbb{Z} / 3 \mathbb{Z})^{3}$.

$$
\begin{gathered}
H_{6,1}=\left[\left(\alpha_{1,1} \alpha_{4,2}\right)\left(\alpha_{2,1} \alpha_{2,2}\right)\left(\alpha_{3,1} \alpha_{3,2}\right)\left(\alpha_{4,1} \alpha_{1,2}\right)\left(\alpha_{1,3} \alpha_{4,3}\right)\left(\alpha_{1,4} \alpha_{4,4}\right)\right. \\
\left(\alpha_{1,5} \alpha_{4,5}\right)\left(\alpha_{1,6} \alpha_{4,6}\right) \\
\left(\alpha_{1,1} \alpha_{3,2}\right)\left(\alpha_{2,1} \alpha_{2,2}\right)\left(\alpha_{3,1} \alpha_{1,2}\right)\left(\alpha_{4,1} \alpha_{4,2}\right)\left(\alpha_{9} \alpha_{11}\right)\left(\alpha_{13} \alpha_{15}\right) \\
\left.\left(\alpha_{1,5} \alpha_{3,5}\right)\left(\alpha_{1,6} \alpha_{3,6}\right)\right]
\end{gathered}
$$

with orbits $\left\{\alpha_{1,1}, \alpha_{4,2}, \alpha_{3,2}, \alpha_{4,1}, \alpha_{3,1}, \alpha_{1,2}\right\}, \quad\left\{\alpha_{2,1}, \alpha_{2,2}\right\}, \quad\left\{\alpha_{1,3}, \alpha_{4,3}, \alpha_{3,3}\right\}, \quad\left\{\alpha_{1,4}, \alpha_{4,4}, \alpha_{3,4}\right\}$, $\left\{\alpha_{1,5}, \alpha_{4,5}, \alpha_{3,5}\right\},\left\{\alpha_{1,6}, \alpha_{4,6}, \alpha_{3,6}\right\}$

$$
\begin{gathered}
H_{6,2}=\left[\left(\alpha_{1,1} \alpha_{3,3}\right)\left(\alpha_{2,1} \alpha_{2,3}\right)\left(\alpha_{3,1} \alpha_{1,3}\right)\left(\alpha_{4,1} \alpha_{4,3}\right)\left(\alpha_{1,2} \alpha_{3,2}\right)\left(\alpha_{1,4} \alpha_{3,4}\right)\right. \\
\left(\alpha_{3,5} \alpha_{4,5}\right)\left(\alpha_{1,6} \alpha_{4,6}\right) \\
\left(\alpha_{1,1}, \alpha_{3,2}\right)\left(\alpha_{2,1}, \alpha_{2,2}\right)\left(\alpha_{3,1}, \alpha_{1,2}\right)\left(\alpha_{4,1}, \alpha_{4,2}\right)\left(\alpha_{1,3} \alpha_{3,3}\right)\left(\alpha_{1,4} \alpha_{3,4}\right) \\
\left.\left.\left(\alpha_{1,5} \alpha_{3,5}\right)\left(\alpha_{1,6} \alpha_{3,6}\right)\right]\right)
\end{gathered}
$$

with orbits $\quad\left\{\alpha_{1,1}, \alpha_{3,3}, \alpha_{3,2}, \alpha_{1,3}, \alpha_{1,2}, \alpha_{3,1}\right\}, \quad\left\{\alpha_{2,1}, \alpha_{2,3}, \alpha_{2,2}\right\}, \quad\left\{\alpha_{4,1}, \alpha_{4,3}, \alpha_{4,2}\right\}$, $\left\{\alpha_{1,4}, \alpha_{3,4}\right\},\left\{\alpha_{1,5}, \alpha_{3,5}, \alpha_{4,5}\right\},\left\{\alpha_{1,6}, \alpha_{4,6}, \alpha_{3,6}\right\}$

$$
\begin{gathered}
H_{6,3}=\left[\left(\alpha_{1,1} \alpha_{3,2}\right)\left(\alpha_{2,1} \alpha_{2,2}\right)\left(\alpha_{3,1} \alpha_{4,2}\right)\left(\alpha_{4,1} \alpha_{1,2}\right)\left(\alpha_{1,4} \alpha_{1,6}\right)\left(\alpha_{2,4} \alpha_{2,6}\right)\right. \\
\left(\alpha_{3,4} \alpha_{3,6}\right)\left(\alpha_{4,4} \alpha_{4,6}\right) \\
\left(\alpha_{1,1} \alpha_{1,2}\right)\left(\alpha_{2,1} \alpha_{2,2}\right)\left(\alpha_{3,1} \alpha_{3,2}\right)\left(\alpha_{4,1} \alpha_{4,2}\right)\left(\alpha_{1,5} \alpha_{1,6}\right)\left(\alpha_{2,5} \alpha_{2,6}\right) \\
\left.\left(\alpha_{3,5} \alpha_{3,6}\right)\left(\alpha_{4,5} \alpha_{4,6}\right)\right]
\end{gathered}
$$

with orbits $\left\{\alpha_{1,1}, \alpha_{3,2}, \alpha_{1,2}, \alpha_{3,1}, \alpha_{4,1}, \alpha_{4,2}\right\},\left\{\alpha_{2,1}, \alpha_{2,2}\right\}, \quad\left\{\alpha_{1,4}, \alpha_{1,6}, \alpha_{1,5}\right\}, \quad\left\{\alpha_{2,4}, \alpha_{2,6}, \alpha_{2,5}\right\}$, $\left\{\alpha_{3,4}, \alpha_{3,6}, \alpha_{3,5}\right\},\left\{\alpha_{4,4}, \alpha_{4,6}, \alpha_{4,5}\right\}$.

$$
\begin{gathered}
\mathbf{n}=\mathbf{5}, H \cong C_{5}(|H|=5, i=1): \\
H_{5,1}=\left[\left(\alpha_{1,1} \alpha_{3,2} \alpha_{3,6} \alpha_{1,5} \alpha_{3,4}\right)\left(\alpha_{2,1} \alpha_{2,2} \alpha_{2,6} \alpha_{2,5} \alpha_{2,4}\right)\left(\alpha_{3,1} \alpha_{4,2} \alpha_{4,6} \alpha_{3,5} \alpha_{4,4}\right)\right. \\
\left.\left(\alpha_{4,1} \alpha_{1,2} \alpha_{1,6} \alpha_{4,5} \alpha_{1,4}\right)\right]
\end{gathered}
$$

with $\operatorname{Clos}\left(H_{5,1}\right)=H_{16,1}$ above.

$\mathbf{n}=4, H \cong C_{4}(|H|=4, i=1): \operatorname{rk} N_{H}=14$ and $\left(N_{H}\right)^{*} / N_{H} \cong(\mathbb{Z} / 4 \mathbb{Z})^{4} \times(\mathbb{Z} / 2 \mathbb{Z})^{2}$.

$$
H_{4,1}=\left[\left(\alpha_{1,1} \alpha_{3,2} \alpha_{1,6} \alpha_{3,5}\right)\left(\alpha_{2,1} \alpha_{2,2} \alpha_{2,6} \alpha_{2,5}\right)\left(\alpha_{3,1} \alpha_{1,2} \alpha_{3,6} \alpha_{1,5}\right)\right.
$$




$$
\left.\left(\alpha_{4,1} \alpha_{4,2} \alpha_{4,6} \alpha_{4,5}\right)\left(\alpha_{1,3} \alpha_{4,3}\right)\left(\alpha_{3,4} \alpha_{4,4}\right)\right]
$$

with orbits $\quad\left\{\alpha_{1,1}, \alpha_{3,2}, \alpha_{1,6}, \alpha_{3,5}\right\}, \quad\left\{\alpha_{2,1}, \alpha_{2,2}, \alpha_{2,6}, \alpha_{2,5}\right\}, \quad\left\{\alpha_{3,1}, \alpha_{1,2}, \alpha_{3,6}, \alpha_{1,5}\right\}$, $\left\{\alpha_{4,1}, \alpha_{4,2}, \alpha_{4,6}, \alpha_{4,5}\right\},\left\{\alpha_{1,3}, \alpha_{4,3}\right\},\left\{\alpha_{3,4}, \alpha_{4,4}\right\}$.

$\mathbf{n}=\mathbf{3}, H \cong C_{2}^{2}(|H|=4, i=2): \operatorname{rk} N_{H}=12$ and $\left(N_{H}\right)^{*} / N_{H} \cong(\mathbb{Z} / 4 \mathbb{Z})^{2} \times(\mathbb{Z} / 2 \mathbb{Z})^{6}$.

$$
\begin{gathered}
H_{3,1}=\left[\left(\alpha_{1,1} \alpha_{3,1}\right)\left(\alpha_{1,2} \alpha_{3,2}\right)\left(\alpha_{1,3} \alpha_{3,4}\right)\left(\alpha_{2,3} \alpha_{2,4}\right)\left(\alpha_{3,3} \alpha_{1,4}\right)\left(\alpha_{4,3} \alpha_{4,4}\right)\right. \\
\left(\alpha_{1,5} \alpha_{3,5}\right)\left(\alpha_{1,6} \alpha_{3,6}\right), \\
\left(\alpha_{1,1} \alpha_{3,2}\right)\left(\alpha_{2,1} \alpha_{2,2}\right)\left(\alpha_{3,1} \alpha_{1,2}\right)\left(\alpha_{4,1} \alpha_{4,2}\right)\left(\alpha_{1,3} \alpha_{3,3}\right)\left(\alpha_{1,4} \alpha_{3,4}\right) \\
\left.\left(\alpha_{1,5} \alpha_{3,5}\right)\left(\alpha_{1,6} \alpha_{3,6}\right)\right]
\end{gathered}
$$

with orbits $\quad\left\{\alpha_{1,1}, \alpha_{3,1}, \alpha_{3,2}, \alpha_{1,2}\right\}, \quad\left\{\alpha_{2,1}, \alpha_{2,2}\right\}, \quad\left\{\alpha_{4,1}, \alpha_{4,2}\right\}, \quad\left\{\alpha_{1,3}, \alpha_{3,4}, \alpha_{3,3}, \alpha_{1,4}\right\}$, $\left\{\alpha_{2,3}, \alpha_{2,4}\right\},\left\{\alpha_{4,3}, \alpha_{4,4}\right\},\left\{\alpha_{1,5}, \alpha_{3,5}\right\},\left\{\alpha_{1,6}, \alpha_{3,6}\right\}$

$$
\begin{gathered}
H_{3,2}=\left[\left(\alpha_{1,1} \alpha_{1,5}\right)\left(\alpha_{2,1} \alpha_{2,5}\right)\left(\alpha_{3,1} \alpha_{3,5}\right)\left(\alpha_{4,1} \alpha_{4,5}\right)\left(\alpha_{1,2} \alpha_{1,6}\right)\left(\alpha_{2,2} \alpha_{2,6}\right)\right. \\
\left(\alpha_{3,2} \alpha_{3,6}\right)\left(\alpha_{4,2} \alpha_{4,6}\right), \\
\left(\alpha_{1,1} \alpha_{1,2}\right)\left(\alpha_{2,1} \alpha_{2,2}\right)\left(\alpha_{3,1} \alpha_{3,2}\right)\left(\alpha_{4,1} \alpha_{4,2}\right)\left(\alpha_{1,5} \alpha_{1,6}\right)\left(\alpha_{2,5} \alpha_{2,6}\right) \\
\left.\left(\alpha_{3,5} \alpha_{3,6}\right)\left(\alpha_{4,5} \alpha_{4,6}\right)\right]
\end{gathered}
$$

with orbits $\quad\left\{\alpha_{1,1}, \alpha_{1,5}, \alpha_{1,2}, \alpha_{1,6}\right\}, \quad\left\{\alpha_{2,1}, \alpha_{2,5}, \alpha_{2,2}, \alpha_{2,6}\right\}, \quad\left\{\alpha_{3,1}, \alpha_{3,5}, \alpha_{3,2}, \alpha_{3,6}\right\}$, $\left\{\alpha_{4,1}, \alpha_{4,5}, \alpha_{4,2}, \alpha_{4,6}\right\}$

$$
\begin{gathered}
H_{3,3}=\left[\left(\alpha_{1,1} \alpha_{1,2}\right)\left(\alpha_{2,1} \alpha_{2,2}\right)\left(\alpha_{3,1} \alpha_{3,2}\right)\left(\alpha_{4,1} \alpha_{4,2}\right)\left(\alpha_{1,3} \alpha_{1,4}\right)\left(\alpha_{2,3} \alpha_{2,4}\right)\right. \\
\left(\alpha_{3,3} \alpha_{3,4}\right)\left(\alpha_{4,3} \alpha_{4,4}\right), \\
\left(\alpha_{1,1} \alpha_{1,2}\right)\left(\alpha_{2,1} \alpha_{2,2}\right)\left(\alpha_{3,1} \alpha_{3,2}\right)\left(\alpha_{4,1} \alpha_{4,2}\right)\left(\alpha_{1,5} \alpha_{1,6}\right)\left(\alpha_{2,5} \alpha_{2,6}\right) \\
\left.\left(\alpha_{3,5} \alpha_{3,6}\right)\left(\alpha_{4,5} \alpha_{4,6}\right)\right]
\end{gathered}
$$

with orbits $\left\{\alpha_{1,1}, \alpha_{1,2}\right\}, \quad\left\{\alpha_{2,1}, \alpha_{2,2}\right\}, \quad\left\{\alpha_{3,1}, \alpha_{3,2}\right\}, \quad\left\{\alpha_{4,1}, \alpha_{4,2}\right\}, \quad\left\{\alpha_{1,3}, \alpha_{1,4}\right\}, \quad\left\{\alpha_{2,3}, \alpha_{2,4}\right\}$, $\left\{\alpha_{3,3}, \alpha_{3,4}\right\},\left\{\alpha_{4,3}, \alpha_{4,4}\right\},\left\{\alpha_{1,5}, \alpha_{1,6}\right\},\left\{\alpha_{2,5}, \alpha_{2,6}\right\},\left\{\alpha_{3,5}, \alpha_{3,6}\right\},\left\{\alpha_{4,5}, \alpha_{4,6}\right\}$.

$\mathbf{n}=\mathbf{2}, H \cong C_{3}(|H|=3, i=1): \operatorname{rk} N_{H}=12$ and $\left(N_{H}\right)^{*} / N_{H} \cong(\mathbb{Z} / 3 \mathbb{Z})^{6}$.

$$
\begin{gathered}
H_{2,1}=\left[\left(\alpha_{1,1} \alpha_{3,1} \alpha_{4,1}\right)\left(\alpha_{1,2} \alpha_{3,2} \alpha_{4,2}\right)\left(\alpha_{1,3} \alpha_{3,3} \alpha_{4,3}\right)\left(\alpha_{1,4} \alpha_{3,4} \alpha_{4,4}\right)\right. \\
\left.\left(\alpha_{1,5} \alpha_{3,5} \alpha_{4,5}\right)\left(\alpha_{1,6} \alpha_{3,6} \alpha_{4,6}\right)\right]
\end{gathered}
$$


with orbits $\quad\left\{\alpha_{1,1}, \alpha_{3,1}, \alpha_{4,1}\right\}, \quad\left\{\alpha_{1,2}, \alpha_{3,2}, \alpha_{4,2}\right\}, \quad\left\{\alpha_{1,3}, \alpha_{3,3}, \alpha_{4,3}\right\}, \quad\left\{\alpha_{1,4}, \alpha_{3,4}, \alpha_{4,4}\right\}$, $\left\{\alpha_{1,5}, \alpha_{3,5}, \alpha_{4,5}\right\},\left\{\alpha_{1,6}, \alpha_{3,6}, \alpha_{4,6}\right\}$

$$
\begin{gathered}
H_{2,2}=\left[\left(\alpha_{1,1} \alpha_{3,2} \alpha_{4,6}\right)\left(\alpha_{2,1} \alpha_{2,2} \alpha_{2,6}\right)\left(\alpha_{3,1} \alpha_{4,2} \alpha_{1,6}\right)\left(\alpha_{4,1} \alpha_{1,2} \alpha_{3,6}\right)\right. \\
\left.\left(\alpha_{1,3} \alpha_{4,3} \alpha_{3,3}\right)\left(\alpha_{1,5} \alpha_{3,5} \alpha_{4,5}\right)\right]
\end{gathered}
$$

with orbits $\quad\left\{\alpha_{1,1}, \alpha_{3,2}, \alpha_{4,6}\right\}, \quad\left\{\alpha_{2,1}, \alpha_{2,2}, \alpha_{2,6}\right\}, \quad\left\{\alpha_{3,1}, \alpha_{4,2}, \alpha_{1,6}\right\}, \quad\left\{\alpha_{4,1}, \alpha_{1,2}, \alpha_{3,6}\right\}$, $\left\{\alpha_{1,3}, \alpha_{4,3}, \alpha_{3,3}\right\},\left\{\alpha_{1,5}, \alpha_{3,5}, \alpha_{4,5}\right\}$.

$$
\mathbf{n}=\mathbf{1}, H \cong C_{2}(|H|=2, i=1): \operatorname{rk} N_{H}=8 \text { and }\left(N_{H}\right)^{*} / N_{H} \cong(\mathbb{Z} / 2 \mathbb{Z})^{8} \text {. }
$$

$$
\begin{gathered}
H_{1,1}=\left[\left(\alpha_{1,1} \alpha_{3,2}\right)\left(\alpha_{2,1} \alpha_{2,2}\right)\left(\alpha_{3,1} \alpha_{1,2}\right)\left(\alpha_{4,1} \alpha_{4,2}\right)\left(\alpha_{1,3} \alpha_{3,3}\right)\left(\alpha_{1,4} \alpha_{3,4}\right)\right. \\
\left.\left(\alpha_{1,5} \alpha_{3,5}\right)\left(\alpha_{1,6} \alpha_{3,6}\right)\right]
\end{gathered}
$$

with orbits $\left\{\alpha_{1,1}, \alpha_{3,2}\right\}, \quad\left\{\alpha_{2,1}, \alpha_{2,2}\right\}, \quad\left\{\alpha_{3,1}, \alpha_{1,2}\right\}, \quad\left\{\alpha_{4,1}, \alpha_{4,2}\right\}, \quad\left\{\alpha_{1,3}, \alpha_{3,3}\right\}, \quad\left\{\alpha_{1,4}, \alpha_{3,4}\right\}$, $\left\{\alpha_{1,5}, \alpha_{3,5}\right\},\left\{\alpha_{1,6}, \alpha_{3,6}\right\}$;

$$
\begin{gathered}
H_{1,2}=\left[\left(\alpha_{1,1} \alpha_{1,2}\right)\left(\alpha_{2,1} \alpha_{2,2}\right)\left(\alpha_{3,1} \alpha_{3,2}\right)\left(\alpha_{4,1} \alpha_{4,2}\right)\left(\alpha_{1,5} \alpha_{1,6}\right)\left(\alpha_{2,5} \alpha_{2,6}\right)\right. \\
\left.\left(\alpha_{3,5} \alpha_{3,6}\right)\left(\alpha_{4,5} \alpha_{4,6}\right)\right]
\end{gathered}
$$

with orbits $\left\{\alpha_{1,1}, \alpha_{1,2}\right\}, \quad\left\{\alpha_{2,1}, \alpha_{2,2}\right\}, \quad\left\{\alpha_{3,1}, \alpha_{3,2}\right\}, \quad\left\{\alpha_{4,1}, \alpha_{4,2}\right\}, \quad\left\{\alpha_{1,5}, \alpha_{1,6}\right\}, \quad\left\{\alpha_{2,5}, \alpha_{2,6}\right\}$, $\left\{\alpha_{3,5}, \alpha_{3,6}\right\},\left\{\alpha_{4,5}, \alpha_{4,6}\right\}$;

In [17] and [18], these conjugacy classes were described by direct considerations. Here is the correspondence between these two descriptions: $H_{70,1} \cong\left(\right.$ II.1); $H_{61,1} \cong$ (I.1); $H_{55,1} \cong$ (II.2); $H_{48,1} \cong\left(\mathrm{I}^{\prime} .1\right) ; H_{47,1} \cong(\mathrm{I} .2) ; H_{36,1} \cong(\mathrm{I} .3) ; H_{34,1} \cong\left(\mathrm{II} .6^{\prime}\right) ; H_{34,2} \cong(\mathrm{II} .6) ; H_{32,1} \cong$

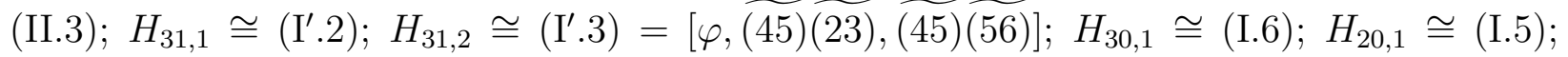
$H_{19,1} \cong($ I. 4$) ; H_{18,1} \cong\left(\right.$ I.8); $H_{18,2} \cong\left(\right.$ II.8); $H_{17,1} \cong\left(\right.$ II.7'); $H_{17,2} \cong$ (II.7); $H_{16,1} \cong$ (II.4); $H_{15,1} \cong$ (I.7); $H_{10,1} \cong\left(\right.$ II.12); $H_{7,1} \cong\left(\right.$ I.9); $H_{7,2} \cong\left(\right.$ II.9); $H_{6,1} \cong\left(\right.$ I.10); $H_{6,2} \cong($ II.11); $H_{6,3} \cong\left(\right.$ II.10); $H_{5,1} \cong($ II. $) ; H_{4,1} \cong\left(\right.$ II.13); $H_{3,1} \cong\left(\right.$ II.16); $H_{3,2} \cong\left(\right.$ II.15); $H_{3,3} \cong($ II'.1); $H_{2,1} \cong(\mathrm{I} .11) ; H_{2,2} \cong(\mathrm{II} .14) ; H_{1,1} \cong(\mathrm{II} .18) ; H_{1,2} \cong(\mathrm{II} .17)$.

Case 18. For the Niemeier lattice

$$
\begin{gathered}
N=N_{18}=N\left(4 A_{5} \oplus D_{4}\right)=\left[4 A_{5} \oplus D_{4}, 2 \varepsilon_{1,1}+2 \varepsilon_{1,3}+4 \varepsilon_{1,4}, 2 \varepsilon_{1,1}+4 \varepsilon_{1,2}+2 \varepsilon_{1,4},\right. \\
\left.2 \varepsilon_{1,1}+2 \varepsilon_{1,2}+4 \varepsilon_{1,3}, 3 \varepsilon_{1,1}+3 \varepsilon_{1,2}+\varepsilon_{1,5}, 3 \varepsilon_{1,1}+3 \varepsilon_{1,3}+\varepsilon_{2,5}, 3 \varepsilon_{1,1}+3 \varepsilon_{1,4}+\varepsilon_{3,5}\right]
\end{gathered}
$$


the group $A=A(N)$ has the order 48 , and it is generated by involutions

$$
\begin{gathered}
\varphi_{0}=\left(\alpha_{1,1} \alpha_{5,1}\right)\left(\alpha_{1,2} \alpha_{5,2}\right)\left(\alpha_{1,3} \alpha_{5,3}\right)\left(\alpha_{1,4} \alpha_{5,4}\right)\left(\alpha_{2,1} \alpha_{4,1}\right)\left(\alpha_{2,2} \alpha_{4,2}\right) \\
\left(\alpha_{2,3} \alpha_{4,3}\right)\left(\alpha_{2,4} \alpha_{4,4}\right), \\
\widetilde{(12)}=\left(\alpha_{1,1} \alpha_{1,2}\right)\left(\alpha_{5,1} \alpha_{5,2}\right)\left(\alpha_{1,4} \alpha_{5,4}\right)\left(\alpha_{1,5} \alpha_{3,5}\right)\left(\alpha_{2,1} \alpha_{2,2}\right)\left(\alpha_{4,1} \alpha_{4,2}\right) \\
\left(\alpha_{2,4} \alpha_{4,4}\right)\left(\alpha_{3,1} \alpha_{3,2}\right), \\
\widetilde{(23)}=\left(\alpha_{1,1} \alpha_{5,1}\right)\left(\alpha_{1,2} \alpha_{1,3}\right)\left(\alpha_{5,2} \alpha_{5,3}\right)\left(\alpha_{1,5} \alpha_{4,5}\right)\left(\alpha_{2,1} \alpha_{4,1}\right)\left(\alpha_{2,2} \alpha_{2,3}\right) \\
\left(\alpha_{4,2} \alpha_{4,3}\right)\left(\alpha_{3,2} \alpha_{3,3}\right), \\
\widetilde{(34)=}\left(\alpha_{1,1} \alpha_{5,1}\right)\left(\alpha_{1,3} \alpha_{1,4}\right)\left(\alpha_{5,3} \alpha_{5,4}\right)\left(\alpha_{1,5} \alpha_{3,5}\right)\left(\alpha_{2,1} \alpha_{4,1}\right)\left(\alpha_{2,3} \alpha_{2,4}\right) \\
\left(\alpha_{4,3} \alpha_{4,4}\right)\left(\alpha_{3,3} \alpha_{3,4}\right) .
\end{gathered}
$$

(see [5, Ch. 16] and [17, [18]).

\section{Classification of KahK3 conjugacy classes for $A\left(N_{18}\right)$ :}

$$
\begin{gathered}
\mathbf{n}=\mathbf{5 4 , H \cong} T_{48}(|H|=48, i=29): \operatorname{rk} N_{H}=19 \text { and }\left(N_{H}\right)^{*} / N_{H} \cong \mathbb{Z} / 24 \mathbb{Z} \times \mathbb{Z} / 8 \mathbb{Z} \times \mathbb{Z} / 2 \mathbb{Z} . \\
H_{54,1}=\left[\left(\alpha_{1,2} \alpha_{1,4} \alpha_{1,3}\right)\left(\alpha_{2,2} \alpha_{2,4} \alpha_{2,3}\right)\left(\alpha_{3,2} \alpha_{3,4} \alpha_{3,3}\right)\left(\alpha_{4,2} \alpha_{4,4} \alpha_{4,3}\right)\right. \\
\left(\alpha_{5,2} \alpha_{5,4} \alpha_{5,3}\right)\left(\alpha_{1,5} \alpha_{4,5} \alpha_{3,5}\right), \\
\left(\alpha_{1,1} \alpha_{1,2} \alpha_{1,3} \alpha_{5,4} \alpha_{5,1} \alpha_{5,2} \alpha_{5,3} \alpha_{1,4}\right)\left(\alpha_{2,1} \alpha_{2,2} \alpha_{2,3} \alpha_{4,4} \alpha_{4,1} \alpha_{4,2} \alpha_{4,3} \alpha_{2,4}\right) \\
\left.\left(\alpha_{3,1} \alpha_{3,2} \alpha_{3,3} \alpha_{3,4}\right)\left(\alpha_{3,5} \alpha_{4,5}\right)\right]
\end{gathered}
$$

with $\quad$ orbits $\quad\left\{\alpha_{1,1}, \alpha_{1,2}, \alpha_{1,4}, \alpha_{1,3}, \alpha_{5,4}, \alpha_{5,3}, \alpha_{5,1}, \alpha_{5,2}\right\}, \quad\left\{\alpha_{2,1}, \alpha_{2,2}, \alpha_{2,4}, \alpha_{2,3}, \alpha_{4,4}, \alpha_{4,3}\right.$, $\left.\alpha_{4,1}, \alpha_{4,2}\right\},\left\{\alpha_{3,1}, \alpha_{3,2}, \alpha_{3,4}, \alpha_{3,3}\right\},\left\{\alpha_{1,5}, \alpha_{4,5}, \alpha_{3,5}\right\}$.

$$
\begin{gathered}
\mathbf{n}=\mathbf{3 8}, H \cong T_{24}(|H|=24, i=3): \\
H_{38,1}=\left[\left(\alpha_{1,2} \alpha_{1,4} \alpha_{1,3}\right)\left(\alpha_{2,2} \alpha_{2,4} \alpha_{2,3}\right)\left(\alpha_{3,2} \alpha_{3,4} \alpha_{3,3}\right)\left(\alpha_{4,2} \alpha_{4,4} \alpha_{4,3}\right)\right. \\
\left(\alpha_{5,2} \alpha_{5,4} \alpha_{5,3}\right)\left(\alpha_{1,5} \alpha_{4,5} \alpha_{3,5}\right), \\
\left(\alpha_{1,1} \alpha_{1,2} \alpha_{5,1} \alpha_{5,2}\right)\left(\alpha_{2,1} \alpha_{2,2} \alpha_{4,1} \alpha_{4,2}\right)\left(\alpha_{3,1} \alpha_{3,2}\right)\left(\alpha_{1,3} \alpha_{1,4} \alpha_{5,3} \alpha_{5,4}\right) \\
\left.\left(\alpha_{2,3} \alpha_{2,4} \alpha_{4,3} \alpha_{4,4}\right)\left(\alpha_{3,3} \alpha_{3,4}\right)\right]
\end{gathered}
$$

with $\operatorname{Clos}\left(H_{38,1}\right)=H_{54,1}$ above. 
$\mathbf{n}=\mathbf{2 6}, H \cong S D_{16}(|H|=16, i=8): \operatorname{rk} N_{H}=18$ and $\left(N_{H}\right)^{*} / N_{H} \cong(\mathbb{Z} / 8 \mathbb{Z})^{2} \times \mathbb{Z} / 4 \mathbb{Z} \times$ $\mathbb{Z} / 2 \mathbb{Z}$.

$$
\begin{gathered}
H_{26,1}=\left[\left(\alpha_{1,1} \alpha_{1,3} \alpha_{5,1} \alpha_{5,3}\right)\left(\alpha_{2,1} \alpha_{2,3} \alpha_{4,1} \alpha_{4,3}\right)\left(\alpha_{3,1} \alpha_{3,3}\right)\left(\alpha_{1,2} \alpha_{5,4} \alpha_{5,2} \alpha_{1,4}\right)\right. \\
\left(\alpha_{2,2} \alpha_{4,4} \alpha_{4,2} \alpha_{2,4}\right)\left(\alpha_{3,2} \alpha_{3,4}\right) \\
\left(\alpha_{1,2} \alpha_{5,2}\right)\left(\alpha_{2,2} \alpha_{4,2}\right)\left(\alpha_{1,3} \alpha_{5,4}\right)\left(\alpha_{2,3} \alpha_{4,4}\right)\left(\alpha_{3,3} \alpha_{3,4}\right)\left(\alpha_{4,3} \alpha_{2,4}\right) \\
\left.\left(\alpha_{5,3} \alpha_{1,4}\right)\left(\alpha_{1,5} \alpha_{3,5}\right)\right]
\end{gathered}
$$

with orbits $\quad\left\{\alpha_{1,1}, \alpha_{1,3}, \alpha_{5,1}, \alpha_{5,4}, \alpha_{5,3}, \alpha_{5,2}, \alpha_{1,4}, \alpha_{1,2}\right\}, \quad\left\{\alpha_{2,1}, \alpha_{2,3}, \alpha_{4,1}, \alpha_{4,4}, \alpha_{4,3}, \alpha_{4,2}\right.$, $\left.\alpha_{2,4}, \alpha_{2,2}\right\},\left\{\alpha_{3,1}, \alpha_{3,3}, \alpha_{3,4}, \alpha_{3,2}\right\},\left\{\alpha_{1,5}, \alpha_{3,5}\right\}$.

$\mathbf{n}=\mathbf{1 8}, H \cong D_{12}(|H|=12, i=4): \operatorname{rk} N_{H}=16$ and $\left(N_{H}\right)^{*} / N_{H} \cong(\mathbb{Z} / 6 \mathbb{Z})^{4}$.

$$
\begin{gathered}
H_{18,1}=\left[\left(\alpha_{1,2} \alpha_{5,2}\right)\left(\alpha_{2,2} \alpha_{4,2}\right)\left(\alpha_{1,3} \alpha_{5,4}\right)\left(\alpha_{2,3} \alpha_{4,4}\right)\left(\alpha_{3,3} \alpha_{3,4}\right)\left(\alpha_{4,3} \alpha_{2,4}\right)\right. \\
\left(\alpha_{5,3} \alpha_{1,4}\right)\left(\alpha_{1,5} \alpha_{3,5}\right) \\
\left(\alpha_{1,1} \alpha_{5,1}\right)\left(\alpha_{2,1} \alpha_{4,1}\right)\left(\alpha_{1,2} \alpha_{5,3} \alpha_{1,4} \alpha_{5,2} \alpha_{1,3} \alpha_{5,4}\right)\left(\alpha_{2,2} \alpha_{4,3} \alpha_{2,4} \alpha_{4,2} \alpha_{2,3} \alpha_{4,4}\right) \\
\left.\left(\alpha_{3,2} \alpha_{3,3} \alpha_{3,4}\right)\left(\alpha_{1,5} \alpha_{3,5} \alpha_{4,5}\right)\right]
\end{gathered}
$$

with orbits $\left\{\alpha_{1,1}, \alpha_{5,1}\right\}, \quad\left\{\alpha_{2,1}, \alpha_{4,1}\right\}, \quad\left\{\alpha_{1,2}, \alpha_{5,2}, \alpha_{5,3}, \alpha_{1,3}, \alpha_{1,4}, \alpha_{5,4}\right\}, \quad\left\{\alpha_{2,2}, \alpha_{4,2}, \alpha_{4,3}\right.$, $\left.\alpha_{2,3}, \alpha_{2,4}, \alpha_{4,4}\right\},\left\{\alpha_{3,2}, \alpha_{3,3}, \alpha_{3,4}\right\},\left\{\alpha_{1,5}, \alpha_{3,5}, \alpha_{4,5}\right\}$.

$\mathbf{n}=\mathbf{1 4}, H \cong C_{8}(|H|=8, i=1)$ :

$$
\begin{gathered}
H_{14,1}=\left[\left(\alpha_{1,1} \alpha_{1,3} \alpha_{5,2} \alpha_{5,4} \alpha_{5,1} \alpha_{5,3} \alpha_{1,2} \alpha_{1,4}\right)\left(\alpha_{2,1} \alpha_{2,3} \alpha_{4,2} \alpha_{4,4} \alpha_{4,1} \alpha_{4,3} \alpha_{2,2} \alpha_{2,4}\right)\right. \\
\left.\left(\alpha_{3,1} \alpha_{3,3} \alpha_{3,2} \alpha_{3,4}\right)\left(\alpha_{1,5} \alpha_{3,5}\right)\right]
\end{gathered}
$$

with $\operatorname{Clos}\left(H_{14,1}\right)=H_{26,1}$ above.

$\mathbf{n}=\mathbf{1 2}, H \cong Q_{8}(|H|=8, i=4): \operatorname{rk} N_{H}=17,\left(N_{H}\right)^{*} / N_{H} \cong(\mathbb{Z} / 8 \mathbb{Z})^{2} \times(\mathbb{Z} / 2 \mathbb{Z})^{3}$.

$$
\begin{gathered}
H_{12,1}=\left[\left(\alpha_{1,1} \alpha_{5,4} \alpha_{5,1} \alpha_{1,4}\right)\left(\alpha_{2,1} \alpha_{4,4} \alpha_{4,1} \alpha_{2,4}\right)\left(\alpha_{3,1} \alpha_{3,4}\right)\left(\alpha_{1,2} \alpha_{5,3} \alpha_{5,2} \alpha_{1,3}\right)\right. \\
\left(\alpha_{2,2} \alpha_{4,3} \alpha_{4,2} \alpha_{2,3}\right)\left(\alpha_{3,2} \alpha_{3,3}\right) \\
\left(\alpha_{1,1} \alpha_{1,2} \alpha_{5,1} \alpha_{5,2}\right)\left(\alpha_{2,1} \alpha_{2,2} \alpha_{4,1} \alpha_{4,2}\right)\left(\alpha_{3,1} \alpha_{3,2}\right)\left(\alpha_{1,3} \alpha_{1,4} \alpha_{5,3} \alpha_{5,4}\right) \\
\left.\left(\alpha_{2,3} \alpha_{2,4} \alpha_{4,3} \alpha_{4,4}\right)\left(\alpha_{3,3} \alpha_{3,4}\right)\right]
\end{gathered}
$$

with orbits $\left\{\alpha_{1,1}, \alpha_{5,4}, \alpha_{1,2}, \alpha_{5,1}, \alpha_{1,3}, \alpha_{5,3}, \alpha_{1,4}, \alpha_{5,2}\right\},\left\{\alpha_{2,1}, \alpha_{4,4}, \alpha_{2,2}, \alpha_{4,1}, \alpha_{2,3}, \alpha_{4,3}, \alpha_{2,4}, \alpha_{4,2}\right\}$, $\left\{\alpha_{3,1}, \alpha_{3,4}, \alpha_{3,2}, \alpha_{3,3}\right\}$. 
$\mathbf{n}=\mathbf{1 0}, H \cong D_{8}(|H|=8, i=3): \operatorname{rk} N_{H}=15$ and $\left(N_{H}\right)^{*} / N_{H} \cong(\mathbb{Z} / 4 \mathbb{Z})^{5}$.

$$
\begin{gathered}
H_{10,1}=\left[\left(\alpha_{1,1} \alpha_{1,2} \alpha_{5,1} \alpha_{5,2}\right)\left(\alpha_{2,1} \alpha_{2,2} \alpha_{4,1} \alpha_{4,2}\right)\left(\alpha_{3,1} \alpha_{3,2}\right)\left(\alpha_{1,3} \alpha_{1,4} \alpha_{5,3} \alpha_{5,4}\right)\right. \\
\left(\alpha_{2,3} \alpha_{2,4} \alpha_{4,3} \alpha_{4,4}\right)\left(\alpha_{3,3} \alpha_{3,4}\right) \\
\left(\alpha_{1,2} \alpha_{5,2}\right)\left(\alpha_{2,2} \alpha_{4,2}\right)\left(\alpha_{1,3} \alpha_{5,4}\right)\left(\alpha_{2,3} \alpha_{4,4}\right)\left(\alpha_{3,3} \alpha_{3,4}\right)\left(\alpha_{4,3} \alpha_{2,4}\right) \\
\left.\left(\alpha_{5,3} \alpha_{1,4}\right)\left(\alpha_{1,5} \alpha_{3,5}\right)\right]
\end{gathered}
$$

with orbits $\left\{\alpha_{1,1}, \alpha_{1,2}, \alpha_{5,1}, \alpha_{5,2}\right\}, \quad\left\{\alpha_{2,1}, \alpha_{2,2}, \alpha_{4,1}, \alpha_{4,2}\right\}, \quad\left\{\alpha_{3,1}, \alpha_{3,2}\right\}, \quad\left\{\alpha_{1,3}, \alpha_{1,4}, \alpha_{5,4}, \alpha_{5,3}\right\}$, $\left\{\alpha_{2,3}, \alpha_{2,4}, \alpha_{4,4}, \alpha_{4,3}\right\},\left\{\alpha_{3,3}, \alpha_{3,4}\right\},\left\{\alpha_{1,5}, \alpha_{3,5}\right\}$.

$\mathbf{n}=7, H \cong C_{6}(|H|=6, i=2)$ :

$$
\begin{gathered}
H_{7,1}=\left[\left(\alpha_{1,1} \alpha_{5,1}\right)\left(\alpha_{2,1} \alpha_{4,1}\right)\left(\alpha_{1,2} \alpha_{5,3} \alpha_{1,4} \alpha_{5,2} \alpha_{1,3} \alpha_{5,4}\right)\left(\alpha_{2,2} \alpha_{4,3} \alpha_{2,4} \alpha_{4,2} \alpha_{2,3} \alpha_{4,4}\right)\right. \\
\left.\left(\alpha_{3,2} \alpha_{3,3} \alpha_{3,4}\right)\left(\alpha_{1,5} \alpha_{3,5} \alpha_{4,5}\right)\right]
\end{gathered}
$$

with $\operatorname{Clos}\left(H_{7,1}\right)=H_{18,1}$ above.

$\mathbf{n}=6, H \cong D_{6}(|H|=6, i=1): \operatorname{rk} N_{H}=14$ and $\left(N_{H}\right)^{*} / N_{H} \cong(\mathbb{Z} / 6 \mathbb{Z})^{2} \times(\mathbb{Z} / 3 \mathbb{Z})^{3}$.

$$
\begin{gathered}
H_{6,1}=\left[\left(\alpha_{1,2} \alpha_{5,2}\right)\left(\alpha_{2,2} \alpha_{4,2}\right)\left(\alpha_{1,3} \alpha_{5,4}\right)\left(\alpha_{2,3} \alpha_{4,4}\right)\left(\alpha_{3,3} \alpha_{3,4}\right)\left(\alpha_{4,3} \alpha_{2,4}\right)\right. \\
\left(\alpha_{5,3} \alpha_{1,4}\right)\left(\alpha_{1,5} \alpha_{3,5}\right) \\
\left(\alpha_{1,2} \alpha_{1,3} \alpha_{1,4}\right)\left(\alpha_{2,2} \alpha_{2,3} \alpha_{2,4}\right)\left(\alpha_{3,2} \alpha_{3,3} \alpha_{3,4}\right)\left(\alpha_{4,2} \alpha_{4,3} \alpha_{4,4}\right) \\
\left.\left(\alpha_{5,2} \alpha_{5,3} \alpha_{5,4}\right)\left(\alpha_{1,5} \alpha_{3,5} \alpha_{4,5}\right)\right]
\end{gathered}
$$

with orbits $\left\{\alpha_{1,2}, \alpha_{5,2}, \alpha_{1,3}, \alpha_{5,3}, \alpha_{5,4}, \alpha_{1,4}\right\},\left\{\alpha_{2,2}, \alpha_{4,2}, \alpha_{2,3}, \alpha_{4,3}, \alpha_{4,4}, \alpha_{2,4}\right\},\left\{\alpha_{3,2}, \alpha_{3,3}, \alpha_{3,4}\right\}$, $\left\{\alpha_{1,5}, \alpha_{3,5}, \alpha_{4,5}\right\}$

$$
\begin{gathered}
H_{6,2}=\left[\left(\alpha_{1,1} \alpha_{5,1}\right)\left(\alpha_{2,1} \alpha_{4,1}\right)\left(\alpha_{1,3} \alpha_{1,4}\right)\left(\alpha_{2,3} \alpha_{2,4}\right)\left(\alpha_{3,3} \alpha_{3,4}\right)\left(\alpha_{4,3} \alpha_{4,4}\right)\right. \\
\left(\alpha_{5,3} \alpha_{5,4}\right)\left(\alpha_{1,5} \alpha_{3,5}\right) \\
\left(\alpha_{1,2} \alpha_{1,3} \alpha_{1,4}\right)\left(\alpha_{2,2} \alpha_{2,3} \alpha_{2,4}\right)\left(\alpha_{3,2} \alpha_{3,3} \alpha_{3,4}\right)\left(\alpha_{4,2} \alpha_{4,3} \alpha_{4,4}\right) \\
\left.\left(\alpha_{5,2} \alpha_{5,3} \alpha_{5,4}\right)\left(\alpha_{1,5} \alpha_{3,5} \alpha_{4,5}\right)\right]
\end{gathered}
$$

with orbits $\quad\left\{\alpha_{1,1}, \alpha_{5,1}\right\}, \quad\left\{\alpha_{2,1}, \alpha_{4,1}\right\}, \quad\left\{\alpha_{1,2}, \alpha_{1,3}, \alpha_{1,4}\right\}, \quad\left\{\alpha_{2,2}, \alpha_{2,3}, \alpha_{2,4}\right\}$, $\left\{\alpha_{3,2}, \alpha_{3,3}, \alpha_{3,4}\right\},\left\{\alpha_{4,2}, \alpha_{4,3}, \alpha_{4,4}\right\},\left\{\alpha_{5,2}, \alpha_{5,3}, \alpha_{5,4}\right\},\left\{\alpha_{1,5}, \alpha_{3,5}, \alpha_{4,5}\right\}$.

$\mathbf{n}=4, H \cong C_{4}(|H|=4, i=1): \operatorname{rk} N_{H}=14$ and $\left(N_{H}\right)^{*} / N_{H} \cong(\mathbb{Z} / 4 \mathbb{Z})^{4} \times(\mathbb{Z} / 2 \mathbb{Z})^{2}$.

$$
H_{4,1}=\left[\left(\alpha_{1,1} \alpha_{1,2} \alpha_{5,1} \alpha_{5,2}\right)\left(\alpha_{2,1} \alpha_{2,2} \alpha_{4,1} \alpha_{4,2}\right)\left(\alpha_{3,1} \alpha_{3,2}\right)\left(\alpha_{1,3} \alpha_{1,4} \alpha_{5,3} \alpha_{5,4}\right)\right.
$$




$$
\left.\left(\alpha_{2,3} \alpha_{2,4} \alpha_{4,3} \alpha_{4,4}\right)\left(\alpha_{3,3} \alpha_{3,4}\right)\right]
$$

with orbits $\quad\left\{\alpha_{1,1}, \alpha_{1,2}, \alpha_{5,1}, \alpha_{5,2}\right\}, \quad\left\{\alpha_{2,1}, \alpha_{2,2}, \alpha_{4,1}, \alpha_{4,2}\right\}, \quad\left\{\alpha_{3,1}, \alpha_{3,2}\right\}$, $\left\{\alpha_{1,3}, \alpha_{1,4}, \alpha_{5,3}, \alpha_{5,4}\right\},\left\{\alpha_{2,3}, \alpha_{2,4}, \alpha_{4,3}, \alpha_{4,4}\right\},\left\{\alpha_{3,3}, \alpha_{3,4}\right\}$.

$$
\mathbf{n}=\mathbf{3}, H \cong C_{2}^{2}(|H|=4, i=2): \operatorname{rk} N_{H}=12 \text { and }\left(N_{H}\right)^{*} / N_{H} \cong(\mathbb{Z} / 4 \mathbb{Z})^{2} \times(\mathbb{Z} / 2 \mathbb{Z})^{6} \text {. }
$$

$$
\begin{gathered}
H_{3,1}=\left[\left(\alpha_{1,1} \alpha_{5,1}\right)\left(\alpha_{2,1} \alpha_{4,1}\right)\left(\alpha_{1,3} \alpha_{1,4}\right)\left(\alpha_{2,3} \alpha_{2,4}\right)\left(\alpha_{3,3} \alpha_{3,4}\right)\left(\alpha_{4,3} \alpha_{4,4}\right)\right. \\
\left(\alpha_{5,3} \alpha_{5,4}\right)\left(\alpha_{1,5} \alpha_{3,5}\right), \\
\left(\alpha_{1,2} \alpha_{5,2}\right)\left(\alpha_{2,2} \alpha_{4,2}\right)\left(\alpha_{1,3} \alpha_{5,4}\right)\left(\alpha_{2,3} \alpha_{4,4}\right)\left(\alpha_{3,3} \alpha_{3,4}\right)\left(\alpha_{4,3} \alpha_{2,4}\right) \\
\left.\left(\alpha_{5,3} \alpha_{1,4}\right)\left(\alpha_{1,5} \alpha_{3,5}\right)\right]
\end{gathered}
$$

with orbits $\quad\left\{\alpha_{1,1}, \alpha_{5,1}\right\}, \quad\left\{\alpha_{2,1}, \alpha_{4,1}\right\}, \quad\left\{\alpha_{1,2}, \alpha_{5,2}\right\}, \quad\left\{\alpha_{2,2}, \alpha_{4,2}\right\}, \quad\left\{\alpha_{1,3}, \alpha_{1,4}, \alpha_{5,4}, \alpha_{5,3}\right\}$, $\left\{\alpha_{2,3}, \alpha_{2,4}, \alpha_{4,4}, \alpha_{4,3}\right\},\left\{\alpha_{3,3}, \alpha_{3,4}\right\},\left\{\alpha_{1,5}, \alpha_{3,5}\right\}$.

$\mathbf{n}=\mathbf{2}, H \cong C_{3}(|H|=3, i=1): \operatorname{rk} N_{H}=12$ and $\left(N_{H}\right)^{*} / N_{H} \cong(\mathbb{Z} / 3 \mathbb{Z})^{6}$.

$$
\begin{gathered}
H_{2,1}=\left[\left(\alpha_{1,2} \alpha_{1,3} \alpha_{1,4}\right)\left(\alpha_{2,2} \alpha_{2,3} \alpha_{2,4}\right)\left(\alpha_{3,2} \alpha_{3,3} \alpha_{3,4}\right)\left(\alpha_{4,2} \alpha_{4,3} \alpha_{4,4}\right)\right. \\
\left.\left(\alpha_{5,2} \alpha_{5,3} \alpha_{5,4}\right)\left(\alpha_{1,5} \alpha_{3,5} \alpha_{4,5}\right)\right]
\end{gathered}
$$

with orbits $\quad\left\{\alpha_{1,2}, \alpha_{1,3}, \alpha_{1,4}\right\}, \quad\left\{\alpha_{2,2}, \alpha_{2,3}, \alpha_{2,4}\right\}, \quad\left\{\alpha_{3,2}, \alpha_{3,3}, \alpha_{3,4}\right\}, \quad\left\{\alpha_{4,2}, \alpha_{4,3}, \alpha_{4,4}\right\}$, $\left\{\alpha_{5,2}, \alpha_{5,3}, \alpha_{5,4}\right\},\left\{\alpha_{1,5}, \alpha_{3,5}, \alpha_{4,5}\right\}$.

$\mathbf{n}=\mathbf{1}, H \cong C_{2}(|H|=2, i=1): \operatorname{rk} N_{H}=8$ and $\left(N_{H}\right)^{*} / N_{H} \cong(\mathbb{Z} / 2 \mathbb{Z})^{8}$.

$$
\begin{gathered}
H_{1,1}=\left[\left(\alpha_{1,2} \alpha_{5,2}\right)\left(\alpha_{2,2} \alpha_{4,2}\right)\left(\alpha_{1,3} \alpha_{5,4}\right)\left(\alpha_{2,3} \alpha_{4,4}\right)\left(\alpha_{3,3} \alpha_{3,4}\right)\left(\alpha_{4,3} \alpha_{2,4}\right)\right. \\
\left.\left(\alpha_{5,3} \alpha_{1,4}\right)\left(\alpha_{1,5}, \alpha_{3,5}\right)\right]
\end{gathered}
$$

with orbits $\left\{\alpha_{1,2}, \alpha_{5,2}\right\}, \quad\left\{\alpha_{2,2}, \alpha_{4,2}\right\}, \quad\left\{\alpha_{1,3}, \alpha_{5,4}\right\}, \quad\left\{\alpha_{2,3}, \alpha_{4,4}\right\}, \quad\left\{\alpha_{3,3}, \alpha_{3,4}\right\}, \quad\left\{\alpha_{4,3}, \alpha_{2,4}\right\}$, $\left\{\alpha_{5,3}, \alpha_{1,4}\right\},\left\{\alpha_{1,5}, \alpha_{3,5}\right\}$;

$$
\begin{gathered}
H_{1,2}=\left[\left(\alpha_{1,1} \alpha_{5,1}\right)\left(\alpha_{2,1} \alpha_{4,1}\right)\left(\alpha_{1,2} \alpha_{5,2}\right)\left(\alpha_{2,2} \alpha_{4,2}\right)\left(\alpha_{1,3} \alpha_{5,3}\right)\left(\alpha_{2,3} \alpha_{4,3}\right)\right. \\
\left.\left(\alpha_{1,4} \alpha_{5,4}\right)\left(\alpha_{2,4} \alpha_{4,4}\right)\right]
\end{gathered}
$$

with orbits $\left\{\alpha_{1,1}, \alpha_{5,1}\right\}, \quad\left\{\alpha_{2,1}, \alpha_{4,1}\right\}, \quad\left\{\alpha_{1,2}, \alpha_{5,2}\right\}, \quad\left\{\alpha_{2,2}, \alpha_{4,2}\right\}, \quad\left\{\alpha_{1,3}, \alpha_{5,3}\right\}, \quad\left\{\alpha_{2,3}, \alpha_{4,3}\right\}$, $\left\{\alpha_{1,4}, \alpha_{5,4}\right\},\left\{\alpha_{2,4}, \alpha_{4,4}\right\}$.

In [17] and [18], these conjugacy classes were described by direct considerations. Here is the correspondence between these two descriptions: $H_{54,1}=A\left(N_{18}\right) ; H_{38,1} \cong 1$ (subgroups 1 in [17] and [18]); $H_{26,1} \cong 2 ; H_{18,1} \cong 5 ; H_{14,1} \cong 3 ; H_{12,1} \cong 4 ; H_{10,1} \cong 7 ; H_{7,1} \cong 6$; 
$H_{6,1} \cong\left[\varphi_{0} \widetilde{(23)} \widetilde{(12)} \widetilde{(23)}, \widetilde{(23)}\right]$ in $8 ; H_{6,2} \cong\left[\varphi_{0} \widetilde{(23)}, \widetilde{(12)}\right]$ in $8 ; H_{4,1} \cong 9 ; H_{3,1} \cong 10 ; H_{2,1} \cong 11$; $H_{1,1} \cong[\widetilde{(i j)}]$ and $\left[\widetilde{(i j)} \varphi_{0}\right]$ in $12 ; H_{1,2} \cong\left[\varphi_{0}\right]$ in 12 .

Case 17. For the Niemeier lattice

$$
\begin{gathered}
N=N_{17}=N\left(4 A_{6}\right)= \\
=\left[4 A_{6}, \quad \varepsilon_{1,1}+2 \varepsilon_{1,2}+\varepsilon_{1,3}+6 \varepsilon_{1,4}, \quad \varepsilon_{1,1}+6 \varepsilon_{1,2}+2 \varepsilon_{1,3}+\varepsilon_{1,4}, \varepsilon_{1,1}+\varepsilon_{1,2}+6 \varepsilon_{1,3}+2 \varepsilon_{1,4}\right],
\end{gathered}
$$

the group $A(N)$ has order 24 . It is generated by

$$
\begin{gathered}
\varphi_{0}=\left(\alpha_{1,1} \alpha_{6,1}\right)\left(\alpha_{1,2} \alpha_{6,2}\right)\left(\alpha_{1,3} \alpha_{6,3}\right)\left(\alpha_{1,4} \alpha_{6,4}\right)\left(\alpha_{2,1} \alpha_{5,1}\right)\left(\alpha_{3,1} \alpha_{4,1}\right) \\
\left(\alpha_{2,2} \alpha_{5,2}\right)\left(\alpha_{3,2} \alpha_{4,2}\right)\left(\alpha_{2,3} \alpha_{5,3}\right)\left(\alpha_{3,3} \alpha_{4,3}\right)\left(\alpha_{2,4} \alpha_{5,4}\right)\left(\alpha_{3,4} \alpha_{4,4}\right), \\
\varphi_{1}=\left(\alpha_{1,2} \alpha_{1,3} \alpha_{1,4}\right)\left(\alpha_{6,2} \alpha_{6,3} \alpha_{6,4}\right)\left(\alpha_{2,2} \alpha_{2,3} \alpha_{2,4}\right)\left(\alpha_{3,2} \alpha_{3,3} \alpha_{3,4}\right) \\
\left(\alpha_{4,2} \alpha_{4,3} \alpha_{4,4}\right)\left(\alpha_{5,2} \alpha_{5,3} \alpha_{5,4}\right), \\
\varphi_{2}=\left(\alpha_{1,1} \alpha_{1,2} \alpha_{6,3}\right)\left(\alpha_{6,1} \alpha_{6,2} \alpha_{1,3}\right)\left(\alpha_{2,1} \alpha_{2,2} \alpha_{5,3}\right)\left(\alpha_{3,1} \alpha_{3,2} \alpha_{4,3}\right) \\
\left(\alpha_{4,1} \alpha_{4,2} \alpha_{3,3}\right)\left(\alpha_{5,1} \alpha_{5,2} \alpha_{2,3}\right) .
\end{gathered}
$$

\section{Classification of KahK3 conjugacy classes for $A\left(N_{17}\right)$ :}

$$
\begin{gathered}
\mathbf{n}=\mathbf{2}, H \cong C_{3}(|H|=3, i=1): \operatorname{rk} N_{H}=12 \text { and }\left(N_{H}\right)^{*} / N_{H} \cong(\mathbb{Z} / 3 \mathbb{Z})^{6} . \\
H_{2,1}=\left[\varphi_{1}=\left(\alpha_{1,2} \alpha_{1,3} \alpha_{1,4}\right)\left(\alpha_{2,2} \alpha_{2,3} \alpha_{2,4}\right)\left(\alpha_{3,2} \alpha_{3,3} \alpha_{3,4}\right)\left(\alpha_{4,2} \alpha_{4,3} \alpha_{4,4}\right)\right. \\
\left.\left(\alpha_{5,2} \alpha_{5,3} \alpha_{5,4}\right)\left(\alpha_{6,2} \alpha_{6,3} \alpha_{6,4}\right)\right]
\end{gathered}
$$

with orbits $\left\{\alpha_{1,2}, \alpha_{1,3}, \alpha_{1,4}\right\}, \quad\left\{\alpha_{2,2}, \alpha_{2,3}, \alpha_{2,4}\right\}, \quad\left\{\alpha_{3,2}, \alpha_{3,3}, \alpha_{3,4}\right\}, \quad\left\{\alpha_{4,2}, \alpha_{4,3}, \alpha_{4,4}\right\}$, $\left\{\alpha_{5,2}, \alpha_{5,3}, \alpha_{5,4}\right\},\left\{\alpha_{6,2}, \alpha_{6,3}, \alpha_{6,4}\right\}$.

In [17] and [18], the same result was obtained by direct considerations: $H_{2,1}=\left[\varphi_{1}\right] \cong C_{3}$ is the only non-trivial KahK3 conjugacy class in $A\left(N_{17}\right)$.

Case 16. For the Niemeier lattice

$$
\begin{gathered}
N=N_{16}=N\left(2 A_{7} \oplus 2 D_{5}\right)= \\
=\left[2 A_{7} \oplus 2 D_{5}, \quad \varepsilon_{1,1}+\varepsilon_{1,2}+\varepsilon_{1,3}+\varepsilon_{2,4}, \quad \varepsilon_{1,1}+7 \varepsilon_{1,2}+\varepsilon_{2,3}+\varepsilon_{1,4}\right],
\end{gathered}
$$


the group $A(N)$ has order 8 , and it is generated by

$$
\begin{gathered}
\varphi_{0}=\left(\alpha_{1,1} \alpha_{7,1}\right)\left(\alpha_{1,2} \alpha_{7,2}\right)\left(\alpha_{4,3} \alpha_{5,3}\right)\left(\alpha_{4,4} \alpha_{5,4}\right) \\
\left(\alpha_{2,1} \alpha_{6,1}\right)\left(\alpha_{3,1} \alpha_{5,1}\right)\left(\alpha_{2,2} \alpha_{6,2}\right)\left(\alpha_{3,2} \alpha_{5,2}\right), \\
\varphi_{1}=\left(\alpha_{1,1} \alpha_{1,2}\right)\left(\alpha_{7,1} \alpha_{7,2}\right)\left(\alpha_{4,4} \alpha_{5,4}\right) \\
\left(\alpha_{2,1} \alpha_{2,2}\right)\left(\alpha_{3,1} \alpha_{3,2}\right)\left(\alpha_{4,1} \alpha_{4,2}\right)\left(\alpha_{5,1} \alpha_{5,2}\right)\left(\alpha_{6,1} \alpha_{6,2}\right), \\
\varphi_{2}=\left(\alpha_{1,2} \alpha_{7,2}\right)\left(\alpha_{4,3} \alpha_{4,4}\right)\left(\alpha_{5,3} \alpha_{5,4}\right) \\
\left(\alpha_{2,2} \alpha_{6,2}\right)\left(\alpha_{3,2} \alpha_{5,2}\right)\left(\alpha_{1,3} \alpha_{1,4}\right)\left(\alpha_{2,3} \alpha_{2,4}\right)\left(\alpha_{3,3} \alpha_{3,4}\right) .
\end{gathered}
$$

(see [5, Ch. 16] and [17], [18]).

\section{Classification of KahK3 conjugacy classes for $A\left(N_{16}\right)$ :}

$$
\begin{gathered}
\mathbf{n}=\mathbf{3}, H \cong C_{2}^{2}(|H|=4, i=2): \operatorname{rk} N_{H}=12 \text { and }\left(N_{H}\right)^{*} / N_{H} \cong(\mathbb{Z} / 4 \mathbb{Z})^{2} \times(\mathbb{Z} / 2 \mathbb{Z})^{6} . \\
H_{3,1}=\left[\left(\alpha_{1,1} \alpha_{7,1}\right)\left(\alpha_{2,1} \alpha_{6,1}\right)\left(\alpha_{3,1} \alpha_{5,1}\right)\left(\alpha_{1,3} \alpha_{1,4}\right)\left(\alpha_{2,3} \alpha_{2,4}\right)\left(\alpha_{3,3} \alpha_{3,4}\right)\right. \\
\left(\alpha_{4,3} \alpha_{5,4}\right)\left(\alpha_{5,3} \alpha_{4,4}\right), \\
\left(\alpha_{1,2} \alpha_{7,2}\right)\left(\alpha_{2,2} \alpha_{6,2}\right)\left(\alpha_{3,2} \alpha_{5,2}\right)\left(\alpha_{1,3} \alpha_{1,4}\right)\left(\alpha_{2,3} \alpha_{2,4}\right)\left(\alpha_{3,3} \alpha_{3,4}\right) \\
\left.\left(\alpha_{4,3} \alpha_{4,4}\right)\left(\alpha_{5,3} \alpha_{5,4}\right)\right]
\end{gathered}
$$

with orbits $\left\{\alpha_{1,1}, \alpha_{7,1}\right\}, \quad\left\{\alpha_{2,1}, \alpha_{6,1}\right\}, \quad\left\{\alpha_{3,1}, \alpha_{5,1}\right\}, \quad\left\{\alpha_{1,2}, \alpha_{7,2}\right\}, \quad\left\{\alpha_{2,2}, \alpha_{6,2}\right\}, \quad\left\{\alpha_{3,2}, \alpha_{5,2}\right\}$, $\left\{\alpha_{1,3}, \alpha_{1,4}\right\},\left\{\alpha_{2,3}, \alpha_{2,4}\right\},\left\{\alpha_{3,3}, \alpha_{3,4}\right\},\left\{\alpha_{4,3}, \alpha_{5,4}, \alpha_{4,4}, \alpha_{5,3}\right\}$

$$
\begin{gathered}
H_{3,2}=\left[\left(\alpha_{1,1} \alpha_{7,1}\right)\left(\alpha_{2,1} \alpha_{6,1}\right)\left(\alpha_{3,1} \alpha_{5,1}\right)\left(\alpha_{1,2} \alpha_{7,2}\right)\left(\alpha_{2,2} \alpha_{6,2}\right)\left(\alpha_{3,2} \alpha_{5,2}\right)\right. \\
\left(\alpha_{4,3} \alpha_{5,3}\right)\left(\alpha_{4,4} \alpha_{5,4}\right) \\
\left(\alpha_{1,1} \alpha_{1,2}\right)\left(\alpha_{2,1} \alpha_{2,2}\right)\left(\alpha_{3,1} \alpha_{3,2}\right)\left(\alpha_{4,1} \alpha_{4,2}\right)\left(\alpha_{5,1} \alpha_{5,2}\right)\left(\alpha_{6,1} \alpha_{6,2}\right) \\
\left.\left(\alpha_{7,1} \alpha_{7,2}\right)\left(\alpha_{4,4} \alpha_{5,4}\right)\right]
\end{gathered}
$$

with orbits $\quad\left\{\alpha_{1,1}, \alpha_{7,1}, \alpha_{1,2}, \alpha_{7,2}\right\}, \quad\left\{\alpha_{2,1}, \alpha_{6,1}, \alpha_{2,2}, \alpha_{6,2}\right\}, \quad\left\{\alpha_{3,1}, \alpha_{5,1}, \alpha_{3,2}, \alpha_{5,2}\right\}$, $\left\{\alpha_{4,1}, \alpha_{4,2}\right\},\left\{\alpha_{4,3}, \alpha_{5,3}\right\},\left\{\alpha_{4,4}, \alpha_{5,4}\right\}$.

$$
\begin{gathered}
\mathbf{n}=\mathbf{1}, H \cong C_{2}(|H|=2, i=1): \operatorname{rk} N_{H}=8 \text { and }\left(N_{H}\right)^{*} / N_{H} \cong(\mathbb{Z} / 2 \mathbb{Z})^{8} . \\
H_{1,1}=\left[\left(\alpha_{1,2} \alpha_{7,2}\right)\left(\alpha_{2,2} \alpha_{6,2}\right)\left(\alpha_{3,2} \alpha_{5,2}\right)\left(\alpha_{1,3} \alpha_{1,4}\right)\left(\alpha_{2,3} \alpha_{2,4}\right)\left(\alpha_{3,3} \alpha_{3,4}\right)\right. \\
\left.\left(\alpha_{4,3} \alpha_{4,4}\right)\left(\alpha_{5,3} \alpha_{5,4}\right)\right]
\end{gathered}
$$


with orbits $\left\{\alpha_{1,2}, \alpha_{7,2}\right\}, \quad\left\{\alpha_{2,2}, \alpha_{6,2}\right\}, \quad\left\{\alpha_{3,2}, \alpha_{5,2}\right\}, \quad\left\{\alpha_{1,3}, \alpha_{1,4}\right\}, \quad\left\{\alpha_{2,3}, \alpha_{2,4}\right\}, \quad\left\{\alpha_{3,3}, \alpha_{3,4}\right\}$, $\left\{\alpha_{4,3}, \alpha_{4,4}\right\},\left\{\alpha_{5,3}, \alpha_{5,4}\right\}$

$$
\begin{gathered}
H_{1,2}=\left[\left(\alpha_{1,1} \alpha_{7,1}\right)\left(\alpha_{2,1} \alpha_{6,1}\right)\left(\alpha_{3,1} \alpha_{5,1}\right)\left(\alpha_{1,2} \alpha_{7,2}\right)\left(\alpha_{2,2} \alpha_{6,2}\right)\left(\alpha_{3,2} \alpha_{5,2}\right)\right. \\
\left.\left(\alpha_{4,3} \alpha_{5,3}\right)\left(\alpha_{4,4} \alpha_{5,4}\right)\right]
\end{gathered}
$$

with orbits $\left\{\alpha_{1,1}, \alpha_{7,1}\right\}, \quad\left\{\alpha_{2,1}, \alpha_{6,1}\right\}, \quad\left\{\alpha_{3,1}, \alpha_{5,1}\right\}, \quad\left\{\alpha_{1,2}, \alpha_{7,2}\right\}, \quad\left\{\alpha_{2,2}, \alpha_{6,2}\right\}, \quad\left\{\alpha_{3,2}, \alpha_{5,2}\right\}$, $\left\{\alpha_{4,3}, \alpha_{5,3}\right\},\left\{\alpha_{4,4}, \alpha_{5,4}\right\}$

$$
\begin{gathered}
H_{1,3}=\left[\left(\alpha_{1,1} \alpha_{1,2}\right)\left(\alpha_{2,1} \alpha_{2,2}\right)\left(\alpha_{3,1} \alpha_{3,2}\right)\left(\alpha_{4,1} \alpha_{4,2}\right)\left(\alpha_{5,1} \alpha_{5,2}\right)\left(\alpha_{6,1} \alpha_{6,2}\right)\right. \\
\left.\left(\alpha_{7,1} \alpha_{7,2}\right)\left(\alpha_{4,4} \alpha_{5,4}\right)\right]
\end{gathered}
$$

with orbits $\left\{\alpha_{1,1}, \alpha_{1,2}\right\}, \quad\left\{\alpha_{2,1}, \alpha_{2,2}\right\}, \quad\left\{\alpha_{3,1}, \alpha_{3,2}\right\}, \quad\left\{\alpha_{4,1}, \alpha_{4,2}\right\}, \quad\left\{\alpha_{5,1}, \alpha_{5,2}\right\}, \quad\left\{\alpha_{6,1}, \alpha_{6,2}\right\}$, $\left.\left\{\alpha_{7,1}, \alpha_{7,2}\right\},\left\{\alpha_{4,4}, \alpha_{5,4}\right)\right\}$.

In [17] and [18, these conjugacy classes were described by direct considerations. Here is the correspondence between these two descriptions: $H_{3,1}=\left[\varphi_{0}, \varphi_{2}\right] ; H_{3,2}=\left[\varphi_{0}, \varphi_{1}\right]$; $H_{1,1}=\left[\varphi_{2}\right] ; H_{1,2}=\left[\varphi_{0}\right] ; H_{1,3}=\left[\varphi_{1}\right]$.

Case 15. For the Niemeier lattice

$$
N=N_{15}=N\left(3 A_{8}\right)=\left[3 A_{8}, 4 \varepsilon_{1,1}+\varepsilon_{1,2}+\varepsilon_{1,3}, \quad \varepsilon_{1,1}+4 \varepsilon_{1,2}+\varepsilon_{1,3}, \quad \varepsilon_{1,1}+\varepsilon_{1,2}+4 \varepsilon_{1,3}\right]
$$

the group $A(N)$ has order 12 , and it is generated by

$$
\begin{gathered}
\varphi_{0}=\left(\alpha_{1,1} \alpha_{8,1}\right)\left(\alpha_{1,2} \alpha_{8,2}\right)\left(\alpha_{1,3} \alpha_{8,3}\right) \\
\left(\alpha_{2,1} \alpha_{7,1}\right)\left(\alpha_{3,1} \alpha_{6,1}\right)\left(\alpha_{4,1} \alpha_{5,1}\right)\left(\alpha_{2,2} \alpha_{7,2}\right)\left(\alpha_{3,2} \alpha_{6,2}\right)\left(\alpha_{4,2} \alpha_{5,2}\right) \\
\left(\alpha_{2,3} \alpha_{7,3}\right)\left(\alpha_{3,3} \alpha_{6,3}\right)\left(\alpha_{4,3} \alpha_{5,3}\right), \\
\widetilde{(12)}=\left(\alpha_{1,1} \alpha_{1,2}\right)\left(\alpha_{8,1} \alpha_{8,2}\right) \\
\left(\alpha_{2,1} \alpha_{2,2}\right)\left(\alpha_{3,1} \alpha_{3,2}\right)\left(\alpha_{4,1} \alpha_{4,2}\right)\left(\alpha_{5,1} \alpha_{5,2}\right) \\
\left(\alpha_{6,1} \alpha_{6,2}\right)\left(\alpha_{7,1} \alpha_{7,2}\right), \\
\widetilde{(23)}=\left(\alpha_{1,2} \alpha_{1,3}\right)\left(\alpha_{8,2} \alpha_{8,3}\right) \\
\left(\alpha_{2,2} \alpha_{2,3}\right)\left(\alpha_{3,2} \alpha_{3,3}\right)\left(\alpha_{4,2} \alpha_{4,3}\right)\left(\alpha_{5,2} \alpha_{5,3}\right) \\
\left(\alpha_{6,2} \alpha_{6,3}\right)\left(\alpha_{7,2} \alpha_{7,3}\right) .
\end{gathered}
$$

(see [5, Ch. 16] and [17], [18]). 


\section{Classification of KahK3 conjugacy classes for $A\left(N_{15}\right)$ :}

$\mathbf{n}=\mathbf{1}, H \cong C_{2}(|H|=2, i=1): \operatorname{rk} N_{H}=8$ and $\left(N_{H}\right)^{*} / N_{H} \cong(\mathbb{Z} / 2 \mathbb{Z})^{8}$.

$$
\begin{gathered}
H_{1,1}=\left[\widetilde{(23)}=\left(\alpha_{1,2} \alpha_{1,3}\right)\left(\alpha_{2,2} \alpha_{2,3}\right)\left(\alpha_{3,2} \alpha_{3,3}\right)\left(\alpha_{4,2} \alpha_{4,3}\right)\left(\alpha_{5,2} \alpha_{5,3}\right)\left(\alpha_{6,2} \alpha_{6,3}\right)\right. \\
\left.\left(\alpha_{7,2} \alpha_{7,3}\right)\left(\alpha_{8,2} \alpha_{8,3}\right)\right]
\end{gathered}
$$

with orbits $\left\{\alpha_{1,2}, \alpha_{1,3}\right\}, \quad\left\{\alpha_{2,2}, \alpha_{2,3}\right\}, \quad\left\{\alpha_{3,2}, \alpha_{3,3}\right\}, \quad\left\{\alpha_{4,2}, \alpha_{4,3}\right\}, \quad\left\{\alpha_{5,2}, \alpha_{5,3}\right\}, \quad\left\{\alpha_{6,2}, \alpha_{6,3}\right\}$, $\left\{\alpha_{7,2}, \alpha_{7,3}\right\},\left\{\alpha_{8,2}, \alpha_{8,3}\right\}$.

In [17] and [18], the conjugacy class of $H_{1,1}=[\widetilde{(23)}]$ was described by direct considerations.

Case 14. For the Niemeier lattice

$$
\begin{gathered}
N=N_{14}=N\left(4 D_{6}\right)=\left[4 D_{6}, \text { even permutations of } 0_{1}+\varepsilon_{1,2}+\varepsilon_{2,3}+\varepsilon_{3,4}\right] \\
=\left[4 D_{6}, \quad \varepsilon_{1,2}+\varepsilon_{2,3}+\varepsilon_{3,4}, \quad \varepsilon_{3,2}+\varepsilon_{1,3}+\varepsilon_{2,4}, \quad \varepsilon_{2,2}+\varepsilon_{3,3}+\varepsilon_{1,4}\right. \\
\varepsilon_{1,1}+\varepsilon_{3,3}+\varepsilon_{2,4}, \quad \varepsilon_{2,1}+\varepsilon_{1,3}+\varepsilon_{3,4}, \quad \varepsilon_{3,1}+\varepsilon_{2,3}+\varepsilon_{1,4} \\
\varepsilon_{2,1}+\varepsilon_{3,2}+\varepsilon_{1,4}, \quad \varepsilon_{1,1}+\varepsilon_{2,2}+\varepsilon_{3,4}, \quad \varepsilon_{3,1}+\varepsilon_{1,2}+\varepsilon_{2,4} \\
\left.\varepsilon_{3,1}+\varepsilon_{2,2}+\varepsilon_{1,3}, \quad \varepsilon_{1,1}+\varepsilon_{3,2}+\varepsilon_{2,3}, \quad \varepsilon_{2,1}+\varepsilon_{1,2}+\varepsilon_{3,3}\right]
\end{gathered}
$$

the group $A(N)$ has order 24 , and it is generated by

$$
\begin{gathered}
(12)=\left(\alpha_{1,1} \alpha_{1,2}\right)\left(\alpha_{5,1} \alpha_{6,2}\right)\left(\alpha_{6,1} \alpha_{5,2}\right)\left(\alpha_{5,3} \alpha_{6,3}\right)\left(\alpha_{5,4} \alpha_{6,4}\right) \\
\left(\alpha_{2,1} \alpha_{2,2}\right)\left(\alpha_{3,1} \alpha_{3,2}\right)\left(\alpha_{4,1} \alpha_{4,2}\right), \\
(23)=\left(\alpha_{1,2} \alpha_{1,3}\right)\left(\alpha_{5,2} \alpha_{6,3}\right)\left(\alpha_{6,2} \alpha_{5,3}\right)\left(\alpha_{5,1} \alpha_{6,1}\right)\left(\alpha_{5,4} \alpha_{6,4}\right) \\
\left(\alpha_{2,2} \alpha_{2,3}\right)\left(\alpha_{3,2} \alpha_{3,3}\right)\left(\alpha_{4,2} \alpha_{4,3}\right), \\
(34)=\left(\alpha_{1,3} \alpha_{1,4}\right)\left(\alpha_{5,3} \alpha_{6,4}\right)\left(\alpha_{6,3} \alpha_{5,4}\right)\left(\alpha_{5,1} \alpha_{6,1}\right)\left(\alpha_{5,2} \alpha_{6,2}\right) \\
\left(\alpha_{2,3} \alpha_{2,4}\right)\left(\alpha_{3,3} \alpha_{3,4}\right)\left(\alpha_{4,3} \alpha_{4,4}\right)
\end{gathered}
$$

(see [5, Ch. 16] and [17], [18]).

\section{Classification of KahK3 conjugacy classes for $A\left(N_{14}\right)$ :}

$$
\begin{gathered}
\mathbf{n}=\mathbf{6}, H \cong D_{6}(|H|=6, i=1): \operatorname{rk} N_{H}=14 \text { and }\left(N_{H}\right)^{*} / N_{H} \cong(\mathbb{Z} / 6 \mathbb{Z})^{2} \times(\mathbb{Z} / 3 \mathbb{Z})^{3} . \\
H_{6,1}=\left[\left(\alpha_{5,1} \alpha_{6,1}\right)\left(\alpha_{5,2} \alpha_{6,2}\right)\left(\alpha_{1,3} \alpha_{1,4}\right)\left(\alpha_{2,3} \alpha_{2,4}\right)\left(\alpha_{3,3} \alpha_{3,4}\right)\left(\alpha_{4,3} \alpha_{4,4}\right)\right.
\end{gathered}
$$




$$
\begin{gathered}
\left(\alpha_{5,3} \alpha_{6,4}\right)\left(\alpha_{6,3} \alpha_{5,4}\right), \\
\left(\alpha_{1,2} \alpha_{1,3} \alpha_{1,4}\right)\left(\alpha_{2,2} \alpha_{2,3} \alpha_{2,4}\right)\left(\alpha_{3,2} \alpha_{3,3} \alpha_{3,4}\right)\left(\alpha_{4,2} \alpha_{4,3} \alpha_{4,4}\right) \\
\left.\left(\alpha_{5,2} \alpha_{5,3} \alpha_{5,4}\right)\left(\alpha_{6,2} \alpha_{6,3} \alpha_{6,4}\right)\right]
\end{gathered}
$$

with orbits $\quad\left\{\alpha_{5,1}, \alpha_{6,1}\right\}, \quad\left\{\alpha_{1,2}, \alpha_{1,3}, \alpha_{1,4}\right\}, \quad\left\{\alpha_{2,2}, \alpha_{2,3}, \alpha_{2,4}\right\}, \quad\left\{\alpha_{3,2}, \alpha_{3,3}, \alpha_{3,4}\right\}$, $\left\{\alpha_{4,2}, \alpha_{4,3}, \alpha_{4,4}\right\},\left\{\alpha_{5,2}, \alpha_{6,2}, \alpha_{5,3}, \alpha_{6,3}, \alpha_{6,4}, \alpha_{5,4}\right\}$.

$$
\mathbf{n}=\mathbf{2}, H \cong C_{3}(|H|=3, i=1): \operatorname{rk} N_{H}=12 \text { and }\left(N_{H}\right)^{*} / N_{H} \cong(\mathbb{Z} / 3 \mathbb{Z})^{6} \text {. }
$$

$$
\begin{gathered}
H_{2,1}=\left[\left(\alpha_{1,2} \alpha_{1,3} \alpha_{1,4}\right)\left(\alpha_{2,2} \alpha_{2,3} \alpha_{2,4}\right)\left(\alpha_{3,2} \alpha_{3,3} \alpha_{3,4}\right)\left(\alpha_{4,2} \alpha_{4,3} \alpha_{4,4}\right)\right. \\
\left.\left(\alpha_{5,2} \alpha_{5,3} \alpha_{5,4}\right)\left(\alpha_{6,2} \alpha_{6,3} \alpha_{6,4}\right)\right]
\end{gathered}
$$

with orbits $\left\{\alpha_{1,2}, \alpha_{1,3}, \alpha_{1,4}\right\}, \quad\left\{\alpha_{2,2}, \alpha_{2,3}, \alpha_{2,4}\right\}, \quad\left\{\alpha_{3,2}, \alpha_{3,3}, \alpha_{3,4}\right\}, \quad\left\{\alpha_{4,2}, \alpha_{4,3}, \alpha_{4,4}\right\}$, $\left\{\alpha_{5,2}, \alpha_{5,3}, \alpha_{5,4}\right\},\left\{\alpha_{6,2}, \alpha_{6,3}, \alpha_{6,4}\right\}$.

$$
\mathbf{n}=\mathbf{1}, H \cong C_{2}(|H|=2, i=1): \operatorname{rk} N_{H}=8 \text { and }\left(N_{H}\right)^{*} / N_{H} \cong(\mathbb{Z} / 2 \mathbb{Z})^{8} \text {. }
$$

$$
\begin{gathered}
H_{1,1}=\left[\left(\alpha_{5,1} \alpha_{6,1}\right)\left(\alpha_{5,2} \alpha_{6,2}\right)\left(\alpha_{1,3} \alpha_{1,4}\right)\left(\alpha_{2,3} \alpha_{2,4}\right)\left(\alpha_{3,3} \alpha_{3,4}\right)\left(\alpha_{4,3} \alpha_{4,4}\right)\right. \\
\left.\left(\alpha_{5,3} \alpha_{6,4}\right)\left(\alpha_{6,3} \alpha_{5,4}\right)\right]
\end{gathered}
$$

with orbits $\left\{\alpha_{5,1}, \alpha_{6,1}\right\}, \quad\left\{\alpha_{5,2}, \alpha_{6,2}\right\}, \quad\left\{\alpha_{1,3}, \alpha_{1,4}\right\}, \quad\left\{\alpha_{2,3}, \alpha_{2,4}\right\}, \quad\left\{\alpha_{3,3}, \alpha_{3,4}\right\}, \quad\left\{\alpha_{4,3}, \alpha_{4,4}\right\}$, $\left\{\alpha_{5,3}, \alpha_{6,4}\right\},\left\{\alpha_{6,3}, \alpha_{5,4}\right\}$.

In [17] and [18], these conjugacy classes were described by direct considerations. Here is the correspondence between these two descriptions: $H_{6,1}=\left(\mathfrak{D}_{6}\right)_{1} ; H_{2,1}=\left(C_{3}\right)_{1} ; H_{1,1}=$ $[(34)]$.

Case 13. For the Niemeier lattice

$$
N=N_{13}=N\left(2 A_{9} \oplus D_{6}\right)=\left[2 A_{9} \oplus D_{6}, 2 \varepsilon_{1,1}+4 \varepsilon_{1,2}, 5 \varepsilon_{1,1}+\varepsilon_{1,3}, 5 \varepsilon_{1,2}+\varepsilon_{3,3}\right]
$$

the group $A(N)$ has order 4 , and it is generated by

$$
\begin{gathered}
\varphi=\left(\alpha_{1,1} \alpha_{1,2} \alpha_{9,1} \alpha_{9,2}\right)\left(\alpha_{5,3} \alpha_{6,3}\right) \\
\left(\alpha_{2,1} \alpha_{2,2} \alpha_{8,1} \alpha_{8,2}\right)\left(\alpha_{3,1} \alpha_{3,2} \alpha_{7,1} \alpha_{7,2}\right)\left(\alpha_{4,1} \alpha_{4,2} \alpha_{6,1} \alpha_{6,2}\right)\left(\alpha_{5,1} \alpha_{5,2}\right)
\end{gathered}
$$

(see [5, Ch. 16] and [17], [18]).

\section{Classification of KahK3 conjugacy classes for $A\left(N_{13}\right)$ :}


$\mathbf{n}=4, H \cong C_{4}(|H|=4, i=1): \operatorname{rk} N_{H}=14$ and $\left(N_{H}\right)^{*} / N_{H} \cong(\mathbb{Z} / 4 \mathbb{Z})^{4} \times(\mathbb{Z} / 2 \mathbb{Z})^{2}$.

$$
\begin{gathered}
H_{4,1}=\left[\varphi=\left(\alpha_{1,1} \alpha_{1,2} \alpha_{9,1} \alpha_{9,2}\right)\left(\alpha_{5,3} \alpha_{6,3}\right)\right. \\
\left(\alpha_{2,1} \alpha_{2,2} \alpha_{8,1} \alpha_{8,2}\right)\left(\alpha_{3,1} \alpha_{3,2} \alpha_{7,1} \alpha_{7,2}\right)\left(\alpha_{4,1} \alpha_{4,2} \alpha_{6,1} \alpha_{6,2}\right)\left(\alpha_{5,1} \alpha_{5,2}\right)
\end{gathered}
$$

with orbits $\quad\left\{\alpha_{1,1}, \alpha_{1,2}, \alpha_{9,1}, \alpha_{9,2}\right\}, \quad\left\{\alpha_{2,1}, \alpha_{2,2}, \alpha_{8,1}, \alpha_{8,2}\right\}, \quad\left\{\alpha_{3,1}, \alpha_{3,2}, \alpha_{7,1}, \alpha_{7,2}\right\}$, $\left\{\alpha_{4,1}, \alpha_{4,2}, \alpha_{6,1}, \alpha_{6,2}\right\},\left\{\alpha_{5,1}, \alpha_{5,2}\right\},\left\{\alpha_{5,3}, \alpha_{6,3}\right\}$.

$\mathbf{n}=\mathbf{1}, H \cong C_{2}(|H|=2, i=1): \operatorname{rk} N_{H}=8$ and $\left(N_{H}\right)^{*} / N_{H} \cong(\mathbb{Z} / 2 \mathbb{Z})^{8}$.

$$
\begin{gathered}
H_{1,1}=\left[\varphi^{2}=\left(\alpha_{1,1} \alpha_{9,1}\right)\left(\alpha_{1,2} \alpha_{9,2}\right)\left(\alpha_{2,1} \alpha_{8,1}\right)\left(\alpha_{3,1} \alpha_{7,1}\right)\left(\alpha_{4,1} \alpha_{6,1}\right)\right. \\
\left.\left(\alpha_{2,2} \alpha_{8,2}\right)\left(\alpha_{3,2} \alpha_{7,2}\right)\left(\alpha_{4,2} \alpha_{6,2}\right)\right]
\end{gathered}
$$

with orbits $\left\{\alpha_{1,1}, \alpha_{9,1}\right\}, \quad\left\{\alpha_{1,2}, \alpha_{9,2}\right\}, \quad\left\{\alpha_{2,1}, \alpha_{8,1},\right\}, \quad\left\{\alpha_{3,1}, \alpha_{7,1}\right\}, \quad\left\{\alpha_{4,1}, \alpha_{6,1}\right\}, \quad\left\{\alpha_{2,2}, \alpha_{8,2}\right\}$, $\left\{\alpha_{3,2}, \alpha_{7,2}\right\},\left\{\alpha_{4,2}, \alpha_{6,2}\right\}$.

Case 12. For the Niemeier lattice

$$
\begin{gathered}
N_{12}=N\left(4 E_{6}\right)=\left[4 E_{6}, \quad \varepsilon_{1,1}+\varepsilon_{1,3}+\varepsilon_{2,4}, \quad \varepsilon_{1,1}+\varepsilon_{2,2}+\varepsilon_{1,4},\right. \\
\left.\varepsilon_{1,1}+\varepsilon_{1,2}+\varepsilon_{2,3}\right],
\end{gathered}
$$

the group $A(N)$ has order 48 , and it is generated by

$$
\begin{gathered}
\varphi_{0}=\left(\alpha_{1,1} \alpha_{6,1}\right)\left(\alpha_{1,2} \alpha_{6,2}\right)\left(\alpha_{1,3} \alpha_{6,3}\right)\left(\alpha_{1,4} \alpha_{6,4}\right) \\
\left(\alpha_{3,1} \alpha_{5,1}\right)\left(\alpha_{3,2} \alpha_{5,2}\right)\left(\alpha_{3,3} \alpha_{5,3}\right)\left(\alpha_{3,4} \alpha_{5,4}\right), \\
\widetilde{(12)}=\left(\alpha_{1,1} \alpha_{1,2}\right)\left(\alpha_{6,1} \alpha_{6,2}\right)\left(\alpha_{1,4} \alpha_{6,4}\right) \\
\left(\alpha_{2,1} \alpha_{2,2}\right)\left(\alpha_{3,1} \alpha_{3,2}\right)\left(\alpha_{4,1} \alpha_{4,2}\right)\left(\alpha_{5,1} \alpha_{5,2}\right)\left(\alpha_{3,4}, \alpha_{5,4}\right), \\
\widetilde{(23)}=\left(\alpha_{1,1} \alpha_{6,1}\right)\left(\alpha_{1,2} \alpha_{1,3}\right)\left(\alpha_{6,2} \alpha_{6,3}\right) \\
\left(\alpha_{3,1} \alpha_{5,1}\right)\left(\alpha_{2,2} \alpha_{2,3}\right)\left(\alpha_{3,2} \alpha_{3,3}\right)\left(\alpha_{4,2} \alpha_{4,3}\right)\left(\alpha_{5,2} \alpha_{5,3}\right), \\
\widetilde{(34)}=\left(\alpha_{1,1} \alpha_{6,1}\right)\left(\alpha_{1,3} \alpha_{1,4}\right)\left(\alpha_{6,3} \alpha_{6,4}\right) \\
\left(\alpha_{3,1} \alpha_{5,1}\right)\left(\alpha_{2,3} \alpha_{2,4}\right)\left(\alpha_{3,3} \alpha_{3,4}\right)\left(\alpha_{4,3} \alpha_{4,4}\right)\left(\alpha_{5,3} \alpha_{5,4}\right) .
\end{gathered}
$$

Classification of KahK3 conjugacy classes for $A\left(N_{12}\right)$ : 
$\mathbf{n}=\mathbf{1 8}, H \cong D_{12}(|H|=12, i=4): \operatorname{rk} N_{H}=16$ and $\left(N_{H}\right)^{*} / N_{H} \cong(\mathbb{Z} / 6 \mathbb{Z})^{4}$.

$$
\begin{gathered}
H_{18,1}=\left[\left(\alpha_{1,2} \alpha_{6,2}\right)\left(\alpha_{3,2} \alpha_{5,2}\right)\left(\alpha_{1,3} \alpha_{6,4}\right)\left(\alpha_{2,3} \alpha_{2,4}\right)\left(\alpha_{3,3} \alpha_{5,4}\right)\left(\alpha_{4,3} \alpha_{4,4}\right)\right. \\
\left(\alpha_{5,3} \alpha_{3,4}\right)\left(\alpha_{6,3} \alpha_{1,4}\right), \\
\left(\alpha_{1,1} \alpha_{6,1}\right)\left(\alpha_{3,1} \alpha_{5,1}\right)\left(\alpha_{1,2} \alpha_{6,3} \alpha_{1,4} \alpha_{6,2} \alpha_{1,3} \alpha_{6,4}\right)\left(\alpha_{2,2} \alpha_{2,3} \alpha_{2,4}\right) \\
\left.\left(\alpha_{3,2} \alpha_{5,3} \alpha_{3,4} \alpha_{5,2} \alpha_{3,3} \alpha_{5,4}\right)\left(\alpha_{4,2} \alpha_{4,3} \alpha_{4,4}\right)\right]
\end{gathered}
$$

with orbits $\left\{\alpha_{1,1}, \alpha_{6,1}\right\}, \quad\left\{\alpha_{3,1}, \alpha_{5,1}\right\}, \quad\left\{\alpha_{1,2}, \alpha_{6,2}, \alpha_{6,3}, \alpha_{1,3}, \alpha_{1,4}, \alpha_{6,4}\right\}, \quad\left\{\alpha_{2,2}, \alpha_{2,3}, \alpha_{2,4}\right\}$, $\left\{\alpha_{3,2}, \alpha_{5,2}, \alpha_{5,3}, \alpha_{3,3}, \alpha_{3,4}, \alpha_{5,4}\right\},\left\{\alpha_{4,2}, \alpha_{4,3}, \alpha_{4,4}\right\}$.

$\mathbf{n}=7, H \cong C_{6}(|H|=6, i=2)$ :

$$
\begin{gathered}
H_{7,1}=\left[\left(\alpha_{1,1} \alpha_{6,1}\right)\left(\alpha_{3,1} \alpha_{5,1}\right)\left(\alpha_{1,2} \alpha_{6,3} \alpha_{1,4} \alpha_{6,2} \alpha_{1,3} \alpha_{6,4}\right)\left(\alpha_{2,2} \alpha_{2,3} \alpha_{2,4}\right)\right. \\
\left.\left(\alpha_{3,2} \alpha_{5,3} \alpha_{3,4} \alpha_{5,2} \alpha_{3,3} \alpha_{5,4}\right)\left(\alpha_{4,2} \alpha_{4,3} \alpha_{4,4}\right)\right]
\end{gathered}
$$

has $\operatorname{Clos}\left(H_{7,1}\right)=H_{18,1}$ above.

$\mathbf{n}=6, H \cong D_{6}(|H|=6, i=1): \operatorname{rk} N_{H}=14$ and $\left(N_{H}\right)^{*} / N_{H} \cong(\mathbb{Z} / 6 \mathbb{Z})^{2} \times(\mathbb{Z} / 3 \mathbb{Z})^{3}$.

$$
\begin{gathered}
H_{6,1}=\left[\left(\alpha_{1,2} \alpha_{6,2}\right)\left(\alpha_{3,2} \alpha_{5,2}\right)\left(\alpha_{1,3} \alpha_{6,4}\right)\left(\alpha_{2,3} \alpha_{2,4}\right)\left(\alpha_{3,3} \alpha_{5,4}\right)\left(\alpha_{4,3} \alpha_{4,4}\right)\right. \\
\left(\alpha_{5,3} \alpha_{3,4}\right)\left(\alpha_{6,3} \alpha_{1,4}\right) \\
\left(\alpha_{1,2} \alpha_{1,3} \alpha_{1,4}\right)\left(\alpha_{2,2} \alpha_{2,3} \alpha_{2,4}\right)\left(\alpha_{3,2} \alpha_{3,3} \alpha_{3,4}\right)\left(\alpha_{4,2} \alpha_{4,3} \alpha_{4,4}\right) \\
\left.\left(\alpha_{5,2} \alpha_{5,3} \alpha_{5,4}\right)\left(\alpha_{6,2} \alpha_{6,3} \alpha_{6,4}\right)\right]
\end{gathered}
$$

with orbits $\left\{\alpha_{1,2}, \alpha_{6,2}, \alpha_{1,3}, \alpha_{6,3}, \alpha_{6,4}, \alpha_{1,4}\right\},\left\{\alpha_{2,2}, \alpha_{2,3}, \alpha_{2,4}\right\},\left\{\alpha_{3,2}, \alpha_{5,2}, \alpha_{3,3}, \alpha_{5,3}, \alpha_{5,4}, \alpha_{3,4}\right\}$, $\left\{\alpha_{4,2}, \alpha_{4,3}, \alpha_{4,4}\right\}$

$$
\begin{gathered}
H_{6,2}=\left[\left(\alpha_{1,1} \alpha_{6,1}\right)\left(\alpha_{3,1} \alpha_{5,1}\right)\left(\alpha_{1,3} \alpha_{1,4}\right)\left(\alpha_{2,3} \alpha_{2,4}\right)\left(\alpha_{3,3} \alpha_{3,4}\right)\left(\alpha_{4,3} \alpha_{4,4}\right)\right. \\
\left(\alpha_{5,3} \alpha_{5,4}\right)\left(\alpha_{6,3} \alpha_{6,4}\right), \\
\left(\alpha_{1,2} \alpha_{1,3} \alpha_{1,4}\right)\left(\alpha_{2,2} \alpha_{2,3} \alpha_{2,4}\right)\left(\alpha_{3,2} \alpha_{3,3} \alpha_{3,4}\right)\left(\alpha_{4,2} \alpha_{4,3} \alpha_{4,4}\right) \\
\left.\left(\alpha_{5,2} \alpha_{5,3} \alpha_{5,4}\right)\left(\alpha_{6,2} \alpha_{6,3} \alpha_{6,4}\right)\right]
\end{gathered}
$$

with orbits $\left\{\alpha_{1,1}, \alpha_{6,1}\right\}, \quad\left\{\alpha_{3,1}, \alpha_{5,1}\right\}, \quad\left\{\alpha_{1,2}, \alpha_{1,3}, \alpha_{1,4}\right\}, \quad\left\{\alpha_{2,2}, \alpha_{2,3}, \alpha_{2,4}\right\}, \quad\left\{\alpha_{3,2}, \alpha_{3,3}, \alpha_{3,4}\right\}$, $\left\{\alpha_{4,2}, \alpha_{4,3}, \alpha_{4,4}\right\},\left\{\alpha_{5,2}, \alpha_{5,3}, \alpha_{5,4}\right\},\left\{\alpha_{6,2}, \alpha_{6,3}, \alpha_{6,4}\right\}$.

$\mathbf{n}=\mathbf{3}, H \cong C_{2}^{2}(|H|=4, i=2): \operatorname{rk} N_{H}=12$ and $\left(N_{H}\right)^{*} / N_{H} \cong(\mathbb{Z} / 4 \mathbb{Z})^{2} \times(\mathbb{Z} / 2 \mathbb{Z})^{6}$.

$$
H_{3,1}=\left[\varphi_{0}=\left(\alpha_{1,1} \alpha_{6,1}\right)\left(\alpha_{1,2} \alpha_{6,2}\right)\left(\alpha_{1,3} \alpha_{6,3}\right)\left(\alpha_{1,4} \alpha_{6,4}\right)\right.
$$




$$
\begin{gathered}
\left(\alpha_{3,1} \alpha_{5,1}\right)\left(\alpha_{3,2} \alpha_{5,2}\right)\left(\alpha_{3,3} \alpha_{5,3}\right)\left(\alpha_{3,4} \alpha_{5,4}\right), \\
\widetilde{(34)}=\left(\alpha_{1,1} \alpha_{6,1}\right)\left(\alpha_{1,3} \alpha_{1,4}\right)\left(\alpha_{6,3} \alpha_{6,4}\right) \\
\left.\left(\alpha_{3,1} \alpha_{5,1}\right)\left(\alpha_{2,3} \alpha_{2,4}\right)\left(\alpha_{3,3} \alpha_{3,4}\right)\left(\alpha_{4,3} \alpha_{4,4}\right)\left(\alpha_{5,3} \alpha_{5,4}\right)\right]
\end{gathered}
$$

with orbits $\left\{\alpha_{1,1}, \alpha_{6,1}\right\}, \quad\left\{\alpha_{3,1}, \alpha_{5,1}\right\}, \quad\left\{\alpha_{1,2}, \alpha_{6,2}\right\}, \quad\left\{\alpha_{3,2}, \alpha_{5,2}\right\}, \quad\left\{\alpha_{1,3}, \alpha_{6,3}, \alpha_{6,4}, \alpha_{1,4}\right\}$, $\left\{\alpha_{2,3}, \alpha_{2,4}\right\},\left\{\alpha_{3,3}, \alpha_{5,3}, \alpha_{5,4}, \alpha_{3,4}\right\},\left\{\alpha_{4,3}, \alpha_{4,4}\right\}$.

$\mathbf{n}=\mathbf{2}, H \cong C_{3}(|H|=3, i=1): \operatorname{rk} N_{H}=12$ and $\left(N_{H}\right)^{*} / N_{H} \cong(\mathbb{Z} / 3 \mathbb{Z})^{6}$.

$$
\begin{gathered}
H_{2,1}=\left[\left(\alpha_{1,2} \alpha_{1,3} \alpha_{1,4}\right)\left(\alpha_{2,2} \alpha_{2,3} \alpha_{2,4}\right)\left(\alpha_{3,2} \alpha_{3,3} \alpha_{3,4}\right)\left(\alpha_{4,2} \alpha_{4,3} \alpha_{4,4}\right)\right. \\
\left.\left(\alpha_{5,2} \alpha_{5,3} \alpha_{5,4}\right)\left(\alpha_{6,2} \alpha_{6,3} \alpha_{6,4}\right)\right]
\end{gathered}
$$

with orbits $\quad\left\{\alpha_{1,2}, \alpha_{1,3}, \alpha_{1,4}\right\}, \quad\left\{\alpha_{2,2}, \alpha_{2,3}, \alpha_{2,4}\right\}, \quad\left\{\alpha_{3,2}, \alpha_{3,3}, \alpha_{3,4}\right\}, \quad\left\{\alpha_{4,2}, \alpha_{4,3}, \alpha_{4,4}\right\}$, $\left\{\alpha_{5,2}, \alpha_{5,3}, \alpha_{5,4}\right\},\left\{\alpha_{6,2}, \alpha_{6,3}, \alpha_{6,4}\right\}$.

$\mathbf{n}=\mathbf{1}, H \cong C_{2}(|H|=2, i=1): \operatorname{rk} N_{H}=8$ and $\left(N_{H}\right)^{*} / N_{H} \cong(\mathbb{Z} / 2 \mathbb{Z})^{8}$.

$$
\begin{aligned}
H_{1,1}= & {\left[\varphi_{0}=\left(\alpha_{1,1} \alpha_{6,1}\right)\left(\alpha_{1,2} \alpha_{6,2}\right)\left(\alpha_{1,3} \alpha_{6,3}\right)\left(\alpha_{1,4} \alpha_{6,4}\right)\right.} \\
& \left.\left(\alpha_{3,1} \alpha_{5,1}\right)\left(\alpha_{3,2} \alpha_{5,2}\right)\left(\alpha_{3,3} \alpha_{5,3}\right)\left(\alpha_{3,4} \alpha_{5,4}\right)\right],
\end{aligned}
$$

with orbits $\left\{\alpha_{1,1}, \alpha_{6,1}\right\}, \quad\left\{\alpha_{1,2}, \alpha_{6,2}\right\}, \quad\left\{\alpha_{1,3}, \alpha_{6,3}\right\}, \quad\left\{\alpha_{1,4}, \alpha_{6,4}\right\}, \quad\left\{\alpha_{3,1}, \alpha_{5,1}\right\}, \quad\left\{\alpha_{3,2}, \alpha_{5,2}\right\}$, $\left\{\alpha_{3,3}, \alpha_{5,3}\right\},\left\{\alpha_{3,4}, \alpha_{5,4}\right\}$;

$$
\begin{gathered}
H_{1,2}=\left[\widetilde{(34)}=\left(\alpha_{1,1} \alpha_{6,1}\right)\left(\alpha_{1,3} \alpha_{1,4}\right)\left(\alpha_{6,3} \alpha_{6,4}\right)\right. \\
\left.\left(\alpha_{3,1} \alpha_{5,1}\right)\left(\alpha_{2,3} \alpha_{2,4}\right)\left(\alpha_{3,3} \alpha_{3,4}\right)\left(\alpha_{4,3} \alpha_{4,4}\right)\left(\alpha_{5,3} \alpha_{5,4}\right)\right]
\end{gathered}
$$

with orbits $\left\{\alpha_{1,1}, \alpha_{6,1}\right\}, \quad\left\{\alpha_{1,3}, \alpha_{1,4}\right\}, \quad\left\{\alpha_{6,3}, \alpha_{6,4}\right\}, \quad\left\{\alpha_{3,1}, \alpha_{5,1}\right\}, \quad\left\{\alpha_{2,3}, \alpha_{2,4}\right\}, \quad\left\{\alpha_{3,3}, \alpha_{3,4}\right\}$, $\left\{\alpha_{4,3}, \alpha_{4,4}\right\},\left\{\alpha_{5,3}, \alpha_{5,4}\right\}$.

In [17] and [18], these conjugacy classes were described by direct considerations. Here is the correspondence between these two descriptions: $H_{12,1}=\left(\mathfrak{D}_{12}\right)_{1} ; H_{7,1}=\left(C_{6}\right)_{1}$; $\left\{H_{6,1}, H_{6,2}\right\}=\left\{\left(\mathfrak{D}_{6}\right)_{11},\left(\mathfrak{D}_{6}\right)_{12}\right\} ; H_{3,1}=\left[\varphi_{0}, \widetilde{(34)}\right] ; H_{2,1}=\left(C_{3}\right)_{1} ; H_{1,1}=\left[\varphi_{0}\right] ; H_{1,2}=[\widetilde{(34)}]$.

Case 11. For the Niemeier lattice $N_{11}=N\left(A_{11} \oplus D_{7} \oplus E_{6}\right)=\left[A_{11} \oplus D_{7} \oplus E_{6}, \varepsilon_{1,1}+\right.$ $\left.\varepsilon_{1,2}+\varepsilon_{1,3}\right]$, the group $A\left(N_{11}\right)$ has order 2 , and it is generated by

$$
\begin{gathered}
\varphi=\left(\alpha_{1,1} \alpha_{11,1}\right)\left(\alpha_{2,1} \alpha_{10,1}\right)\left(\alpha_{3,1} \alpha_{9,1}\right)\left(\alpha_{4,1} \alpha_{8,1}\right)\left(\alpha_{5,1} \alpha_{7,1}\right)\left(\alpha_{6,2} \alpha_{7,2}\right) \\
\left(\alpha_{1,3} \alpha_{6,3}\right)\left(\alpha_{3,3} \alpha_{5,3}\right) .
\end{gathered}
$$




\section{Classification of KahK3 conjugacy classes for $A\left(N_{11}\right)$ :}

$\mathbf{n}=\mathbf{1}, H \cong C_{2}(|H|=2, i=1): \operatorname{rk} N_{H}=8$ and $\left(N_{H}\right)^{*} / N_{H} \cong(\mathbb{Z} / 2 \mathbb{Z})^{8}$.

$$
H_{1,1}=[\varphi]
$$

with orbits $\left\{\alpha_{1,1}, \alpha_{11,1}\right\},\left\{\alpha_{2,1}, \alpha_{10,1}\right\},\left\{\alpha_{3,1}, \alpha_{9,1}\right\},\left\{\alpha_{4,1}, \alpha_{8,1}\right\},\left\{\alpha_{5,1}, \alpha_{7,1}\right\},\left\{\alpha_{6,2}, \alpha_{7,2}\right\}$, $\left\{\alpha_{1,3}, \alpha_{6,3}\right\},\left\{\alpha_{3,3}, \alpha_{5,3}\right\}$. See [5, Ch. 16] and [17], [18 for details.

Case 9. For the Niemeier lattice

$$
N_{9}=N\left(3 D_{8}\right)=\left[3 D_{8}, \quad \varepsilon_{1,1}+\varepsilon_{2,2}+\varepsilon_{2,3}, \quad \varepsilon_{2,1}+\varepsilon_{1,2}+\varepsilon_{2,3}, \quad \varepsilon_{2,1}+\varepsilon_{2,2}+\varepsilon_{1,3}\right],
$$

the group $A\left(N_{9}\right)$ has order 6 , and it is generated by

$$
\begin{gathered}
(12)=\left(\alpha_{1,1} \alpha_{1,2}\right)\left(\alpha_{2,1} \alpha_{2,2}\right)\left(\alpha_{3,1} \alpha_{3,2}\right)\left(\alpha_{4,1} \alpha_{4,2}\right)\left(\alpha_{5,1} \alpha_{5,2}\right)\left(\alpha_{6,1} \alpha_{6,2}\right) \\
\left(\alpha_{7,1} \alpha_{7,2}\right)\left(\alpha_{8,1} \alpha_{8,2}\right), \\
(23)=\left(\alpha_{1,2} \alpha_{1,3}\right)\left(\alpha_{2,2} \alpha_{2,3}\right)\left(\alpha_{3,2} \alpha_{3,3}\right)\left(\alpha_{4,2} \alpha_{4,3}\right)\left(\alpha_{5,2} \alpha_{5,3}\right)\left(\alpha_{6,2} \alpha_{6,3}\right) \\
\left(\alpha_{7,2} \alpha_{7,3}\right)\left(\alpha_{8,2} \alpha_{8,3}\right) .
\end{gathered}
$$

Classification of KahK3 conjugacy classes for $A\left(N_{9}\right)$ :

$$
\mathbf{n}=\mathbf{1}, H \cong C_{2}(|H|=2, i=1): \operatorname{rk} N_{H}=8 \text { and }\left(N_{H}\right)^{*} / N_{H} \cong(\mathbb{Z} / 2 \mathbb{Z})^{8} \text {. }
$$

$$
H_{1,1}=[(12)]
$$

with orbits $\left\{\alpha_{1,1}, \alpha_{1,2}\right\}, \quad\left\{\alpha_{2,1}, \alpha_{2,2}\right\}, \quad\left\{\alpha_{3,1}, \alpha_{3,2}\right\}, \quad\left\{\alpha_{4,1}, \alpha_{4,2}\right\}, \quad\left\{\alpha_{5,1}, \alpha_{5,2}\right\}, \quad\left\{\alpha_{6,1}, \alpha_{6,2}\right\}$, $\left\{\alpha_{7,1} \alpha_{7,2}\right\},\left\{\alpha_{8,1} \alpha_{8,2}\right\}$. See [5, Ch. 16] and [17], [18] for details.

Case 8. For the Niemeier lattice $N_{8}=N\left(A_{15} \oplus D_{9}\right)=\left[A_{15} \oplus D_{9}, 2 \varepsilon_{1,1}+\varepsilon_{1,2}\right]$, the group $A\left(N_{8}\right)$ has order 2 , and it is generated by

$$
\begin{gathered}
\varphi=\left(\alpha_{1,1} \alpha_{15,1}\right)\left(\alpha_{2,1} \alpha_{14,1}\right)\left(\alpha_{3,1} \alpha_{13,1}\right)\left(\alpha_{4,1} \alpha_{12,1}\right)\left(\alpha_{5,1} \alpha_{11,1}\right)\left(\alpha_{6,1} \alpha_{10,1}\right) \\
\left(\alpha_{7,1} \alpha_{9,1}\right)\left(\alpha_{8,2} \alpha_{9,2}\right) .
\end{gathered}
$$

Classification of KahK3 conjugacy classes for $A\left(N_{8}\right)$ : 
$\mathbf{n}=\mathbf{1}, H \cong C_{2}(|H|=2, i=1): \operatorname{rk} N_{H}=8$ and $\left(N_{H}\right)^{*} / N_{H} \cong(\mathbb{Z} / 2 \mathbb{Z})^{8}$.

$$
H_{1,1}=[\varphi]
$$

with orbits $\left\{\alpha_{1,1}, \alpha_{15,1}\right\},\left\{\alpha_{2,1}, \alpha_{14,1}\right\},\left\{\alpha_{3,1}, \alpha_{13,1}\right\},\left\{\alpha_{4,1}, \alpha_{12,1}\right\},\left\{\alpha_{5,1}, \alpha_{11,1}\right\},\left\{\alpha_{6,1}, \alpha_{10,1}\right\}$, $\left\{\alpha_{7,1}, \alpha_{9,1}\right\},\left\{\alpha_{8,2}, \alpha_{9,2}\right\}$. See [5, Ch. 16] and [17], [18] for details.

Case 7. For the Niemeier lattice $N_{7}=N\left(D_{10} \oplus 2 E_{7}\right)=\left[D_{10} \oplus 2 E_{7}, \varepsilon_{1,1}+\varepsilon_{1,2}, \varepsilon_{3,1}+\varepsilon_{1,3}\right]$, the group $A\left(N_{7}\right)$ has order 2 , and it is generated by

$$
\begin{gathered}
\varphi=\left(\alpha_{9,1} \alpha_{10,1}\right)\left(\alpha_{1,2} \alpha_{1,3}\right)\left(\alpha_{2,2} \alpha_{2,3}\right)\left(\alpha_{3,2} \alpha_{3,3}\right)\left(\alpha_{4,2} \alpha_{4,3}\right)\left(\alpha_{5,2} \alpha_{5,3}\right) \\
\left(\alpha_{6,2} \alpha_{6,3}\right)\left(\alpha_{7,2} \alpha_{7,3}\right) .
\end{gathered}
$$

Classification of KahK3 conjugacy classes for $A\left(N_{7}\right)$ :

$\mathbf{n}=\mathbf{1}, H \cong C_{2}(|H|=2, i=1): \operatorname{rk} N_{H}=8$ and $\left(N_{H}\right)^{*} / N_{H} \cong(\mathbb{Z} / 2 \mathbb{Z})^{8}$.

$$
H_{1,1}=[\varphi]
$$

with orbits $\left\{\alpha_{9,1}, \alpha_{10,1}\right\},\left\{\alpha_{1,2}, \alpha_{1,3}\right\},\left\{\alpha_{2,2}, \alpha_{2,3}\right\}, \quad\left\{\alpha_{3,2}, \alpha_{3,3}\right\}, \quad\left\{\alpha_{4,2}, \alpha_{4,3}\right\}, \quad\left\{\alpha_{5,2}, \alpha_{5,3}\right\}$, $\left\{\alpha_{6,2}, \alpha_{6,3}\right\},\left\{\alpha_{7,2}, \alpha_{7,3}\right\}$. See [5, Ch. 16] and [17], [18] for details.

Case 6. For the Niemeier lattice $N_{6}=N\left(A_{17} \oplus E_{7}\right)=\left[A_{17} \oplus E_{7}, 3 \varepsilon_{1,1}+\varepsilon_{1,2}\right]$, the group $A\left(N_{6}\right)$ has order 2 , and it is generated by

$$
\begin{gathered}
\varphi=\left(\alpha_{1,1} \alpha_{17,1}\right)\left(\alpha_{2,1} \alpha_{16,1}\right)\left(\alpha_{3,1} \alpha_{15,1}\right)\left(\alpha_{4,1} \alpha_{14,1}\right)\left(\alpha_{5,1} \alpha_{13,1}\right)\left(\alpha_{6,1} \alpha_{12,1}\right) \\
\left(\alpha_{7,1} \alpha_{11,1}\right)\left(\alpha_{8,1} \alpha_{10,1}\right) .
\end{gathered}
$$

Classification of KahK3 conjugacy classes for $A\left(N_{6}\right)$ :

$\mathbf{n}=\mathbf{1}, H \cong C_{2}(|H|=2, i=1): \operatorname{rk} N_{H}=8$ and $\left(N_{H}\right)^{*} / N_{H} \cong(\mathbb{Z} / 2 \mathbb{Z})^{8}$.

$$
H_{1,1}=[\varphi]
$$

with orbits $\left\{\alpha_{1,1}, \alpha_{17,1}\right\},\left\{\alpha_{2,1}, \alpha_{16,1}\right\},\left\{\alpha_{3,1}, \alpha_{15,1}\right\},\left\{\alpha_{4,1}, \alpha_{14,1}\right\},\left\{\alpha_{5,1}, \alpha_{13,1}\right\},\left\{\alpha_{6,1}, \alpha_{12,1}\right\}$, $\left\{\alpha_{7,1}, \alpha_{11,1}\right\},\left\{\alpha_{8,1}, \alpha_{10,1}\right\}$. See [5, Ch. 16] and [17], [18] for details. 
Case 3. For the Niemeier lattice $N_{3}=N\left(3 E_{8}\right)=3 E_{8}$, the group $A\left(N_{3}\right)$ has order 6 , and it is generated by

$$
\begin{gathered}
(12)=\left(\alpha_{1,1} \alpha_{1,2}\right)\left(\alpha_{2,1} \alpha_{2,2}\right)\left(\alpha_{3,1} \alpha_{3,2}\right)\left(\alpha_{4,1} \alpha_{4,2}\right)\left(\alpha_{5,1} \alpha_{5,2}\right)\left(\alpha_{6,1} \alpha_{6,2}\right) \\
\left(\alpha_{7,1} \alpha_{7,2}\right)\left(\alpha_{8,1} \alpha_{8,2}\right), \\
(23)=\left(\alpha_{1,2} \alpha_{1,3}\right)\left(\alpha_{2,2} \alpha_{2,3}\right)\left(\alpha_{3,2} \alpha_{3,3}\right)\left(\alpha_{4,2} \alpha_{4,3}\right)\left(\alpha_{5,2} \alpha_{5,3}\right)\left(\alpha_{6,2} \alpha_{6,3}\right) \\
\left(\alpha_{7,2} \alpha_{7,3}\right)\left(\alpha_{8,2} \alpha_{8,3}\right) .
\end{gathered}
$$

Classification of KahK3 conjugacy classes for $A\left(N_{3}\right)$ :

$\mathbf{n}=\mathbf{1}, H \cong C_{2}(|H|=2, i=1): \operatorname{rk} N_{H}=8$ and $\left(N_{H}\right)^{*} / N_{H} \cong(\mathbb{Z} / 2 \mathbb{Z})^{8}$.

$$
H_{1,1}=[(12)]
$$

with orbits $\left\{\alpha_{1,1}, \alpha_{1,2}\right\}, \quad\left\{\alpha_{2,1}, \alpha_{2,2}\right\}, \quad\left\{\alpha_{3,1}, \alpha_{3,2}\right\}, \quad\left\{\alpha_{4,1}, \alpha_{4,2}\right\}, \quad\left\{\alpha_{5,1}, \alpha_{5,2}\right\}, \quad\left\{\alpha_{6,1}, \alpha_{6,2}\right\}$, $\left\{\alpha_{7,1} \alpha_{7,2}\right\},\left\{\alpha_{8,1} \alpha_{8,2}\right\}$. See [5, Ch. 16] and [17], [18] for details.

Cases 1, 2, 4, 5, 10. For remaining Niemeier lattices $N_{1}, N_{2}, N_{4}, N_{5}, N_{10}$, KahK3 conjugacy classes are only trivial. See [5, Ch. 16] and [17], [18] for details.

\section{Markings by Niemeier lattices of degenerations of codimension 1 of non-trivial finite symplectic auto- morphism groups of Kählerian K3 surfaces}

Like for the example of Kummer surfaces in Sect. 3, using Theorem 1 (we use Program 6 in Section 8 based on this Theorem), we classify all possible markings by Niemeier lattices $N_{j}, j=1, \ldots, 23$, of degenerations of codimension 1 of Kählerian K3 surfaces. For Niemeier lattices $N_{23}$ and $N_{22}$ we use classification of KahK3 conjugacy classes from [17] and [18. For Niemeier lattices $N_{j}, j=21, \ldots, 1$, we use similar style classification from Section 4, It will be more convenient than our classification of these cases in [17] and [18]. Here we label by $n=81, \ldots, 1$ types of abstract finite groups of symplectic automorphisms of Kählerian K3 surfaces. See [15], [11], [22] and [8].

Theorem 2. Let $N_{j}, j=1,2, \ldots, 23$, be one of Niemeier lattices.

Types of degenerations of codimension one $N_{H} \subset S \subset N_{j}$ of maximal $(\operatorname{Clos}(H)=H)$ non-trivial finite symplectic automorphism groups $H$ of Kählerian K3 surfaces which can 
be marked by the Niemeier lattice $N_{j}$ are shown in the Table $j$ below. They are given by orbits of $\alpha \in P\left(N_{j}\right)$ of Kählerian K3 conjugation classes $H$ of $A\left(N_{j}\right)$ classified in [17] for $j=23,22$ (we use notations of Cases 23 and 22 from [17] respectively), and in Section 4 for $j=21,20, \ldots, 11,9,8,7,6,3$ (we use notations of the Case $j$ from Section 4). We give the type of the root system and the isomorphism class of the discriminant group $A_{S}$ of the corresponding lattice $S=\left[N_{H}, \alpha\right]_{p r} \subset N_{j}$.

Table 23. All possible markings by $N_{23}=N\left(24 A_{1}\right)$ of degenerations of codimension one of non-trivial finite symplectic automorphism groups of Kählerian K3 surfaces:

$\mathbf{n}=\mathbf{8 1}, H \cong M_{20}(|H|=960, i=11357):$ rk $N_{H}=19$. No degenerations.

$\mathbf{n}=\mathbf{8 0}, H \cong F_{384}(|H|=384, i=18135):$ rk $N_{H}=19$. No degenerations.

$\mathbf{n}=\mathbf{7 9}, H \cong \mathfrak{A}_{6}(|H|=360, i=118):$ rk $N_{H}=19$. No degenerations.

$\mathbf{n}=\mathbf{7 8}, H \cong \mathfrak{A}_{4,4}(|H|=288, i=1026):$ rk $N_{H}=19$. No degenerations.

$\mathbf{n}=\mathbf{7 7}, H \cong T_{192}(|H|=192, i=1493):$ rk $N_{H}=19$. No degenerations.

$\mathbf{n}=\mathbf{7 6}, H \cong H_{192}(|H|=192, i=955):$ rk $N_{H}=19$. No degenerations.

$\mathbf{n}=\mathbf{7 5}, H \cong 4^{2} \mathfrak{A}_{4}(|H|=192, i=1023): \operatorname{rk} N_{H}=18,\left(N_{H}\right)^{*} / N_{H} \cong(\mathbb{Z} / 8 \mathbb{Z})^{2} \times(\mathbb{Z} / 2 \mathbb{Z})^{2}$. Degenerations: Type $16 \mathbb{A}_{1}$ with $A_{S} \cong(\mathbb{Z} / 8 \mathbb{Z}) \times(\mathbb{Z} / 2 \mathbb{Z})^{2}\left(H_{75,1}\right.$ with the orbit of $\left.\alpha_{1}\right)$.

$\mathbf{n}=\mathbf{7 4}, H \cong L_{2}(7)(|H|=168, i=42):$ rk $N_{H}=19$. No degenerations.

$\mathbf{n}=\mathbf{7 0}, H \cong \mathfrak{S}_{5}(|H|=120, i=34):$ rk $N_{H}=19$. No degenerations.

$\mathbf{n}=65, H \cong 2^{4} D_{6}(|H|=96, i=227): \operatorname{rk} N_{H}=18,\left(N_{H}\right)^{*} / N_{H} \cong \mathbb{Z} / 24 \mathbb{Z} \times \mathbb{Z} / 4 \mathbb{Z} \times$ $(\mathbb{Z} / 2 \mathbb{Z})^{2}$. Degenerations: $4 \mathbb{A}_{1}$ with $A_{S} \cong \mathbb{Z} / 12 \mathbb{Z} \times(\mathbb{Z} / 4 \mathbb{Z})^{2}\left(H_{65,3}\right.$ with the orbit of $\alpha_{3}$ or $\alpha_{10} ; H_{65,4}$ with the orbit of $\alpha_{3}, \alpha_{4}$ or $\left.\alpha_{7}\right) ; 8 \mathbb{A}_{1}$ with $A_{S} \cong \mathbb{Z} / 24 \mathbb{Z} \times(\mathbb{Z} / 2 \mathbb{Z})^{2}\left(H_{65,2}\right.$ with the obit of $\alpha_{1} ; H_{65,4}$ with the orbit of $\left.\alpha_{1}\right) ; 12 \mathbb{A}_{1}$ with $A_{S} \cong(\mathbb{Z} / 4 \mathbb{Z})^{3}\left(H_{65,3}\right.$ with the orbit of $\left.\alpha_{1}\right)$; $16 \mathbb{A}_{1}$ with $A_{S} \cong \mathbb{Z} / 12 \mathbb{Z} \times(\mathbb{Z} / 2 \mathbb{Z})^{2}\left(H_{65,1}\right.$ with the orbit of $\left.\alpha_{1}\right)$.

$\mathbf{n}=63, H \cong M_{9}(|H|=72, i=41):$ rk $N_{H}=19$. No degenerations.

$\mathbf{n}=62, H \cong N_{72}(|H|=72, i=40):$ rk $N_{H}=19$. No degenerations.

$\mathbf{n}=\mathbf{6 1}, H \cong \mathfrak{A}_{4,3}(|H|=72, i=43):$ rk $N_{H}=18$ and $\left(N_{H}\right)^{*} / N_{H} \cong(\mathbb{Z} / 12 \mathbb{Z})^{2} \times \mathbb{Z} / 3 \mathbb{Z}$. Degenerations: $3 \mathbb{A}_{1}$ with $A_{S} \cong(\mathbb{Z} / 12 \mathbb{Z})^{2} \times \mathbb{Z} / 2 \mathbb{Z}\left(H_{61,1}\right.$ with the orbit of $\alpha_{7}$ or $\left.\alpha_{16}\right) ; 12 \mathbb{A}_{1}$ with $A_{S} \cong \mathbb{Z} / 24 \mathbb{Z} \times \mathbb{Z} / 3 \mathbb{Z}\left(H_{61,1}\right.$ with the orbit of $\left.\alpha_{2}\right)$. 
$\mathbf{n}=\mathbf{5 6}, H \cong \Gamma_{25} a_{1}(|H|=64, i=138): \operatorname{rk} N_{H}=18,\left(N_{H}\right)^{*} / N_{H} \cong \mathbb{Z} / 8 \mathbb{Z} \times(\mathbb{Z} / 4 \mathbb{Z})^{3}$. Degenerations: $8 \mathbb{A}_{1}$ with $A_{S} \cong \mathbb{Z} / 8 \mathbb{Z} \times(\mathbb{Z} / 4 \mathbb{Z})^{2}\left(H_{56,1}\right.$ with the orbit of $\alpha_{1}$ or $\left.\alpha_{6}\right) ; 16 \mathbb{A}_{1}$ with $A_{S} \cong(\mathbb{Z} / 4 \mathbb{Z})^{3}\left(H_{56,2}\right.$ with the orbit of $\left.\alpha_{2}\right)$.

$\mathbf{n}=55, H \cong \mathfrak{A}_{5}(|H|=60, i=5): \operatorname{rk} N_{H}=18$ and $\left(N_{H}\right)^{*} / N_{H} \cong \mathbb{Z} / 30 \mathbb{Z} \times \mathbb{Z} / 10 \mathbb{Z}$. Degenerations: $\mathbb{A}_{1}$ with $A_{S} \cong \mathbb{Z} / 30 \mathbb{Z} \times \mathbb{Z} / 10 \mathbb{Z} \times \mathbb{Z} / 2 \mathbb{Z}\left(H_{55,1}\right.$ with the orbit of $\alpha_{1}, \alpha_{10}$ or $\alpha_{24}$; $H_{55,2}$ with the orbit of $\alpha_{1}, \alpha_{5}, \alpha_{21}$ or $\left.\alpha_{24}\right) ; 5 \mathbb{A}_{1}$ with $A_{S} \cong \mathbb{Z} / 30 \mathbb{Z} \times(\mathbb{Z} / 2 \mathbb{Z})^{2}\left(H_{55,1}\right.$ with the orbit of $\alpha_{2} ; H_{55,2}$ with the orbit of $\left.\alpha_{4}\right) ; 6 \mathbb{A}_{1}$ with $A_{S} \cong \mathbb{Z} / 20 \mathbb{Z} \times \mathbb{Z} / 5 \mathbb{Z}\left(H_{55,1}\right.$ with the orbit of $\left.\alpha_{4}\right) ; 10 \mathbb{A}_{1}$ with $A_{S} \cong \mathbb{Z} / 60 \mathbb{Z}\left(H_{55,1}\right.$ with the orbit of $\left.\alpha_{3}\right) ; 15 \mathbb{A}_{1}$ with $A_{S} \cong \mathbb{Z} / 10 \mathbb{Z} \times(\mathbb{Z} / 2 \mathbb{Z})^{2}$ $\left(H_{55,2}\right.$ with the orbit of $\left.\alpha_{2}\right)$.

$\mathbf{n}=\mathbf{5 4}, H \cong T_{48}(|H|=48, i=29):$ rk $N_{H}=19$. No degenerations.

$\mathbf{n}=\mathbf{5 1}, H \cong C_{2} \times \mathfrak{S}_{4}(|H|=48, i=48): \operatorname{rk} N_{H}=18,\left(N_{H}\right)^{*} / N_{H} \cong(\mathbb{Z} / 12 \mathbb{Z})^{2} \times(\mathbb{Z} / 2 \mathbb{Z})^{2}$. Degenerations: $2 \mathbb{A}_{1}$ with $A_{S} \cong(\mathbb{Z} / 12 \mathbb{Z})^{2} \times \mathbb{Z} / 4 \mathbb{Z}\left(H_{51,2}\right.$ with the orbit of $\alpha_{2}$ or $\alpha_{10} ; H_{51,3}$ with the orbit of $\alpha_{2} ; H_{51,4}$ with the orbit of $\alpha_{3}$ or $\left.\alpha_{6}\right) ; 4 \mathbb{A}_{1}$ with $A_{S} \cong \mathbb{Z} / 24 \mathbb{Z} \times \mathbb{Z} / 6 \mathbb{Z} \times \mathbb{Z} / 2 \mathbb{Z}\left(H_{51,2}\right.$ with the orbit of $\alpha_{1} ; H_{51,4}$ with the orbit of $\alpha_{1}$ or $\left.\alpha_{5}\right) ; 6 \mathbb{A}_{1}$ with $A_{S} \cong \mathbb{Z} / 12 \mathbb{Z} \times(\mathbb{Z} / 4 \mathbb{Z})^{2}\left(H_{51,3}\right.$ with the orbit of $\alpha_{4} ; H_{51,4}$ with the orbit of $\alpha_{2}$ or $\left.\alpha_{7}\right) ; 8 \mathbb{A}_{1}$ with $A_{S} \cong \mathbb{Z} / 12 \mathbb{Z} \times \mathbb{Z} / 6 \mathbb{Z} \times \mathbb{Z} / 2 \mathbb{Z}$ $\left(H_{51,1}\right.$ with the orbit of $\alpha_{1}$ or $\alpha_{4} ; H_{51,3}$ with the orbit of $\left.\alpha_{1}\right) ; 12 \mathbb{A}_{1}$ with $A_{S} \cong \mathbb{Z} / 24 \mathbb{Z} \times(\mathbb{Z} / 2 \mathbb{Z})^{2}$ $\left(H_{51,2}\right.$ with the orbit of $\left.\alpha_{4}\right)$.

$\mathbf{n}=49, H \cong 2 C_{3}(|H|=48, i=50): \operatorname{rk} N_{H}=17,\left(N_{H}\right)^{*} / N_{H} \cong \mathbb{Z} / 24 \mathbb{Z} \times(\mathbb{Z} / 2 \mathbb{Z})^{4}$. Degenerations of codimension 1 : $4 \mathbb{A}_{1}$ with $A_{S} \cong \mathbb{Z} / 12 \mathbb{Z} \times \mathbb{Z} / 4 \mathbb{Z} \times(\mathbb{Z} / 2 \mathbb{Z})^{2}\left(H_{49,1}\right.$ with the orbit of $\alpha_{2}$ or $\alpha_{4} ; H_{49,3}$ with the orbit of $\alpha_{2}, \alpha_{4}, \alpha_{8}, \alpha_{10}$ or $\left.\alpha_{11}\right) ; 12 \mathbb{A}_{1}$ with $A_{S} \cong$ $(\mathbb{Z} / 4 \mathbb{Z})^{2} \times(\mathbb{Z} / 2 \mathbb{Z})^{2}\left(H_{49,1}\right.$ with the orbit of $\left.\alpha_{1}\right) ; 16 \mathbb{A}_{1}$ with $A_{S} \cong \mathbb{Z} / 6 \mathbb{Z} \times(\mathbb{Z} / 2 \mathbb{Z})^{3}\left(H_{49,2}\right.$ with the orbit of $\left.\alpha_{2}\right)$.

$\mathbf{n}=\mathbf{4 8}, H \cong \mathfrak{S}_{3,3}(|H|=36, i=10): \operatorname{rk} N_{H}=18,\left(N_{H}\right)^{*} / N_{H} \cong \mathbb{Z} / 18 \mathbb{Z} \times \mathbb{Z} / 6 \mathbb{Z} \times(\mathbb{Z} / 3 \mathbb{Z})^{2}$. Degenerations: $3 \mathbb{A}_{1}$ with $A_{S} \cong \mathbb{Z} / 18 \mathbb{Z} \times(\mathbb{Z} / 6 \mathbb{Z})^{2}\left(H_{48,1}\right.$ with the orbit of $\alpha_{5}$ or $\left.\alpha_{7}\right) ; 6 \mathbb{A}_{1}$ with $A_{S} \cong \mathbb{Z} / 36 \mathbb{Z} \times(\mathbb{Z} / 3 \mathbb{Z})^{2}\left(H_{48,1}\right.$ with the orbit of $\left.\alpha_{2}\right) ; 9 \mathbb{A}_{1}$ with $A_{S} \cong(\mathbb{Z} / 6 \mathbb{Z})^{3}\left(H_{48,1}\right.$ with the orbit of $\alpha_{4}$ ).

$\mathbf{n}=46, H \cong 3^{2} C_{4}(|H|=36, i=9): \operatorname{rk} N_{H}=18$ and $\left(N_{H}\right)^{*} / N_{H} \cong \mathbb{Z} / 18 \mathbb{Z} \times \mathbb{Z} / 6 \mathbb{Z} \times \mathbb{Z} / 3 \mathbb{Z}$. Degenerations: $6 \mathbb{A}_{1}$ with $A_{S} \cong \mathbb{Z} / 36 \mathbb{Z} \times \mathbb{Z} / 3 \mathbb{Z}\left(H_{46,1}\right.$ with the orbit of $\alpha_{1}$ or $\left.\alpha_{3}\right) ; 9 \mathbb{A}_{1}$ with $A_{S} \cong(\mathbb{Z} / 6 \mathbb{Z})^{2} \times \mathbb{Z} / 2 \mathbb{Z}\left(H_{46,1}\right.$ with the orbit of $\left.\alpha_{2}\right) ;$

$\mathbf{n}=40, H \cong Q_{8} * Q_{8}(|H|=32, i=49): \operatorname{rk} N_{H}=17,\left(N_{H}\right)^{*} / N_{H} \cong(\mathbb{Z} / 4 \mathbb{Z})^{5}$. Degenerations of codimension 1 : $8 \mathbb{A}_{1}$ with $A_{S} \cong(\mathbb{Z} / 4 \mathbb{Z})^{4}\left(H_{40,1}\right.$ with the orbit of $\alpha_{1}$ or $\left.\alpha_{3}\right)$.

$\mathbf{n}=39, H \cong 24 C_{2}(|H|=32, i=27): \operatorname{rk} N_{H}=17,\left(N_{H}\right)^{*} / N_{H} \cong \mathbb{Z} / 8 \mathbb{Z} \times(\mathbb{Z} / 4 \mathbb{Z})^{2} \times$ $(\mathbb{Z} / 2 \mathbb{Z})^{2}$. Degenerations of codimension $1: 4 \mathbb{A}_{1}$ with $A_{S} \cong(\mathbb{Z} / 4 \mathbb{Z})^{4}\left(H_{39,2}\right.$ with the orbit of $\alpha_{2}, \alpha_{3}$ or $\left.\alpha_{4}\right) ; 8 \mathbb{A}_{1}$ with $A_{S} \cong \mathbb{Z} / 8 \mathbb{Z} \times \mathbb{Z} / 4 \mathbb{Z} \times(\mathbb{Z} / 2 \mathbb{Z})^{2}\left(H_{39,2}\right.$ with the orbit of $\alpha_{5} ; H_{39,3}$ with the orbit of $\alpha_{3}$ or $\left.\alpha_{5}\right) ; 16 \mathbb{A}_{1}$ with $A_{S} \cong(\mathbb{Z} / 4 \mathbb{Z})^{2} \times(\mathbb{Z} / 2 \mathbb{Z})^{2}\left(H_{39,1}\right.$ with the orbit of $\left.\alpha_{2}\right)$. 
$\mathbf{n}=\mathbf{3 4}, H \cong \mathfrak{S}_{4}(|H|=24, i=12): \operatorname{rk} N_{H}=17$ and $\left(N_{H}\right)^{*} / N_{H} \cong(\mathbb{Z} / 12 \mathbb{Z})^{2} \times \mathbb{Z} / 4 \mathbb{Z}$. Degenerations of codimension 1 : $\mathbb{A}_{1}$ with $A_{S} \cong(\mathbb{Z} / 12 \mathbb{Z})^{2} \times \mathbb{Z} / 4 \mathbb{Z} \times \mathbb{Z} / 2 \mathbb{Z}\left(H_{34,1}\right.$ with the orbit of $\alpha_{5}, \alpha_{6}, \alpha_{17}$ or $\alpha_{21} ; H_{34,2}$ with the orbit of $\alpha_{6}, \alpha_{21}$ or $\alpha_{24} ; H_{34,3}$ with the orbit of $\alpha_{5}$ or $\alpha_{21} ; H_{34,4}$ with the orbit of $\alpha_{10}$ or $\left.\alpha_{24}\right) ; 2 \mathbb{A}_{1}$ with $A_{S} \cong(\mathbb{Z} / 12 \mathbb{Z})^{2} \times(\mathbb{Z} / 2 \mathbb{Z})^{2}\left(H_{34,2}\right.$ with the orbit of $\alpha_{4} ; H_{34,3}$ with the orbit of $\left.\alpha_{4}\right) ; 3 \mathbb{A}_{1}$ with $A_{S} \cong \mathbb{Z} / 12 \mathbb{Z} \times(\mathbb{Z} / 4 \mathbb{Z})^{2} \times \mathbb{Z} / 2 \mathbb{Z}\left(H_{34,2}\right.$ with the orbit of $\alpha_{2} ; H_{34,4}$ with the orbit of $\alpha_{2}$ or $\left.\alpha_{3}\right) ; 4 \mathbb{A}_{1}$ with $A_{S} \cong \mathbb{Z} / 24 \mathbb{Z} \times \mathbb{Z} / 12 \mathbb{Z}\left(H_{34,2}\right.$ with the orbit of $\alpha_{1} ; H_{34,3}$ with the orbit of $\alpha_{1}$ or $\alpha_{10} ; H_{34,4}$ with the orbit of $\alpha_{1}$ or $\left.\alpha_{5}\right) ; 6 \mathbb{A}_{1}$ with $A_{S} \cong \mathbb{Z} / 12 \mathbb{Z} \times \mathbb{Z} / 4 \mathbb{Z} \times(\mathbb{Z} / 2 \mathbb{Z})^{2}\left(H_{34,1}\right.$ with the orbit of $\alpha_{1}$ or $\alpha_{8} ; H_{34,3}$ with the orbit of $\alpha_{2}$ or $\left.\alpha_{3}\right) ; 8 \mathbb{A}_{1}$ with $A_{S} \cong(\mathbb{Z} / 12 \mathbb{Z})^{2}\left(H_{34,1}\right.$ with the orbit of $\alpha_{2} ; H_{34,4}$ with the orbit of $\left.\alpha_{4}\right) ; 12 \mathbb{A}_{1}$ with $A_{S} \cong \mathbb{Z} / 24 \mathbb{Z} \times \mathbb{Z} / 4 \mathbb{Z}\left(H_{34,2}\right.$ with the orbit of $\left.\alpha_{3}\right)$.

$\mathbf{n}=33, H \cong C_{7} \rtimes C_{3}(|H|=21, i=1): \operatorname{rk} N_{H}=18$ and $\left(N_{H}\right)^{*} / N_{H} \cong(\mathbb{Z} / 7 \mathbb{Z})^{3}$. Degenerations: $7 \mathbb{A}_{1}$ with $A_{S} \cong \mathbb{Z} / 14 \mathbb{Z} \times \mathbb{Z} / 7 \mathbb{Z}\left(H_{33,1}\right.$ with the orbit of $\alpha_{1}, \alpha_{3}$ or $\left.\alpha_{11}\right)$.

$\mathbf{n}=\mathbf{3 2}, H \cong \operatorname{Hol}\left(C_{5}\right)(|H|=20, i=3): \operatorname{rk} N_{H}=18$ and $\left(N_{H}\right)^{*} / N_{H} \cong(\mathbb{Z} / 10 \mathbb{Z})^{2} \times \mathbb{Z} / 5 \mathbb{Z}$. Degenerations: $2 \mathbb{A}_{1}$ with $A_{S} \cong \mathbb{Z} / 20 \mathbb{Z} \times(\mathbb{Z} / 5 \mathbb{Z})^{2}\left(H_{32,1}\right.$ with the orbit of $\left.\alpha_{19}\right) ; 5 \mathbb{A}_{1}$ with $A_{S} \cong(\mathbb{Z} / 10 \mathbb{Z})^{2} \times \mathbb{Z} / 2 \mathbb{Z}\left(H_{32,1}\right.$ with the orbit of $\alpha_{3}$ or $\left.\alpha_{4}\right) ; 10 \mathbb{A}_{1}$ with $A_{S} \cong \mathbb{Z} / 20 \mathbb{Z} \times \mathbb{Z} / 5 \mathbb{Z}$ $\left(H_{32,1}\right.$ with the orbit of $\left.\alpha_{2}\right)$.

$\mathbf{n}=\mathbf{3 0}, H \cong \mathfrak{A}_{3,3}(|H|=18, i=4): \operatorname{rk} N_{H}=16$ and $\left(N_{H}\right)^{*} / N_{H} \cong \mathbb{Z} / 9 \mathbb{Z} \times(\mathbb{Z} / 3 \mathbb{Z})^{4}$. Degenerations of codimension 1 : $3 \mathbb{A}_{1}$ with $A_{S} \cong \mathbb{Z} / 18 \mathbb{Z} \times(\mathbb{Z} / 3 \mathbb{Z})^{3}\left(H_{30,1}\right.$ with the orbit of $\alpha_{4}, \alpha_{5}, \alpha_{11}$ or $\left.\alpha_{15}\right) ; 9 \mathbb{A}_{1}$ with $A_{S} \cong \mathbb{Z} / 6 \mathbb{Z} \times(\mathbb{Z} / 3 \mathbb{Z})^{3}\left(H_{30,1}\right.$ with the orbit of $\left.\alpha_{1}\right)$.

$\mathbf{n}=\mathbf{2 6}, H \cong S D_{16}(|H|=16, i=8): \operatorname{rk} N_{H}=18$ and $\left(N_{H}\right)^{*} / N_{H} \cong(\mathbb{Z} / 8 \mathbb{Z})^{2} \times \mathbb{Z} / 4 \mathbb{Z} \times$ $\mathbb{Z} / 2 \mathbb{Z}$. Degenerations: $8 \mathbb{A}_{1}$ with $A_{S} \cong \mathbb{Z} / 16 \mathbb{Z} \times \mathbb{Z} / 4 \mathbb{Z} \times \mathbb{Z} / 2 \mathbb{Z}\left(H_{26,1}\right.$ with the orbit of $\alpha_{1}$ or $\left.\alpha_{3}\right)$.

$\mathbf{n}=\mathbf{2 2}, H \cong C_{2} \times D_{8}(|H|=16, i=11): \operatorname{rk} N_{H}=16,\left(N_{H}\right)^{*} / N_{H} \cong(\mathbb{Z} / 4 \mathbb{Z})^{4} \times(\mathbb{Z} / 2 \mathbb{Z})^{2}$. Degenerations of codimension 1: $2 \mathbb{A}_{1}$ with $A_{S} \cong(\mathbb{Z} / 4 \mathbb{Z})^{5}\left(H_{22,2}\right.$ with the orbit of $\alpha_{12}$ or $\alpha_{16} ; H_{22,3}$ with the orbit of $\alpha_{8}, \alpha_{11}, \alpha_{12}$ or $\left.\alpha_{16}\right) ; 4 \mathbb{A}_{1}$ with $A_{S} \cong \mathbb{Z} / 8 \mathbb{Z} \times(\mathbb{Z} / 4 \mathbb{Z})^{2} \times(\mathbb{Z} / 2 \mathbb{Z})^{2}$ $\left(H_{22,2}\right.$ with the orbit of $\alpha_{6}$ or $\alpha_{8} ; H_{22,3}$ with the orbit of $\alpha_{1}, \alpha_{2}, \alpha_{4}$ or $\left.\alpha_{6}\right)$. $8 \mathbb{A}_{1}$ with $A_{S} \cong(\mathbb{Z} / 4 \mathbb{Z})^{3} \times(\mathbb{Z} / 2 \mathbb{Z})^{2}\left(H_{22,1}\right.$ with the orbit of $\alpha_{2}$ or $\alpha_{4} ; H_{22,2}$ with the orbit of $\left.\alpha_{2}\right)$.

$\mathbf{n}=\mathbf{2 1}, H \cong C_{2}^{4}(|H|=16, i=14): \operatorname{rk} N_{H}=15,\left(N_{H}\right)^{*} / N_{H} \cong \mathbb{Z} / 8 \mathbb{Z} \times(\mathbb{Z} / 2 \mathbb{Z})^{6}$. Degenerations of codimension $1: 4 \mathbb{A}_{1}$ with $A_{S} \cong(\mathbb{Z} / 4 \mathbb{Z})^{2} \times(\mathbb{Z} / 2 \mathbb{Z})^{4}\left(H_{21,1}\right.$ with the orbit of $\alpha_{1}, \alpha_{2}, \alpha_{3}, \alpha_{8}$ or $\left.\alpha_{12}\right) ; 16 \mathbb{A}_{1}$ with $A_{S} \cong(\mathbb{Z} / 2 \mathbb{Z})^{6}\left(H_{21,2}\right.$ with the orbit of $\left.\alpha_{1}\right)$.

$\mathbf{n}=\mathbf{1 8}, H \cong D_{12}(|H|=12, i=4): \operatorname{rk} N_{H}=16$ and $\left(N_{H}\right)^{*} / N_{H} \cong(\mathbb{Z} / 6 \mathbb{Z})^{4}$. Degenerations of codimension 1 : $\mathbb{A}_{1}$ with $A_{S} \cong(\mathbb{Z} / 6 \mathbb{Z})^{4} \times \mathbb{Z} / 2 \mathbb{Z}\left(H_{18,1}\right.$ with the orbit of $\alpha_{1}$ or $\left.\alpha_{6}\right)$; $2 \mathbb{A}_{1}$ with $A_{S} \cong \mathbb{Z} / 12 \mathbb{Z} \times(\mathbb{Z} / 6 \mathbb{Z})^{2} \times \mathbb{Z} / 3 \mathbb{Z}\left(H_{18,1}\right.$ with the orbit of $\alpha_{15}$ or $\left.\alpha_{16}\right) ; 3 \mathbb{A}_{1}$ with $A_{S} \cong(\mathbb{Z} / 6 \mathbb{Z})^{3} \times(\mathbb{Z} / 2 \mathbb{Z})^{2}\left(H_{18,1}\right.$ with the orbit of $\alpha_{4}$ or $\left.\alpha_{5}\right) ; 6 \mathbb{A}_{1}$ with $A_{S} \cong \mathbb{Z} / 12 \mathbb{Z} \times(\mathbb{Z} / 6 \mathbb{Z})^{2}$ $\left(H_{18,1}\right.$ with the orbit of $\alpha_{2}$ or $\left.\alpha_{8}\right)$. 
$\mathbf{n}=\mathbf{1 7}, H \cong \mathfrak{A}_{4}(|H|=12, i=3): \operatorname{rk} N_{H}=16$ and $\left(N_{H}\right)^{*} / N_{H} \cong(\mathbb{Z} / 12 \mathbb{Z})^{2} \times(\mathbb{Z} / 2 \mathbb{Z})^{2}$. Degenerations of codimension 1 : $\mathbb{A}_{1}$ with $A_{S} \cong(\mathbb{Z} / 12 \mathbb{Z})^{2} \times(\mathbb{Z} / 2 \mathbb{Z})^{3}\left(H_{17,1}\right.$ with the orbit of $\alpha_{5}, \alpha_{15}, \alpha_{17}, \alpha_{21}$ or $\alpha_{24} ; H_{17,2}$ with the orbit of $\alpha_{5}, \alpha_{6}, \alpha_{10}$ or $\alpha_{17} ; H_{17,3}$ with the orbit of $\alpha_{10}$ or $\left.\alpha_{15}\right) ; 3 \mathbb{A}_{1}$ with $A_{S} \cong \mathbb{Z} / 12 \mathbb{Z} \times \mathbb{Z} / 4 \mathbb{Z} \times(\mathbb{Z} / 2 \mathbb{Z})^{3}\left(H_{17,1}\right.$ with the orbit of $\alpha_{1} ; H_{17,3}$ with the orbit of $\alpha_{1}$ or $\left.\alpha_{6}\right) ; 4 \mathbb{A}_{1}$ with $A_{S} \cong \mathbb{Z} / 24 \mathbb{Z} \times \mathbb{Z} / 6 \mathbb{Z} \times \mathbb{Z} / 2 \mathbb{Z}\left(H_{17,1}\right.$ with the orbit of $\alpha_{2} ; H_{17,2}$ with the orbit of $\alpha_{2}$ or $\alpha_{4} ; H_{17,3}$ with the orbit of $\alpha_{2}, \alpha_{3}, \alpha_{8}$ or $\left.\alpha_{11}\right) ; 6 \mathbb{A}_{1}$ with $A_{S} \cong \mathbb{Z} / 12 \mathbb{Z} \times(\mathbb{Z} / 4 \mathbb{Z})^{2}\left(H_{17,2}\right.$ with the orbit of $\alpha_{2}$ or $\left.\alpha_{4}\right) ; 12 \mathbb{A}_{1}$ with $A_{S} \cong \mathbb{Z} / 24 \mathbb{Z} \times(\mathbb{Z} / 2 \mathbb{Z})^{2}$ $\left(H_{17,1}\right.$ with the orbit of $\left.\alpha_{3}\right)$.

$\mathbf{n}=\mathbf{1 6}, H \cong D_{10}(|H|=10, i=1): \operatorname{rk} N_{H}=16$ and $\left(N_{H}\right)^{*} / N_{H} \cong(\mathbb{Z} / 5 \mathbb{Z})^{4}$. Degenerations of codimension 1 : $\mathbb{A}_{1}$ with $A_{S} \cong \mathbb{Z} / 10 \mathbb{Z} \times(\mathbb{Z} / 5 \mathbb{Z})^{3}\left(H_{16,1}\right.$ with the orbit of $\alpha_{1}, \alpha_{6}, \alpha_{19}$ or $\left.\alpha_{22}\right) ; 5 \mathbb{A}_{1}$ with $A_{S} \cong \mathbb{Z} / 10 \mathbb{Z} \times(\mathbb{Z} / 5 \mathbb{Z})^{2}\left(H_{16,1}\right.$ with the orbit of $\alpha_{2}, \alpha_{3}, \alpha_{4}$ or $\left.\alpha_{5}\right)$.

$\mathbf{n}=\mathbf{1 2}, H \cong Q_{8}(|H|=8, i=4): \operatorname{rk} N_{H}=17,\left(N_{H}\right)^{*} / N_{H} \cong(\mathbb{Z} / 8 \mathbb{Z})^{2} \times(\mathbb{Z} / 2 \mathbb{Z})^{3}$. Degenerations of codimension 1 : $8 \mathbb{A}_{1}$ with $A_{S} \cong \mathbb{Z} / 16 \mathbb{Z} \times(\mathbb{Z} / 2 \mathbb{Z})^{3}\left(H_{12,1}\right.$ with the orbit of $\alpha_{1}$ or $\left.\alpha_{4}\right)$.

$\mathbf{n}=\mathbf{1 0}, H \cong D_{8}(|H|=8, i=3): \operatorname{rk} N_{H}=15$ and $\left(N_{H}\right)^{*} / N_{H} \cong(\mathbb{Z} / 4 \mathbb{Z})^{5}$. Degenerations of codimension $1: \mathbb{A}_{1}$ with $A_{S} \cong(\mathbb{Z} / 4 \mathbb{Z})^{5} \times \mathbb{Z} / 2 \mathbb{Z}\left(H_{10,1}\right.$ with the orbit of $\alpha_{1}, \alpha_{5}, \alpha_{6}$ or $\alpha_{24}$; $H_{10,2}$ with the orbit of $\alpha_{1}$ or $\left.\alpha_{6}\right) ; 2 \mathbb{A}_{1}$ with $A_{S} \cong(\mathbb{Z} / 4 \mathbb{Z})^{4} \times(\mathbb{Z} / 2 \mathbb{Z})^{2}\left(H_{10,1}\right.$ with the orbit of $\alpha_{10}$ or $\alpha_{15} ; H_{10,2}$ with the orbit of $\alpha_{2}, \alpha_{10}$ or $\left.\alpha_{16}\right) ; 4 \mathbb{A}_{1}$ with $A_{S} \cong \mathbb{Z} / 8 \mathbb{Z} \times(\mathbb{Z} / 4 \mathbb{Z})^{3}\left(H_{10,1}\right.$ with the orbit of $\alpha_{3}$ or $\alpha_{7} ; H_{10,2}$ with the orbit of $\alpha_{3}, \alpha_{4}, \alpha_{5}$ or $\left.\alpha_{7}\right) ; 8 \mathbb{A}_{1}$ with $A_{S} \cong(\mathbb{Z} / 4 \mathbb{Z})^{4}$ $\left(H_{10,1}\right.$ with the orbit of $\left.\alpha_{2}\right)$.

$\mathbf{n}=\mathbf{9}, H \cong C_{2}^{3}(|H|=8, i=5): \operatorname{rk} N_{H}=14,\left(N_{H}\right)^{*} / N_{H} \cong(\mathbb{Z} / 4 \mathbb{Z})^{2} \times(\mathbb{Z} / 2 \mathbb{Z})^{6}$. Degenerations of codimension 1 : $2 \mathbb{A}_{1}$ with $A_{S} \cong(\mathbb{Z} / 4 \mathbb{Z})^{3} \times(\mathbb{Z} / 2 \mathbb{Z})^{4}\left(H_{9,1}\right.$ with the orbit of $\alpha_{5}$ or $\alpha_{6} ; H_{9,3}$ with the orbit of $\alpha_{6}, \alpha_{8}, \alpha_{10}, \alpha_{11}, \alpha_{12}, \alpha_{15}$ or $\left.\alpha_{16}\right) ; 4 \mathbb{A}_{1}$ with $A_{S} \cong \mathbb{Z} / 8 \mathbb{Z} \times(\mathbb{Z} / 2 \mathbb{Z})^{6}$ $\left(H_{9,1}\right.$ with the orbit of $\alpha_{2}, \alpha_{3}, \alpha_{4}$ or $\alpha_{7} ; H_{9,4}$ with the orbit of $\alpha_{2}$ or $\left.\alpha_{4}\right) ; 8 \mathbb{A}_{1}$ with $A_{S} \cong$ $\mathbb{Z} / 4 \mathbb{Z} \times(\mathbb{Z} / 2 \mathbb{Z})^{6}\left(H_{9,2}\right.$ with the orbit of $\alpha_{2}$ or $\alpha_{4} ; H_{9,3}$ with the orbit of $\left.\alpha_{2}\right)$.

$\mathbf{n}=6, H \cong D_{6}(|H|=6, i=1)$ : $\operatorname{rk} N_{H}=14$ and $\left(N_{H}\right)^{*} / N_{H} \cong(\mathbb{Z} / 6 \mathbb{Z})^{2} \times(\mathbb{Z} / 3 \mathbb{Z})^{3}$. Degenerations of codimension 1 : $\mathbb{A}_{1}$ with $A_{S} \cong(\mathbb{Z} / 6 \mathbb{Z})^{3} \times(\mathbb{Z} / 3 \mathbb{Z})^{2}\left(H_{6,1}\right.$ with the orbit of $\alpha_{1}, \alpha_{5}, \alpha_{6}$ or $\left.\alpha_{24}\right) ; 2 \mathbb{A}_{1}$ with $A_{S} \cong \mathbb{Z} / 12 \mathbb{Z} \times(\mathbb{Z} / 3 \mathbb{Z})^{4}\left(H_{6,1}\right.$ with the orbit of $\left.\alpha_{16}\right) ; 3 \mathbb{A}_{1}$ with $A_{S} \cong(\mathbb{Z} / 6 \mathbb{Z})^{3} \times \mathbb{Z} / 3 \mathbb{Z}\left(H_{6,1}\right.$ with the orbit of $\alpha_{3}, \alpha_{7}, \alpha_{10}$ or $\left.\alpha_{11}\right) ; 6 \mathbb{A}_{1}$ with $A_{S} \cong$ $\mathbb{Z} / 12 \mathbb{Z} \times(\mathbb{Z} / 3 \mathbb{Z})^{3}\left(H_{6,1}\right.$ with the orbit of $\left.\alpha_{2}\right)$.

$\mathbf{n}=4, H \cong C_{4}(|H|=4, i=1): \operatorname{rk} N_{H}=14$ and $\left(N_{H}\right)^{*} / N_{H} \cong(\mathbb{Z} / 4 \mathbb{Z})^{4} \times(\mathbb{Z} / 2 \mathbb{Z})^{2}$. Degenerations of codimension 1 : $\mathbb{A}_{1}$ with $A_{S} \cong(\mathbb{Z} / 4 \mathbb{Z})^{4} \times(\mathbb{Z} / 2 \mathbb{Z})^{3}\left(H_{4,1}\right.$ with the orbit of $\alpha_{1}, \alpha_{4}, \alpha_{17}$ or $\left.\alpha_{18}\right) ; 2 \mathbb{A}_{1}$ with $A_{S} \cong(\mathbb{Z} / 4 \mathbb{Z})^{5}\left(H_{4,1}\right.$ with the orbit of $\alpha_{8}$ or $\left.\alpha_{12}\right) ; 4 \mathbb{A}_{1}$ with $A_{S} \cong \mathbb{Z} / 8 \mathbb{Z} \times(\mathbb{Z} / 4 \mathbb{Z})^{2} \times(\mathbb{Z} / 2 \mathbb{Z})^{2}\left(H_{4,1}\right.$ with the orbit of $\alpha_{2}, \alpha_{3}, \alpha_{7}$ or $\left.\alpha_{11}\right) ;$

$\mathbf{n}=\mathbf{3}, H \cong C_{2}^{2}(|H|=4, i=2): \operatorname{rk} N_{H}=12$ and $\left(N_{H}\right)^{*} / N_{H} \cong(\mathbb{Z} / 4 \mathbb{Z})^{2} \times(\mathbb{Z} / 2 \mathbb{Z})^{6}$. Degenerations of codimension 1 : $\mathbb{A}_{1}$ with $A_{S} \cong(\mathbb{Z} / 4 \mathbb{Z})^{2} \times(\mathbb{Z} / 2 \mathbb{Z})^{7}\left(H_{3,1}\right.$ with the orbit of 
$\alpha_{1}, \alpha_{5}, \alpha_{6}, \alpha_{10}, \alpha_{15}, \alpha_{17}, \alpha_{21}$ or $\alpha_{24} ; H_{3,2}$ with the orbit of $\alpha_{1}, \alpha_{5}, \alpha_{6}$ or $\left.\alpha_{21}\right) ; 2 \mathbb{A}_{1}$ with $A_{S} \cong(\mathbb{Z} / 4 \mathbb{Z})^{3} \times(\mathbb{Z} / 2 \mathbb{Z})^{4}\left(H_{3,2}\right.$ with the orbit of $\alpha_{8}, \alpha_{10}, \alpha_{11}, \alpha_{12}, \alpha_{15}$ or $\alpha_{16} ; H_{3,3}$ with the orbit of $\alpha_{1}, \alpha_{2}, \alpha_{3}, \alpha_{4}, \alpha_{5}, \alpha_{6}, \alpha_{7}, \alpha_{8}, \alpha_{11}, \alpha_{12}, \alpha_{15}$ or $\left.\alpha_{16}\right) ; 4 \mathbb{A}_{1}$ with $A_{S} \cong \mathbb{Z} / 8 \mathbb{Z} \times(\mathbb{Z} / 2 \mathbb{Z})^{6}$ $\left(H_{3,1}\right.$ with the orbit of $\alpha_{2}, \alpha_{3}, \alpha_{4}$ or $\alpha_{7} ; H_{3,2}$ with the orbit of $\alpha_{2}$ or $\left.\alpha_{4}\right)$.

$\mathbf{n}=\mathbf{2}, H \cong C_{3}(|H|=3, i=1): \operatorname{rk} N_{H}=12$ and $\left(N_{H}\right)^{*} / N_{H} \cong(\mathbb{Z} / 3 \mathbb{Z})^{6}$. Degenerations of codimension 1 : $\mathbb{A}_{1}$ with $A_{S} \cong \mathbb{Z} / 6 \mathbb{Z} \times(\mathbb{Z} / 3 \mathbb{Z})^{5}\left(H_{2,1}\right.$ with the orbit of $\alpha_{3}, \alpha_{4}, \alpha_{14}, \alpha_{17}, \alpha_{21}$ or $\left.\alpha_{24}\right) ; 3 \mathbb{A}_{1}$ with $A_{S} \cong \mathbb{Z} / 6 \mathbb{Z} \times(\mathbb{Z} / 3 \mathbb{Z})^{4}\left(H_{2,1}\right.$ with the orbit of $\alpha_{1}, \alpha_{2}, \alpha_{5}, \alpha_{6}, \alpha_{12}$ or $\left.\alpha_{15}\right)$.

$\mathbf{n}=\mathbf{1}, H \cong C_{2}(|H|=2, i=1): \operatorname{rk} N_{H}=8$ and $\left(N_{H}\right)^{*} / N_{H} \cong(\mathbb{Z} / 2 \mathbb{Z})^{8}$. Degenerations of codimension 1: $\mathbb{A}_{1}$ with $A_{S} \cong(\mathbb{Z} / 2 \mathbb{Z})^{9}\left(H_{1,1}\right.$ with the orbit of $\alpha_{1}, \alpha_{4}, \alpha_{8}, \alpha_{12}, \alpha_{15}, \alpha_{17}, \alpha_{18}$ or $\left.\alpha_{21}\right) ; 2 \mathbb{A}_{1}$ with $A_{S} \cong \mathbb{Z} / 4 \mathbb{Z} \times(\mathbb{Z} / 2 \mathbb{Z})^{6}\left(H_{1,1}\right.$ with the orbit of $\alpha_{2}, \alpha_{3}, \alpha_{5}, \alpha_{6}, \alpha_{7}, \alpha_{10}, \alpha_{11}$ or $\left.\alpha_{14}\right)$.

Table 22. All possible markings by $N_{22}=N\left(12 A_{2}\right)$ of degenerations of codimension one of non-trivial finite symplectic automorphism groups of Kählerian K3 surfaces:

$\mathbf{n}=\mathbf{7 9}, H \cong \mathfrak{A}_{6}(|H|=360, i=118):$ rk $N_{H}=19$. No degenerations.

$\mathbf{n}=\mathbf{7 0}, H \cong \mathfrak{S}_{5}(|H|=120, i=34):$ rk $N_{H}=19$. No degenerations.

$\mathbf{n}=63, H \cong M_{9}(|H|=72, i=41):$ rk $N_{H}=19$. No degenerations.

$\mathbf{n}=62, H \cong N_{72}(|H|=72, i=40):$ rk $N_{H}=19$. No degenerations.

$\mathbf{n}=55, H \cong \mathfrak{A}_{5}(|H|=60, i=5): \operatorname{rk} N_{H}=18$ and $\left(N_{H}\right)^{*} / N_{H} \cong \mathbb{Z} / 30 \mathbb{Z} \times \mathbb{Z} / 10 \mathbb{Z}$. Degenerations: $\mathbb{A}_{1}$ with $A_{S} \cong \mathbb{Z} / 30 \mathbb{Z} \times \mathbb{Z} / 10 \mathbb{Z} \times \mathbb{Z} / 2 \mathbb{Z}\left(H_{55,1}\right.$ with the orbit of $\alpha_{1,10}$ or $\alpha_{2,10}$; $H_{55,2}$ with the orbit of $\alpha_{1,6}, \alpha_{2,6}, \alpha_{1,8}$ or $\left.\alpha_{2,8}\right) ; 5 \mathbb{A}_{1}$ with $A_{S} \cong \mathbb{Z} / 30 \mathbb{Z} \times(\mathbb{Z} / 2 \mathbb{Z})^{2}\left(H_{55,1}\right.$ with the orbit of $\alpha_{1,6}$ or $\left.\alpha_{2,6}\right) ; 6 \mathbb{A}_{1}$ with $A_{S} \cong \mathbb{Z} / 20 \mathbb{Z} \times \mathbb{Z} / 5 \mathbb{Z}\left(H_{55,1}\right.$ with the orbit of $\alpha_{1,1}$ or $\left.\alpha_{2,1}\right)$; $10 \mathbb{A}_{1}$ with $A_{S} \cong \mathbb{Z} / 60 \mathbb{Z}\left(H_{55,2}\right.$ with the orbit of $\alpha_{1,1}$ or $\left.\alpha_{2,1}\right)$.

$\mathbf{n}=\mathbf{5 4}, H \cong T_{48}(|H|=48, i=29):$ rk $N_{H}=19$. No degenerations.

$\mathbf{n}=\mathbf{4 8}, H \cong \mathfrak{S}_{3,3}(|H|=36, i=10): \operatorname{rk} N_{H}=18,\left(N_{H}\right)^{*} / N_{H} \cong \mathbb{Z} / 18 \mathbb{Z} \times \mathbb{Z} / 6 \mathbb{Z} \times(\mathbb{Z} / 3 \mathbb{Z})^{2}$. Degenerations: $3 \mathbb{A}_{1}$ with $A_{S} \cong \mathbb{Z} / 18 \mathbb{Z} \times(\mathbb{Z} / 6 \mathbb{Z})^{2}\left(H_{48,2}\right.$ with the orbit of $\alpha_{1,6}, \alpha_{2,6}, \alpha_{1,7}$ or $\left.\alpha_{2,7}\right) ; 6 \mathbb{A}_{1}$ with $A_{S} \cong \mathbb{Z} / 36 \mathbb{Z} \times(\mathbb{Z} / 3 \mathbb{Z})^{2}\left(H_{48,2}\right.$ with the orbit of $\alpha_{1,1}$ or $\left.\alpha_{1,2}\right) ; 9 \mathbb{A}_{1}$ with $A_{S} \cong(\mathbb{Z} / 6 \mathbb{Z})^{3}\left(H_{48,1}\right.$ with the orbit of $\alpha_{1,1}$ or $\left.\alpha_{2,1}\right)$.

$\mathbf{n}=\mathbf{4 6}, H \cong 3^{2} C_{4}(|H|=36, i=9): \operatorname{rk} N_{H}=18$ and $\left(N_{H}\right)^{*} / N_{H} \cong \mathbb{Z} / 18 \mathbb{Z} \times \mathbb{Z} / 6 \mathbb{Z} \times \mathbb{Z} / 3 \mathbb{Z}$. Degenerations: $9 \mathbb{A}_{1}$ with $A_{S} \cong(\mathbb{Z} / 6 \mathbb{Z})^{2} \times \mathbb{Z} / 2 \mathbb{Z}\left(H_{46,1}\right.$ with the orbit of $\alpha_{1,1}$ or $\left.\alpha_{2,1}\right) ; 9 \mathbb{A}_{2}$ with $A_{S} \cong \mathbb{Z} / 6 \mathbb{Z} \times \mathbb{Z} / 3 \mathbb{Z}\left(H_{46,2}\right.$ with the orbit of $\left.\alpha_{1,1}\right)$. 
$\mathbf{n}=\mathbf{3 4}, H \cong \mathfrak{S}_{4}(|H|=24, i=12): \operatorname{rk} N_{H}=17$ and $\left(N_{H}\right)^{*} / N_{H} \cong(\mathbb{Z} / 12 \mathbb{Z})^{2} \times \mathbb{Z} / 4 \mathbb{Z}$. Degenerations of codimension 1 : $4 \mathbb{A}_{1}$ with $A_{S} \cong \mathbb{Z} / 24 \mathbb{Z} \times \mathbb{Z} / 12 \mathbb{Z}\left(H_{34,1}\right.$ with the orbit of $\alpha_{1,1}$ or $\left.\alpha_{2,1}\right) ; 6 \mathbb{A}_{2}$ with $A_{S} \cong \mathbb{Z} / 12 \mathbb{Z} \times \mathbb{Z} / 4 \mathbb{Z}\left(H_{34,1}\right.$ with the orbit of $\left.\alpha_{1,2}\right)$.

$\mathbf{n}=32, H \cong H \operatorname{Hol}\left(C_{5}\right) \quad(|H|=20, \quad i=3): \quad \operatorname{rk} N_{H}=18$ and $\left(N_{H}\right)^{*} / N_{H}$ $\cong(\mathbb{Z} / 10 \mathbb{Z})^{2} \times \mathbb{Z} / 5 \mathbb{Z}$. Degenerations: $5 \mathbb{A}_{1}$ with $A_{S} \cong(\mathbb{Z} / 10 \mathbb{Z})^{2} \times \mathbb{Z} / 2 \mathbb{Z}\left(H_{32,1}\right.$ with the orbit of $\alpha_{1,3}$ or $\left.\alpha_{2,3}\right) ; 5 \mathbb{A}_{2}$ with $A_{S} \cong \mathbb{Z} / 10 \mathbb{Z} \times \mathbb{Z} / 5 \mathbb{Z}\left(H_{32,1}\right.$ with the orbit of $\left.\alpha_{1,6}\right)$.

$\mathbf{n}=\mathbf{3 0}, H \cong \mathfrak{A}_{3,3}(|H|=18, i=4): \operatorname{rk} N_{H}=16$ and $\left(N_{H}\right)^{*} / N_{H} \cong \mathbb{Z} / 9 \mathbb{Z} \times(\mathbb{Z} / 3 \mathbb{Z})^{4}$. Degenerations of codimension 1 : $3 \mathbb{A}_{1}$ with $A_{S} \cong \mathbb{Z} / 18 \mathbb{Z} \times(\mathbb{Z} / 3 \mathbb{Z})^{3}\left(H_{30,2}\right.$ with the orbit of $\alpha_{1,1}, \alpha_{2,1}, \alpha_{1,2}, \alpha_{2,2}, \alpha_{1,6}, \alpha_{2,6}, \alpha_{1,7}$ or $\left.\alpha_{2,7}\right) ; 9 \mathbb{A}_{1}$ with $A_{S} \cong \mathbb{Z} / 6 \mathbb{Z} \times(\mathbb{Z} / 3 \mathbb{Z})^{3}\left(H_{30,1}\right.$ with the orbit of $\alpha_{1,1}$ or $\left.\alpha_{2,1}\right)$.

$\mathbf{n}=\mathbf{2 6}, H \cong S D_{16}(|H|=16, i=8): \operatorname{rk} N_{H}=18$ and $\left(N_{H}\right)^{*} / N_{H} \cong(\mathbb{Z} / 8 \mathbb{Z})^{2} \times \mathbb{Z} / 4 \mathbb{Z} \times$ $\mathbb{Z} / 2 \mathbb{Z}$. Degenerations: $2 \mathbb{A}_{2}$ with $A_{S} \cong(\mathbb{Z} / 8 \mathbb{Z})^{2} \times \mathbb{Z} / 2 \mathbb{Z}\left(H_{26,1}\right.$ with the orbit of $\left.\alpha_{1,3}\right) ; 8 \mathbb{A}_{1}$ with $A_{S} \cong \mathbb{Z} / 16 \mathbb{Z} \times \mathbb{Z} / 4 \mathbb{Z} \times \mathbb{Z} / 2 \mathbb{Z}\left(H_{26,1}\right.$ with the orbit of $\alpha_{1,5}$ or $\left.\alpha_{2,5}\right)$.

$\mathbf{n}=\mathbf{1 8}, H \cong D_{12}(|H|=12, i=4): \operatorname{rk} N_{H}=16$ and $\left(N_{H}\right)^{*} / N_{H} \cong(\mathbb{Z} / 6 \mathbb{Z})^{4}$. Degenerations of codimension 1 : $\mathbb{A}_{1}$ with $A_{S} \cong(\mathbb{Z} / 6 \mathbb{Z})^{4} \times \mathbb{Z} / 2 \mathbb{Z}\left(H_{18,1}\right.$ with the orbit of $\alpha_{1,1}$ or $\left.\alpha_{2,1}\right)$; $2 \mathbb{A}_{1}$ with $A_{S} \cong \mathbb{Z} / 12 \mathbb{Z} \times(\mathbb{Z} / 6 \mathbb{Z})^{2} \times \mathbb{Z} / 3 \mathbb{Z}\left(H_{18,1}\right.$ with the orbit of $\alpha_{1,7}$ or $\left.\alpha_{2,7}\right) ; 3 \mathbb{A}_{1}$ with $A_{S} \cong(\mathbb{Z} / 6 \mathbb{Z})^{3} \times(\mathbb{Z} / 2 \mathbb{Z})^{2}\left(H_{18,1}\right.$ with the orbit of $\alpha_{1,2}$ or $\left.\alpha_{2,2}\right) ; 6 \mathbb{A}_{1}$ with $A_{S} \cong \mathbb{Z} / 12 \mathbb{Z} \times(\mathbb{Z} / 6 \mathbb{Z})^{2}$ $\left(H_{18,1}\right.$ with the orbit of $\alpha_{1,5}$ or $\left.\alpha_{2,5}\right)$.

$\mathbf{n}=\mathbf{1 7}, H=\mathfrak{A}_{4}(|H|=12, i=3): \operatorname{rk} N_{H}=16$ and $\left(N_{H}\right)^{*} / N_{H} \cong(\mathbb{Z} / 12 \mathbb{Z})^{2} \times(\mathbb{Z} / 2 \mathbb{Z})^{2}$. Degenerations of codimension 1 : $\mathbb{A}_{1}$ with $A_{S} \cong(\mathbb{Z} / 12 \mathbb{Z})^{2} \times(\mathbb{Z} / 2 \mathbb{Z})^{3}\left(H_{17,1}\right.$ with the orbit of $\alpha_{1,1}, \alpha_{2,1}, \alpha_{1,2}$ or $\left.\alpha_{2,2}\right) ; 4 \mathbb{A}_{1}$ with $A_{S} \cong \mathbb{Z} / 24 \mathbb{Z} \times \mathbb{Z} / 6 \mathbb{Z} \times \mathbb{Z} / 2 \mathbb{Z}\left(H_{17,1}\right.$ with the orbit of $\alpha_{1,3}$ or $\left.\alpha_{2,3}\right) ; 6 \mathbb{A}_{1}$ with $A_{S} \cong \mathbb{Z} / 12 \mathbb{Z} \times(\mathbb{Z} / 4 \mathbb{Z})^{2}\left(H_{17,1}\right.$ with the orbit of $\alpha_{1,5}$ or $\left.\alpha_{2,5}\right)$.

$\mathbf{n}=\mathbf{1 6}, H \cong D_{10}(|H|=10, i=1): \operatorname{rk} N_{H}=16$ and $\left(N_{H}\right)^{*} / N_{H} \cong(\mathbb{Z} / 5 \mathbb{Z})^{4}$. Degenerations of codimension 1 : $\mathbb{A}_{1}$ with $A_{S} \cong \mathbb{Z} / 10 \mathbb{Z} \times(\mathbb{Z} / 5 \mathbb{Z})^{3}\left(H_{16,1}\right.$ with the orbit of $\alpha_{1,1}, \alpha_{2,1}$, $\alpha_{1,2}$ or $\left.\alpha_{2,2}\right) ; 5 \mathbb{A}_{1}$ with $A_{S} \cong \mathbb{Z} / 10 \mathbb{Z} \times(\mathbb{Z} / 5 \mathbb{Z})^{2}\left(H_{16,1}\right.$ with the orbit of $\alpha_{1,3}, \alpha_{2,3}, \alpha_{1,5}$ or $\left.\alpha_{2,5}\right)$.

$\mathbf{n}=\mathbf{1 2}, H \cong Q_{8}(|H|=8, i=4): \operatorname{rk} N_{H}=17,\left(N_{H}\right)^{*} / N_{H} \cong(\mathbb{Z} / 8 \mathbb{Z})^{2} \times(\mathbb{Z} / 2 \mathbb{Z})^{3}$. Degenerations of codimension 1 : $\mathbb{A}_{2}$ with $A_{S} \cong(\mathbb{Z} / 8 \mathbb{Z})^{2} \times(\mathbb{Z} / 2 \mathbb{Z})^{2}\left(H_{12,1}\right.$ with the orbit of $\alpha_{1,5}, \alpha_{1,8}$ or $\left.\alpha_{1,11}\right) ; 8 \mathbb{A}_{1}$ with $A_{S} \cong \mathbb{Z} / 16 \mathbb{Z} \times(\mathbb{Z} / 2 \mathbb{Z})^{3}\left(H_{12,1}\right.$ with the orbit of $\alpha_{1,1}$ or $\left.\alpha_{2,1}\right)$.

$\mathbf{n}=\mathbf{1 0}, H \cong D_{8}(|H|=8, i=3): \operatorname{rk} N_{H}=15$ and $\left(N_{H}\right)^{*} / N_{H} \cong(\mathbb{Z} / 4 \mathbb{Z})^{5}$. Degenerations of codimension 1 : $\mathbb{A}_{1}$ with $A_{S} \cong(\mathbb{Z} / 4 \mathbb{Z})^{5} \times \mathbb{Z} / 2 \mathbb{Z}\left(H_{10,1}\right.$ with the orbit of $\alpha_{1,1}, \alpha_{2,1}, \alpha_{1,2}$ or $\left.\alpha_{2,2}\right) ; 4 \mathbb{A}_{1}$ with $A_{S} \cong \mathbb{Z} / 8 \mathbb{Z} \times(\mathbb{Z} / 4 \mathbb{Z})^{3}\left(H_{10,1}\right.$ with the orbit of $\alpha_{1,3}, \alpha_{2,3}, \alpha_{1,5}$ or $\left.\alpha_{2,5}\right) ; 2 \mathbb{A}_{2}$ with $A_{S} \cong(\mathbb{Z} / 4 \mathbb{Z})^{4}\left(H_{10,1}\right.$ with the orbit of $\left.\alpha_{1,9}\right)$.

$\mathbf{n}=6, H \cong D_{6}(|H|=6, i=1): \operatorname{rk} N_{H}=14,\left(N_{H}\right)^{*} / N_{H} \cong(\mathbb{Z} / 6 \mathbb{Z})^{2} \times(\mathbb{Z} / 3 \mathbb{Z})^{3}$. Degenerations of codimension 1 : $\mathbb{A}_{1}$ with $A_{S} \cong(\mathbb{Z} / 6 \mathbb{Z})^{3} \times(\mathbb{Z} / 3 \mathbb{Z})^{2}\left(H_{6,1}\right.$ with the orbit 
of $\alpha_{1,8}$ or $\alpha_{2,8} ; H_{6,2}$ with the orbit of $\alpha_{1,6}, \alpha_{2,6}, \alpha_{1,8}, \alpha_{2,8}, \alpha_{1,10}$ or $\left.\alpha_{2,10}\right) ; 2 \mathbb{A}_{1}$ with $A_{S} \cong$ $\mathbb{Z} / 12 \mathbb{Z} \times(\mathbb{Z} / 3 \mathbb{Z})^{4}\left(H_{6,1}\right.$ with the orbit of $\alpha_{1,6}$ or $\left.\alpha_{2,6}\right) ; 3 \mathbb{A}_{1}$ with $A_{S} \cong(\mathbb{Z} / 6 \mathbb{Z})^{3} \times \mathbb{Z} / 3 \mathbb{Z}\left(H_{6,2}\right.$ with the orbit of $\alpha_{1,1}$ or $\left.\alpha_{2,1}\right) ; 6 \mathbb{A}_{1}$ with $A_{S} \cong \mathbb{Z} / 12 \mathbb{Z} \times(\mathbb{Z} / 3 \mathbb{Z})^{3}\left(H_{6,2}\right.$ with the orbit of $\alpha_{1,2}$ or $\left.\alpha_{2,2}\right)$.

$\mathbf{n}=4, H \cong C_{4}(|H|=4, i=1): \operatorname{rk} N_{H}=14$ and $\left(N_{H}\right)^{*} / N_{H} \cong(\mathbb{Z} / 4 \mathbb{Z})^{4} \times(\mathbb{Z} / 2 \mathbb{Z})^{2}$. Degenerations of codimension 1 : $\mathbb{A}_{1}$ with $A_{S} \cong(\mathbb{Z} / 4 \mathbb{Z})^{4} \times(\mathbb{Z} / 2 \mathbb{Z})^{3}\left(H_{4,1}\right.$ with the orbit of $\alpha_{1,2}, \alpha_{2,2}, \alpha_{1,5}$ or $\left.\alpha_{2,5}\right) ; \mathbb{A}_{2}$ with $A_{S} \cong(\mathbb{Z} / 4 \mathbb{Z})^{4} \times \mathbb{Z} / 2 \mathbb{Z}\left(H_{4,1}\right.$ with the orbit of $\alpha_{1,1}$ or $\left.\alpha_{1,11}\right)$; $4 \mathbb{A}_{1}$ with $A_{S} \cong \mathbb{Z} / 8 \mathbb{Z} \times(\mathbb{Z} / 4 \mathbb{Z})^{2} \times(\mathbb{Z} / 2 \mathbb{Z})^{2}\left(H_{4,1}\right.$ with the orbit of $\alpha_{1,3}, \alpha_{2,3}, \alpha_{1,6}$ or $\left.\alpha_{2,6}\right)$.

$\mathbf{n}=\mathbf{3}, H \cong C_{2}^{2}(|H|=4, i=2): \operatorname{rk} N_{H}=12$ and $\left(N_{H}\right)^{*} / N_{H} \cong(\mathbb{Z} / 4 \mathbb{Z})^{2} \times(\mathbb{Z} / 2 \mathbb{Z})^{6}$. Degenerations of codimension 1: $\mathbb{A}_{1}$ with $A_{S} \cong(\mathbb{Z} / 4 \mathbb{Z})^{2} \times(\mathbb{Z} / 2 \mathbb{Z})^{7}\left(H_{3,1}\right.$ with the orbit of $\alpha_{1,1}, \alpha_{2,1}, \alpha_{1,2}$ or $\left.\alpha_{2,2}\right) ; 2 \mathbb{A}_{1}$ with $A_{S} \cong(\mathbb{Z} / 4 \mathbb{Z})^{3} \times(\mathbb{Z} / 2 \mathbb{Z})^{4}\left(H_{1,3}\right.$ with the orbit of $\alpha_{1,3}, \alpha_{2,3}$, $\alpha_{1,5}, \alpha_{2,5}, \alpha_{1,7}$ or $\left.\alpha_{2,7}\right) ; 4 \mathbb{A}_{1}$ with $A_{S} \cong \mathbb{Z} / 8 \mathbb{Z} \times(\mathbb{Z} / 2 \mathbb{Z})^{6}\left(H_{3,1}\right.$ with the orbit of $\alpha_{1,6}$ or $\left.\alpha_{2,6}\right)$.

$\mathbf{n}=\mathbf{2}, H \cong C_{3}(|H|=3, i=1): \operatorname{rk} N_{H}=12$ and $\left(N_{H}\right)^{*} / N_{H} \cong(\mathbb{Z} / 3 \mathbb{Z})^{6}$. Degenerations of codimension 1: $\mathbb{A}_{1}$ with $A_{S} \cong \mathbb{Z} / 6 \mathbb{Z} \times(\mathbb{Z} / 3 \mathbb{Z})^{5}\left(H_{2,1}\right.$ with the orbit of $\alpha_{1,6}, \alpha_{2,6}, \alpha_{1,8}, \alpha_{2,8}$, $\alpha_{1,10}$ or $\left.\alpha_{2,10}\right) ; 3 \mathbb{A}_{1}$ with $A_{S} \cong \mathbb{Z} / 6 \mathbb{Z} \times(\mathbb{Z} / 3 \mathbb{Z})^{4}\left(H_{2,1}\right.$ with the orbit of $\alpha_{1,1}, \alpha_{2,1}, \alpha_{1,2}, \alpha_{2,2}$, $\alpha_{1,7}$ or $\left.\alpha_{2,7}\right)$.

$\mathbf{n}=\mathbf{1}, H \cong C_{2}(|H|=2, i=1): \operatorname{rk} N_{H}=8$ and $\left(N_{H}\right)^{*} / N_{H} \cong(\mathbb{Z} / 2 \mathbb{Z})^{8}$. Degenerations of codimension 1: $\mathbb{A}_{1}$ with $A_{S} \cong(\mathbb{Z} / 2 \mathbb{Z})^{9}\left(H_{1,1}\right.$ with the orbit of $\alpha_{1,1}, \alpha_{2,1}, \alpha_{1,2}, \alpha_{2,2}, \alpha_{1,5}, \alpha_{2,5}$, $\alpha_{1,11}$ or $\left.\alpha_{2,11}\right) ; 2 \mathbb{A}_{1}$ with $A_{S} \cong \mathbb{Z} / 4 \mathbb{Z} \times(\mathbb{Z} / 2 \mathbb{Z})^{6}$ ( $H_{1,1}$ with the orbit of $\alpha_{1,3}, \alpha_{2,3}, \alpha_{1,6}, \alpha_{2,6}$, $\alpha_{1,7}, \alpha_{2,7}, \alpha_{1,9}$ or $\left.\alpha_{2,9}\right)$.

Table 21. All possible markings by $N_{21}=N\left(8 A_{3}\right)$ of degenerations of codimension one of non-trivial finite symplectic automorphism groups of Kählerian K3 surfaces:

$$
\begin{aligned}
& \mathbf{n}=\mathbf{7 4}, H \cong L_{2}(7)(|H|=168, i=42): \operatorname{rk} N_{H}=19 . \text { No degenerations. } \\
& \mathbf{n}=\mathbf{5 1}, H \cong C_{2} \times \mathfrak{S}_{4}(|H|=48, i=48): \operatorname{rk} N_{H}=18,\left(N_{H}\right)^{*} / N_{H} \cong(\mathbb{Z} / 12 \mathbb{Z})^{2} \times(\mathbb{Z} / 2 \mathbb{Z})^{2} .
\end{aligned}
$$
Degenerations: $2 \mathbb{A}_{1}$ with $A_{S} \cong(\mathbb{Z} / 12 \mathbb{Z})^{2} \times \mathbb{Z} / 4 \mathbb{Z}\left(H_{51,1}\right.$ with the orbit of $\alpha_{1,1}$ or $\alpha_{1,2} ; H_{51,2}$ with the orbit of $\left.\alpha_{1,1}\right) ; 4 \mathbb{A}_{1}$ with $A_{S} \cong \mathbb{Z} / 24 \mathbb{Z} \times \mathbb{Z} / 6 \mathbb{Z} \times \mathbb{Z} / 2 \mathbb{Z}\left(H_{51,2}\right.$ with the orbit of $\left.\alpha_{2,4}\right) ; 6 \mathbb{A}_{1}$ with $A_{S} \cong \mathbb{Z} / 12 \mathbb{Z} \times(\mathbb{Z} / 4 \mathbb{Z})^{2}\left(H_{51,2}\right.$ with the orbit of $\left.\alpha_{1,2}\right) ; 8 \mathbb{A}_{1}$ with $A_{S} \cong$ $\mathbb{Z} / 12 \mathbb{Z} \times \mathbb{Z} / 6 \mathbb{Z} \times \mathbb{Z} / 2 \mathbb{Z}\left(H_{51,2}\right.$ with the orbit of $\left.\alpha_{1,4}\right) ; 12 \mathbb{A}_{1}$ with $A_{S} \cong \mathbb{Z} / 24 \mathbb{Z} \times(\mathbb{Z} / 2 \mathbb{Z})^{2}$ $\left(H_{51,1}\right.$ with the orbit of $\left.\alpha_{1,3}\right)$.

$\mathbf{n}=\mathbf{3 4}, H \cong \mathfrak{S}_{4}(|H|=24, i=12): \operatorname{rk} N_{H}=17$ and $\left(N_{H}\right)^{*} / N_{H} \cong(\mathbb{Z} / 12 \mathbb{Z})^{2} \times \mathbb{Z} / 4 \mathbb{Z}$. Degenerations of codimension 1 : $\mathbb{A}_{1}$ with $A_{S} \cong(\mathbb{Z} / 12 \mathbb{Z})^{2} \times \mathbb{Z} / 4 \mathbb{Z} \times \mathbb{Z} / 2 \mathbb{Z}\left(H_{34,1}\right.$ with the orbit of $\alpha_{2,1}, \alpha_{1,2}, \alpha_{2,2}$ or $\alpha_{3,2} ; H_{34,2}$ with the orbit of $\alpha_{2,1} ; H_{34,3}$ with the orbit of $\alpha_{1,2}, \alpha_{2,2}$ or 
$\left.\alpha_{3,2}\right) ; 2 \mathbb{A}_{1}$ with $A_{S} \cong(\mathbb{Z} / 12 \mathbb{Z})^{2} \times(\mathbb{Z} / 2 \mathbb{Z})^{2}\left(H_{34,1}\right.$ with the orbit of $\alpha_{1,1} ; H_{34,2}$ with the orbit of $\left.\alpha_{1,1}\right) ; 3 \mathbb{A}_{1}$ with $A_{S} \cong \mathbb{Z} / 12 \mathbb{Z} \times(\mathbb{Z} / 4 \mathbb{Z})^{2} \times \mathbb{Z} / 2 \mathbb{Z}\left(H_{34,2}\right.$ with the orbit of $\alpha_{2,2} ; H_{34,3}$ with the orbit of $\left.\alpha_{2,1}\right) ; 4 \mathbb{A}_{1}$ with $A_{S} \cong \mathbb{Z} / 24 \mathbb{Z} \times \mathbb{Z} / 12 \mathbb{Z}$ ( $H_{34,2}$ with the orbit of $\alpha_{1,4}, \alpha_{2,4}$ or $\alpha_{3,4}$; $H_{34,3}$ with the orbit of $\left.\alpha_{2,4}\right) ; 6 \mathbb{A}_{1}$ with $A_{S} \cong \mathbb{Z} / 12 \mathbb{Z} \times \mathbb{Z} / 4 \mathbb{Z} \times(\mathbb{Z} / 2 \mathbb{Z})^{2}\left(H_{34,1}\right.$ with the orbit of $\alpha_{2,3} ; H_{34,2}$ with the orbit of $\alpha_{1,2} ; H_{34,3}$ with the orbit of $\left.\alpha_{1,1}\right) ; 8 \mathbb{A}_{1}$ with $A_{S} \cong(\mathbb{Z} / 12 \mathbb{Z})^{2}$ ( $H_{34,3}$ with the orbit of $\left.\alpha_{1,4}\right) ; 12 \mathbb{A}_{1}$ with $A_{S} \cong \mathbb{Z} / 24 \mathbb{Z} \times \mathbb{Z} / 4 \mathbb{Z}\left(H_{34,1}\right.$ with the orbit of $\left.\alpha_{1,3}\right)$.

$\mathbf{n}=\mathbf{3 3}, H \cong C_{7} \rtimes C_{3}(|H|=21, i=1)$ : $\operatorname{rk} N_{H}=18$ and $\left(N_{H}\right)^{*} / N_{H} \cong(\mathbb{Z} / 7 \mathbb{Z})^{3}$. Degenerations: $7 \mathbb{A}_{1}$ with $A_{S} \cong \mathbb{Z} / 14 \mathbb{Z} \times \mathbb{Z} / 7 \mathbb{Z}\left(H_{33,1}\right.$ with the orbit of $\alpha_{1,2}, \alpha_{2,2}$ or $\left.\alpha_{3,2}\right)$.

$\mathbf{n}=\mathbf{2 2}, H \cong C_{2} \times D_{8}(|H|=16, i=11): \operatorname{rk} N_{H}=16,\left(N_{H}\right)^{*} / N_{H} \cong(\mathbb{Z} / 4 \mathbb{Z})^{4} \times(\mathbb{Z} / 2 \mathbb{Z})^{2}$. Degenerations of codimension 1: $2 \mathbb{A}_{1}$ with $A_{S} \cong(\mathbb{Z} / 4 \mathbb{Z})^{5}\left(H_{22,1}\right.$ with the orbit of $\alpha_{1,1}$ or $\left.\alpha_{1,2}\right) ; 4 \mathbb{A}_{1}$ with $A_{S} \cong \mathbb{Z} / 8 \mathbb{Z} \times(\mathbb{Z} / 4 \mathbb{Z})^{2} \times(\mathbb{Z} / 2 \mathbb{Z})^{2}\left(H_{22,1}\right.$ with the orbit of $\alpha_{2,3}$ or $\left.\alpha_{1,4}\right) ; 8 \mathbb{A}_{1}$ with $A_{S} \cong(\mathbb{Z} / 4 \mathbb{Z})^{3} \times(\mathbb{Z} / 2 \mathbb{Z})^{2}\left(H_{22,1}\right.$ with the orbit of $\left.\alpha_{1,3}\right)$.

$\mathbf{n}=\mathbf{1 8}, H \cong D_{12}(|H|=12, i=4): \operatorname{rk} N_{H}=16$ and $\left(N_{H}\right)^{*} / N_{H} \cong(\mathbb{Z} / 6 \mathbb{Z})^{4}$. Degenerations of codimension $1: \mathbb{A}_{1}$ with $A_{S} \cong(\mathbb{Z} / 6 \mathbb{Z})^{4} \times \mathbb{Z} / 2 \mathbb{Z}\left(H_{18,1}\right.$ with the orbit of $\alpha_{2,1}$ or $\left.\alpha_{2,2}\right)$; $2 \mathbb{A}_{1}$ with $A_{S} \cong \mathbb{Z} / 12 \mathbb{Z} \times(\mathbb{Z} / 6 \mathbb{Z})^{2} \times \mathbb{Z} / 3 \mathbb{Z}\left(H_{18,1}\right.$ with the orbit of $\alpha_{1,1}$ or $\left.\alpha_{1,2}\right) ; 3 \mathbb{A}_{1}$ with $A_{S} \cong(\mathbb{Z} / 6 \mathbb{Z})^{3} \times(\mathbb{Z} / 2 \mathbb{Z})^{2}\left(H_{18,1}\right.$ with the orbit of $\alpha_{2,3}$ or $\left.\alpha_{2,5}\right) ; 6 \mathbb{A}_{1}$ with $A_{S} \cong \mathbb{Z} / 12 \mathbb{Z} \times(\mathbb{Z} / 6 \mathbb{Z})^{2}$ $\left(H_{18,1}\right.$ with the orbit of $\alpha_{1,3}$ or $\left.\alpha_{1,5}\right)$.

$\mathbf{n}=\mathbf{1 7}, H \cong \mathfrak{A}_{4}(|H|=12, i=3): \operatorname{rk} N_{H}=16$ and $\left(N_{H}\right)^{*} / N_{H} \cong(\mathbb{Z} / 12 \mathbb{Z})^{2} \times(\mathbb{Z} / 2 \mathbb{Z})^{2}$. Degenerations of codimension 1 : $\mathbb{A}_{1}$ with $A_{S} \cong(\mathbb{Z} / 12 \mathbb{Z})^{2} \times(\mathbb{Z} / 2 \mathbb{Z})^{3}\left(H_{17,1}\right.$ with the orbit of $\alpha_{1,1}, \alpha_{2,1}, \alpha_{3,1}, \alpha_{1,5}, \alpha_{2,5}$ or $\alpha_{3,5} ; H_{17,2}$ with the orbit of $\alpha_{1,1}, \alpha_{2,1}$ or $\left.\alpha_{3,1}\right) ; 3 \mathbb{A}_{1}$ with $A_{S} \cong$ $\mathbb{Z} / 12 \mathbb{Z} \times \mathbb{Z} / 4 \mathbb{Z} \times(\mathbb{Z} / 2 \mathbb{Z})^{3}\left(H_{17,2}\right.$ with the orbit of $\left.\alpha_{2,2}\right) ; 4 \mathbb{A}_{1}$ with $A_{S} \cong \mathbb{Z} / 24 \mathbb{Z} \times \mathbb{Z} / 6 \mathbb{Z} \times \mathbb{Z} / 2 \mathbb{Z}$ $\left(H_{17,2}\right.$ with the orbit of $\alpha_{1,3}, \alpha_{2,3}$ or $\left.\alpha_{3,3}\right) ; 6 \mathbb{A}_{1}$ with $A_{S} \cong \mathbb{Z} / 12 \mathbb{Z} \times(\mathbb{Z} / 4 \mathbb{Z})^{2}\left(H_{17,1}\right.$ with the orbit of $\alpha_{2,2} ; H_{17,2}$ with the orbit of $\left.\alpha_{1,2}\right) ; 12 \mathbb{A}_{1}$ with $A_{S} \cong \mathbb{Z} / 24 \mathbb{Z} \times(\mathbb{Z} / 2 \mathbb{Z})^{2}\left(H_{17,1}\right.$ with the orbit of $\left.\alpha_{1,2}\right)$.

$\mathbf{n}=\mathbf{1 0}, H \cong D_{8}(|H|=8, i=3): \operatorname{rk} N_{H}=15$ and $\left(N_{H}\right)^{*} / N_{H} \cong(\mathbb{Z} / 4 \mathbb{Z})^{5}$. Degenerations of codimension $1: \mathbb{A}_{1}$ with $A_{S} \cong(\mathbb{Z} / 4 \mathbb{Z})^{5} \times \mathbb{Z} / 2 \mathbb{Z}$ ( $H_{10,1}$ with the orbit of $\alpha_{1,1}, \alpha_{2,1}, \alpha_{3,1}$ or $\alpha_{2,2} ; H_{10,2}$ with the orbit of $\alpha_{2,1}$ or $\left.\alpha_{2,2}\right) ; 2 \mathbb{A}_{1}$ with $A_{S} \cong(\mathbb{Z} / 4 \mathbb{Z})^{4} \times(\mathbb{Z} / 2 \mathbb{Z})^{2}\left(H_{10,1}\right.$ with the orbit of $\alpha_{1,2}$ or $\alpha_{2,4} ; H_{10,2}$ with the orbit of $\alpha_{1,1}, \alpha_{1,2}$ or $\left.\alpha_{2,4}\right) ; 4 \mathbb{A}_{1}$ with $A_{S} \cong \mathbb{Z} / 8 \mathbb{Z} \times(\mathbb{Z} / 4 \mathbb{Z})^{3}$ ( $H_{10,1}$ with the orbit of $\alpha_{2,3}$ or $\alpha_{1,4} ; H_{10,2}$ with the orbit of $\alpha_{1,3}, \alpha_{2,3}, \alpha_{3,3}$ or $\alpha_{1,4}$ ); $8 \mathbb{A}_{1}$ with $A_{S} \cong(\mathbb{Z} / 4 \mathbb{Z})^{4}\left(H_{10,1}\right.$ with the orbit of $\left.\alpha_{1,3}\right)$.

$\mathbf{n}=\mathbf{9}, H \cong C_{2}^{3}(|H|=8, i=5): \operatorname{rk} N_{H}=14,\left(N_{H}\right)^{*} / N_{H} \cong(\mathbb{Z} / 4 \mathbb{Z})^{2} \times(\mathbb{Z} / 2 \mathbb{Z})^{6}$. Degenerations of codimension 1: $2 \mathbb{A}_{1}$ with $A_{S} \cong(\mathbb{Z} / 4 \mathbb{Z})^{3} \times(\mathbb{Z} / 2 \mathbb{Z})^{4}\left(H_{9,1}\right.$ with the orbit of $\alpha_{1,1}, \alpha_{1,2}, \alpha_{1,4}$ or $\alpha_{1,5} ; H_{9,2}$ with the orbit of $\alpha_{1,1}, \alpha_{2,2}, \alpha_{2,3}, \alpha_{1,5}$ or $\left.\alpha_{2,7}\right) ; 4 \mathbb{A}_{1}$ with $A_{S} \cong \mathbb{Z} / 8 \mathbb{Z} \times(\mathbb{Z} / 2 \mathbb{Z})^{6}\left(H_{9,1}\right.$ with the orbit of $\alpha_{2,3} ; H_{9,2}$ with the orbit of $\alpha_{1,2}, \alpha_{1,3}$ or $\left.\alpha_{1,7}\right)$; $8 \mathbb{A}_{1}$ with $A_{S} \cong \mathbb{Z} / 4 \mathbb{Z} \times(\mathbb{Z} / 2 \mathbb{Z})^{6}\left(H_{9,1}\right.$ with the orbit of $\left.\alpha_{1,3}\right)$. 
$\mathbf{n}=6, H \cong D_{6}(|H|=6, i=1): \operatorname{rk} N_{H}=14$ and $\left(N_{H}\right)^{*} / N_{H} \cong(\mathbb{Z} / 6 \mathbb{Z})^{2} \times(\mathbb{Z} / 3 \mathbb{Z})^{3}$. Degenerations of codimension 1 : $\mathbb{A}_{1}$ with $A_{S} \cong(\mathbb{Z} / 6 \mathbb{Z})^{3} \times(\mathbb{Z} / 3 \mathbb{Z})^{2}\left(H_{6,1}\right.$ with the orbit of $\alpha_{1,1}, \alpha_{2,1}, \alpha_{3,1}$ or $\left.\alpha_{2,2}\right) ; 2 \mathbb{A}_{1}$ with with $A_{S} \cong \mathbb{Z} / 12 \mathbb{Z} \times(\mathbb{Z} / 3 \mathbb{Z})^{4}\left(H_{6,1}\right.$ with the orbit of $\left.\alpha_{1,2}\right)$; $3 \mathbb{A}_{1}$ with $A_{S} \cong(\mathbb{Z} / 6 \mathbb{Z})^{3} \times \mathbb{Z} / 3 \mathbb{Z}\left(H_{6,1}\right.$ with the orbit of $\alpha_{2,3}, \alpha_{1,5}, \alpha_{2,5}$ or $\left.\alpha_{3,5}\right) ; 6 \mathbb{A}_{1}$ with $A_{S} \cong \mathbb{Z} / 12 \mathbb{Z} \times(\mathbb{Z} / 3 \mathbb{Z})^{3}\left(H_{6,1}\right.$ with the orbit of $\left.\alpha_{1,3}\right)$.

$\mathbf{n}=4, H \cong C_{4}(|H|=4, i=1): \operatorname{rk} N_{H}=14$ and $\left(N_{H}\right)^{*} / N_{H} \cong(\mathbb{Z} / 4 \mathbb{Z})^{4} \times(\mathbb{Z} / 2 \mathbb{Z})^{2}$. Degenerations of codimension 1 : $\mathbb{A}_{1}$ with $A_{S} \cong(\mathbb{Z} / 4 \mathbb{Z})^{4} \times(\mathbb{Z} / 2 \mathbb{Z})^{3}\left(H_{4,1}\right.$ with the orbit of $\alpha_{2,1}, \alpha_{1,2}, \alpha_{2,2}$ or $\left.\alpha_{3,2}\right) ; 2 \mathbb{A}_{1}$ with $A_{S} \cong(\mathbb{Z} / 4 \mathbb{Z})^{5}\left(H_{4,1}\right.$ with the orbit of $\alpha_{1,1}$ or $\left.\alpha_{2,3}\right) ; 4 \mathbb{A}_{1}$ with $A_{S} \cong \mathbb{Z} / 8 \mathbb{Z} \times(\mathbb{Z} / 4 \mathbb{Z})^{2} \times(\mathbb{Z} / 2 \mathbb{Z})^{2}\left(H_{4,1}\right.$ with the orbit of $\alpha_{1,3}, \alpha_{1,4}, \alpha_{2,4}$ or $\left.\alpha_{3,4}\right)$.

$\mathbf{n}=\mathbf{3}, H \cong C_{2}^{2}(|H|=4, i=2): \operatorname{rk} N_{H}=12$ and $\left(N_{H}\right)^{*} / N_{H} \cong(\mathbb{Z} / 4 \mathbb{Z})^{2} \times(\mathbb{Z} / 2 \mathbb{Z})^{6}$. Degenerations of codimension 1 : $\mathbb{A}_{1}$ with $A_{S} \cong(\mathbb{Z} / 4 \mathbb{Z})^{2} \times(\mathbb{Z} / 2 \mathbb{Z})^{7}\left(H_{3,1}\right.$ with the orbit of $\alpha_{2,1}, \alpha_{2,2}, \alpha_{2,4}$ or $\alpha_{2,5} ; H_{3,2}$ with the orbit of $\alpha_{1,1}, \alpha_{2,1}, \alpha_{3,1}, \alpha_{2,2}, \alpha_{2,4}$ or $\alpha_{2,5} ; H_{3,3}$ with the orbit of $\alpha_{1,1}, \alpha_{2,1}, \alpha_{3,1}, \alpha_{1,5}, \alpha_{2,5}$ or $\alpha_{3,5} ; H_{3,4}$ with the orbit of $\alpha_{2,1}$ or $\left.\alpha_{2,5}\right) ; 2 \mathbb{A}_{1}$ with $A_{S} \cong(\mathbb{Z} / 4 \mathbb{Z})^{3} \times(\mathbb{Z} / 2 \mathbb{Z})^{4}\left(H_{3,1}\right.$ with the orbit of $\alpha_{1,1}, \alpha_{1,2}, \alpha_{2,3}, \alpha_{1,4}, \alpha_{1,5}$ or $\alpha_{2,7} ; H_{3,2}$ with the orbit of $\alpha_{1,2}, \alpha_{1,4}$ or $\alpha_{1,5} ; H_{3,3}$ with the orbit of $\alpha_{2,2}, \alpha_{2,3}$, or $\alpha_{2,7} ; H_{3,4}$ with the orbit of $\alpha_{1,1}, \alpha_{2,2}, \alpha_{1,3}, \alpha_{2,3}, \alpha_{3,3}, \alpha_{1,5}, \alpha_{1,7}, \alpha_{2,7}$ or $\left.\alpha_{3,7}\right) ; 4 \mathbb{A}_{1}$ with $A_{S} \cong \mathbb{Z} / 8 \mathbb{Z} \times(\mathbb{Z} / 2 \mathbb{Z})^{6}\left(H_{3,1}\right.$ with the orbit of $\alpha_{1,3}$ or $\alpha_{1,7} ; H_{3,2}$ with the orbit of $\alpha_{1,3}, \alpha_{2,3}$ or $\alpha_{3,3} ; H_{3,3}$ with the orbit of $\alpha_{1,2}$, $\alpha_{1,3}$ or $\alpha_{1,7} ; H_{3,4}$ with the orbit of $\left.\alpha_{1,2}\right)$.

$\mathbf{n}=\mathbf{2}, H \cong C_{3}(|H|=3, i=1): \operatorname{rk} N_{H}=12$ and $\left(N_{H}\right)^{*} / N_{H} \cong(\mathbb{Z} / 3 \mathbb{Z})^{6}$. Degenerations of codimension 1 : $\mathbb{A}_{1}$ with $A_{S} \cong \mathbb{Z} / 6 \mathbb{Z} \times(\mathbb{Z} / 3 \mathbb{Z})^{5}\left(H_{2,1}\right.$ with the orbit of $\alpha_{1,1}, \alpha_{2,1}, \alpha_{3,1}, \alpha_{1,2}$, $\alpha_{2,2}$ or $\left.\alpha_{3,2}\right) ; 3 \mathbb{A}_{1}$ with $A_{S} \cong \mathbb{Z} / 6 \mathbb{Z} \times(\mathbb{Z} / 3 \mathbb{Z})^{4}\left(H_{2,1}\right.$ with the orbit of $\alpha_{1,3}, \alpha_{2,3}, \alpha_{3,3}, \alpha_{1,5}, \alpha_{2,5}$ or $\left.\alpha_{3,5}\right)$.

$\mathbf{n}=\mathbf{1}, H \cong C_{2}(|H|=2, i=1): \operatorname{rk} N_{H}=8$ and $\left(N_{H}\right)^{*} / N_{H} \cong(\mathbb{Z} / 2 \mathbb{Z})^{8}$. Degenerations of codimension 1 : $\mathbb{A}_{1}$ with $A_{S} \cong(\mathbb{Z} / 2 \mathbb{Z})^{9}\left(H_{1,1}\right.$ with the orbit of $\alpha_{2,1}, \alpha_{2,2}, \alpha_{2,3}, \alpha_{2,4}, \alpha_{2,5}$, $\alpha_{2,6}, \alpha_{2,7}$ or $\alpha_{2,8} ; H_{1,2}$ with the orbit of $\alpha_{1,1}, \alpha_{2,1}, \alpha_{3,1}, \alpha_{2,2}, \alpha_{2,4}, \alpha_{1,5}, \alpha_{2,5}$ or $\left.\alpha_{3,5}\right) ; 2 \mathbb{A}_{1}$ with $A_{S} \cong \mathbb{Z} / 4 \mathbb{Z} \times(\mathbb{Z} / 2 \mathbb{Z})^{6}\left(H_{1,1}\right.$ with the orbit of $\alpha_{1,1}, \alpha_{1,2}, \alpha_{1,3}, \alpha_{1,4}, \alpha_{1,5}, \alpha_{1,6}, \alpha_{1,7}$ or $\alpha_{1,8} ; H_{1,2}$ with the orbit of $\alpha_{1,2}, \alpha_{1,3}, \alpha_{2,3}, \alpha_{3,3}, \alpha_{1,4}, \alpha_{1,7}, \alpha_{2,7}$ or $\left.\alpha_{3,7}\right)$.

Table 20. All possible markings by $N_{20}=N\left(6 A_{4}\right)$ of degenerations of codimension one of non-trivial finite symplectic automorphism groups of Kählerian K3 surfaces:

$\mathbf{n}=\mathbf{3 2}, H \cong \operatorname{Hol}\left(C_{5}\right)(|H|=20, i=3): \operatorname{rk} N_{H}=18$ and $\left(N_{H}\right)^{*} / N_{H} \cong(\mathbb{Z} / 10 \mathbb{Z})^{2} \times \mathbb{Z} / 5 \mathbb{Z}$. Degenerations: $2 \mathbb{A}_{1}$ with $A_{S} \cong \mathbb{Z} / 20 \mathbb{Z} \times(\mathbb{Z} / 5 \mathbb{Z})^{2}\left(H_{32,2}\right.$ with the orbit of $\left.\alpha_{1,1}\right) ; 5 \mathbb{A}_{1}$ with $A_{S} \cong(\mathbb{Z} / 10 \mathbb{Z})^{2} \times \mathbb{Z} / 2 \mathbb{Z}\left(H_{32,2}\right.$ with the orbit of $\alpha_{1,2}, \alpha_{2,2}, \alpha_{3,2}$ or $\left.\alpha_{4,2}\right) ; 10 \mathbb{A}_{1}$ with $A_{S} \cong$ 
$\mathbb{Z} / 20 \mathbb{Z} \times \mathbb{Z} / 5 \mathbb{Z}\left(H_{32,1}\right.$ with the orbit of $\left.\alpha_{1,2}\right) ; 5 \mathbb{A}_{2}$ with $A_{S} \cong \mathbb{Z} / 10 \mathbb{Z} \times \mathbb{Z} / 5 \mathbb{Z}\left(H_{32,1}\right.$ with the orbit of $\left.\alpha_{2,2}\right)$.

$\mathbf{n}=\mathbf{1 6}, H \cong D_{10}(|H|=10, i=1): \operatorname{rk} N_{H}=16$ and $\left(N_{H}\right)^{*} / N_{H} \cong(\mathbb{Z} / 5 \mathbb{Z})^{4}$. Degenerations of codimension $1: \mathbb{A}_{1}$ with $A_{S} \cong \mathbb{Z} / 10 \mathbb{Z} \times(\mathbb{Z} / 5 \mathbb{Z})^{3}\left(H_{16,1}\right.$ with the orbit of $\alpha_{1,1}, \alpha_{2,1}$, $\alpha_{3,1}$ or $\left.\alpha_{4,1}\right) ; 5 \mathbb{A}_{1}$ with $A_{S} \cong \mathbb{Z} / 10 \mathbb{Z} \times(\mathbb{Z} / 5 \mathbb{Z})^{2}\left(H_{16,1}\right.$ with the orbit of $\alpha_{1,2}, \alpha_{2,2}, \alpha_{3,2}$ or $\left.\alpha_{4,2}\right)$.

$\mathbf{n}=4, H \cong C_{4}(|H|=4, i=1): \operatorname{rk} N_{H}=14$ and $\left(N_{H}\right)^{*} / N_{H} \cong(\mathbb{Z} / 4 \mathbb{Z})^{4} \times(\mathbb{Z} / 2 \mathbb{Z})^{2}$. Degenerations of codimension 1 : $\mathbb{A}_{1}$ with $A_{S} \cong(\mathbb{Z} / 4 \mathbb{Z})^{4} \times(\mathbb{Z} / 2 \mathbb{Z})^{3}\left(H_{4,1}\right.$ with the orbit of $\alpha_{1,1}, \alpha_{2,1}, \alpha_{3,1}$ or $\left.\alpha_{4,1}\right) ; 2 \mathbb{A}_{1}$ with $A_{S} \cong(\mathbb{Z} / 4 \mathbb{Z})^{5}\left(H_{4,1}\right.$ with the orbit of $\left.\alpha_{1,2}\right)$; $\mathbb{A}_{2}$ with $A_{S} \cong(\mathbb{Z} / 4 \mathbb{Z})^{4} \times \mathbb{Z} / 2 \mathbb{Z}\left(H_{4,1}\right.$ with the orbit of $\left.\alpha_{2,2}\right) ; 4 \mathbb{A}_{1}$ with $A_{S} \cong \mathbb{Z} / 8 \mathbb{Z} \times(\mathbb{Z} / 4 \mathbb{Z})^{2} \times(\mathbb{Z} / 2 \mathbb{Z})^{2}$ ( $H_{4,1}$ with the orbit of $\alpha_{1,3}, \alpha_{2,3}, \alpha_{3,3}$ or $\alpha_{4,3}$ ).

$\mathbf{n}=\mathbf{1}, H \cong C_{2}(|H|=2, i=1)$ : rk $N_{H}=8$ and $\left(N_{H}\right)^{*} / N_{H} \cong(\mathbb{Z} / 2 \mathbb{Z})^{8}$. Degenerations of codimension $1: \mathbb{A}_{1}$ with $A_{S} \cong(\mathbb{Z} / 2 \mathbb{Z})^{9}\left(H_{1,1}\right.$ with the orbit of $\alpha_{1,1}, \alpha_{2,1}, \alpha_{3,1}, \alpha_{4,1}, \alpha_{1,2}, \alpha_{2,2}$, $\alpha_{3,2}$ or $\left.\alpha_{4,2}\right) ; 2 \mathbb{A}_{1}$ with $A_{S} \cong \mathbb{Z} / 4 \mathbb{Z} \times(\mathbb{Z} / 2 \mathbb{Z})^{6}\left(H_{1,1}\right.$ with the orbit of $\alpha_{1,3}, \alpha_{2,3}, \alpha_{3,3}, \alpha_{4,3}$, $\alpha_{1,4}, \alpha_{2,4}, \alpha_{3,4}$ or $\left.\alpha_{4,4}\right)$.

Table 19. All possible markings by $N_{19}=N\left(6 D_{4}\right)$ of degenerations of codimension one of non-trivial finite symplectic automorphism groups of Kählerian K3 surfaces:

$\mathbf{n}=\mathbf{7 0}, H \cong \mathfrak{S}_{5}(|H|=120, i=34):$ rk $N_{H}=19$. No degenerations.

$\mathbf{n}=\mathbf{6 1}, H \cong \mathfrak{A}_{4,3}(|H|=72, i=43): \operatorname{rk} N_{H}=18$ and $\left(N_{H}\right)^{*} / N_{H} \cong(\mathbb{Z} / 12 \mathbb{Z})^{2} \times \mathbb{Z} / 3 \mathbb{Z}$. Degenerations: $3 \mathbb{A}_{1}$ with $A_{S} \cong(\mathbb{Z} / 12 \mathbb{Z})^{2} \times \mathbb{Z} / 2 \mathbb{Z}\left(H_{61,1}\right.$ with the orbit of $\alpha_{1,5}$ or $\left.\alpha_{1,6}\right) ; 12 \mathbb{A}_{1}$ with $A_{S} \cong \mathbb{Z} / 24 \mathbb{Z} \times \mathbb{Z} / 3 \mathbb{Z}\left(H_{61,1}\right.$ with the orbit of $\left.\alpha_{1,1}\right)$.

$\mathbf{n}=55, H \cong \mathfrak{A}_{5}(|H|=60, i=5): \operatorname{rk} N_{H}=18$ and $\left(N_{H}\right)^{*} / N_{H} \cong \mathbb{Z} / 30 \mathbb{Z} \times \mathbb{Z} / 10 \mathbb{Z}$. Degenerations: $\mathbb{A}_{1}$ with $A_{S} \cong \mathbb{Z} / 30 \mathbb{Z} \times \mathbb{Z} / 10 \mathbb{Z} \times \mathbb{Z} / 2 \mathbb{Z}\left(H_{55,1}\right.$ with the orbit of $\alpha_{1,4}, \alpha_{2,4}$ $\alpha_{3,4}$ or $\left.\alpha_{4,4}\right) ; 5 \mathbb{A}_{1}$ with $A_{S} \cong \mathbb{Z} / 30 \mathbb{Z} \times(\mathbb{Z} / 2 \mathbb{Z})^{2}\left(H_{55,1}\right.$ with the orbit of $\left.\alpha_{2,1}\right) ; 15 \mathbb{A}_{1}$ with $A_{S} \cong \mathbb{Z} / 10 \mathbb{Z} \times(\mathbb{Z} / 2 \mathbb{Z})^{2}\left(H_{55,1}\right.$ with the orbit of $\left.\alpha_{1,1}\right)$.

$\mathbf{n}=\mathbf{4 8}, H \cong \mathfrak{S}_{3,3}(|H|=36, i=10): \operatorname{rk} N_{H}=18,\left(N_{H}\right)^{*} / N_{H} \cong \mathbb{Z} / 18 \mathbb{Z} \times \mathbb{Z} / 6 \mathbb{Z} \times(\mathbb{Z} / 3 \mathbb{Z})^{2}$. Degenerations: $3 \mathbb{A}_{1}$ with $A_{S} \cong \mathbb{Z} / 18 \mathbb{Z} \times(\mathbb{Z} / 6 \mathbb{Z})^{2}\left(H_{48,1}\right.$ with the orbit of $\alpha_{1,3}$ or $\left.\alpha_{2,4}\right) ; 6 \mathbb{A}_{1}$ with $A_{S} \cong \mathbb{Z} / 36 \mathbb{Z} \times(\mathbb{Z} / 3 \mathbb{Z})^{2}\left(H_{48,1}\right.$ with the orbit of $\left.\alpha_{1,1}\right) ; 9 \mathbb{A}_{1}$ with $A_{S} \cong(\mathbb{Z} / 6 \mathbb{Z})^{3}\left(H_{48,1}\right.$ with the orbit of $\left.\alpha_{1,4}\right)$.

$\mathbf{n}=\mathbf{3 4}, H \cong \mathfrak{S}_{4}(|H|=24, i=12): \operatorname{rk} N_{H}=17$ and $\left(N_{H}\right)^{*} / N_{H} \cong(\mathbb{Z} / 12 \mathbb{Z})^{2} \times \mathbb{Z} / 4 \mathbb{Z}$. Degenerations of codimension $1: \mathbb{A}_{1}$ with $A_{S} \cong(\mathbb{Z} / 12 \mathbb{Z})^{2} \times \mathbb{Z} / 4 \mathbb{Z} \times \mathbb{Z} / 2 \mathbb{Z}\left(H_{34,1}\right.$ with the orbit of $\alpha_{2,3}$ or $\alpha_{2,4} ; H_{34,2}$ with the orbit of $\alpha_{2,3}, \alpha_{1,4}$ or $\left.\alpha_{2,4}\right) ; 2 \mathbb{A}_{1}$ with $A_{S} \cong(\mathbb{Z} / 12 \mathbb{Z})^{2} \times(\mathbb{Z} / 2 \mathbb{Z})^{2}$ $\left(H_{34,2}\right.$ with the orbit of $\left.\alpha_{3,4}\right) ; 3 \mathbb{A}_{1}$ with $A_{S} \cong \mathbb{Z} / 12 \mathbb{Z} \times(\mathbb{Z} / 4 \mathbb{Z})^{2} \times \mathbb{Z} / 2 \mathbb{Z}\left(H_{34,1}\right.$ with the orbit 
of $\alpha_{1,3}$ or $\alpha_{1,4} ; H_{34,2}$ with the orbit of $\left.\alpha_{1,3}\right) ; 4 \mathbb{A}_{1}$ with $A_{S} \cong \mathbb{Z} / 24 \mathbb{Z} \times \mathbb{Z} / 12 \mathbb{Z}$ ( $H_{34,1}$ with the orbit of $\alpha_{2,1}$ or $\alpha_{4,1} ; H_{34,2}$ with the orbit of $\left.\alpha_{2,1}\right) ; 8 \mathbb{A}_{1}$ with $A_{S} \cong(\mathbb{Z} / 12 \mathbb{Z})^{2}\left(H_{34,1}\right.$ with the orbit of $\left.\alpha_{1,1}\right) ; 12 \mathbb{A}_{1}$ with $A_{S} \cong \mathbb{Z} / 24 \mathbb{Z} \times \mathbb{Z} / 4 \mathbb{Z}\left(H_{34,2}\right.$ with the orbit of $\left.\alpha_{1,1}\right)$.

$\mathbf{n}=\mathbf{3 2}, H \cong \operatorname{Hol}\left(C_{5}\right)(|H|=20, i=3): \operatorname{rk} N_{H}=18$ and $\left(N_{H}\right)^{*} / N_{H} \cong(\mathbb{Z} / 10 \mathbb{Z})^{2} \times \mathbb{Z} / 5 \mathbb{Z}$. Degenerations: $2 \mathbb{A}_{1}$ with $A_{S} \cong \mathbb{Z} / 20 \mathbb{Z} \times(\mathbb{Z} / 5 \mathbb{Z})^{2}\left(H_{32,1}\right.$ with the orbit of $\left.\alpha_{3,3}\right) ; 5 \mathbb{A}_{1}$ with $A_{S} \cong(\mathbb{Z} / 10 \mathbb{Z})^{2} \times \mathbb{Z} / 2 \mathbb{Z}\left(H_{32,1}\right.$ with the orbit of $\alpha_{1,1}$ or $\left.\alpha_{2,1}\right) ; 10 \mathbb{A}_{1}$ with $A_{S} \cong \mathbb{Z} / 20 \mathbb{Z} \times \mathbb{Z} / 5 \mathbb{Z}$ $\left(H_{32,1}\right.$ with the orbit of $\left.\alpha_{3,1}\right)$.

$\mathbf{n}=\mathbf{3 0}, H \cong \mathfrak{A}_{3,3}(|H|=18, i=4): \operatorname{rk} N_{H}=16$ and $\left(N_{H}\right)^{*} / N_{H} \cong \mathbb{Z} / 9 \mathbb{Z} \times(\mathbb{Z} / 3 \mathbb{Z})^{4}$. Degenerations of codimension 1: $3 \mathbb{A}_{1}$ with $A_{S} \cong \mathbb{Z} / 18 \mathbb{Z} \times(\mathbb{Z} / 3 \mathbb{Z})^{3}\left(H_{30,1}\right.$ with the orbit of $\alpha_{2,1}, \alpha_{1,4}, \alpha_{1,5}$ or $\left.\alpha_{1,6}\right) ; 9 \mathbb{A}_{1}$ with $A_{S} \cong \mathbb{Z} / 6 \mathbb{Z} \times(\mathbb{Z} / 3 \mathbb{Z})^{3}\left(H_{30,1}\right.$ with the orbit of $\left.\alpha_{1,1}\right)$.

$\mathbf{n}=\mathbf{1 8}, H \cong D_{12}(|H|=12, i=4): \operatorname{rk} N_{H}=16$ and $\left(N_{H}\right)^{*} / N_{H} \cong(\mathbb{Z} / 6 \mathbb{Z})^{4}$. Degenerations of codimension 1 : $\mathbb{A}_{1}$ with $A_{S} \cong(\mathbb{Z} / 6 \mathbb{Z})^{4} \times \mathbb{Z} / 2 \mathbb{Z}\left(H_{18,1}\right.$ with the orbit of $\alpha_{2,3}$ or $\alpha_{2,4}$; $H_{18,2}$ with the orbit of $\alpha_{2,3}$ or $\left.\alpha_{4,3}\right) ; 2 \mathbb{A}_{1}$ with $A_{S} \cong \mathbb{Z} / 12 \mathbb{Z} \times(\mathbb{Z} / 6 \mathbb{Z})^{2} \times \mathbb{Z} / 3 \mathbb{Z}\left(H_{18,1}\right.$ with the orbit of $\alpha_{2,1}$ or $\alpha_{2,5} ; H_{18,2}$ with the orbit of $\alpha_{1,3}$ or $\left.\alpha_{2,4}\right) ; 3 \mathbb{A}_{1}$ with $A_{S} \cong(\mathbb{Z} / 6 \mathbb{Z})^{3} \times(\mathbb{Z} / 2 \mathbb{Z})^{2}$ ( $H_{18,1}$ with the orbit of $\alpha_{1,3}$ or $\alpha_{1,4} ; H_{18,2}$ with the orbit of $\alpha_{2,1}$ or $\left.\alpha_{3,1}\right) ; 6 \mathbb{A}_{1}$ with $A_{S} \cong$ $\mathbb{Z} / 12 \mathbb{Z} \times(\mathbb{Z} / 6 \mathbb{Z})^{2}\left(H_{18,1}\right.$ with the orbit of $\alpha_{1,1}$ or $\alpha_{1,5} ; H_{18,2}$ with the orbit of $\alpha_{1,1}$ or $\left.\alpha_{1,4}\right)$.

$\mathbf{n}=\mathbf{1 7}, H \cong \mathfrak{A}_{4}(|H|=12, i=3): \operatorname{rk} N_{H}=16$ and $\left(N_{H}\right)^{*} / N_{H} \cong(\mathbb{Z} / 12 \mathbb{Z})^{2} \times(\mathbb{Z} / 2 \mathbb{Z})^{2}$. Degenerations of codimension $1: \mathbb{A}_{1}$ with $A_{S} \cong(\mathbb{Z} / 12 \mathbb{Z})^{2} \times(\mathbb{Z} / 2 \mathbb{Z})^{3}\left(H_{17,1}\right.$ with the orbit of $\alpha_{2,3}$ or $\alpha_{2,4} ; H_{17,2}$ with the orbit of $\alpha_{2,3}, \alpha_{1,4}, \alpha_{2,4}, \alpha_{3,4}$ or $\left.\alpha_{4,4}\right) ; 3 \mathbb{A}_{1}$ with $A_{S} \cong \mathbb{Z} / 12 \mathbb{Z} \times$ $\mathbb{Z} / 4 \mathbb{Z} \times(\mathbb{Z} / 2 \mathbb{Z})^{3}\left(H_{17,1}\right.$ with the orbit of $\alpha_{1,3}$ or $\alpha_{1,4} ; H_{17,2}$ with the orbit of $\left.\alpha_{1,3}\right) ; 4 \mathbb{A}_{1}$ with $A_{S} \cong \mathbb{Z} / 24 \mathbb{Z} \times \mathbb{Z} / 6 \mathbb{Z} \times \mathbb{Z} / 2 \mathbb{Z}\left(H_{17,1}\right.$ with the orbit of $\alpha_{1,1}, \alpha_{2,1}, \alpha_{3,1}$ or $\alpha_{4,1} ; H_{17,2}$ with the orbit of $\left.\alpha_{2,1}\right) ; 12 \mathbb{A}_{1}$ with $A_{S} \cong \mathbb{Z} / 24 \mathbb{Z} \times(\mathbb{Z} / 2 \mathbb{Z})^{2}\left(H_{17,2}\right.$ with the orbit of $\left.\alpha_{1,1}\right)$.

$\mathbf{n}=\mathbf{1 6}, H \cong D_{10}(|H|=10, i=1): \operatorname{rk} N_{H}=16$ and $\left(N_{H}\right)^{*} / N_{H} \cong(\mathbb{Z} / 5 \mathbb{Z})^{4}$. Degenerations of codimension $1: \mathbb{A}_{1}$ with $A_{S} \cong \mathbb{Z} / 10 \mathbb{Z} \times(\mathbb{Z} / 5 \mathbb{Z})^{3}\left(H_{16,1}\right.$ with the orbit of $\alpha_{1,3}, \alpha_{2,3}$, $\alpha_{3,3}$ or $\left.\alpha_{4,3}\right) ; 5 \mathbb{A}_{1}$ with $A_{S} \cong \mathbb{Z} / 10 \mathbb{Z} \times(\mathbb{Z} / 5 \mathbb{Z})^{2}\left(H_{16,1}\right.$ with the orbit of $\alpha_{1,1}, \alpha_{2,1}, \alpha_{3,1}$ or $\left.\alpha_{4,1}\right)$.

$\mathbf{n}=\mathbf{1 0}, H \cong D_{8}(|H|=8, i=3): \operatorname{rk} N_{H}=15$ and $\left(N_{H}\right)^{*} / N_{H} \cong(\mathbb{Z} / 4 \mathbb{Z})^{5}$. Degenerations of codimension 1 : $\mathbb{A}_{1}$ with $A_{S} \cong(\mathbb{Z} / 4 \mathbb{Z})^{5} \times \mathbb{Z} / 2 \mathbb{Z}\left(H_{10,1}\right.$ with the orbit of $\alpha_{1,3}, \alpha_{2,3}, \alpha_{2,4}$ or $\left.\alpha_{3,4}\right) ; 2 \mathbb{A}_{1}$ with $A_{S} \cong(\mathbb{Z} / 4 \mathbb{Z})^{4} \times(\mathbb{Z} / 2 \mathbb{Z})^{2}\left(H_{10,1}\right.$ with the orbit of $\alpha_{3,3}$ or $\left.\alpha_{1,4}\right) ; 4 \mathbb{A}_{1}$ with $A_{S} \cong \mathbb{Z} / 8 \mathbb{Z} \times(\mathbb{Z} / 4 \mathbb{Z})^{3}\left(H_{10,1}\right.$ with the orbit of $\alpha_{2,1}$ or $\left.\alpha_{4,1}\right) ; 8 \mathbb{A}_{1}$ with $A_{S} \cong(\mathbb{Z} / 4 \mathbb{Z})^{4}\left(H_{10,1}\right.$ with the orbit of $\left.\alpha_{1,1}\right)$.

$\mathbf{n}=\mathbf{6}, H \cong D_{6}(|H|=6, i=1): \operatorname{rk} N_{H}=14$ and $\left(N_{H}\right)^{*} / N_{H} \cong(\mathbb{Z} / 6 \mathbb{Z})^{2} \times(\mathbb{Z} / 3 \mathbb{Z})^{3}$. Degenerations of codimension 1 : $\mathbb{A}_{1}$ with $A_{S} \cong(\mathbb{Z} / 6 \mathbb{Z})^{3} \times(\mathbb{Z} / 3 \mathbb{Z})^{2}\left(H_{6,1}\right.$ with the orbit of $\alpha_{2,3}, \alpha_{2,4}, \alpha_{2,5}$ or $\alpha_{2,6} ; H_{6,2}$ with the orbit of $\alpha_{2,4}, \alpha_{4,4}, \alpha_{2,5}$ or $\alpha_{2,6} ; H_{6,3}$ with the orbit of $\alpha_{1,3}, \alpha_{2,3}, \alpha_{3,3}$ or $\left.\alpha_{4,3}\right) ; 2 \mathbb{A}_{1}$ with with $A_{S} \cong \mathbb{Z} / 12 \mathbb{Z} \times(\mathbb{Z} / 3 \mathbb{Z})^{4}\left(H_{6,1}\right.$ with the orbit of $\alpha_{2,1}$; $H_{6,2}$ with the orbit of $\alpha_{1,4} ; H_{6,3}$ with the orbit of $\left.\alpha_{2,1}\right) ; 3 \mathbb{A}_{1}$ with $A_{S} \cong(\mathbb{Z} / 6 \mathbb{Z})^{3} \times \mathbb{Z} / 3 \mathbb{Z}\left(H_{6,1}\right.$ 
with the orbit of $\alpha_{1,3}, \alpha_{1,4}, \alpha_{1,5}$ or $\alpha_{1,6} ; H_{6,2}$ with the orbit of $\alpha_{2,1}, \alpha_{4,1}, \alpha_{1,5}$ or $\alpha_{1,6} ; H_{6,3}$ with the orbit of $\alpha_{1,4}, \alpha_{2,4}, \alpha_{3,4}$ or $\left.\alpha_{4,4}\right) ; 6 \mathbb{A}_{1}$ with $A_{S} \cong \mathbb{Z} / 12 \mathbb{Z} \times(\mathbb{Z} / 3 \mathbb{Z})^{3}\left(H_{6,1}\right.$ with the orbit of $\alpha_{1,1} ; H_{6,2}$ with the orbit of $\alpha_{1,1} ; H_{6,3}$ with the orbit of $\left.\alpha_{1,1}\right)$.

$\mathbf{n}=4, H \cong C_{4}(|H|=4, i=1): \operatorname{rk} N_{H}=14$ and $\left(N_{H}\right)^{*} / N_{H} \cong(\mathbb{Z} / 4 \mathbb{Z})^{4} \times(\mathbb{Z} / 2 \mathbb{Z})^{2}$. Degenerations of codimension 1: $\mathbb{A}_{1}$ with $A_{S} \cong(\mathbb{Z} / 4 \mathbb{Z})^{4} \times(\mathbb{Z} / 2 \mathbb{Z})^{3}\left(H_{4,1}\right.$ with the orbit of $\alpha_{2,3}, \alpha_{3,3} \alpha_{1,4}$ or $\left.\alpha_{2,4}\right) ; 2 \mathbb{A}_{1}$ with with $A_{S} \cong(\mathbb{Z} / 4 \mathbb{Z})^{5}\left(H_{4,1}\right.$ with the orbit of $\alpha_{1,3}$ or $\left.\alpha_{3,4}\right) ; 4 \mathbb{A}_{1}$ with $A_{S} \cong \mathbb{Z} / 8 \mathbb{Z} \times(\mathbb{Z} / 4 \mathbb{Z})^{2} \times(\mathbb{Z} / 2 \mathbb{Z})^{2}\left(H_{4,1}\right.$ with the orbit of $\alpha_{1,1}, \alpha_{2,1}, \alpha_{3,1}$ or $\left.\alpha_{4,1}\right)$.

$\mathbf{n}=\mathbf{3}, H \cong C_{2}^{2}(|H|=4, i=2): \operatorname{rk} N_{H}=12$ and $\left(N_{H}\right)^{*} / N_{H} \cong(\mathbb{Z} / 4 \mathbb{Z})^{2} \times(\mathbb{Z} / 2 \mathbb{Z})^{6}$. Degenerations of codimension 1: $\mathbb{A}_{1}$ with $A_{S} \cong(\mathbb{Z} / 4 \mathbb{Z})^{2} \times(\mathbb{Z} / 2 \mathbb{Z})^{7}\left(H_{3,1}\right.$ with the orbit of $\alpha_{2,5}, \alpha_{4,5}, \alpha_{2,6}$ or $\alpha_{4,6} ; H_{3,2}$ with the orbit of $\alpha_{1,3}, \alpha_{2,3}, \alpha_{3,3}, \alpha_{4,3}, \alpha_{1,4}, \alpha_{3,4}$ or $\left.\alpha_{4,4}\right) ; 2 \mathbb{A}_{1}$ with $A_{S} \cong(\mathbb{Z} / 4 \mathbb{Z})^{3} \times(\mathbb{Z} / 2 \mathbb{Z})^{4}\left(H_{3,1}\right.$ with the orbit of $\alpha_{2,1}, \alpha_{4,1}, \alpha_{2,3}, \alpha_{4,3}, \alpha_{1,5}$ or $\alpha_{1,6} ; H_{3,3}$ with the orbit of $\alpha_{1,1}, \alpha_{2,1}, \alpha_{3,1}, \alpha_{4,1}, \alpha_{1,3}, \alpha_{2,3}, \alpha_{3,3}, \alpha_{4,3}, \alpha_{1,5}, \alpha_{2,5}, \alpha_{3,5}$ or $\left.\alpha_{4,5}\right) ; 4 \mathbb{A}_{1}$ with $A_{S} \cong \mathbb{Z} / 8 \mathbb{Z} \times(\mathbb{Z} / 2 \mathbb{Z})^{6}\left(H_{3,1}\right.$ with the orbit of $\alpha_{1,1}$ or $\alpha_{1,3} ; H_{3,2}$ with the orbit of $\alpha_{1,1} \alpha_{2,1}$, $\alpha_{3,1}$ or $\left.\alpha_{4,1}\right)$.

$\mathbf{n}=\mathbf{2}, H \cong C_{3}(|H|=3, i=1): \operatorname{rk} N_{H}=12$ and $\left(N_{H}\right)^{*} / N_{H} \cong(\mathbb{Z} / 3 \mathbb{Z})^{6}$. Degenerations of codimension 1 : $\mathbb{A}_{1}$ with $A_{S} \cong \mathbb{Z} / 6 \mathbb{Z} \times(\mathbb{Z} / 3 \mathbb{Z})^{5}\left(H_{2,1}\right.$ with the orbit of $\alpha_{2,1}, \alpha_{2,2}, \alpha_{2,3}$, $\alpha_{2,4}, \alpha_{2,5}$ or $\alpha_{2,6} ; H_{2,2}$ with the orbit of $\alpha_{2,3}, \alpha_{1,4}, \alpha_{2,4}, \alpha_{3,4}, \alpha_{4,4}$ or $\left.\alpha_{2,5}\right) ; 3 \mathbb{A}_{1}$ with with $A_{S} \cong \mathbb{Z} / 6 \mathbb{Z} \times(\mathbb{Z} / 3 \mathbb{Z})^{4}\left(H_{2,1}\right.$ with the orbit of $\alpha_{1,1}, \alpha_{1,2}, \alpha_{1,3}, \alpha_{1,4}, \alpha_{1,5}$ or $\alpha_{1,6} ; H_{2,2}$ with the orbit of $\alpha_{1,1}, \alpha_{2,1}, \alpha_{3,1}, \alpha_{4,1}, \alpha_{1,3}$ or $\left.\alpha_{1,5}\right)$.

$\mathbf{n}=\mathbf{1}, H \cong C_{2}(|H|=2, i=1): \operatorname{rk} N_{H}=8$ and $\left(N_{H}\right)^{*} / N_{H} \cong(\mathbb{Z} / 2 \mathbb{Z})^{8}$. Degenerations of codimension $1: \mathbb{A}_{1}$ with $A_{S} \cong(\mathbb{Z} / 2 \mathbb{Z})^{9}\left(H_{1,1}\right.$ with the orbit of $\alpha_{2,3}, \alpha_{4,3}, \alpha_{2,4}, \alpha_{4,4}, \alpha_{2,5}$, $\alpha_{4,5}, \alpha_{2,6}$ or $\alpha_{4,6} ; H_{1,2}$ with the orbit of $\alpha_{1,3}, \alpha_{2,3}, \alpha_{3,3}, \alpha_{4,3}, \alpha_{1,4}, \alpha_{2,4}, \alpha_{3,4}$ or $\left.\alpha_{4,4}\right) ; 2 \mathbb{A}_{1}$ with $A_{S} \cong \mathbb{Z} / 4 \mathbb{Z} \times(\mathbb{Z} / 2 \mathbb{Z})^{6}\left(H_{1,1}\right.$ with the orbit of $\alpha_{1,1}, \alpha_{2,1}, \alpha_{3,1}, \alpha_{4,1}, \alpha_{1,3}, \alpha_{1,4}, \alpha_{1,5}$ or $\alpha_{1,6} ; H_{1,2}$ with the orbit of $\alpha_{1,1}, \alpha_{2,1}, \alpha_{3,1}, \alpha_{4,1}, \alpha_{1,5}, \alpha_{2,5}, \alpha_{3,5}$ or $\left.\alpha_{4,5}\right)$.

Table 18. All possible markings by $N_{18}=N\left(4 A_{5} \oplus D_{4}\right)$ of degenerations of codimension one of non-trivial finite symplectic automorphism groups of Kählerian K3 surfaces:

$\mathbf{n}=\mathbf{5 4}, H \cong T_{48}(|H|=48, i=29): \mathrm{rk} N_{H}=19$. No degenerations.

$\mathbf{n}=\mathbf{2 6}, H \cong S D_{16}(|H|=16, i=8): \operatorname{rk} N_{H}=18$ and $\left(N_{H}\right)^{*} / N_{H} \cong(\mathbb{Z} / 8 \mathbb{Z})^{2} \times \mathbb{Z} / 4 \mathbb{Z} \times$ $\mathbb{Z} / 2 \mathbb{Z}$. Degenerations: $8 \mathbb{A}_{1}$ with $A_{S} \cong \mathbb{Z} / 16 \mathbb{Z} \times \mathbb{Z} / 4 \mathbb{Z} \times \mathbb{Z} / 2 \mathbb{Z}\left(H_{26,1}\right.$ with the orbit of $\alpha_{1,1}$ or $\left.\alpha_{2,1}\right)$.

$\mathbf{n}=\mathbf{1 8}, H \cong D_{12}(|H|=12, i=4): \operatorname{rk} N_{H}=16$ and $\left(N_{H}\right)^{*} / N_{H} \cong(\mathbb{Z} / 6 \mathbb{Z})^{4}$. Degenerations of codimension $1: \mathbb{A}_{1}$ with $A_{S} \cong(\mathbb{Z} / 6 \mathbb{Z})^{4} \times \mathbb{Z} / 2 \mathbb{Z}\left(H_{18,1}\right.$ with the orbit of $\alpha_{3,1}$ or $\left.\alpha_{2,5}\right)$; 
$2 \mathbb{A}_{1}$ with $A_{S} \cong \mathbb{Z} / 12 \mathbb{Z} \times(\mathbb{Z} / 6 \mathbb{Z})^{2} \times \mathbb{Z} / 3 \mathbb{Z}\left(H_{18,1}\right.$ with the orbit of $\alpha_{1,1}$ or $\left.\alpha_{2,1}\right) ; 3 \mathbb{A}_{1}$ with $A_{S} \cong(\mathbb{Z} / 6 \mathbb{Z})^{3} \times(\mathbb{Z} / 2 \mathbb{Z})^{2}\left(H_{18,1}\right.$ with the orbit of $\alpha_{3,2}$ or $\left.\alpha_{1,5}\right) ; 6 \mathbb{A}_{1}$ with $A_{S} \cong \mathbb{Z} / 12 \mathbb{Z} \times(\mathbb{Z} / 6 \mathbb{Z})^{2}$ $\left(H_{18,1}\right.$ with the orbit of $\alpha_{1,2}$ or $\left.\alpha_{2,2}\right)$.

$\mathbf{n}=\mathbf{1 2}, H \cong Q_{8}(|H|=8, i=4): \operatorname{rk} N_{H}=17,\left(N_{H}\right)^{*} / N_{H} \cong(\mathbb{Z} / 8 \mathbb{Z})^{2} \times(\mathbb{Z} / 2 \mathbb{Z})^{3}$. Degenerations of codimension 1: $8 \mathbb{A}_{1}$ with $A_{S} \cong \mathbb{Z} / 16 \mathbb{Z} \times(\mathbb{Z} / 2 \mathbb{Z})^{3}\left(H_{12,1}\right.$ with the orbit of $\alpha_{1,1}$ or $\left.\alpha_{2,1}\right)$.

$\mathbf{n}=\mathbf{1 0}, H \cong D_{8}(|H|=8, i=3): \operatorname{rk} N_{H}=15$ and $\left(N_{H}\right)^{*} / N_{H} \cong(\mathbb{Z} / 4 \mathbb{Z})^{5}$. Degenerations of codimension 1: $\mathbb{A}_{1}$ with $A_{S} \cong(\mathbb{Z} / 4 \mathbb{Z})^{5} \times \mathbb{Z} / 2 \mathbb{Z}\left(H_{10,1}\right.$ with the orbit of $\alpha_{2,5}$ or $\left.\alpha_{4,5}\right)$; $2 \mathbb{A}_{1}$ with $A_{S} \cong(\mathbb{Z} / 4 \mathbb{Z})^{4} \times(\mathbb{Z} / 2 \mathbb{Z})^{2}\left(H_{10,1}\right.$ with the orbit of $\alpha_{3,1}, \alpha_{3,3}$ or $\left.\alpha_{1,5}\right) ; 4 \mathbb{A}_{1}$ with $A_{S} \cong \mathbb{Z} / 8 \mathbb{Z} \times(\mathbb{Z} / 4 \mathbb{Z})^{3}\left(H_{10,1}\right.$ with the orbit of $\alpha_{1,1}, \alpha_{2,1}, \alpha_{1,3}$ or $\left.\alpha_{2,3}\right)$.

$\mathbf{n}=6, H \cong D_{6}(|H|=6, i=1): \operatorname{rk} N_{H}=14$ and $\left(N_{H}\right)^{*} / N_{H} \cong(\mathbb{Z} / 6 \mathbb{Z})^{2} \times(\mathbb{Z} / 3 \mathbb{Z})^{3}$. Degenerations of codimension 1 : $\mathbb{A}_{1}$ with $A_{S} \cong(\mathbb{Z} / 6 \mathbb{Z})^{3} \times(\mathbb{Z} / 3 \mathbb{Z})^{2}\left(H_{6,1}\right.$ with the orbit of $\alpha_{1,1}, \alpha_{2,1}, \alpha_{3,1}, \alpha_{4,1}, \alpha_{5,1}$ or $\alpha_{2,5} ; H_{6,2}$ with the orbit of $\alpha_{3,1}$ or $\left.\alpha_{2,5}\right) ; 2 \mathbb{A}_{1}$ with $A_{S} \cong$ $\mathbb{Z} / 12 \mathbb{Z} \times(\mathbb{Z} / 3 \mathbb{Z})^{4}\left(H_{6,2}\right.$ with the orbit of $\alpha_{1,1}$ or $\left.\alpha_{2,1}\right) ; 3 \mathbb{A}_{1}$ with $A_{S} \cong(\mathbb{Z} / 6 \mathbb{Z})^{3} \times \mathbb{Z} / 3 \mathbb{Z}\left(H_{6,1}\right.$ with the orbit of $\alpha_{3,2}$ or $\alpha_{1,5} ; H_{6,2}$ with the orbit of $\alpha_{1,2}, \alpha_{2,2}, \alpha_{3,2}, \alpha_{4,2}, \alpha_{5,2}$ or $\left.\alpha_{1,5}\right) ; 6 \mathbb{A}_{1}$ with $A_{S} \cong \mathbb{Z} / 12 \mathbb{Z} \times(\mathbb{Z} / 3 \mathbb{Z})^{3}\left(H_{6,1}\right.$ with the orbit of $\alpha_{1,2}$ or $\left.\alpha_{2,2}\right)$.

$\mathbf{n}=4, H \cong C_{4}(|H|=4, i=1): \operatorname{rk} N_{H}=14$ and $\left(N_{H}\right)^{*} / N_{H} \cong(\mathbb{Z} / 4 \mathbb{Z})^{4} \times(\mathbb{Z} / 2 \mathbb{Z})^{2}$. Degenerations of codimension 1: $\mathbb{A}_{1}$ with $A_{S} \cong(\mathbb{Z} / 4 \mathbb{Z})^{4} \times(\mathbb{Z} / 2 \mathbb{Z})^{3}\left(H_{4,1}\right.$ with the orbit of $\alpha_{1,5}, \alpha_{2,5}, \alpha_{3,5}$ or $\left.\alpha_{4,5}\right) ; 2 \mathbb{A}_{1}$ with $A_{S} \cong(\mathbb{Z} / 4 \mathbb{Z})^{5}\left(H_{4,1}\right.$ with the orbit of $\alpha_{3,1}$ or $\left.\alpha_{3,3}\right) ; 4 \mathbb{A}_{1}$ with $A_{S} \cong \mathbb{Z} / 8 \mathbb{Z} \times(\mathbb{Z} / 4 \mathbb{Z})^{2} \times(\mathbb{Z} / 2 \mathbb{Z})^{2}\left(H_{4,1}\right.$ with the orbit of $\alpha_{1,1}, \alpha_{2,1}, \alpha_{1,3}$ or $\left.\alpha_{2,3}\right)$.

$\mathbf{n}=\mathbf{3}, H \cong C_{2}^{2}(|H|=4, i=2): \operatorname{rk} N_{H}=12$ and $\left(N_{H}\right)^{*} / N_{H} \cong(\mathbb{Z} / 4 \mathbb{Z})^{2} \times(\mathbb{Z} / 2 \mathbb{Z})^{6}$. Degenerations of codimension 1: $\mathbb{A}_{1}$ with $A_{S} \cong(\mathbb{Z} / 4 \mathbb{Z})^{2} \times(\mathbb{Z} / 2 \mathbb{Z})^{7}\left(H_{3,1}\right.$ with the orbit of $\alpha_{3,1}, \alpha_{3,2}, \alpha_{2,5}$ or $\left.\alpha_{4,5}\right) ; 2 \mathbb{A}_{1}$ with $A_{S} \cong(\mathbb{Z} / 4 \mathbb{Z})^{3} \times(\mathbb{Z} / 2 \mathbb{Z})^{4}\left(H_{3,1}\right.$ with the orbit of $\alpha_{1,1}, \alpha_{2,1}$, $\alpha_{1,2}, \alpha_{2,2}, \alpha_{3,3}$ or $\left.\alpha_{1,5}\right) ; 4 \mathbb{A}_{1}$ with $A_{S} \cong \mathbb{Z} / 8 \mathbb{Z} \times(\mathbb{Z} / 2 \mathbb{Z})^{6}\left(H_{3,1}\right.$ with the orbit of $\alpha_{1,3}$ or $\left.\alpha_{1,4}\right)$.

$\mathbf{n}=\mathbf{2}, H \cong C_{3}(|H|=3, i=1): \operatorname{rk} N_{H}=12$ and $\left(N_{H}\right)^{*} / N_{H} \cong(\mathbb{Z} / 3 \mathbb{Z})^{6}$. Degenerations of codimension 1 : $\mathbb{A}_{1}$ with $A_{S} \cong \mathbb{Z} / 6 \mathbb{Z} \times(\mathbb{Z} / 3 \mathbb{Z})^{5}\left(H_{2,1}\right.$ with the orbit of $\alpha_{1,1}, \alpha_{2,1}, \alpha_{3,1}, \alpha_{4,1}$, $\alpha_{5,1}$ or $\left.\alpha_{2,5}\right) ; 3 \mathbb{A}_{1}$ with $A_{S} \cong \mathbb{Z} / 6 \mathbb{Z} \times(\mathbb{Z} / 3 \mathbb{Z})^{4}\left(H_{2,1}\right.$ with the orbit of $\alpha_{1,2}, \alpha_{2,2}, \alpha_{3,2}, \alpha_{4,2}, \alpha_{5,2}$ or $\left.\alpha_{1,5}\right)$.

$\mathbf{n}=\mathbf{1}, H \cong C_{2}(|H|=2, i=1): \operatorname{rk} N_{H}=8$ and $\left(N_{H}\right)^{*} / N_{H} \cong(\mathbb{Z} / 2 \mathbb{Z})^{8}$. Degenerations of codimension 1: $\mathbb{A}_{1}$ with $A_{S} \cong(\mathbb{Z} / 2 \mathbb{Z})^{9}\left(H_{1,1}\right.$ with the orbit of $\alpha_{1,1}, \alpha_{2,1}, \alpha_{3,1}, \alpha_{4,1}, \alpha_{5,1}$, $\alpha_{3,2}, \alpha_{2,5}$ or $\alpha_{4,5} ; H_{1,2}$ with the orbit of $\alpha_{3,1}, \alpha_{3,2}, \alpha_{3,3}, \alpha_{3,4}, \alpha_{1,5}, \alpha_{2,5}, \alpha_{3,5}$ or $\left.\alpha_{4,5}\right) ; 2 \mathbb{A}_{1}$ with $A_{S} \cong \mathbb{Z} / 4 \mathbb{Z} \times(\mathbb{Z} / 2 \mathbb{Z})^{6}\left(H_{1,1}\right.$ with the orbit of $\alpha_{1,2}, \alpha_{2,2}, \alpha_{1,3}, \alpha_{2,3}, \alpha_{3,3}, \alpha_{4,3}, \alpha_{5,3}$ or $\alpha_{1,5} ; H_{1,2}$ with the orbit of $\alpha_{1,1}, \alpha_{2,1}, \alpha_{1,2}, \alpha_{2,2}, \alpha_{1,3}, \alpha_{2,3}, \alpha_{1,4}$ or $\left.\alpha_{2,4}\right)$. 
Table 17. All possible markings by $N_{17}=N\left(4 A_{6}\right)$ of degenerations of codimension one of non-trivial finite symplectic automorphism groups of Kählerian K3 surfaces:

$\mathbf{n}=\mathbf{2}, H \cong C_{3}(|H|=3, i=1): \operatorname{rk} N_{H}=12$ and $\left(N_{H}\right)^{*} / N_{H} \cong(\mathbb{Z} / 3 \mathbb{Z})^{6}$. Degenerations of codimension 1: $\mathbb{A}_{1}$ with $A_{S} \cong \mathbb{Z} / 6 \mathbb{Z} \times(\mathbb{Z} / 3 \mathbb{Z})^{5}\left(H_{2,1}\right.$ with the orbit of $\alpha_{1,1}, \alpha_{2,1}, \alpha_{3,1}, \alpha_{4,1}$, $\alpha_{5,1}$ or $\left.\alpha_{6,1}\right) ; 3 \mathbb{A}_{1}$ with $A_{S} \cong \mathbb{Z} / 6 \mathbb{Z} \times(\mathbb{Z} / 3 \mathbb{Z})^{4}\left(H_{2,1}\right.$ with the orbit of $\alpha_{1,2}, \alpha_{2,2}, \alpha_{3,2}, \alpha_{4,2}, \alpha_{5,2}$ or $\left.\alpha_{6,2}\right)$.

Table 16. All possible markings by $N_{16}=N\left(2 A_{7} \oplus 2 D_{5}\right)$ of degenerations of codimension one of non-trivial finite symplectic automorphism groups of Kählerian K3 surfaces:

$\mathbf{n}=\mathbf{3}, H \cong C_{2}^{2}(|H|=4, i=2): \operatorname{rk} N_{H}=12$ and $\left(N_{H}\right)^{*} / N_{H} \cong(\mathbb{Z} / 4 \mathbb{Z})^{2} \times(\mathbb{Z} / 2 \mathbb{Z})^{6}$. Degenerations of codimension $1: \mathbb{A}_{1}$ with $A_{S} \cong(\mathbb{Z} / 4 \mathbb{Z})^{2} \times(\mathbb{Z} / 2 \mathbb{Z})^{7}\left(H_{3,1}\right.$ with the orbit of $\alpha_{4,1}$ or $\alpha_{4,2} ; H_{3,2}$ with the orbit of $\alpha_{1,3}, \alpha_{2,3}, \alpha_{3,3}, \alpha_{1,4}, \alpha_{2,4}$ or $\left.\alpha_{3,4}\right) ; 2 \mathbb{A}_{1}$ with $A_{S} \cong$ $(\mathbb{Z} / 4 \mathbb{Z})^{3} \times(\mathbb{Z} / 2 \mathbb{Z})^{4}\left(H_{3,1}\right.$ with the orbit of $\alpha_{1,1}, \alpha_{2,1}, \alpha_{3,1}, \alpha_{1,2}, \alpha_{2,2}, \alpha_{3,2}, \alpha_{1,3}, \alpha_{2,3}$ or $\alpha_{3,3}$; $H_{3,2}$ with the orbit of $\alpha_{4,1}, \alpha_{4,3}$ or $\left.\alpha_{4,4}\right) ; 4 \mathbb{A}_{1}$ with $A_{S} \cong \mathbb{Z} / 8 \mathbb{Z} \times(\mathbb{Z} / 2 \mathbb{Z})^{6}\left(H_{3,1}\right.$ with the orbit of $\alpha_{4,3} ; H_{3,2}$ with the orbit of $\alpha_{1,1}, \alpha_{2,1}$ or $\left.\alpha_{3,1}\right)$.

$\mathbf{n}=\mathbf{1}, H \cong C_{2}(|H|=2, i=1): \operatorname{rk} N_{H}=8$ and $\left(N_{H}\right)^{*} / N_{H} \cong(\mathbb{Z} / 2 \mathbb{Z})^{8}$. Degenerations of codimension $1: \mathbb{A}_{1}$ with $A_{S} \cong(\mathbb{Z} / 2 \mathbb{Z})^{9}\left(H_{1,1}\right.$ with the orbit of $\alpha_{1,1}, \alpha_{2,1}, \alpha_{3,1}, \alpha_{4,1}, \alpha_{5,1}, \alpha_{6,1}$, $\alpha_{7,1}$ or $\alpha_{4,2} ; H_{1,2}$ with the orbit of $\alpha_{4,1}, \alpha_{4,2}, \alpha_{1,3}, \alpha_{2,3}, \alpha_{3,3}, \alpha_{1,4}, \alpha_{2,4}$ or $\alpha_{3,4} ; H_{1,3}$ with the orbit of $\alpha_{1,3}, \alpha_{2,3}, \alpha_{3,3}, \alpha_{4,3}, \alpha_{5,3}, \alpha_{1,4}, \alpha_{2,4}$ or $\left.\alpha_{3,4}\right) ; 2 \mathbb{A}_{1}$ with $A_{S} \cong \mathbb{Z} / 4 \mathbb{Z} \times(\mathbb{Z} / 2 \mathbb{Z})^{6}\left(H_{1,1}\right.$ with the orbit of $\alpha_{1,2}, \alpha_{2,2}, \alpha_{3,2}, \alpha_{1,3}, \alpha_{2,3}, \alpha_{3,3}, \alpha_{4,3}$ or $\alpha_{5,3} ; H_{1,2}$ with the orbit of $\alpha_{1,1}, \alpha_{2,1}$, $\alpha_{3,1}, \alpha_{1,2}, \alpha_{2,2}, \alpha_{3,2}, \alpha_{4,3}$ or $\alpha_{4,4} ; H_{1,3}$ with the orbit of $\alpha_{1,1}, \alpha_{2,1}, \alpha_{3,1}, \alpha_{4,1}, \alpha_{5,1}, \alpha_{6,1}, \alpha_{7,1}$ or $\left.\alpha_{4,4}\right)$.

Table 15. All possible markings by $N_{15}=N\left(3 A_{8}\right)$ of degenerations of codimension one of non-trivial finite symplectic automorphism groups of Kählerian K3 surfaces:

$\mathbf{n}=\mathbf{1}, H \cong C_{2}(|H|=2, i=1): \operatorname{rk} N_{H}=8$ and $\left(N_{H}\right)^{*} / N_{H} \cong(\mathbb{Z} / 2 \mathbb{Z})^{8}$. Degenerations of codimension 1: $\mathbb{A}_{1}$ with $A_{S} \cong(\mathbb{Z} / 2 \mathbb{Z})^{9}\left(H_{1,1}\right.$ with the orbit of $\alpha_{1,1}, \alpha_{2,1}, \alpha_{3,1}, \alpha_{4,1}, \alpha_{5,1}, \alpha_{6,1}$, $\alpha_{7,1}$ or $\left.\alpha_{8,1}\right) ; 2 \mathbb{A}_{1}$ with $A_{S} \cong \mathbb{Z} / 4 \mathbb{Z} \times(\mathbb{Z} / 2 \mathbb{Z})^{6}\left(H_{1,1}\right.$ with the orbit of $\alpha_{1,2}, \alpha_{2,2}, \alpha_{3,2}, \alpha_{4,2}$, $\alpha_{5,2}, \alpha_{6,2}, \alpha_{7,2}$ or $\left.\alpha_{8,2}\right)$. 
Table 14. All possible markings by $N_{14}=N\left(4 D_{6}\right)$ of degenerations of codimension one of non-trivial finite symplectic automorphism groups of Kählerian K3 surfaces:

$\mathbf{n}=6, H \cong D_{6}(|H|=6, i=1): \operatorname{rk} N_{H}=14$ and $\left(N_{H}\right)^{*} / N_{H} \cong(\mathbb{Z} / 6 \mathbb{Z})^{2} \times(\mathbb{Z} / 3 \mathbb{Z})^{3}$. Degenerations of codimension 1 : $\mathbb{A}_{1}$ with $A_{S} \cong(\mathbb{Z} / 6 \mathbb{Z})^{3} \times(\mathbb{Z} / 3 \mathbb{Z})^{2}\left(H_{6,1}\right.$ with the orbit of $\alpha_{1,1}, \alpha_{2,1}, \alpha_{3,1}$ or $\left.\alpha_{4,1}\right) ; 2 \mathbb{A}_{1}$ with $A_{S} \cong \mathbb{Z} / 12 \mathbb{Z} \times(\mathbb{Z} / 3 \mathbb{Z})^{4}\left(H_{6,1}\right.$ with the orbit of $\left.\alpha_{5,1}\right)$; $3 \mathbb{A}_{1}$ with $A_{S} \cong(\mathbb{Z} / 6 \mathbb{Z})^{3} \times \mathbb{Z} / 3 \mathbb{Z}\left(H_{6,1}\right.$ with the orbit of $\alpha_{1,2}, \alpha_{2,2}, \alpha_{3,2}$ or $\left.\alpha_{4,2}\right) ; 6 \mathbb{A}_{1}$ with $A_{S} \cong \mathbb{Z} / 12 \mathbb{Z} \times(\mathbb{Z} / 3 \mathbb{Z})^{3}\left(H_{6,1}\right.$ with the orbit of $\left.\alpha_{5,2}\right)$.

$\mathbf{n}=\mathbf{2}, H \cong C_{3}(|H|=3, i=1): \operatorname{rk} N_{H}=12$ and $\left(N_{H}\right)^{*} / N_{H} \cong(\mathbb{Z} / 3 \mathbb{Z})^{6}$. Degenerations of codimension 1: $\mathbb{A}_{1}$ with $A_{S} \cong \mathbb{Z} / 6 \mathbb{Z} \times(\mathbb{Z} / 3 \mathbb{Z})^{5}\left(H_{2,1}\right.$ with the orbit of $\alpha_{1,1}, \alpha_{2,1}, \alpha_{3,1}, \alpha_{4,1}$, $\alpha_{5,1}$ or $\left.\alpha_{6,1}\right) ; 3 \mathbb{A}_{1}$ with $A_{S} \cong \mathbb{Z} / 6 \mathbb{Z} \times(\mathbb{Z} / 3 \mathbb{Z})^{4}\left(H_{2,1}\right.$ with the orbit of $\alpha_{1,2}, \alpha_{2,2}, \alpha_{3,2}, \alpha_{4,2}, \alpha_{5,2}$ or $\left.\alpha_{6,2}\right)$.

$\mathbf{n}=\mathbf{1}, H \cong C_{2}(|H|=2, i=1): \operatorname{rk} N_{H}=8$ and $\left(N_{H}\right)^{*} / N_{H} \cong(\mathbb{Z} / 2 \mathbb{Z})^{8}$. Degenerations of codimension 1: $\mathbb{A}_{1}$ with $A_{S} \cong(\mathbb{Z} / 2 \mathbb{Z})^{9}\left(H_{1,1}\right.$ with the orbit of $\alpha_{1,1}, \alpha_{2,1}, \alpha_{3,1}, \alpha_{4,1}, \alpha_{1,2}, \alpha_{2,2}$, $\alpha_{3,2}$ or $\left.\alpha_{4,2}\right) ; 2 \mathbb{A}_{1}$ with $A_{S} \cong \mathbb{Z} / 4 \mathbb{Z} \times(\mathbb{Z} / 2 \mathbb{Z})^{6}\left(H_{1,1}\right.$ with the orbit of $\alpha_{5,1}, \alpha_{5,2}, \alpha_{1,3}, \alpha_{2,3}$, $\alpha_{3,3}, \alpha_{4,3}, \alpha_{5,3}$ or $\left.\alpha_{6,3}\right)$.

Table 13. All possible markings by $N_{13}=N\left(2 A_{9} \oplus D_{6}\right)$ of degenerations of codimension one of non-trivial finite symplectic automorphism groups of Kählerian K3 surfaces:

$\mathbf{n}=4, H \cong C_{4}(|H|=4, i=1): \operatorname{rk} N_{H}=14$ and $\left(N_{H}\right)^{*} / N_{H} \cong(\mathbb{Z} / 4 \mathbb{Z})^{4} \times(\mathbb{Z} / 2 \mathbb{Z})^{2}$. Degenerations of codimension 1 : $\mathbb{A}_{1}$ with $A_{S} \cong(\mathbb{Z} / 4 \mathbb{Z})^{4} \times(\mathbb{Z} / 2 \mathbb{Z})^{3}\left(H_{4,1}\right.$ with the orbit of $\alpha_{1,3}, \alpha_{2,3}, \alpha_{3,3}$ or $\left.\alpha_{4,3}\right) ; 2 \mathbb{A}_{1}$ with $A_{S} \cong(\mathbb{Z} / 4 \mathbb{Z})^{5}\left(H_{4,1}\right.$ with the orbit of $\alpha_{2,1}$ or $\left.\alpha_{5,3}\right) ; 4 \mathbb{A}_{1}$ with $A_{S} \cong \mathbb{Z} / 8 \mathbb{Z} \times(\mathbb{Z} / 4 \mathbb{Z})^{2} \times(\mathbb{Z} / 2 \mathbb{Z})^{2}\left(H_{4,1}\right.$ with the orbit of $\alpha_{1,1}, \alpha_{2,1}, \alpha_{3,1}$ or $\left.\alpha_{4,1}\right)$.

$\mathbf{n}=\mathbf{1}, H \cong C_{2}(|H|=2, i=1): \operatorname{rk} N_{H}=8$ and $\left(N_{H}\right)^{*} / N_{H} \cong(\mathbb{Z} / 2 \mathbb{Z})^{8}$. Degenerations of codimension $1: \mathbb{A}_{1}$ with $A_{S} \cong(\mathbb{Z} / 2 \mathbb{Z})^{9}\left(H_{1,1}\right.$ with the orbit of $\alpha_{5,1}, \alpha_{5,2}, \alpha_{1,3}, \alpha_{2,3}, \alpha_{3,3}, \alpha_{4,3}$, $\alpha_{5,3}$ or $\left.\alpha_{6,3}\right) ; 2 \mathbb{A}_{1}$ with $A_{S} \cong \mathbb{Z} / 4 \mathbb{Z} \times(\mathbb{Z} / 2 \mathbb{Z})^{6}\left(H_{1,1}\right.$ with the orbit of $\alpha_{1,1}, \alpha_{2,1}, \alpha_{3,1}, \alpha_{4,1}$, $\alpha_{1,2}, \alpha_{2,2}, \alpha_{3,2}$ or $\left.\alpha_{4,2}\right)$.

Table 12. All possible markings by $N_{12}=N\left(4 E_{6}\right)$ of degenerations of codimension one of non-trivial finite symplectic automorphism groups of Kählerian K3 surfaces:

$\mathbf{n}=\mathbf{1 8}, H \cong D_{12}(|H|=12, i=4): \operatorname{rk} N_{H}=16$ and $\left(N_{H}\right)^{*} / N_{H} \cong(\mathbb{Z} / 6 \mathbb{Z})^{4}$. Degenerations of codimension 1 : $\mathbb{A}_{1}$ with $A_{S} \cong(\mathbb{Z} / 6 \mathbb{Z})^{4} \times \mathbb{Z} / 2 \mathbb{Z}\left(H_{18,1}\right.$ with the orbit of $\alpha_{2,1}$ or $\left.\alpha_{4,1}\right)$; 
$2 \mathbb{A}_{1}$ with $A_{S} \cong \mathbb{Z} / 12 \mathbb{Z} \times(\mathbb{Z} / 6 \mathbb{Z})^{2} \times \mathbb{Z} / 3 \mathbb{Z}\left(H_{18,1}\right.$ with the orbit of $\alpha_{1,1}$ or $\left.\alpha_{3,1}\right) ; 3 \mathbb{A}_{1}$ with $A_{S} \cong(\mathbb{Z} / 6 \mathbb{Z})^{3} \times(\mathbb{Z} / 2 \mathbb{Z})^{2}\left(H_{18,1}\right.$ with the orbit of $\alpha_{2,2}$ or $\left.\alpha_{4,2}\right) ; 6 \mathbb{A}_{1}$ with $A_{S} \cong \mathbb{Z} / 12 \mathbb{Z} \times(\mathbb{Z} / 6 \mathbb{Z})^{2}$ $\left(H_{18,1}\right.$ with the orbit of $\alpha_{1,2}$ or $\left.\alpha_{3,2}\right)$.

$\mathbf{n}=\mathbf{6}, H \cong D_{6}(|H|=6, i=1): \operatorname{rk} N_{H}=14$ and $\left(N_{H}\right)^{*} / N_{H} \cong(\mathbb{Z} / 6 \mathbb{Z})^{2} \times(\mathbb{Z} / 3 \mathbb{Z})^{3}$. Degenerations of codimension 1: $\mathbb{A}_{1}$ with $A_{S} \cong(\mathbb{Z} / 6 \mathbb{Z})^{3} \times(\mathbb{Z} / 3 \mathbb{Z})^{2}\left(H_{6,1}\right.$ with the orbit of $\alpha_{1,1}, \alpha_{2,1}, \alpha_{3,1}, \alpha_{4,1}, \alpha_{5,1}$ or $\alpha_{6,1} ; H_{6,2}$ with the orbit of $\alpha_{2,1}$ or $\left.\alpha_{4,1}\right) ; 2 \mathbb{A}_{1}$ with $A_{S} \cong$ $\mathbb{Z} / 12 \mathbb{Z} \times(\mathbb{Z} / 3 \mathbb{Z})^{4}\left(H_{6,2}\right.$ with the orbit of $\alpha_{1,1}$ or $\left.\alpha_{3,1}\right) ; 3 \mathbb{A}_{1}$ with $A_{S} \cong(\mathbb{Z} / 6 \mathbb{Z})^{3} \times \mathbb{Z} / 3 \mathbb{Z}\left(H_{6,1}\right.$ with the orbit of $\alpha_{2,2}$ or $\alpha_{4,2} ; H_{6,2}$ with the orbit of $\alpha_{1,2}, \alpha_{2,2}, \alpha_{3,2}, \alpha_{4,2}, \alpha_{5,2}$ or $\left.\alpha_{6,2}\right) ; 6 \mathbb{A}_{1}$ with $A_{S} \cong \mathbb{Z} / 12 \mathbb{Z} \times(\mathbb{Z} / 3 \mathbb{Z})^{3}\left(H_{6,1}\right.$ with the orbit of $\alpha_{1,2}$ or $\left.\alpha_{3,2}\right)$.

$$
\mathbf{n}=3, H \cong C_{2}^{2}(|H|=4, i=2): \operatorname{rk} N_{H}=12 \text { and }\left(N_{H}\right)^{*} / N_{H} \cong(\mathbb{Z} / 4 \mathbb{Z})^{2} \times(\mathbb{Z} / 2 \mathbb{Z})^{6} .
$$
Degenerations of codimension 1: $\mathbb{A}_{1}$ with $A_{S} \cong(\mathbb{Z} / 4 \mathbb{Z})^{2} \times(\mathbb{Z} / 2 \mathbb{Z})^{7}\left(H_{3,1}\right.$ with the orbit of $\alpha_{2,1}, \alpha_{4,1}, \alpha_{2,2}$ or $\left.\alpha_{4,2}\right) ; 2 \mathbb{A}_{1}$ with $A_{S} \cong(\mathbb{Z} / 4 \mathbb{Z})^{3} \times(\mathbb{Z} / 2 \mathbb{Z})^{4}\left(H_{3,1}\right.$ with the orbit of $\alpha_{1,1}, \alpha_{3,1}$, $\alpha_{1,2}, \alpha_{3,2}, \alpha_{2,3}$ or $\left.\alpha_{4,3}\right) ; 4 \mathbb{A}_{1}$ with $A_{S} \cong \mathbb{Z} / 8 \mathbb{Z} \times(\mathbb{Z} / 2 \mathbb{Z})^{6}\left(H_{3,1}\right.$ with the orbit of $\alpha_{1,3}$ or $\left.\alpha_{3,3}\right)$.

$\mathbf{n}=\mathbf{2}, H \cong C_{3}(|H|=3, i=1): \operatorname{rk} N_{H}=12$ and $\left(N_{H}\right)^{*} / N_{H} \cong(\mathbb{Z} / 3 \mathbb{Z})^{6}$. Degenerations of codimension 1 : $\mathbb{A}_{1}$ with $A_{S} \cong \mathbb{Z} / 6 \mathbb{Z} \times(\mathbb{Z} / 3 \mathbb{Z})^{5}\left(H_{2,1}\right.$ with the orbit of $\alpha_{1,1}, \alpha_{2,1}, \alpha_{3,1}, \alpha_{4,1}$, $\alpha_{5,1}$ or $\left.\alpha_{6,1}\right) ; 3 \mathbb{A}_{1}$ with $A_{S} \cong \mathbb{Z} / 6 \mathbb{Z} \times(\mathbb{Z} / 3 \mathbb{Z})^{4}\left(H_{2,1}\right.$ with the orbit of $\alpha_{1,2}, \alpha_{2,2}, \alpha_{3,2}, \alpha_{4,2}, \alpha_{5,2}$ or $\left.\alpha_{6,2}\right)$.

$\mathbf{n}=\mathbf{1}, H \cong C_{2}(|H|=2, i=1): \operatorname{rk} N_{H}=8$ and $\left(N_{H}\right)^{*} / N_{H} \cong(\mathbb{Z} / 2 \mathbb{Z})^{8}$. Degenerations of codimension 1: $\mathbb{A}_{1}$ with $A_{S} \cong(\mathbb{Z} / 2 \mathbb{Z})^{9}\left(H_{1,1}\right.$ with the orbit of $\alpha_{2,1}, \alpha_{4,1}, \alpha_{2,2}, \alpha_{4,2}, \alpha_{2,3}$, $\alpha_{4,3}, \alpha_{2,4}$ or $\alpha_{4,4} ; H_{1,2}$ with the orbit of $\alpha_{2,1}, \alpha_{4,1}, \alpha_{1,2}, \alpha_{2,2}, \alpha_{3,2}, \alpha_{4,2}, \alpha_{5,2}$ or $\left.\alpha_{6,2}\right) ; 2 \mathbb{A}_{1}$ with $A_{S} \cong \mathbb{Z} / 4 \mathbb{Z} \times(\mathbb{Z} / 2 \mathbb{Z})^{6}\left(H_{1,1}\right.$ with the orbit of $\alpha_{1,1}, \alpha_{1,2}, \alpha_{1,3}, \alpha_{1,4}, \alpha_{3,1}, \alpha_{3,2}, \alpha_{3,3}$ or $\alpha_{3,4} ; H_{1,2}$ with the orbit of $\alpha_{1,1}, \alpha_{1,3}, \alpha_{6,3}, \alpha_{3,1}, \alpha_{2,3}, \alpha_{3,3}, \alpha_{4,3}$ or $\left.\alpha_{5,3}\right)$.

Table 11. All possible markings by $N_{11}=N\left(A_{11} \oplus D_{7} \oplus E_{6}\right)$ of degenerations of codimension one of non-trivial finite symplectic automorphism groups of Kählerian K3 surfaces:

$\mathbf{n}=\mathbf{1}, H \cong C_{2}(|H|=2, i=1): \operatorname{rk} N_{H}=8$ and $\left(N_{H}\right)^{*} / N_{H} \cong(\mathbb{Z} / 2 \mathbb{Z})^{8}$. Degenerations of codimension 1: $\mathbb{A}_{1}$ with $A_{S} \cong(\mathbb{Z} / 2 \mathbb{Z})^{9}\left(H_{1,1}\right.$ with the orbit of $\alpha_{6,1}, \alpha_{1,2}, \alpha_{2,2}, \alpha_{3,2}, \alpha_{4,2}, \alpha_{5,2}$, $\alpha_{2,3}$ or $\left.\alpha_{4,3}\right) ; 2 \mathbb{A}_{1}$ with $A_{S} \cong \mathbb{Z} / 4 \mathbb{Z} \times(\mathbb{Z} / 2 \mathbb{Z})^{6}\left(H_{1,1}\right.$ with the orbit of $\alpha_{1,1}, \alpha_{2,1}, \alpha_{3,1}, \alpha_{4,1}$, $\alpha_{5,1}, \alpha_{6,2}, \alpha_{1,3}$ or $\left.\alpha_{3,3}\right)$.

Table 9. All possible markings by $N_{9}=N\left(3 D_{8}\right)$ of degenerations of codimension one of non-trivial finite symplectic automorphism groups of Kählerian K3 surfaces: 
$\mathbf{n}=\mathbf{1}, H \cong C_{2}(|H|=2, i=1): \operatorname{rk} N_{H}=8$ and $\left(N_{H}\right)^{*} / N_{H} \cong(\mathbb{Z} / 2 \mathbb{Z})^{8}$. Degenerations of codimension 1: $\mathbb{A}_{1}$ with $A_{S} \cong(\mathbb{Z} / 2 \mathbb{Z})^{9}\left(H_{1,1}\right.$ with the orbit of $\alpha_{1,3}, \alpha_{2,3}, \alpha_{3,3}, \alpha_{4,3}, \alpha_{5,3}, \alpha_{6,3}$, $\alpha_{7,3}$ or $\left.\alpha_{8,3}\right) ; 2 \mathbb{A}_{1}$ with $A_{S} \cong \mathbb{Z} / 4 \mathbb{Z} \times(\mathbb{Z} / 2 \mathbb{Z})^{6}\left(H_{1,1}\right.$ with the orbit of $\alpha_{1,1}, \alpha_{2,1}, \alpha_{3,1}, \alpha_{4,1}$, $\alpha_{5,1}, \alpha_{6,1}, \alpha_{7,1}$ or $\left.\alpha_{8,1}\right)$.

Table 8. All possible markings by $N_{8}=N\left(A_{15} \oplus D_{9}\right)$ of degenerations of codimension one of non-trivial finite symplectic automorphism groups of Kählerian K3 surfaces:

$\mathbf{n}=\mathbf{1}, H \cong C_{2}(|H|=2, i=1): \operatorname{rk} N_{H}=8$ and $\left(N_{H}\right)^{*} / N_{H} \cong(\mathbb{Z} / 2 \mathbb{Z})^{8}$. Degenerations of codimension $1: \mathbb{A}_{1}$ with $A_{S} \cong(\mathbb{Z} / 2 \mathbb{Z})^{9}\left(H_{1,1}\right.$ with the orbit of $\alpha_{8,1}, \alpha_{1,2}, \alpha_{2,2}, \alpha_{3,2}, \alpha_{4,2}, \alpha_{5,2}$, $\alpha_{6,2}$ or $\left.\alpha_{7,2}\right) ; 2 \mathbb{A}_{1}$ with $A_{S} \cong \mathbb{Z} / 4 \mathbb{Z} \times(\mathbb{Z} / 2 \mathbb{Z})^{6}\left(H_{1,1}\right.$ with the orbit of $\alpha_{1,1}, \alpha_{2,1}, \alpha_{3,1}, \alpha_{4,1}$, $\alpha_{5,1}, \alpha_{6,1}, \alpha_{7,1}$ or $\left.\alpha_{8,2}\right)$.

Table 7. All possible markings by $N_{7}=N\left(D_{10} \oplus 2 E_{7}\right)$ of degenerations of codimension one of non-trivial finite symplectic automorphism groups of Kählerian K3 surfaces:

$\mathbf{n}=\mathbf{1}, H \cong C_{2}(|H|=2, i=1): \operatorname{rk} N_{H}=8$ and $\left(N_{H}\right)^{*} / N_{H} \cong(\mathbb{Z} / 2 \mathbb{Z})^{8}$. Degenerations of codimension 1 : $\mathbb{A}_{1}$ with $A_{S} \cong(\mathbb{Z} / 2 \mathbb{Z})^{9}\left(H_{1,1}\right.$ with the orbit of $\alpha_{1,1}, \alpha_{2,1}, \alpha_{3,1}, \alpha_{4,1}, \alpha_{5,1}, \alpha_{6,1}$, $\alpha_{7,1}$ or $\left.\alpha_{8,1}\right) ; 2 \mathbb{A}_{1}$ with $A_{S} \cong \mathbb{Z} / 4 \mathbb{Z} \times(\mathbb{Z} / 2 \mathbb{Z})^{6}\left(H_{1,1}\right.$ with the orbit of $\alpha_{9,1}, \alpha_{1,2}, \alpha_{2,2}, \alpha_{3,2}$, $\alpha_{4,2}, \alpha_{5,2}, \alpha_{6,2}$ or $\left.\alpha_{7,2}\right)$.

Table 6. All possible markings by $N_{6}=N\left(A_{17} \oplus E_{7}\right)$ of degenerations of codimension one of non-trivial finite symplectic automorphism groups of Kählerian K3 surfaces:

$\mathbf{n}=\mathbf{1}, H \cong C_{2}(|H|=2, i=1): \operatorname{rk} N_{H}=8$ and $\left(N_{H}\right)^{*} / N_{H} \cong(\mathbb{Z} / 2 \mathbb{Z})^{8}$. Degenerations of codimension 1: $\mathbb{A}_{1}$ with $A_{S} \cong(\mathbb{Z} / 2 \mathbb{Z})^{9}\left(H_{1,1}\right.$ with the orbit of $\alpha_{9,1}, \alpha_{1,2}, \alpha_{2,2}, \alpha_{3,2}, \alpha_{4,2}, \alpha_{5,2}$, $\alpha_{6,2}$ or $\left.\alpha_{7,2}\right) ; 2 \mathbb{A}_{1}$ with $A_{S} \cong \mathbb{Z} / 4 \mathbb{Z} \times(\mathbb{Z} / 2 \mathbb{Z})^{6}\left(H_{1,1}\right.$ with the orbit of $\alpha_{1,1}, \alpha_{2,1}, \alpha_{3,1}, \alpha_{4,1}$, $\alpha_{5,1}, \alpha_{6,1}, \alpha_{7,1}$ or $\left.\alpha_{8,1}\right)$.

Table 3. All possible markings by $N_{3}=N\left(3 E_{8}\right)$ of degenerations of codimension one of non-trivial finite symplectic automorphism groups of Kählerian K3 surfaces: 
$\mathbf{n}=\mathbf{1}, H \cong C_{2}(|H|=2, i=1): \operatorname{rk} N_{H}=8$ and $\left(N_{H}\right)^{*} / N_{H} \cong(\mathbb{Z} / 2 \mathbb{Z})^{8}$. Degenerations of codimension 1: $\mathbb{A}_{1}$ with $A_{S} \cong(\mathbb{Z} / 2 \mathbb{Z})^{9}\left(H_{1,1}\right.$ with the orbit of $\alpha_{1,3}, \alpha_{2,3}, \alpha_{3,3}, \alpha_{4,3}, \alpha_{5,3}, \alpha_{6,3}$, $\alpha_{7,3}$ or $\left.\alpha_{8,3}\right) ; 2 \mathbb{A}_{1}$ with $A_{S} \cong \mathbb{Z} / 4 \mathbb{Z} \times(\mathbb{Z} / 2 \mathbb{Z})^{6}\left(H_{1,1}\right.$ with the orbit of $\alpha_{1,1}, \alpha_{2,1}, \alpha_{3,1}, \alpha_{4,1}$, $\alpha_{5,1}, \alpha_{6,1}, \alpha_{7,1}$ or $\left.\alpha_{8,1}\right)$. 


\section{Classification of types of degenerations of finite symplectic automorphism groups of Kählerian K3 surfaces}

Using Theorem 2, we obtain the following classification of types of degenerations of codimension 1 of Kählerian K3 surfaces.

Theorem 3. For non-trivial finite symplectic automorphism groups $H$ of Kählerian K3 surfaces $X$, types of degenerations of codimension one $N_{H} \subset S$ (that is $\operatorname{rk} S=\operatorname{rk} N_{H}+1$ and $S<0)$ are the following and only the following which are given in Table GEN below. We give the type of the set of non-singular rational curves of $X$ and the isomorphism class of the discriminant group $A_{S}=S^{*} / S$ of $S=S_{X}$. The description of these degenerations using markings by Niemeier lattices $N_{j}, j=3,6,7,8,9,11-23$, are given in Theorem 2 above and its Tables $j$.

Table GEN. Classification of types of degenerations of codimension one of non-trivial finite symplectic automorphism groups of Kählerian K3 surfaces:

$\mathbf{n}=\mathbf{8 1}, H \cong M_{20}(|H|=960, i=11357):$ rk $N_{H}=19$. No degenerations.

$\mathbf{n}=80, H \cong F_{384}(|H|=384, i=18135):$ rk $N_{H}=19$. No degenerations.

$\mathbf{n}=\mathbf{7 9}, H \cong \mathfrak{A}_{6}(|H|=360, i=118)$ : rk $N_{H}=19$. No degenerations.

$\mathbf{n}=\mathbf{7 8}, H \cong \mathfrak{A}_{4,4}(|H|=288, i=1026):$ rk $N_{H}=19$. No degenerations.

$\mathbf{n}=77, H \cong T_{192}(|H|=192, i=1493):$ rk $N_{H}=19$. No degenerations.

$\mathbf{n}=76, H \cong H_{192}(|H|=192, i=955): \operatorname{rk} N_{H}=19$. No degenerations.

$\mathbf{n}=\mathbf{7 5}, H \cong 4^{2} \mathfrak{A}_{4}(|H|=192, i=1023): \operatorname{rk} N_{H}=18,\left(N_{H}\right)^{*} / N_{H} \cong(\mathbb{Z} / 8 \mathbb{Z})^{2} \times(\mathbb{Z} / 2 \mathbb{Z})^{2}$. Degenerations: $16 \mathbb{A}_{1}$ with $A_{S} \cong(\mathbb{Z} / 8 \mathbb{Z}) \times(\mathbb{Z} / 2 \mathbb{Z})^{2}$.

$\mathbf{n}=\mathbf{7 4}, H \cong L_{2}(7)(|H|=168, i=42)$ : rk $N_{H}=19$. No degenerations.

$\mathbf{n}=73, H \cong 2^{4} D_{10}(|H|=160, i=234)$ : The same as for $n=81$ above.

$\mathbf{n}=\mathbf{7 2}, H \cong \mathfrak{A}_{4}^{2}(|H|=144, i=184)$ : The same as for $n=78$ above.

$\mathbf{n}=\mathbf{7 1}, H \cong F_{128}(|H|=128, i=931)$ : The same as for $n=80$ above.

$\mathbf{n}=\mathbf{7 0}, H \cong \mathfrak{S}_{5}(|H|=120, i=34)$ : rk $N_{H}=19$. No degenerations.

$\mathbf{n}=69, H \cong\left(Q_{8} * Q_{8}\right) \rtimes C_{3}(|H|=96, i=204)$ : The same as for $n=77$ above. 
$\mathbf{n}=68, H \cong 2^{3} D_{12},(|H|=96, i=195)$ : The same as for $n=78$ above.

$\mathbf{n}=67, H \cong 4^{2} D_{6},(|H|=96, i=64)$ : The same as for $n=80$ above.

$\mathbf{n}=66, H \cong 2{ }^{4} C_{6}(|H|=96, i=70)$ : The same as for $n=76$ above.

$\mathbf{n}=65, H \cong 2^{4} D_{6}(|H|=96, i=227): \operatorname{rk} N_{H}=18,\left(N_{H}\right)^{*} / N_{H} \cong \mathbb{Z} / 24 \mathbb{Z} \times \mathbb{Z} / 4 \mathbb{Z} \times$ $(\mathbb{Z} / 2 \mathbb{Z})^{2}$. Degenerations: $4 \mathbb{A}_{1}$ with $A_{S} \cong \mathbb{Z} / 12 \mathbb{Z} \times(\mathbb{Z} / 4 \mathbb{Z})^{2} ; 8 \mathbb{A}_{1}$ with $A_{S} \cong \mathbb{Z} / 24 \mathbb{Z} \times(\mathbb{Z} / 2 \mathbb{Z})^{2}$; $12 \mathbb{A}_{1}$ with $A_{S} \cong(\mathbb{Z} / 4 \mathbb{Z})^{3}$. $16 \mathbb{A}_{1}$ with $A_{S} \cong \mathbb{Z} / 12 \mathbb{Z} \times(\mathbb{Z} / 2 \mathbb{Z})^{2}$.

$\mathbf{n}=\mathbf{6 4}, H \cong 2^{4} C_{5}(|H|=80, i=49)$ : The same as for $n=81$ above.

$\mathbf{n}=63, H \cong M_{9}(|H|=72, i=41):$ rk $N_{H}=19$. No degenerations.

$\mathbf{n}=62, H \cong N_{72}(|H|=72, i=40):$ rk $N_{H}=19$. No degenerations.

$\mathbf{n}=\mathbf{6 1}, H \cong \mathfrak{A}_{4,3}(|H|=72, i=43): \operatorname{rk} N_{H}=18$ and $\left(N_{H}\right)^{*} / N_{H} \cong(\mathbb{Z} / 12 \mathbb{Z})^{2} \times \mathbb{Z} / 3 \mathbb{Z}$. Degenerations: $3 \mathbb{A}_{1}$ with $A_{S} \cong(\mathbb{Z} / 12 \mathbb{Z})^{2} \times \mathbb{Z} / 2 \mathbb{Z} ; 12 \mathbb{A}_{1}$ with $A_{S} \cong \mathbb{Z} / 24 \mathbb{Z} \times \mathbb{Z} / 3 \mathbb{Z}$.

$\mathbf{n}=60, H \cong \Gamma_{26} a_{2}(|H|=64, i=136)$ : The same as for $n=80$ above.

$\mathbf{n}=\mathbf{5 9}, H \cong \Gamma_{23} a_{2}(|H|=64, i=35)$ : The same as for $n=80$ above.

$\mathbf{n}=\mathbf{5 8}, H \cong \Gamma_{22} a_{1}(|H|=64, i=32)$ : The same as for $n=80$ above.

$\mathbf{n}=\mathbf{5 7}, H \cong \Gamma_{13} a_{1}(|H|=64, i=242)$ : The same as for $n=75$ above.

$\mathbf{n}=56, H \cong \Gamma_{25} a_{1}(|H|=64, i=138): \operatorname{rk} N_{H}=18,\left(N_{H}\right)^{*} / N_{H} \cong \mathbb{Z} / 8 \mathbb{Z} \times(\mathbb{Z} / 4 \mathbb{Z})^{3}$. Degenerations: $8 \mathbb{A}_{1}$ with $A_{S} \cong \mathbb{Z} / 8 \mathbb{Z} \times(\mathbb{Z} / 4 \mathbb{Z})^{2} ; 16 \mathbb{A}_{1}$ with $A_{S} \cong(\mathbb{Z} / 4 \mathbb{Z})^{3}$.

$\mathbf{n}=\mathbf{5 5}, H \cong \mathfrak{A}_{5}(|H|=60, i=5): \operatorname{rk} N_{H}=18$ and $\left(N_{H}\right)^{*} / N_{H} \cong \mathbb{Z} / 30 \mathbb{Z} \times \mathbb{Z} / 10 \mathbb{Z}$. Degenerations: $\mathbb{A}_{1}$ with $A_{S} \cong \mathbb{Z} / 30 \mathbb{Z} \times \mathbb{Z} / 10 \mathbb{Z} \times \mathbb{Z} / 2 \mathbb{Z} ; 5 \mathbb{A}_{1}$ with $A_{S} \cong \mathbb{Z} / 30 \mathbb{Z} \times(\mathbb{Z} / 2 \mathbb{Z})^{2}$; $6 \mathbb{A}_{1}$ with $A_{S} \cong \mathbb{Z} / 20 \mathbb{Z} \times \mathbb{Z} / 5 \mathbb{Z} ; 10 \mathbb{A}_{1}$ with $A_{S} \cong \mathbb{Z} / 60 \mathbb{Z} ; 15 \mathbb{A}_{1}$ with $A_{S} \cong \mathbb{Z} / 10 \mathbb{Z} \times(\mathbb{Z} / 2 \mathbb{Z})^{2}$.

$\mathbf{n}=\mathbf{5 4}, H \cong T_{48}(|H|=48, i=29): \operatorname{rk} N_{H}=19$ and $\left(N_{H}\right)^{*} / N_{H} \cong \mathbb{Z} / 24 \mathbb{Z} \times \mathbb{Z} / 8 \mathbb{Z} \times \mathbb{Z} / 2 \mathbb{Z}$. No degenerations.

$\mathbf{n}=53, H \cong 2^{2} Q_{12}(|H|=48, i=30)$ : The same as for $n=78$ above.

$\mathbf{n}=\mathbf{5 2}, H \cong 2^{2}\left(C_{2} \times C_{6}\right)(|H|=48, i=49)$ : The same as for $n=78$ above.

$\mathbf{n}=\mathbf{5 1}, H \cong C_{2} \times \mathfrak{S}_{4}(|H|=48, i=48): \operatorname{rk} N_{H}=18,\left(N_{H}\right)^{*} / N_{H} \cong(\mathbb{Z} / 12 \mathbb{Z})^{2} \times(\mathbb{Z} / 2 \mathbb{Z})^{2}$. Degenerations: $2 \mathbb{A}_{1}$ with $A_{S} \cong(\mathbb{Z} / 12 \mathbb{Z})^{2} \times \mathbb{Z} / 4 \mathbb{Z} ; 4 \mathbb{A}_{1}$ with $A_{S} \cong \mathbb{Z} / 24 \mathbb{Z} \times \mathbb{Z} / 6 \mathbb{Z} \times \mathbb{Z} / 2 \mathbb{Z}$; $6 \mathbb{A}_{1}$ with $A_{S} \cong \mathbb{Z} / 12 \mathbb{Z} \times(\mathbb{Z} / 4 \mathbb{Z})^{2} ; 8 \mathbb{A}_{1}$ with $A_{S} \cong \mathbb{Z} / 12 \mathbb{Z} \times \mathbb{Z} / 6 \mathbb{Z} \times \mathbb{Z} / 2 \mathbb{Z} ; 12 \mathbb{A}_{1}$ with $A_{S} \cong \mathbb{Z} / 24 \mathbb{Z} \times(\mathbb{Z} / 2 \mathbb{Z})^{2}$

$\mathbf{n}=\mathbf{5 0}, H \cong 4^{2} C_{3}(|H|=48, i=3)$ : The same as for $n=75$ above. 
$\mathbf{n}=49, H \cong 2{ }^{4} C_{3}(|H|=48, i=50): \operatorname{rk} N_{H}=17,\left(N_{H}\right)^{*} / N_{H} \cong \mathbb{Z} / 24 \mathbb{Z} \times(\mathbb{Z} / 2 \mathbb{Z})^{4}$. Degenerations of codimension 1 : $4 \mathbb{A}_{1}$ with $A_{S} \cong \mathbb{Z} / 12 \mathbb{Z} \times \mathbb{Z} / 4 \mathbb{Z} \times(\mathbb{Z} / 2 \mathbb{Z})^{2} ; 12 \mathbb{A}_{1}$ with $A_{S} \cong(\mathbb{Z} / 4 \mathbb{Z})^{2} \times(\mathbb{Z} / 2 \mathbb{Z})^{2} ; 16 \mathbb{A}_{1}$ with $A_{S} \cong \mathbb{Z} / 6 \mathbb{Z} \times(\mathbb{Z} / 2 \mathbb{Z})^{3}$.

$\mathbf{n}=\mathbf{4 8}, H \cong \mathfrak{S}_{3,3}(|H|=36, i=10): \operatorname{rk} N_{H}=18,\left(N_{H}\right)^{*} / N_{H} \cong \mathbb{Z} / 18 \mathbb{Z} \times \mathbb{Z} / 6 \mathbb{Z} \times(\mathbb{Z} / 3 \mathbb{Z})^{2}$. Degenerations: $3 \mathbb{A}_{1}$ with $A_{S} \cong \mathbb{Z} / 18 \mathbb{Z} \times(\mathbb{Z} / 6 \mathbb{Z})^{2} ; 6 \mathbb{A}_{1}$ with $A_{S} \cong \mathbb{Z} / 36 \mathbb{Z} \times(\mathbb{Z} / 3 \mathbb{Z})^{2} ; 9 \mathbb{A}_{1}$ with $A_{S} \cong(\mathbb{Z} / 6 \mathbb{Z})^{3}$.

$\mathbf{n}=\mathbf{4 7}, H \cong C_{3} \times \mathfrak{A}_{4}(|H|=36, i=11)$ : The same as for $n=61$ above.

$\mathbf{n}=\mathbf{4 6}, H \cong 3^{2} C_{4}(|H|=36, i=9): \operatorname{rk} N_{H}=18$ and $\left(N_{H}\right)^{*} / N_{H} \cong \mathbb{Z} / 18 \mathbb{Z} \times \mathbb{Z} / 6 \mathbb{Z} \times \mathbb{Z} / 3 \mathbb{Z}$. Degenerations: $6 \mathbb{A}_{1}$ with $A_{S} \cong \mathbb{Z} / 36 \mathbb{Z} \times \mathbb{Z} / 3 \mathbb{Z} ; 9 \mathbb{A}_{1}$ with $A_{S} \cong(\mathbb{Z} / 6 \mathbb{Z})^{2} \times \mathbb{Z} / 2 \mathbb{Z} ; 9 \mathbb{A}_{2}$ with $A_{S} \cong \mathbb{Z} / 6 \mathbb{Z} \times \mathbb{Z} / 3 \mathbb{Z}$.

$\mathbf{n}=\mathbf{4 5}, H \cong \Gamma_{6} a_{2}(|H|=32, i=44)$ : The same as for $n=80$ above.

$\mathbf{n}=\mathbf{4 4}, H \cong \Gamma_{3} e(|H|=32, i=11)$ : The same as for $n=80$ above.

$\mathbf{n}=\mathbf{4 3}, H \cong \Gamma_{7} a_{2}(|H|=32, i=7)$ : The same as for $n=80$ above.

$\left.\mathbf{n}=\mathbf{4 2}, H \cong \Gamma_{4} c_{2}|H|=32, i=31\right)$ : The same as for $n=75$ above.

$\mathbf{n}=\mathbf{4 1}, H \cong \Gamma_{7} a_{1},(|H|=32, i=6)$ : The same as for $n=56$ above.

$\mathbf{n}=\mathbf{4 0}, H \cong Q_{8} * Q_{8}(|H|=32, i=49): \operatorname{rk} N_{H}=17,\left(N_{H}\right)^{*} / N_{H} \cong(\mathbb{Z} / 4 \mathbb{Z})^{5}$. Degenerations of codimension 1 : $8 \mathbb{A}_{1}$ with $A_{S} \cong(\mathbb{Z} / 4 \mathbb{Z})^{4}$.

$\mathbf{n}=\mathbf{3 9}, H \cong 2^{4} C_{2}(|H|=32, i=27): \operatorname{rk} N_{H}=17,\left(N_{H}\right)^{*} / N_{H} \cong \mathbb{Z} / 8 \mathbb{Z} \times(\mathbb{Z} / 4 \mathbb{Z})^{2} \times$ $(\mathbb{Z} / 2 \mathbb{Z})^{2}$. Degenerations of codimension $1: 4 \mathbb{A}_{1}$ with $A_{S} \cong(\mathbb{Z} / 4 \mathbb{Z})^{4} ; 8 \mathbb{A}_{1}$ with $A_{S} \cong \mathbb{Z} / 8 \mathbb{Z} \times$ $\mathbb{Z} / 4 \mathbb{Z} \times(\mathbb{Z} / 2 \mathbb{Z})^{2} ; 16 \mathbb{A}_{1}$ with $A_{S} \cong(\mathbb{Z} / 4 \mathbb{Z})^{2} \times(\mathbb{Z} / 2 \mathbb{Z})^{2}$

$\mathbf{n}=\mathbf{3 8}, H \cong T_{24}(|H|=24, i=3)$ : The same as for $n=54$ above.

$\mathbf{n}=\mathbf{3 7}, H \cong T_{24},(|H|=24, i=3)$ : The same as for $n=77$ above.

$\mathbf{n}=\mathbf{3 6}, H \cong C_{3} \rtimes D_{8}(|H|=24, i=8)$ : The same as for $n=61$ above.

$\mathbf{n}=35, H \cong C_{2} \times \mathfrak{A}_{4},(|H|=24, i=13)$ : The same as for $n=51$ above.

$\mathbf{n}=\mathbf{3 4}, H \cong \mathfrak{S}_{4}(|H|=24, i=12): \operatorname{rk} N_{H}=17$ and $\left(N_{H}\right)^{*} / N_{H} \cong(\mathbb{Z} / 12 \mathbb{Z})^{2} \times \mathbb{Z} / 4 \mathbb{Z}$. Degenerations of codimension 1 : $\mathbb{A}_{1}$ with $A_{S} \cong(\mathbb{Z} / 12 \mathbb{Z})^{2} \times \mathbb{Z} / 4 \mathbb{Z} \times \mathbb{Z} / 2 \mathbb{Z} ; 2 \mathbb{A}_{1}$ with $A_{S} \cong$ $(\mathbb{Z} / 12 \mathbb{Z})^{2} \times(\mathbb{Z} / 2 \mathbb{Z})^{2} ; 3 \mathbb{A}_{1}$ with $A_{S} \cong \mathbb{Z} / 12 \mathbb{Z} \times(\mathbb{Z} / 4 \mathbb{Z})^{2} \times \mathbb{Z} / 2 \mathbb{Z} ; 4 \mathbb{A}_{1}$ with $A_{S} \cong \mathbb{Z} / 24 \mathbb{Z} \times \mathbb{Z} / 12 \mathbb{Z} ;$ $6 \mathbb{A}_{1}$ with $A_{S} \cong \mathbb{Z} / 12 \mathbb{Z} \times \mathbb{Z} / 4 \mathbb{Z} \times(\mathbb{Z} / 2 \mathbb{Z})^{2} ; 8 \mathbb{A}_{1}$ with $A_{S} \cong(\mathbb{Z} / 12 \mathbb{Z})^{2} ; 12 \mathbb{A}_{1}$ with $A_{S} \cong$ $\mathbb{Z} / 24 \mathbb{Z} \times \mathbb{Z} / 4 \mathbb{Z} ; 6 \mathbb{A}_{2}$ with $A_{S} \cong \mathbb{Z} / 12 \mathbb{Z} \times \mathbb{Z} / 4 \mathbb{Z}$

$\mathbf{n}=33, H \cong C_{7} \rtimes C_{3}(|H|=21, i=1): \operatorname{rk} N_{H}=18$ and $\left(N_{H}\right)^{*} / N_{H} \cong(\mathbb{Z} / 7 \mathbb{Z})^{3}$. Degenerations: $7 \mathbb{A}_{1}$ with $A_{S} \cong \mathbb{Z} / 14 \mathbb{Z} \times \mathbb{Z} / 7 \mathbb{Z}$. 
$\mathbf{n}=32, H \cong H o l\left(C_{5}\right)(|H|=20, i=3): \operatorname{rk} N_{H}=18$ and $\left(N_{H}\right)^{*} / N_{H} \cong(\mathbb{Z} / 10 \mathbb{Z})^{2} \times \mathbb{Z} / 5 \mathbb{Z}$. Degenerations: $2 \mathbb{A}_{1}$ with $A_{S} \cong \mathbb{Z} / 20 \mathbb{Z} \times(\mathbb{Z} / 5 \mathbb{Z})^{2} ; 5 \mathbb{A}_{1}$ with $A_{S} \cong(\mathbb{Z} / 10 \mathbb{Z})^{2} \times \mathbb{Z} / 2 \mathbb{Z} ; 10 \mathbb{A}_{1}$ with $A_{S} \cong \mathbb{Z} / 20 \mathbb{Z} \times \mathbb{Z} / 5 \mathbb{Z} ; 5 \mathbb{A}_{2}$ with $A_{S} \cong \mathbb{Z} / 10 \mathbb{Z} \times \mathbb{Z} / 5 \mathbb{Z}$.

$\mathbf{n}=\mathbf{3 1}, H \cong C_{3} \times D_{6}(|H|=18, i=3)$ : The same as for $n=48$ above.

$\mathbf{n}=30, H \cong \mathfrak{A}_{3,3}(|H|=18, i=4): \operatorname{rk} N_{H}=16$ and $\left(N_{H}\right)^{*} / N_{H} \cong \mathbb{Z} / 9 \mathbb{Z} \times(\mathbb{Z} / 3 \mathbb{Z})^{4}$. Degenerations of codimension 1 : $3 \mathbb{A}_{1}$ with $A_{S} \cong \mathbb{Z} / 18 \mathbb{Z} \times(\mathbb{Z} / 3 \mathbb{Z})^{3} ; 9 \mathbb{A}_{1}$ with $A_{S} \cong \mathbb{Z} / 6 \mathbb{Z} \times$ $(\mathbb{Z} / 3 \mathbb{Z})^{3}$.

$\mathbf{n}=\mathbf{2 9}, H \cong Q_{16}(|H|=16, i=9)$ : The same as for $n=80$ above.

$\mathbf{n}=\mathbf{2 8}, H \cong \Gamma_{2} d(|H|=16, i=6)$ : The same as for $n=80$ above.

$\mathbf{n}=\mathbf{2 7}, H \cong C_{2} \times Q_{8}(|H|=16, i=12)$ : The same as for $n=75$ above.

$\mathbf{n}=\mathbf{2 6}, H \cong S D_{16}(|H|=16, i=8): \operatorname{rk} N_{H}=18$ and $\left(N_{H}\right)^{*} / N_{H} \cong(\mathbb{Z} / 8 \mathbb{Z})^{2} \times \mathbb{Z} / 4 \mathbb{Z} \times$ $\mathbb{Z} / 2 \mathbb{Z}$. Degenerations: $8 \mathbb{A}_{1}$ with $A_{S} \cong \mathbb{Z} / 16 \mathbb{Z} \times \mathbb{Z} / 4 \mathbb{Z} \times \mathbb{Z} / 2 \mathbb{Z} ; 2 \mathbb{A}_{2}$ with $A_{S} \cong(\mathbb{Z} / 8 \mathbb{Z})^{2} \times \mathbb{Z} / 2 \mathbb{Z}$.

$\mathbf{n}=\mathbf{2 5}, H \cong C_{4}^{2}(|H|=16, i=2)$ : The same as for $n=75$ above.

$\mathbf{n}=\mathbf{2 4}, H \cong Q_{8} * C_{4}(|H|=16, i=13)$ : The same as for $n=40$ above.

$\mathbf{n}=\mathbf{2 3}, H \cong \Gamma_{2} c_{1}(|H|=16, i=3)$ : The same as for $n=39$ above.

$\mathbf{n}=\mathbf{2 2}, H \cong C_{2} \times D_{8}(|H|=16, i=11): \operatorname{rk} N_{H}=16,\left(N_{H}\right)^{*} / N_{H} \cong(\mathbb{Z} / 4 \mathbb{Z})^{4} \times(\mathbb{Z} / 2 \mathbb{Z})^{2}$. Degenerations of codimension 1 : $2 \mathbb{A}_{1}$ with $A_{S} \cong(\mathbb{Z} / 4 \mathbb{Z})^{5} ; 4 \mathbb{A}_{1}$ with $A_{S} \cong \mathbb{Z} / 8 \mathbb{Z} \times(\mathbb{Z} / 4 \mathbb{Z})^{2} \times$ $(\mathbb{Z} / 2 \mathbb{Z})^{2} ; 8 \mathbb{A}_{1}$ with $A_{S} \cong(\mathbb{Z} / 4 \mathbb{Z})^{3} \times(\mathbb{Z} / 2 \mathbb{Z})^{2}$.

$\mathbf{n}=\mathbf{2 1}, H \cong C_{2}^{4}(|H|=16, i=14): \operatorname{rk} N_{H}=15,\left(N_{H}\right)^{*} / N_{H} \cong \mathbb{Z} / 8 \mathbb{Z} \times(\mathbb{Z} / 2 \mathbb{Z})^{6}$. Degenerations of codimension 1 : $4 \mathbb{A}_{1}$ with $A_{S} \cong(\mathbb{Z} / 4 \mathbb{Z})^{2} \times(\mathbb{Z} / 2 \mathbb{Z})^{4} ; 16 \mathbb{A}_{1}$ with $A_{S} \cong$ $(\mathbb{Z} / 2 \mathbb{Z})^{6}$.

$\mathbf{n}=\mathbf{2 0}, H \cong Q_{12}(|H|=12, i=1)$ : The same as for $n=61$ above.

$\mathbf{n}=19, H \cong C_{2} \times C_{6}(|H|=12, i=5)$ : The same as for $n=61$ above.

$\mathbf{n}=\mathbf{1 8}, H \cong D_{12}(|H|=12, i=4): \operatorname{rk} N_{H}=16$ and $\left(N_{H}\right)^{*} / N_{H} \cong(\mathbb{Z} / 6 \mathbb{Z})^{4}$. Degenerations of codimension 1 : $\mathbb{A}_{1}$ with $A_{S} \cong(\mathbb{Z} / 6 \mathbb{Z})^{4} \times \mathbb{Z} / 2 \mathbb{Z} ; 2 \mathbb{A}_{1}$ with $A_{S} \cong \mathbb{Z} / 12 \mathbb{Z} \times(\mathbb{Z} / 6 \mathbb{Z})^{2} \times$ $\mathbb{Z} / 3 \mathbb{Z} ; 3 \mathbb{A}_{1}$ with $A_{S} \cong(\mathbb{Z} / 6 \mathbb{Z})^{3} \times(\mathbb{Z} / 2 \mathbb{Z})^{2} ; 6 \mathbb{A}_{1}$ with $A_{S} \cong \mathbb{Z} / 12 \mathbb{Z} \times(\mathbb{Z} / 6 \mathbb{Z})^{2}$.

$\mathbf{n}=\mathbf{1 7}, H \cong \mathfrak{A}_{4}(|H|=12, i=3): \operatorname{rk} N_{H}=16$ and $\left(N_{H}\right)^{*} / N_{H} \cong(\mathbb{Z} / 12 \mathbb{Z})^{2} \times(\mathbb{Z} / 2 \mathbb{Z})^{2}$. Degenerations of codimension 1 : $\mathbb{A}_{1}$ with $A_{S} \cong(\mathbb{Z} / 12 \mathbb{Z})^{2} \times(\mathbb{Z} / 2 \mathbb{Z})^{3} ; 3 \mathbb{A}_{1}$ with $A_{S} \cong \mathbb{Z} / 12 \mathbb{Z} \times$ $\mathbb{Z} / 4 \mathbb{Z} \times(\mathbb{Z} / 2 \mathbb{Z})^{3} ; 4 \mathbb{A}_{1}$ with $A_{S} \cong \mathbb{Z} / 24 \mathbb{Z} \times \mathbb{Z} / 6 \mathbb{Z} \times \mathbb{Z} / 2 \mathbb{Z} ; 6 \mathbb{A}_{1}$ with $A_{S} \cong \mathbb{Z} / 12 \mathbb{Z} \times(\mathbb{Z} / 4 \mathbb{Z})^{2} ;$ $12 \mathbb{A}_{1}$ with $A_{S} \cong \mathbb{Z} / 24 \mathbb{Z} \times(\mathbb{Z} / 2 \mathbb{Z})^{2}$.

$\mathbf{n}=\mathbf{1 6}, H \cong D_{10}(|H|=10, i=1): \operatorname{rk} N_{H}=16$ and $\left(N_{H}\right)^{*} / N_{H} \cong(\mathbb{Z} / 5 \mathbb{Z})^{4}$. Degenerations of codimension 1 : $\mathbb{A}_{1}$ with $A_{S} \cong \mathbb{Z} / 10 \mathbb{Z} \times(\mathbb{Z} / 5 \mathbb{Z})^{3} ; 5 \mathbb{A}_{1}$ with $A_{S} \cong \mathbb{Z} / 10 \mathbb{Z} \times(\mathbb{Z} / 5 \mathbb{Z})^{2}$. 
$\mathbf{n}=\mathbf{1 5}, H \cong C_{3}^{2}(|H|=9, i=2)$ : The same as for $n=30$ above.

$\mathbf{n}=\mathbf{1 4}, H \cong C_{8}(|H|=8, i=1)$ : The same as for $n=26$ above.

$\mathbf{n}=\mathbf{1 3}, H \cong Q_{8}(|H|=8, i=4)$ : The same as for $n=40$ above.

$\mathbf{n}=\mathbf{1 2}, H \cong Q_{8}(|H|=8, i=4): \operatorname{rk} N_{H}=17,\left(N_{H}\right)^{*} / N_{H} \cong(\mathbb{Z} / 8 \mathbb{Z})^{2} \times(\mathbb{Z} / 2 \mathbb{Z})^{3}$. Degenerations of codimension 1 : $8 \mathbb{A}_{1}$ with $A_{S} \cong \mathbb{Z} / 16 \mathbb{Z} \times(\mathbb{Z} / 2 \mathbb{Z})^{3} ; \mathbb{A}_{2}$ with $A_{S} \cong(\mathbb{Z} / 8 \mathbb{Z})^{2} \times$ $(\mathbb{Z} / 2 \mathbb{Z})^{2}$.

$\mathbf{n}=\mathbf{1 1}, H \cong C_{2} \times C_{4}(|H|=8, i=2)$ : The same as for $n=22$ above.

$\mathbf{n}=\mathbf{1 0}, H \cong D_{8}(|H|=8, i=3): \operatorname{rk} N_{H}=15$ and $\left(N_{H}\right)^{*} / N_{H} \cong(\mathbb{Z} / 4 \mathbb{Z})^{5}$. Degenerations of codimension 1 : $\mathbb{A}_{1}$ with $A_{S} \cong(\mathbb{Z} / 4 \mathbb{Z})^{5} \times \mathbb{Z} / 2 \mathbb{Z} ; 2 \mathbb{A}_{1}$ with $A_{S} \cong(\mathbb{Z} / 4 \mathbb{Z})^{4} \times(\mathbb{Z} / 2 \mathbb{Z})^{2} ; 4 \mathbb{A}_{1}$ with $A_{S} \cong \mathbb{Z} / 8 \mathbb{Z} \times(\mathbb{Z} / 4 \mathbb{Z})^{3} ; 8 \mathbb{A}_{1}$ with $A_{S} \cong(\mathbb{Z} / 4 \mathbb{Z})^{4} ; 2 \mathbb{A}_{2}$ with $A_{S} \cong(\mathbb{Z} / 4 \mathbb{Z})^{4}$.

$\mathbf{n}=\mathbf{9}, H \cong C_{2}^{3}(|H|=8, i=5): \operatorname{rk} N_{H}=14,\left(N_{H}\right)^{*} / N_{H} \cong(\mathbb{Z} / 4 \mathbb{Z})^{2} \times(\mathbb{Z} / 2 \mathbb{Z})^{6}$. Degenerations of codimension 1 : $2 \mathbb{A}_{1}$ with $A_{S} \cong(\mathbb{Z} / 4 \mathbb{Z})^{3} \times(\mathbb{Z} / 2 \mathbb{Z})^{4} ; 4 \mathbb{A}_{1}$ with $A_{S} \cong \mathbb{Z} / 8 \mathbb{Z} \times$ $(\mathbb{Z} / 2 \mathbb{Z})^{6} ; 8 \mathbb{A}_{1}$ with $A_{S} \cong \mathbb{Z} / 4 \mathbb{Z} \times(\mathbb{Z} / 2 \mathbb{Z})^{6}$.

$\mathbf{n}=\mathbf{8}, H \cong C_{7}(|H|=7, i=1)$ : The same as for $n=33$ above.

$\mathbf{n}=7, H \cong C_{6}(|H|=6, i=2)$ : The same as for $n=18$ above.

$\mathbf{n}=6, H \cong D_{6}(|H|=6, i=1): \operatorname{rk} N_{H}=14$ and $\left(N_{H}\right)^{*} / N_{H} \cong(\mathbb{Z} / 6 \mathbb{Z})^{2} \times(\mathbb{Z} / 3 \mathbb{Z})^{3}$. Degenerations of codimension 1 : $\mathbb{A}_{1}$ with $A_{S} \cong(\mathbb{Z} / 6 \mathbb{Z})^{3} \times(\mathbb{Z} / 3 \mathbb{Z})^{2} ; 2 \mathbb{A}_{1}$ with $A_{S} \cong \mathbb{Z} / 12 \mathbb{Z} \times$ $(\mathbb{Z} / 3 \mathbb{Z})^{4} ; 3 \mathbb{A}_{1}$ with $A_{S} \cong(\mathbb{Z} / 6 \mathbb{Z})^{3} \times \mathbb{Z} / 3 \mathbb{Z} ; 6 \mathbb{A}_{1}$ with $A_{S} \cong \mathbb{Z} / 12 \mathbb{Z} \times(\mathbb{Z} / 3 \mathbb{Z})^{3}$.

$\mathbf{n}=\mathbf{5}, H \cong C_{5}(|H|=5, i=1)$ : The same as for $n=16$ above.

$\mathbf{n}=4, H \cong C_{4}(|H|=4, i=1): \operatorname{rk} N_{H}=14$ and $\left(N_{H}\right)^{*} / N_{H} \cong(\mathbb{Z} / 4 \mathbb{Z})^{4} \times(\mathbb{Z} / 2 \mathbb{Z})^{2}$. Degenerations of codimension 1 : $\mathbb{A}_{1}$ with $A_{S} \cong(\mathbb{Z} / 4 \mathbb{Z})^{4} \times(\mathbb{Z} / 2 \mathbb{Z})^{3} ; 2 \mathbb{A}_{1}$ with $A_{S} \cong(\mathbb{Z} / 4 \mathbb{Z})^{5}$; $4 \mathbb{A}_{1}$ with $A_{S} \cong \mathbb{Z} / 8 \mathbb{Z} \times(\mathbb{Z} / 4 \mathbb{Z})^{2} \times(\mathbb{Z} / 2 \mathbb{Z})^{2} ; \mathbb{A}_{2}$ with $A_{S} \cong(\mathbb{Z} / 4 \mathbb{Z})^{4} \times \mathbb{Z} / 2 \mathbb{Z} ;$

$\mathbf{n}=\mathbf{3}, H \cong C_{2}^{2}(|H|=4, i=2): \operatorname{rk} N_{H}=12$ and $\left(N_{H}\right)^{*} / N_{H} \cong(\mathbb{Z} / 4 \mathbb{Z})^{2} \times(\mathbb{Z} / 2 \mathbb{Z})^{6}$. Degenerations of codimension 1 : $\mathbb{A}_{1}$ with $A_{S} \cong(\mathbb{Z} / 4 \mathbb{Z})^{2} \times(\mathbb{Z} / 2 \mathbb{Z})^{7} ; 2 \mathbb{A}_{1}$ with $A_{S} \cong(\mathbb{Z} / 4 \mathbb{Z})^{3} \times$ $(\mathbb{Z} / 2 \mathbb{Z})^{4} ; 4 \mathbb{A}_{1}$ with $A_{S} \cong \mathbb{Z} / 8 \mathbb{Z} \times(\mathbb{Z} / 2 \mathbb{Z})^{6}$.

$\mathbf{n}=\mathbf{2}, H \cong C_{3}(|H|=3, i=1): \operatorname{rk} N_{H}=12$ and $\left(N_{H}\right)^{*} / N_{H} \cong(\mathbb{Z} / 3 \mathbb{Z})^{6}$. Degenerations of codimension 1 : $\mathbb{A}_{1}$ with $A_{S} \cong \mathbb{Z} / 6 \mathbb{Z} \times(\mathbb{Z} / 3 \mathbb{Z})^{5} ; 3 \mathbb{A}_{1}$ with $A_{S} \cong \mathbb{Z} / 6 \mathbb{Z} \times(\mathbb{Z} / 3 \mathbb{Z})^{4}$.

$\mathbf{n}=\mathbf{1}, H \cong C_{2}(|H|=2, i=1): \operatorname{rk} N_{H}=8$ and $\left(N_{H}\right)^{*} / N_{H} \cong(\mathbb{Z} / 2 \mathbb{Z})^{8}$. Degenerations of codimension 1: $\mathbb{A}_{1}$ with $A_{S} \cong(\mathbb{Z} / 2 \mathbb{Z})^{9} ; 2 \mathbb{A}_{1}$ with $A_{S} \cong \mathbb{Z} / 4 \mathbb{Z} \times(\mathbb{Z} / 2 \mathbb{Z})^{6}$. 


\section{Conjecture and some remarks.}

Theorem 3 shows that for a fixed type (defined by $n$ ) of abstract finite symplectic group of automorphisms of K3 and for a fixed type of degeneration of codimension 1 (type of Dynkin diagram), the discriminant group $A_{S}$ of the corresponding lattice $S$ is always the same. It is natural to suppose that the more strong statement is valid: that the isomorphism class of the lattice $S$ is defined uniquely. But, exact calculations and considerations show that it is valid only with some few exceptions given in the Conjecture below.

Conjecture 4. For a fixed type (defined by $n$ ) of abstract finite symplectic group $H$ of automorphisms of Kählerian K3 surfaces and for a fixed type of degeneration of codimension 1 (type of Dynkin diagram) of Kählerian K3 surfaces, the corresponding lattice $S=\left[N_{H}, \alpha_{i, j}\right]_{p r}$ is unique up to isomorphisms of lattices.

The same can be formulated geometrically. Let $X$ be a Kählerian K3 surface with $S_{X}<0$. Let $H=A u t^{0} X$ be the group of symplectic automorphisms of $X$ and $P(X)$ be a the set of classes of non-singular rational curves on $X$. Let $N_{H}=\left(\left(S_{X}\right)^{H}\right)_{S_{X}}^{\perp}$ be the coinvariant sublattice. Assume that $S_{X}$ is generated by $N_{H}$ and $P(X)$ up to finite index, and $\mathrm{rk} S_{X}=$ $\mathrm{rk} N_{H}+1$. Then the isomorphism class of the lattice $S=S_{X}$ is defined uniquely by the type of $H$ as abstract group (equivalent to $n$ ) and the type of the Dynkin diagram of $P(X)$.

But, it is valid with the following and only the following two exceptions:

(1) $\mathbf{n}=\mathbf{3 4}$ (equivalently, $H \cong \mathfrak{S}_{4}$ ) and the degeneration of the type $6 \mathbb{A}_{1}$;

(2) $\mathbf{n}=\mathbf{1 0}$ (equivalently, $H \cong D_{8}$ ) and the degeneration of the type $2 \mathbb{A}_{1}$.

In both these cases there are exactly two isomorphism classes of the lattices $S$.

Obviously, the conjecture is valid for a trivial $H$. Theorem 3 shows that the conjecture is valid for the discriminant group $A_{S}$ of the lattice $S$. We hope to present proofs of Conjecture 4 and of more delicate statements in further publications. They will give classification of degenerations of codimension one of Kählerian K3 surfaces with finite symplectic automorphism groups (not of types of degenerations only).

From Theorems 2 and 3 we also obtain:

Corollary 5. All types of degenerations of codimension 1 of Kählerian K3 surfaces with finite symplectic automorphism groups defined by the invariant $n$ of the group and by the type of Dynkin diagram can be obtained from markings by Niemeier lattices $N_{23}=N\left(24 A_{1}\right)$ and $N_{22}=N\left(12 A_{2}\right)$. Degenerations of codimension 1 marked by other Niemeier lattices $N_{j}$, $j=1, \ldots 21$, give the same types of degenerations. 


\section{Appendix: Programs}

Here we give Programs 5 and 6 for GP/PARI Calculator, Version 2.7.0 which were used for calculations above. They also include Programs 1 - 4 from [17] and [18].

Program5: niemeier \general2a.txt

$\backslash \backslash$ for a Niemeier lattice $\mathrm{N} \_$given by root matrix $\mathrm{r}$

$\backslash$ \and cord matrix cord

$\backslash \backslash$ and subgroup $\mathrm{H} \backslash$ subset $\mathrm{A}(\mathrm{N} \mathrm{i})$

$\backslash \backslash$ and its orbits ORB matrix, each line gives

$\backslash$ \orbit of length $>1$ with 0 remaining elements

$\backslash \backslash$ it calculates all additional

$\backslash \backslash 1$-elements orbits to matrix ORBF and prints them

$\backslash \backslash$ (1-elements orbits the last)

$\backslash$ \it calculates coinvariant sublattice N_H

$\backslash \backslash$ by its rational generators, and checks if

$\backslash \backslash$ N_H has primitive embedding to L_K3

$\backslash \backslash$ Then it prints invariants of the discriminant group of N_H

$\mathrm{sORB}=$ matsize $(\mathrm{ORB}) ; \mathrm{m} 1=0$;

for $(\mathrm{k} 1=1, \mathrm{sORB}[1]$, for $(\mathrm{k} 2=1, \mathrm{sORB}[2], \backslash$

$\operatorname{if}(\mathrm{ORB}[\mathrm{k} 1, \mathrm{k} 2]==0, \mathrm{~m} 1=\mathrm{m} 1+1)))$;

$\mathrm{ORBF}=\operatorname{matrix}(\mathrm{sORB}[1]+(24-\mathrm{m} 1), \mathrm{sORB}[2])$;

for $(\mathrm{k}=1, \mathrm{sORB}[1], \mathrm{ORBF}[\mathrm{k}]=,\mathrm{ORB}[\mathrm{k}],) ; \mathrm{l}=\mathrm{sORB}[1]$;

for $(\mathrm{t}=1,24, \mathrm{mu}=0$; for $(\mathrm{k} 1=1, \mathrm{sORB}[1]$,for $(\mathrm{k} 2=1, \mathrm{sORB}[2]$,

if $(\mathrm{ORB}[\mathrm{k} 1, \mathrm{k} 2] !=\mathrm{t}, \mathrm{mu}=1))) ; \mathrm{if}(\mathrm{mu}==1, \mathrm{l}=\mathrm{l}+1 ; \mathrm{ORBF}[\mathrm{l}, 1]=\mathrm{t})) ;$

print $(" \mathrm{ORBF}=", \mathrm{ORBF}) ; \backslash$

SUBL0 = matrix $(24,24) ;$ alpha $=0 ; \backslash$

for $(\mathrm{k} 1=1, \mathrm{sORB}[1]$,for $(\mathrm{k} 2=1, \mathrm{sORB}[2]-1, \backslash$

if $(\mathrm{ORB}[\mathrm{k} 1, \mathrm{k} 2+1]>0$, alpha $=$ alpha $+1 ; \backslash$

SUBL0[,alpha $]=\mathrm{R}[, \mathrm{ORB}[\mathrm{k} 1, \mathrm{k} 2]]-\mathrm{R}[, \mathrm{ORB}[\mathrm{k} 1, \mathrm{k} 2+1]]))) ; \backslash$

$\mathrm{sORBF}=$ matsize $(\mathrm{ORBF}) ;$

$\mathrm{SUBL}=\mathrm{SUBL} 0 ; \mathrm{a}=\operatorname{matrix}(24,24+$ matsize $($ cord $)[1]) ; \backslash$

for $(\mathrm{i}=1,24, \mathrm{a}[\mathrm{i}, \mathrm{i}]=1) ; \operatorname{for}(\mathrm{i}=1, \operatorname{matsize}(\operatorname{cord})[1], \mathrm{a}[, 24+\mathrm{i}]=\operatorname{cord}[\mathrm{i}, \sim \sim) ;$

$\mathrm{L}=\mathrm{a} ; \mathrm{N}=\mathrm{SUBL} ; \mathrm{ggg}=\operatorname{gcd}(\mathrm{N}) ; \mathrm{N} 1=\mathrm{N} / \operatorname{ggg} ; \mathrm{M}=\mathrm{L} ; \mathrm{gg}=\operatorname{gcd}(\mathrm{M}) ; \mathrm{M} 1=\mathrm{M} / \operatorname{gg} ; \backslash$

$\mathrm{ww}=\operatorname{matsnf}(\mathrm{M} 1,1) ; \mathrm{uu}=\mathrm{ww}[1] ; \mathrm{vv}=\mathrm{ww}[2] ; \mathrm{dd}=\mathrm{ww}[3] ; \backslash$

$\mathrm{mm}=\operatorname{matsize}(\mathrm{dd})[1] ; \mathrm{nn}=\operatorname{matsize}(\mathrm{dd})[2] ; \backslash$

$\mathrm{nnn}=\mathrm{nn} ;$ for $(\mathrm{i}=1, \mathrm{nn}, \mathrm{if}(\mathrm{dd}[, \mathrm{i}]==0, \mathrm{nnn}=\mathrm{nnn}-1)) ; \mathrm{VV}=\operatorname{matrix}(\mathrm{nn}, \mathrm{nnn}) ; \backslash$

$\mathrm{nnnn}=0 ;$ for $(\mathrm{i}=1, \mathrm{nn}, \mathrm{if}(\mathrm{dd}[, \mathrm{i}]==0, \mathrm{nnnn}=\mathrm{nnnn}+1 ; \mathrm{VV}[, \mathrm{nnnn}]=\mathrm{vv}[, \mathrm{i}])) ;$ 


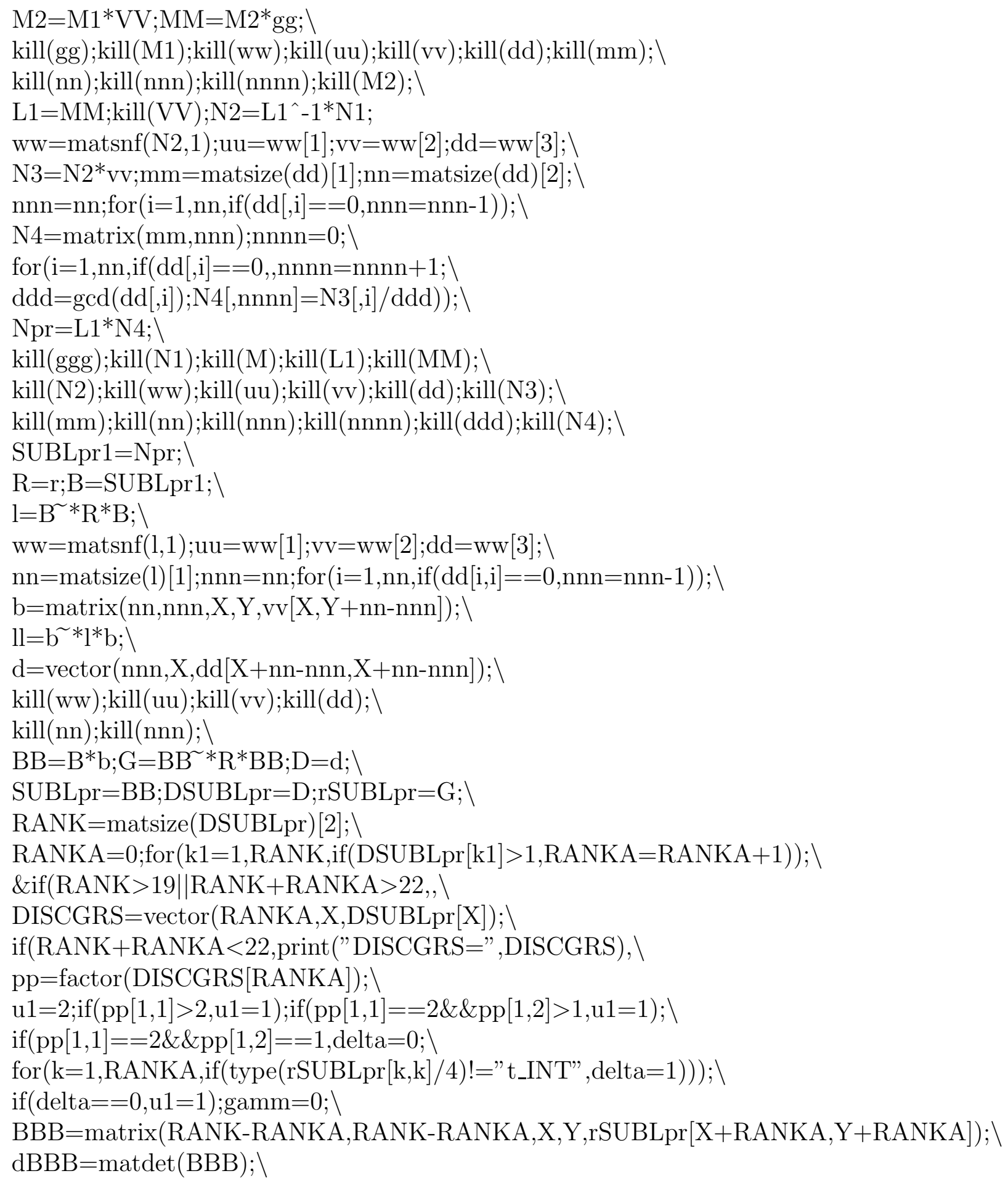


for $(\mathrm{t} 1=\mathrm{u} 1$, matsize $(\mathrm{pp})[1], \mathrm{if}(\mathrm{pp}[\mathrm{t} 1,1]==2 \& \& \backslash$

$\operatorname{Mod}(\mathrm{dBBB}, 8) !=\operatorname{Mod}(1,8) \& \& \operatorname{Mod}(\mathrm{dBBB}, 8) !=\operatorname{Mod}(-1,8), \operatorname{gamm}=1) ; \backslash$

if(pp[t1,1]!=2\&\&kronecker((-1)^(RANK+1)*dBBB,pp[t1,1])!=1,gamm=1)); \

if $($ gamm $==0$, print ("DISCGRS $=$, DISCGRS $)))$;

Program 6: niemeier \degen1a.txt

$\backslash \backslash$ for a Niemeier lattice N_i given by root matrix $\mathrm{r}$

$\backslash$ land cord matrix cord

$\backslash$ \and sugroup $\mathrm{H} \backslash$ subset $\mathrm{A}\left(\mathrm{N} \_\mathrm{i}\right)$

$\backslash \backslash$ and its orbits ORB matrix, each line gives orbit of length $>1$,

$\backslash$ \with 0 remaining elements

$\backslash \backslash$ it calculates all additional

$\backslash \backslash 1$-elements orbits to matrix ORBF and prints them

$\backslash \backslash$ (1-elements orbits the last)

$\backslash \backslash$ it calculates coinvariant sublattice N_H by its rational generators,

$\backslash \backslash$ for each first element k_i of i line of ORBF

$\backslash \backslash$ it calculates invariants of the degeneration

\| sublattice $\mathrm{N}(\mathrm{H}, \mathrm{i})=\left[\mathrm{N} \_\mathrm{H}, \mathrm{i}\right]$ using program general1.txt

$\backslash \backslash$ and checks if

$\backslash \backslash \mathrm{N}(\mathrm{H}, \mathrm{i})$ has primitive embedding to $\mathrm{L} \_\mathrm{K} 3$

$\backslash \backslash$ Then it prints k_i and invariants of the discriminant

I group of $\mathrm{N}(\mathrm{H}, \mathrm{i})$

$\backslash$ \it prints: k_i,

$\backslash$ \and invariants of $\mathrm{N}\left(\mathrm{H}_{-} \mathrm{i}\right) \backslash$ subset $\mathrm{N}\left(\mathrm{H}_{-} \mathrm{i}\right)^{\wedge} \backslash$ ast

$\mathrm{sORB}=$ matsize $(\mathrm{ORB}) ; \mathrm{m} 1=0$;

for $(\mathrm{k} 1=1, \mathrm{sORB}[1]$,for $(\mathrm{k} 2=1, \mathrm{sORB}[2], \backslash$

if $(\mathrm{ORB}[\mathrm{k} 1, \mathrm{k} 2]==0, \mathrm{~m} 1=\mathrm{m} 1+1)))$;

$\mathrm{ORBF}=\operatorname{matrix}(\mathrm{sORB}[1]+(24-\mathrm{m} 1), \mathrm{sORB}[2])$;

for $(\mathrm{k}=1, \mathrm{sORB}[1], \mathrm{ORBF}[\mathrm{k}]=,\mathrm{ORB}[\mathrm{k}],) ; \mathrm{l}=\mathrm{sORB}[1]$;

for $(\mathrm{t}=1,24, \mathrm{mu}=0 ;$ for $(\mathrm{k} 1=1, \mathrm{sORB}[1]$,for $(\mathrm{k} 2=1, \mathrm{sORB}[2], \backslash$

if $(\mathrm{ORB}[\mathrm{k} 1, \mathrm{k} 2]$ ! $=\mathrm{t}, \mathrm{mu}=1))) ; \mathrm{if}(\mathrm{mu}==1, \mathrm{l}=\mathrm{l}+1 ; \mathrm{ORBF}[\mathrm{l}, 1]=\mathrm{t})) ; \backslash$

print("ORBF=",ORBF);

SUBL0 $=\operatorname{matrix}(24,24) ;$ alpha $=0 ; \backslash$

for $(\mathrm{k} 1=1, \mathrm{sORB}[1]$,for $(\mathrm{k} 2=1, \mathrm{sORB}[2]-1, \backslash$

if $(\mathrm{ORB}[\mathrm{k} 1, \mathrm{k} 2+1]>0$, alpha $=$ alpha $+1 ; \backslash$

SUBL0[,alpha $]=\mathrm{R}[, \mathrm{ORB}[\mathrm{k} 1, \mathrm{k} 2]]-\mathrm{R}[, \mathrm{ORB}[\mathrm{k} 1, \mathrm{k} 2+1]]))) ; \backslash$

$\mathrm{sORBF}=$ matsize $(\mathrm{ORBF}) ;$

for $(\mathrm{n}=1, \mathrm{sORBF}[1], \mathrm{t}=\mathrm{ORBF}[\mathrm{n}, 1] ; \mathrm{SUBL}=\mathrm{SUBL} 0 ; \mathrm{SUBL}[\mathrm{t}, \mathrm{alpha}+1]=1 ; \backslash$ 


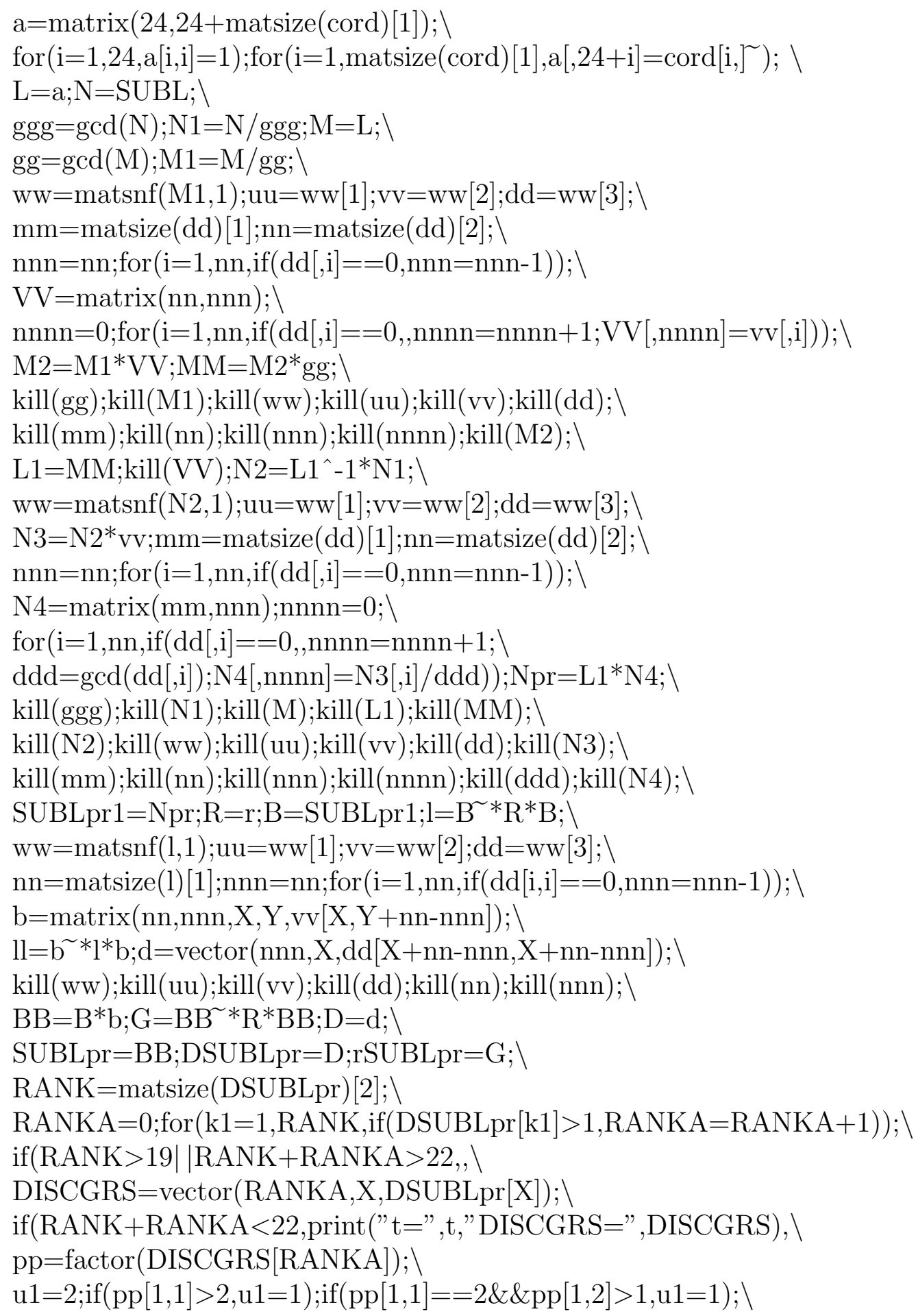


if $(\operatorname{pp}[1,1]==2 \& \& p p[1,2]==1$, delta $=0 ; \backslash$

for $(\mathrm{k}=1, \mathrm{RANKA}, \mathrm{if}(\mathrm{type}(\mathrm{rSUBLpr}[\mathrm{k}, \mathrm{k}] / 4) !=$ t_INT",delta=1) $)) ;$

if $($ delta $==0, \mathrm{u} 1=1)$;gamm $=0 ; \nmid$

$\mathrm{BBB}=$ matrix (RANK-RANKA,RANK-RANKA,X,Y,rSUBLpr[X+RANKA,Y+RANKA $]) ;$ $\mathrm{dBBB}=\operatorname{matdet}(\mathrm{BBB}) ;$

for $(\mathrm{t} 1=\mathrm{u} 1$, matsize $(\mathrm{pp})[1], \mathrm{if}(\mathrm{pp}[\mathrm{t} 1,1]==2 \& \& \backslash$

$\operatorname{Mod}(\mathrm{dBBB}, 8) !=\operatorname{Mod}(1,8) \& \& \operatorname{Mod}(\mathrm{dBBB}, 8) !=\operatorname{Mod}(-1,8), \operatorname{gamm}=1) ; \backslash$

if $\left(\operatorname{pp}[\mathrm{t} 1,1] !=2 \& \&\right.$ kronecker $\left((-1)^{\wedge}(\mathrm{RANK}+1)^{*} \mathrm{dBBB}, \mathrm{pp}[\mathrm{t} 1,1]\right) !=1$, gamm=1));

if $($ gamm $==0, \operatorname{print}(" \mathrm{t}=$, t) $)$ print("DISCGRS=",DISCGRS $))))$ );

\section{References}

[1] W. Barth, K3 surfaces with nine cusps, Preprint 1997, arXiv:alg-geom/9709031, 9 pages.

[2] N. Bourbaki, Groupes et algèbres de Lie, Groupes de Coxeter et systèmes de Tits, Groupes engendres par des reflexions, systèmes de racines, Hermann, Paris VI, 1968.

[3] E. Brieskorn, Rationale Singularitäten komplexer Flächen Invent. Math. 4 (1967/1968), 336-358.

[4] D. Burns, M. Rapoport, On the Torelli problem for Kählerian K-3 surfaces, Ann. scient. Éc. Norm. Sup. $4^{e}$ ser. 8 (1975), 235-274.

[5] J.H. Conway, N.J.A. Sloane, Sphere packings, lattices and groups, Springer, 1988. 663 pages.

[6] The GAP Group, GAP-Groups, Algorithms and Programming, Version 4.6.5, 2013, http://www.gap-system.org

[7] H. Grauert, Über Modifikationen und exzeptionelle analytische Mengen, Math. Ann. 146 (1962), 331-368.

[8] K. Hashimoto, Finite symplectic actions on the K3 lattice, Preprint 2010, arXiv:1012.2682, 43 pages.

[9] Sh. Kondō, Niemeier lattices, Mathieu groups, and finite groups of symplectic automorphisms of K3 surfaces (with appendix by Sh. Mukai), Duke Math. J. 92, (1998), 593-603. 
[10] Vic. S. Kulikov, Degenerations of $K 3$ surfaces and Enriques surfaces, Izv. Akad. Nauk SSSR Ser. Mat. 41 (1977), no. 5, 1008-1042; English transl. in Math. USSR Izv. 11, (1977) no. 5, 957-989.

[11] Sh. Mukai, Finite groups of automorphisms of K3 surfaces and the Mathieu group, Invent. math. 94, (1988), 183-221.

[12] D. Mumford, The topology of normal singularities of an algebraic surface and a criterion of simplicity, Inst. Hautes Études Sci. Publ. Math. 9 (1961), 5-22.

[13] V.V. Nikulin, On Kummer surfaces, Izv. Akad. Nauk SSSR Ser. Mat. 39 (1975), no. 2, 278-293; English transl. in Math. USSR Izv. 9 (1975), 261-275.

[14] V.V. Nikulin, Finite automorphism groups of Kählerian surfaces of type K3, Uspehi matem. nauk 31 (1976), no. 2, 223-224. (In Russian.)

[15] V.V. Nikulin, Finite automorphism groups of Kähler K3 surfaces, Trudy Mosk. Mat. ob-va V. 38 (1979), 75-137; English transl. in Trans. Moscow Math. Soc. V. 38 (1980), 71-135.

[16] V.V. Nikulin, Integral symmetric bilinear forms and some of their geometric applications, Izv. Akad. Nauk SSSR Ser. Mat. 43 (1979), no. 1, 111-177; English transl. in Math. USSR Izv. 14 (1980), no. 1, 103-167.

[17] V.V. Nikulin, Kahlerian K3 surfaces and Niemeier lattices, Preprint 2013. arXiv:1109.2879v7, 77 pages .

[18] V.V. Nikulin Kählerian K3 surfaces and Niemeier lattices. I Izvestiya RAN: Ser. Mat. 77 (2013), no. 5, 109-154; English translation.: Izvestya: Mathematics 77 (2013), no. 5, 954-997.

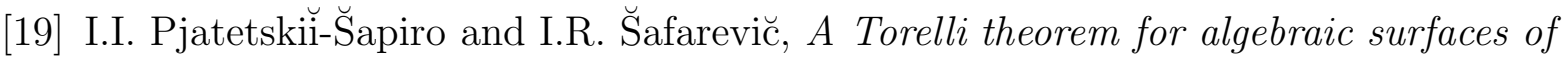
type K3, Izv. AN SSSR. Ser. mat., 35 (1971), no. 3, 530-572; English transl.: Math. USSR Izv. 5 (1971), no. 3, 547-588.

[20] Y. Siu, A simple proof of the surjectivity of the period map of K3 surfaces, Manuscripta Math. 35 (1981), no. 3, 311-321.

[21] A. Todorov, Applications of the Kähler-Einstein-Calabi-Yau metric to moduli of K3 surfaces, Invent. math. 61 (1981), no. 3, 251-265. 
[22] G. Xiao, Galois covers between K3 surfaces, Ann. Inst. Fourier (Grenoble) 46 (1996), 73-88.

\author{
V.V. Nikulin \\ Deptm. of Pure Mathem. The University of Liverpool, Liverpool \\ L69 3BX, UK; \\ Steklov Mathematical Institute, \\ ul. Gubkina 8, Moscow 117966, GSP-1, Russia \\ vnikulin@liv.ac.uk vvnikulin@list.ru \\ Personal page: http://vnikulin.com
}

